
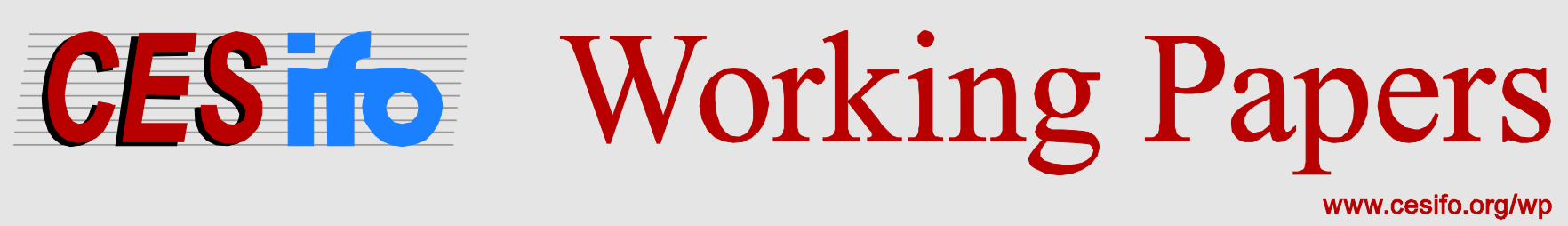

\title{
Overlapping Ownership, R\&D Spillovers, and Antitrust Policy
}

\author{
Ángel L. López \\ Xavier Vives
}

CESIFO WORKING PAPER NO. 5935

CATEGORY 11: INDUSTRIAL ORGANISATION

ORIGINAL VERSION: JUNE 2016

THIS VERSION: JULY 2018

An electronic version of the paper may be downloaded

- from the SSRN website:

- from the RePEc website:

- from the CESifo website: WWW.SSRN.com

Www.RePEc.org

www.CESifo-group.org/wp 


\title{
Overlapping Ownership, R\&D Spillovers, and Antitrust Policy
}

\begin{abstract}
This paper considers cost-reducing R\&D investment with spillovers in a Cournot oligopoly with overlapping ownership. We show that overlapping ownership leads to internalization of rivals. profits by firms and find that, for demand not too convex, increases in overlapping ownership increase (decrease) $R \& D$ and output for high (low) enough spillovers while it increases $R \& D$ but decreases output for intermediate levels of spillovers. There is scope for overlapping ownership to improve welfare provided that spillovers are sufficiently large. The socially optimal degree of overlapping ownership increases with the number of firms, with the elasticity of demand and of the innovation function, and with the extent of spillover effects. In terms of consumer surplus standard, the desirability of overlapping ownership is greatly reduced even under low market concentration. When R\&D has commitment value and spillovers are high the optimal extent of overlapping ownership is higher. The results obtained are robust in the context of a Bertrand oligopoly model with product differentiation.
\end{abstract}

JEL-Codes: D430, L130, O320.

Keywords: competition policy, partial merger, collusion, innovation, minority shareholdings, common ownership, cross-ownership.

Ángel L. López

Departament d'Economia Aplicada

Universitat Autònoma de Barcelona \&

Public-Private Sector Research Center

IESE Business School / Spain

angelluis.lopez@uab.cat

\author{
Xavier Vives \\ IESE Business School \\ University of Navarra \\ Spain - 08034 Barcelona \\ xvives@iese.edu
}

July 2018

We thank Ramon Faulí-Oller for his insightful initial contributions and to the editor and three anonymous referees for very useful suggestions. For helpful comments we thank Isabel Busom, Luis Cabral, Guillermo Caruana, Ricardo Flores-Fillol, Matthew Gentzkow, Richard Gilbert, Gerard Llobet, Peter Neary, Volker Nocke, Patrick Rey, Yossi Spiegel, Konrad Stahl, and Javier Suarez, as well as seminar participants at Boston U., IESE, Mannheim, Bologna, CEMFI, PSE, Rovira i Virgili, Stanford and UCLA as well as from participants at numerous workshops and conferences. Orestis Vravosinos provided excellent research assistance. Financial support from the Spanish Ministry of Economy and Competitiveness under ECO2015-63711-P, for López from AGAUR (under SGR 1326) and for Vives from the European Research Council (Advanced Grant No 789013) and AGAUR (under SGR 1244), is gratefully acknowledged. 


\section{Introduction}

In many industries, overlapping ownership arrangements (OOAs) are prevalent in the form of cross-shareholding agreements among firms or common ownership by investment funds. ${ }^{1}$ The latter in particular has grown tremendously in the last three decades and with investors holding significant stakes in the same industry. The tendency of such OOAs to relax competition has been documented in the airline and banking industries (Azar et al. forthcoming), and it has raised antitrust concerns (Elhauge 2016, Baker 2016). At the same time, there is a debate about whether and why innovative activity and business dynamism have abated recently (e.g., CEA 2016 and Obama's executive order to promote competition) pointing at increased market power as the culprit (e.g., De Loecker and Eeckhout 2017).

The paper contributes by analyzing the interaction of OOAs and R\&D activity in the presence of technological spillovers and deriving testable predictions. OOAs lessen competitive pressure but may have a beneficial effect on investment provided there are positive spillovers across firms. The reason is that OOAs help to internalize the spillover externality, which is especially important for highly innovative industries. ${ }^{2}$ Empirical estimates find that gross social returns to $\mathrm{R} \& \mathrm{D}$ are at least twice as high as the private returns (Bloom et al., 2013). We provide in the paper a welfare analysis that may help elucidate whether the documented increase in OOAs has outrun its social value and derive competition policy implications.

In our benchmark model, firms compete in quantities and invest in cost reduction, and we consider simultaneous output and $\mathrm{R} \& \mathrm{D}$ decisions. That approach aids tractability while helping to capture the imperfect observability of firms' $R \& D$ investment levels. ${ }^{3}$ We consider a general symmetric model of overlapping ownership; this model allows for a range of corporate control structures (as in Salop and O'Brien 2000) and for distinguishing between stock acquisitions made by investors and those made by other firms. The key parameter is the degree of internalization of rivals' profits ( $\lambda$ in our model, ranging from independent ownership, $\lambda=0$, to cartelization $\lambda=1$ ). The parameter $\lambda$ corresponds to what Edgeworth

\footnotetext{
${ }^{1} \mathrm{~A}$ recent example is the car-booking business where apart from cross-ownership (such as Uber and Didi), common investors such as Softbank and Tiger Global hold stakes in Uber, Ola and Grab (see report in the FT by Leslie Hook, September 22, 2017), or such as AFSquare and the mutual fund Fidelity that are also invested in both Uber and Lyft.

${ }^{2}$ Hansen and Lott (1996) explain how shareholder diversification may help internalize externalities.

${ }^{3}$ Even though R\&D investment typically precedes market interaction, this does not mean necessarily that it has strategic commitment value. R\&D investment effort, or even contracts with managers that reward effort, need not be observable. The evidence on the strategic commitment value of R\&D is scant (see Vives 2008).
} 
(1881) termed the coefficient of "effective sympathy" among individuals. Higher degrees of overlapping ownership (common or cross-ownership) lead to a higher $\lambda$. We test the robustness of results by way of a two-stage specification and by considering Bertrand competition with product differentiation. The latter allows to study the impact of market spillovers on the effects of changing $\lambda$. The model subsumes earlier contributions to the literature that were based on linear or constant elasticity of demand and on specific innovation functions. ${ }^{4}$

Our paper seeks to answer the following questions: How do R\&D and output levels vary with the degree of internalization of rivals' profits? How those relationships are affected by structural market parameters (demand and cost conditions, industry technological opportunity, and extent of spillovers)? What are the key determinants of the socially optimal extent of overlapping ownership? How is that optimal level affected by the competition authority's objective (to maximize total or rather consumer surplus)?

The main results on the effects of changes in $\lambda$ can be summarized as follows. If demand is not too convex, then increasing $\lambda$ will increase (resp. decrease) both R\&D and output when spillovers are high (resp. low); for intermediate levels of spillovers, an increase in $\lambda$ will increase R\&D but reduce output. Furthermore, the two thresholds that partition the three regions for spillovers are generally increasing in the level of market concentration, indicating that positive R\&D and output effects of overlapping ownership should be found typically only in markets not too concentrated for given spillover levels.

We identify the degree of market concentration and the extent of spillovers as key determinants of the welfare-optimal degree of internalization $\lambda$ be it according to total surplus (TS) or a consumer surplus (CS) standard. High spillovers increase the desirability of internalizing the profits of rivals. The range of spillovers is typically partitioned into three regions: one optimally with $\lambda=0$ for low levels of spillovers; one optimally (by TS and CS standards) with $\lambda>0$ for high levels of spillovers; and one optimally (by the TS standard only) with $\lambda>0$ in an intermediate region. Furthermore, the optimal interior $\lambda$ (both by TS and CS standards) is increasing in the extent of spillovers. We remark that the CS standard is always more stringent than the TS standard. Numerical results reveal that the (TS-based) socially optimal $\lambda$ is increasing in the number of firms, in the elasticity of demand and of the innovation function (both positively associated to the effectiveness of R\&D), and, indeed, in the level of spillover effects. Qualitatively similar results hold for the CS-based optimal

\footnotetext{
${ }^{4}$ Dasgupta and Stiglitz (1980); Spence (1984); d'Aspremont and Jacquemin (1988); Kamien et al. (1992). Perhaps the work closest to ours in spirit is the paper by Leahy and Neary (1997).
} 
$\lambda$, except that the scope for overlapping ownership is much reduced.

The results provide testable predictions since the sign of the relationship between $R \& D$, output and the degree of overlapping ownership depends on several potentially measurable variables. For example, while an unconditional regression between $R \& D$ and overlapping ownership might not yield significant results, a positive relationship should be found in industries with high enough spillovers, low enough concentration and demand not too convex. In industries with a high effectiveness of $\mathrm{R} \& \mathrm{D}$, the positive association should extend to output. Furthermore, if we check the impact of $\lambda$ on $R \& D$ investment to be negative then we are sure that raising $\lambda$ will decrease consumer welfare. This is so since a positive effect of $\lambda$ on $\mathrm{R} \& \mathrm{D}$ is necessary, but not sufficient, for output, and therefore consumer welfare, to increase with a higher $\lambda$.

The context analyzed here is of more than theoretical interest. The growth of common ownership due to the rise of institutional investors (e.g., by 2010 owning close to $70 \%$ of the US the stock market while in 1950 this was 7-8\%, Blume and Keim 2014) together with the consolidation of the asset management industry (ICI 2017) has been formidable. A consequence is that the proportion of US public firms in the hands of institutional investors which at the same time hold large blocks of other firms in the same industry has grown dramatically (from under $10 \%$ in 1980 to about $60 \%$ in 2010, He and Huang 2017). For example, as reported by Azar et al. (forthcoming), there are substantial common ownership interests of institutional investors (e.g., BlackRock, Vanguard, State Street, Fidelity) in firms in industries as diverse as technology, pharmacies, and banks. ${ }^{5}$ Furthermore, minority shareholdings with cross-ownership patterns are widespread in many industries. ${ }^{6}$

There is growing interest among competition authorities in assessing the competitive effects of partial stock acquisitions due mainly from three factors: (i) the increase in institutional common ownership with investors holding large stakes in firms in the same industry; (ii) the rapid growth of private equity investment firms, which often hold partial ownership interests in competing firms (Wilkinson and White 2007; Nörback et al. 2018); and (iii) some notorious cases, such as Ryanair's acquisition of Aer Lingus's stock. ${ }^{7}$

In the United States, minority shareholdings are examined with reference to merger control rules, the Clayton Act and the Hart-Scott-Rodino Act in particular. Despite that there

\footnotetext{
${ }^{5}$ See also the evidence in Schmalz (2018) who also points out that both passive and active investment strategies contribute to common ownership.

${ }^{6}$ E.g., automobiles, airlines, financial, energy, and steel; see Gilo et al. (2006).

${ }^{7}$ See Gilo (2000) and Brito et al. (forthcoming) for other cases.
} 
is an exception to antitrust scrutiny if the participation is "solely for investment" purposes, OOAs can be challenged if they substantially lessen competition. ${ }^{8}$ Elhauge $(2016,2017)$ proposes to use antitrust to control the effects of rising common ownership; Posner et al. (2016) propose limits to ownership in oligopolistic industries for institutional investors if they want to benefit from a safe harbor from enforcement of the Clayton Act. ${ }^{9}$ In Europe there is debate over the possibly anticompetitive effects of partial ownership. Yet the European Commission (EC) is not authorized to examine the acquisition of minority shareholdings. ${ }^{10}$ In the recent decision in the Dow-Dupont merger EC (2017) states: "the Commission is of the view that (i) a number of large agrochemical companies have a significant level of common shareholding, and that (ii) in the context of innovation competition, such findings provide indications that innovation competition in crop protection should be less intense as compared with an industry with no common shareholding".

The paper proceeds as follows. We review briefly the literature in Section 2. In Section 3, we describe the different types of minority shareholdings that can be analyzed via our model, which is presented in Section 4. That section characterizes the equilibrium responses of output and R\&D to a change in the degree of overlapping ownership. In Section 5, we examine the socially optimal degree of overlapping ownership and then illustrate the results with three leading specifications from the literature: the d'Aspremont-Jacquemin and Kamien-Muller-Zang models, and a constant elasticity model as in Dasgupta and Stiglitz (1980). Section 6 extends our model to allow for strategic R\&D commitments in a twostage game. Section 7 tests the robustness of our results to Bertrand competition with product differentiation. Section 8 explores an alternative interpretation of our model when cooperation in $R \& D$ extends to the product market. We conclude in Section 9. Online appendix A provides details and proofs of our analysis and of the three model specifications considered. Online appendix B develops the analysis of the Bertrand model. We also offer application software (available on the Web), which the reader can use to conduct simulations

\footnotetext{
${ }^{8}$ Section 7 of the Clayton Act prohibits acquisitions (of any part) of a company's stock that "may" substantially lessen competition either by (a) enabling the acquirer to manipulate, directly or indirectly, prices or output or by (b) reducing its own incentives to compete. The substantive passive investor provision states that the prohibition does "not apply to persons purchasing such stock solely for investment and not using the same by voting or otherwise to bring about, or in attempting to bring about, the substantial lessening of competition".

${ }^{9}$ Rock and Rubinfeld (2017) provide a criticism of those views.

${ }^{10} \mathrm{EU}$ Merger Regulation is limited to acquisitions that confer control and therefore is narrower than Section 7 of the Clayton Act. EC (2014) considers how to strengthen merger control and Elhauge (2017) discusses the obstacles in EU law to encompass anti-competitive horizontal shareholdings as well as some avenues for antitrust enforcement. In some European countries (e.g., Austria, Germany, the United Kingdom), national merger control rules give competition authorities the scope to examine minority shareholdings.
} 
with the models.

\section{$2 \quad$ Brief review of the literature}

Previous literature has analyzed the anticompetitive effects of overlapping ownership in Cournot markets (Bresnahan and Salop 1986; Reynolds and Snapp 1986). Farrell and Shapiro (1990) show that silent financial stakes may be welfare increasing in asymmetric oligopolies; here we demonstrate the possibility in a symmetric oligopoly. ${ }^{11}$

Azar et al. (forthcoming) study how common ownership affects market outcomes in the US airline industry, and find that ticket prices are up to $10 \%$ higher on the average route than they would be with no overlapping ownership. Similar results are obtained for the banking industry (Azar et al. 2016). ${ }^{12}$ Gutiérrez and Philippon (2016) examine private fixed investment in the US since the early 2000s, and find underinvestment driven by firms owned by quasi-indexers and belonging to industries which have more concentration and more common ownership. There is some evidence also that common ownership improves efficiency. He and Huang (2017), using data on US public firms from 1980 to 2014, estimate the effect of common ownership on market performance and report that firms increase their market share through common ownership. ${ }^{13}$ The authors note that institutional crossownership facilitates explicit forms of product market collaboration, in particular within industry joint ventures, resource sharing and coordination of R\&D efforts, and improves innovation productivity (in terms of patents per $\$$ spent in $R \& D$ ) as well as operating profitability. ${ }^{14}$ Anton et al. (2017) and Liang (2016) provide evidence of the transmission mechanism of common institutional ownership on managers' incentives finding that relative performance evaluation decreases in industries with more common ownership.

The extant literature (see Gilbert 2006), most of which focuses on the potential benefits

\footnotetext{
${ }^{11}$ Shelegia and Spiegel (2012) study a Bertrand competition model. Gilo et al. (2006) show how minority shareholdings can foster collusion and Heim et al. (2017) find empirical support for the theory.

${ }^{12}$ The work in airlines has been criticized and revisited by Kennedy et al. (2017); in banking by Gramlich and Grundl (2017). Banal et al. (2018) link common ownership measures with markups in a cross-section of US industries. Several authors have found anticompetitive price unilateral effects of cross-ownership arrangements in financial and manufacturing sectors (Dietzenbacher et al. 2000, Brito et al. 2014, and Nain and Wang 2016). See Schmalz (2018) for a survey of the effects of common ownership and their theoretical, empirical and policy underpinnings.

${ }^{13}$ They report also that, among Fama-French US industries, business equipment, healthcare, telecommunications, and energy and finance as well, have high levels of overlapping ownership.

${ }^{14}$ There is evidence also that OOAs offer strategic benefits in product market relationships (Allen and Phillips, 2000; Fee et al. 2006) and in R\&D effort and patent success in the presence of patent complementarities (Geng et al. (2016)). Institutional investors can improve R\&D performance (Bushee 1998, Eng and Shackell 2001, Aghion et al. 2013).
} 
of cooperative R\&D or on how innovation is affected by mergers, has largely ignored the topic of how innovation is affected by minority shareholdings - despite clear evidence that antitrust policy attends closely to innovation. ${ }^{15}$ One of this literature's primary objectives is to examine underprovision of $R \& D$ and the welfare effects of moving from a noncooperative to a cooperative regime in R\&D. For example, Leahy and Neary (1997) show that R\&D cooperation leads to more output, innovation, and welfare when spillovers are positive. We will see that under overlapping ownership, $R \& D$ and output increase only for high enough spillovers. We also identify conditions under which a cartelized Research Joint Venture (RJV) is optimal, generalizing Kamien et al. (1992). Bloom et al. (2013) estimate the extent of spillovers in a panel of US firms from 1981 to 2001 and find that gross social returns to R\&D are at least twice as high as the private returns. Their estimates of technological spillovers obtain a high sensitivity of the stock of knowledge of a firm in relation to the R\&D investment of another firm across a range of industries. They find that technology spillovers are present in all sectors (and are more important than product market spillovers) but with greater importance in high-tech industries such as computers, pharmaceuticals, and telecommunications. Their results imply that the internalization of those technological spillovers is a matter of first-order welfare importance.

\section{Overlapping ownership}

We may consider two types of acquisitions: when investors acquire firms' shares in an industry, called common ownership; and when firms acquire other firms' shares, cross-ownership by firms.

In the first case (common ownership), firms' stakes are held by investors - for example, large institutional investors such as pension or mutual funds, which now have stakes in nearly three fourths of all publicly traded US firms. Consider an industry with $n$ firms and $I \geq n$ investors. Salop and O'Brien (2000) model how the ownership shares and levels of control of investors translate into the objectives of the managers of firms. Each investor derives a total profit from his portfolio holdings. The authors assume that the manager of a firm takes into account shareholders' incentives (through the control weights) and maximizes a weighted average of the shareholders' portfolio profits. We discuss in online appendix A.1.1

\footnotetext{
${ }^{15}$ During the period 2004-2014, 33.6\% of the mergers challenged by the US Department of Justice or the US Federal Trade Commission were characterized as harmful to innovation; of the challenged mergers, $82.5 \%$ were in high-R\&D intensity industries (Gilbert and Greene 2015).
} 
two important cases: silent financial interests (SFI, an ownership interest without influence or control) and proportional control (PC, the firm's manager accounts for shareholders' own-firm interests in other firms in proportion to their respective stakes). ${ }^{16}$ In both cases we assume that each firm has a reference shareholder and each investor acquires a share $\alpha$ of the firms which are not under his control. The reference shareholder keeps an interest $1-(I-1) \alpha$ in his firm and we assume that $\alpha I<1$ so that $1-(I-1) \alpha>\alpha$.

In the second case (cross-ownership, $\mathrm{CO}$ ), we assume that each of the $n$ firms may acquire their rivals' stock in the form of investments with no control rights (e.g., nonvoting shares; see Gilo et al. 2006). This setting features a complex, chain-effect interaction between the profits of firms. Here $\alpha$ denotes a firm's ownership stake in another firm, and the strategy decisions are made by the controlling shareholder.

In each case we show that, when the stakes are symmetric, the firm- $i$ manager's problem is to maximize

$$
\phi_{i}=\pi_{i}+\lambda \sum_{k \neq i} \pi_{k}
$$

where the value of $\lambda$ depends on the type of ownership. Note that $\lambda=0$ corresponds to independently maximizing firms while $\lambda=1$ corresponds to a cartel (or full merger).

In the common ownership cases, the parameter $\lambda$ is the relative weight that the manager of firm $i$ places on the profit of firm $k$ in relation to the own profit (of firm $i$ ) and reflects the control of firm $i$ by investors with financial interests in firms $i$ and $k$. The upper bound of common-ownership is $\alpha=1 / I$, in which case $\lambda=1$ and the managers of firms will maximize total joint profit. We have that for $\alpha<1 / I, \lambda$ is increasing in both $I$ and $\alpha$. The driving force of the comparative statics result is the decline in the interest in the own firm (undiversified stake) of reference investors $1-(I-1) \alpha$ as $I$ or $\alpha$ increase. ${ }^{17}$

In the cross-ownership case $\lambda$ is the ratio of the stake of firm $i$ in firm $k$ over the claims of firm $i$ on its own firm and on firm $k$. It follows that the upper bound of cross-ownership is $\alpha=1 /(n-1)$, in which case $\lambda$ tends to 1 as $\alpha$ approaches $1 /(n-1)$. We have that $\lambda$ is increasing in $n$ and $\alpha .^{18}$

\footnotetext{
${ }^{16}$ Other governance structures are discussed in Salop and O'Brien (2000). Any structure that preserves symmetry will be encompassed by our approach. Banal-Estanol et al. (2018) extend the model to allow for a partition of active and passive investors, which preserves symmetry in the $\lambda$ 's, with the later having less control than their stake in firms.

${ }^{17}$ The mechanism can be grasped more directly in a simpler ownership structure with proportional control. If we had $I$ investors in each firm with a total interest $1-\alpha$ and a common investor with stake $\alpha$ in all firms, then $\lambda^{\mathrm{PC}}=\alpha^{2} /\left[(1-\alpha)^{2} I^{-1}+\alpha^{2}\right]$. As $I \rightarrow \infty$, undiversified investors become small, and $\lambda^{\mathrm{PC}} \rightarrow 1$; while if each firm has a large reference investor $\left(I=1\right.$ with $1-\alpha$ large), then $\lambda^{\mathrm{PC}}$ will be small.

${ }^{18}$ This is so, since for given $\alpha$, an additional firm reduces the share of profits that firm $j$ receives from
} 
Table 1 summarizes the value of $\lambda$ according to the type of overlapping ownership (SFI, $\mathrm{PC}$, or $\mathrm{CO})$. We can see that more investors and higher investment stakes are both positively associated with $\lambda$. In addition, it is straightforward to show that $\lambda^{\mathrm{PC}}>\lambda^{\mathrm{SFI}}$ and that for $I=n, \lambda^{\mathrm{SFI}}>\lambda^{\mathrm{CO}}$. The implication is that, in order to attain the same degree of profit internalization (and for a given number of firms $n$ ), the investment stake with proportional control must be lower than with silent financial interests, $\alpha^{\mathrm{PC}}<\alpha^{\mathrm{SFI}}$, which in turn must be lower than with cross-ownership by firms, $\alpha^{\mathrm{SFI}}<\alpha^{\mathrm{CO}}$, for $I=n .{ }^{19}$ Consistently with the results found here, Anton et al. (2017) show that in industries with higher degrees of common ownership (i.e., higher $\alpha$ ), relative performance evaluation is used less to provide incentives to managers, which means that the degree of profit internalization $\lambda$ is higher.

Table 1: Profit Internalization $(\lambda)$ under Different Ownership Structures

\begin{tabular}{c|c||c||c}
\hline & $\begin{array}{c}\text { Common Ownership, } \\
\text { Silent Financial Interests }\end{array}$ & $\begin{array}{c}\text { Common Ownership, } \\
\text { Proportional Control }\end{array}$ & $\begin{array}{c}\text { Cross-ownership } \\
\text { (by firms) }\end{array}$ \\
\hline$\lambda$ & $\frac{\alpha}{1-(I-1) \alpha}$ & $\frac{2 \alpha[1-(I-1) \alpha]+(I-2) \alpha^{2}}{[1-(I-1) \alpha]^{2}+(I-1) \alpha^{2}}$ & $\frac{\alpha}{1-(n-2) \alpha}$ \\
\hline
\end{tabular}

\section{Framework and equilibrium}

We consider an industry consisting of $n \geq 2$ identical firms, where each firm $i=1, \ldots, n$ chooses simultaneously their R\&D level $\left(x_{i}\right)$ and production quantity $\left(q_{i}\right)$. Firms produce a homogeneous good characterized by a smooth inverse demand function $f(Q)$, where $Q=$ $\sum_{i} q_{i}$. We make the following three assumptions.

A.1. $f(Q)$ is twice continuously differentiable, where (i) $f^{\prime}(Q)<0$ for all $Q \geq 0$ such that

its own operational profit in relation to the received operational profit from any other firm $k$ (in proportion $\alpha$ ). Similarly, for given $n$, a higher $\alpha$ increases the share of profits that firm $j$ receives from the operational profit of firm $k$, while it reduces the share of profits that firm $j$ receives from its own operational profit, thereby also increasing $\lambda$.

${ }^{19}$ The intuition for $\alpha^{\mathrm{SFI}}<\alpha^{\mathrm{CO}}$ is easy to grasp for $n=2$. Then under SFI the manager of $i$ puts weight $1-\alpha$ in the own firm's profits $\pi_{i}$ while under CO the manager of $i$ puts weight 1 on those profits (since he is maximizing $\left.\phi_{i}=\left(\pi_{i}+\alpha \pi_{k}\right) /\left(1-\alpha^{2}\right)\right)$; note that $\alpha^{2} \phi_{i}$ is the share of the total profit of $j$ that firm $i$ recovers through its silent investment $\alpha$ in firm $k$ (the chain effect). And consequently, $1-\alpha^{2}$ is the share of the total profit of $i$ net of the chain effect. In both cases the manager of $i$ puts weight $\alpha$ in the profits of the other firm. 
$f(Q)>0$ and (ii) the elasticity of the slope of the inverse demand function,

$$
\delta(Q) \equiv \frac{Q f^{\prime \prime}(Q)}{f^{\prime}(Q)}
$$

is constant and equal to $\delta$.

The parameter $\delta$ is the curvature (relative degree of concavity) of the inverse demand function, so demand is concave for $\delta>0$ and is convex for $\delta<0$. Furthermore, demand is log-concave for $1+\delta>0$ and is log-convex for $1+\delta<0$. If $1+\delta=0$, then demand is both log-concave and log-convex. ${ }^{20}$ The family of inverse demand functions for which $\delta(Q)$ is constant, includes linear or constantly elastic cases, and can be represented as

$$
f(Q)= \begin{cases}a-b Q^{\delta+1} & \text { if } \delta \neq-1 \\ a-b \log Q & \text { if } \delta=-1\end{cases}
$$

here $a$ is a nonnegative constant and $b>0$ (resp., $b<0)$ if $\delta \geq-1$ (resp., $\delta<-1$ ).

A.2. The marginal production cost or innovation function of firm $i$, or $c_{i}$, is independent of output and is decreasing in both own and rivals' R\&D as follows: $c_{i}=c\left(x_{i}+\beta \sum_{j \neq i} x_{j}\right)>0$, where $c^{\prime}<0, c^{\prime \prime} \geq 0$, and $0 \leq \beta \leq 1$ for $i \neq j$.

A.3. The cost of R\&D level $x_{i}$ is given by $\Gamma\left(x_{i}\right)$, where $\Gamma(0)=0, \Gamma^{\prime}>0$, and $\Gamma^{\prime \prime} \geq 0$.

The parameter $\beta$ represents the spillover level of the R\&D activity. Since we focus on symmetric firms, we assume symmetric spillover levels; moreover, R\&D outcomes are imperfectly appropriable to an extent that varies between 0 and 1 . The intensity of spillover levels is quite heterogeneous across industries. Bloom et al. (2013) find an average sensitivity of .4 to .5 of the stock of knowledge of a firm in relation to the R\&D investment of another firm. However, the dispersion of the estimates across industries is large.

Firm $i$ 's profit is given by

$$
\pi_{i}=f(Q) q_{i}-c\left(x_{i}+\beta \sum_{j \neq i} x_{j}\right) q_{i}-\Gamma\left(x_{i}\right),
$$

\footnotetext{
${ }^{20}$ This class of demands features a constant pass-through from cost to price of $(2+\delta)^{-1}$ for a monopoly firm (Bulow and Pfleiderer 1983). We note that $\delta$ is also related to the marginal consumer surplus from increasing output - that is, to $\mathrm{MS}=-f^{\prime}(Q) Q$. Weyl and Fabinger (2013) argue that $\left.\epsilon_{\mathrm{MS}} \equiv \mathrm{MS} /\left(\mathrm{MS}^{\prime} Q\right)\right)$ measures the curvature of the logarithm of demand. Under A.1, we can write $1 / \epsilon_{\mathrm{MS}}=1+\delta$.
} 
and the objective function for the manager of firm $i$ is to maximize $\phi_{i}=\pi_{i}+\lambda \sum_{k \neq i} \pi_{k}$ choosing $\left(q_{i}, x_{i}\right)$. The model represents distinct scenarios depending on the values of $\beta$ and $\lambda$. When $\lambda \in(0,1)$ and $\beta \in[0,1)$, firms compete in the presence of partial ownership interests and the $R \& D$ outcomes are imperfectly appropriable. When $\lambda \in(0,1)$ and $\beta=1$, firms form a Research Joint Venture (RJV) under which all R\&D outcomes are fully shared among RJV members and the duplication of $\mathrm{R} \& \mathrm{D}$ efforts is avoided. When $\lambda=\beta=1$, firms form a "cartelized" RJV. ${ }^{21}$ If $\lambda=0$ then there is no overlapping ownership.

For markets with cross-shareholdings, a modified HHI is proposed by Bresnahan and Salop (1986). This index corresponds to the market share-weighted Lerner index in a Cournot market, and we write MHHI $=\left(\sum_{i} s_{i} L_{i}\right) \eta$. Here $s_{i}$ and $L_{i}$ are (respectively) the market share and Lerner index of firm $i$; the term $\eta$ denotes the demand (price) elasticity. ${ }^{22}$ In our case it is easy to see that, for a given common marginal cost, $(p-c) / p=\mathrm{MHHI} / \eta$ at a symmetric Cournot equilibrium; here MHHI $=\Lambda / n$ for $\Lambda=1+\lambda(n-1)$, which is monotone in $\lambda$. When $\lambda=0$ we have the standard HHI for a symmetric solution, $1 / n$, and if $\lambda=1$ then the modified HHI is equal to 1.

Now we consider symmetric solutions of the game. Let $B \equiv 1+\beta(n-1)$; then $B x$ is the "effective" investment that lowers costs for a firm. Let $\tau \equiv 1+\lambda \beta(n-1)$. Then $-c^{\prime}(B x) q \tau$ is the marginal effect of investment by a firm on its internalized profit $\phi_{i}$. A symmetric interior equilibrium $\left(Q^{*}=n q^{*}, x^{*}\right)$ must solve the first-order necessary conditions for the maximization of $\phi_{i}\left(\partial \phi_{i} / \partial q_{i}=0, \partial \phi_{i} / \partial x_{i}=0\right)$ :

$$
\begin{gathered}
\frac{f\left(Q^{*}\right)-c\left(B x^{*}\right)}{f\left(Q^{*}\right)}=\frac{\mathrm{MHHI}}{\eta\left(Q^{*}\right)} \\
-c^{\prime}\left(B x^{*}\right) \frac{Q^{*} \tau}{n}=\Gamma^{\prime}\left(x^{*}\right) .
\end{gathered}
$$

Here $\eta\left(Q^{*}\right)=-f\left(Q^{*}\right) /\left(Q^{*} f^{\prime}\left(Q^{*}\right)\right)$ is the elasticity of demand. Equation (2) is the modified Cournot-Lerner pricing formula; expression (3) equates the marginal benefit and marginal cost of investment by a firm taking into account its internalized profit $\phi_{i}$. Note that both MHHI and $\tau$ are increasing in $\lambda$ and therefore respectively exert pressure to reduce output

\footnotetext{
${ }^{21}$ We follow here the terminology in Kamien et al. (1992). d'Aspremont and Jacquemin (1988) identify cooperation in R\&D only, in our terminology, with $\lambda=0$ for output decisions and $\lambda=1$ for R\&D decisions with $\beta \in[0,1]$. This situation is termed an "R\&D cartel" by Kamien et al. (1992). For the latter the situation where $\beta=1$ and $\lambda=1$ only for $\mathrm{R} \& \mathrm{D}$ decisions is termed "R\&D cooperation".

${ }^{22}$ Azar et al. (forthcoming) use the MHHI (in terms of control and share rights) to measure anticompetitive incentives stemming from financial interests in the US airline industry. These authors find that, in year 2013, the increased market concentration generated by such financial interests was more than 10 times greater than the HHI increase above which mergers are likely to generate antitrust concerns.
} 
(or increase prices and margins) and to increase investment.

Let second-order derivatives be denoted, at symmetric solutions, by $\partial_{z_{i} z_{j}} \phi_{i} \equiv \partial^{2} \phi_{i} / \partial z_{i} \partial z_{j}$ and $\partial_{h z_{i}} \phi_{i} \equiv \partial^{2} \phi_{i} / \partial h \partial z_{i}$ (with $h=\beta, \lambda$, and $z=q, x$ ). We assume that the following regularity conditions hold:

$$
\Delta_{q} \equiv \partial_{q_{i} q_{i}} \phi_{i}+(n-1) \partial_{q_{i} q_{j}} \phi_{i}<0 ; \Delta_{x} \equiv \partial_{x_{i} x_{i}} \phi_{i}+(n-1) \partial_{x_{i} x_{j}} \phi_{i}<0,
$$

and

$$
\Delta \equiv \Delta_{q} \Delta_{x}-\left(\partial_{x_{i} q_{i}} \phi_{i}\right)^{2} \tau B>0
$$

Together these conditions imply that (2) and (3) both have a unique solution if they hold globally. ${ }^{23}$ Condition $\Delta_{q}<0$ is a standard stability condition in a quantity Cournot game (e.g., Dixit (1986)) and implies that $\partial_{q_{i} q_{i}} \phi_{i}<0$. Condition $\Delta_{x}=-c^{\prime \prime}\left(B x^{*}\right) q^{*} \tau B-\Gamma^{\prime \prime}\left(x^{*}\right)<0$ is the equivalent for the innovation choice (e.g., Leahy and Neary (1997), Vives (2008)). It is noteworthy that $\Delta_{x}<0$ requires that at least one of $c^{\prime \prime}$ and $\Gamma^{\prime \prime}$ be positive and implies that $\partial_{x_{i} x_{i}} \phi_{i}<0$. (See Table 4 in the Appendix.)

If $\Delta\left(Q^{*}, x^{*}\right)>0$ then we say that the equilibrium is regular. In particular, we assume that there is a unique regular symmetric interior equilibrium $\left(Q^{*}, x^{*}\right) \cdot{ }^{24}$ The focus of our paper is on characterizing that equilibrium.

\subsection{Model specification examples}

We will consider the well-known R\&D model specifications - with linear (and therefore logconcave) demand - of d'Aspremont-Jacquemin (AJ) and Kamien-Muller-Zang (KMZ); we also consider a constant elasticity (CE) model with log-convex demand that is similar to the Dasgupta and Stiglitz (1980) model but with spillover effects. In AJ $c(\cdot)$ is linear and $\Gamma(\cdot)$ is quadratic while in $\mathrm{KMZ}$ and $\mathrm{CE}, c(\cdot)$ is strictly convex and $\Gamma(\cdot)$ linear. The AJ and the KMZ model specifications are only equivalent for a subset of spillover values (which includes the case of no spillovers and depends on the number of firms). ${ }^{25}$ Table 2 summarizes these model

\footnotetext{
${ }^{23}$ This is so since they imply that the Jacobian of the FOC at the symmetric solution is negative definite. We have then that the Gale-Nikaido univalence conditions are fulfilled (see Section 2.5 in Vives 1999).

${ }^{24}$ Provided $\phi_{i}$ is strictly concave in $\left(q_{i}, x_{i}\right)$ and some mild boundary conditions hold, then an interior equilibrium will exist. (Strict concavity of $\phi_{i}$ is ensured with the usual differential second-order conditions, see A.1.2 in the online appendix.)

${ }^{25}$ Furthermore, while in AJ the joint returns to scale (in R\&D expenditure and number of firms) are decreasing, constant, or increasing when $\beta$ is less than, equal to, or greater than $1 /(n+1)$; in KMZ the joint returns to scale are always nonincreasing if $\beta \leq 1$ (Proposition 4.1 in Amir 2000). See also Section A.2 of the online appendix.
} 
Table 2: Model Specifications

\begin{tabular}{cc||c||c}
\hline & AJ & KMZ & CE \\
\hline Demand & $f(Q)=a-b Q$ & $f(Q)=a-b Q$ & $f(Q)=\sigma Q^{-\varepsilon}, 0<\varepsilon<1$ \\
& $\delta=0 ; a, b>0$ & $\delta=0 ; a, b>0$ & $\delta=-(1+\varepsilon) ; a=0, b=-\sigma<0$ \\
$c(\cdot)$ & $\bar{c}-x_{i}-\beta \sum_{j \neq i} x_{j}$ & $\bar{c}-\left[(2 / \gamma)\left(x_{i}+\beta \sum_{j \neq i} x_{j}\right)\right]^{1 / 2}$ & $\kappa\left(x_{i}+\beta \sum_{j \neq i} x_{j}\right)^{-\alpha} ; \alpha, \kappa>0$ \\
$\Gamma(x)$ & $(\gamma / 2) x^{2}$ & $x$ & $x$ \\
\hline
\end{tabular}

specifications (where $\delta$ is the demand curvature), and tables A1 and A2 (in online appendix A.2.1) provide, respectively, equilibrium values of output and $R \& D$ that are obtained by solving equations (2) and (3), and the sufficient second-order and regularity conditions for each specification. In all cases outputs are strategic substitutes since $\delta>-2$.

\subsection{Comparative statics with respect to $\lambda$}

We note first that if an increase in the degree of internalization of rivals' profits $(\lambda)$ lowers $\mathrm{R} \& \mathrm{D}$ then it must lower output also (but the converse is not true). This is so because a lower R\&D leads to higher marginal cost and a higher $\lambda$ relaxes competition. This leaves three possibilities. If $\lambda$ increases then either both output and R\&D fall or rise, or output falls and R\&D rises. A higher $\lambda$ tends to decrease incentives to produce, because of its anti-competitive effect, but in the presence of spillovers raises incentives to invest in $R \& D$ reducing cost, and has an output expansion effect, because it internalizes the externality of independent $\mathrm{R} \& \mathrm{D}$ choices. The question is how the output and investment decisions interact.

We are interested in how output and $\mathrm{R} \& \mathrm{D}$ respond, in equilibrium, to a change in $\lambda$. The sign of the derivatives $\partial q^{*} / \partial \lambda$ and $\partial x^{*} / \partial \lambda$ can be ambiguous. Differentiating totally the FOCs, we obtain

$$
\begin{aligned}
& \partial q^{*} / \partial \lambda=\left[\left(\partial_{\lambda x_{i}} \phi_{i}\right)\left(\partial_{x_{i} q_{i}} \phi_{i}\right) B-\left(\partial_{\lambda q_{i}} \phi_{i}\right) \Delta_{x}\right] / \Delta \\
& \partial x^{*} / \partial \lambda=\left[\left(\partial_{\lambda q_{i}} \phi_{i}\right)\left(\partial_{x_{i} q_{i}} \phi_{i}\right) \tau-\left(\partial_{\lambda x_{i}} \phi_{i}\right) \Delta_{q}\right] / \Delta .
\end{aligned}
$$

For a given $x$, the extent of overlapping ownership $\lambda$ has a negative effect on output: $\partial_{\lambda q_{i}} \phi_{i}=f^{\prime}(Q) q(n-1)<0$. This is the well-known effect of reducing output so as to increase price when the profit of rivals is being taken into account. For a given $q$, however, $\lambda$ has a positive effect on investment: $\partial_{\lambda x_{i}} \phi_{i}=-\beta q(n-1) c^{\prime}(x B)>0$. This is the internalizing 
effect of spillovers with a higher $\lambda$, and its strength depends directly on the size $(\beta)$ of those spillovers. The total impact of $\lambda$ on the equilibrium values of per-firm output and R\&D will depend on which of the two previous effects dominates. What is clear is that, if $\partial x^{*} / \partial \lambda \leq 0$, then $\partial q^{*} / \partial \lambda<0$ because $\partial_{x_{i} q_{i}} \phi_{i}=-c^{\prime}(x B)>0$ (output and R\&D are complements for a firm). That is, an increase in R\&D investment is necessary (but not sufficient) for output to rise with increasing $\lambda$. When $\beta$ is small, the positive effect on investment is small and so the negative effect on output dominates. Then $q^{*}$ decreases with $\lambda$ and, as a result, firms invest less also when $\lambda$ increases - given that the benefit to firms from investing in R\&D decreases proportionally with output.

We shall use $R_{\mathrm{I}}$ to denote the region in which $\partial q^{*} / \partial \lambda<0$ and $\partial x^{*} / \partial \lambda \leq 0$. If $\beta$ is sufficiently high, then the positive effect on $R \& D$ reduces significantly the unit cost of production, which in turn stimulates output. Two effects are present in this case. On the one hand, firms want to reduce output in order to increase competitors' profit and hence their own financial profit. On the other hand, firms now have incentives to produce more because they are more efficient. If the first effect dominates, then $\partial q^{*} / \partial \lambda<0$ and $\partial x^{*} / \partial \lambda>0$ (we label this region $\left.R_{\mathrm{II}}\right)$. But if the second effect dominates, then $\partial q^{*} / \partial \lambda>0$ and $\partial x^{*} / \partial \lambda>0$ (region $R_{\mathrm{III}}$ ). Which of these two cases arises in equilibrium will depend on the extent of the spillovers. We find that, whereas $R_{\mathrm{I}}$ always exists, regions $R_{\mathrm{II}}$ and $R_{\mathrm{III}}$ might not exist.

We next derive the conditions and threshold values (in terms of $\beta$ ) that define the boundaries of the regions characterizing the signs of $\partial x^{*} / \partial \lambda$ (Lemma 1) and $\partial q^{*} / \partial \lambda\left(\right.$ Lemma 2). ${ }^{26}$

LEMMA 1 At equilibrium, $\operatorname{sign}\left\{\partial x^{*} / \partial \lambda\right\}=\operatorname{sign}\{\beta(1+n+\delta \Lambda)-1\}$.

COROLLARY 1 For any fixed $\lambda$ and for any $\beta \in[0,1]$, only $R_{\mathrm{I}}$ exists (with $\partial x^{*} / \partial \lambda \leq 0$ ) if and only if demand is convex enough - that is, iff $\delta \leq-n / \Lambda .{ }^{27}$ This statement holds for any $\lambda$ in $[0,1]$ provided that $\delta \leq-n$.

We can interpret the critical spillover threshold for $\beta$ in terms of the cost pass-through coefficient (i.e., the rate at which the price changes with marginal cost). This threshold is equal to the industry-wide per-firm cost pass-through coefficient $\left(P^{\prime}(c) / n\right)$ multiplied by the internalized cost-reducing effect of a unit increase in R\&D expenditures by each firm $(\tau)$;

\footnotetext{
${ }^{26}$ The effects on output and investment of changes in $\lambda$ do not depend on the assumption of a constant $\delta$. However, the characterization of the boundary in $\beta$ space between $R_{\mathrm{I}}$ and $R_{\mathrm{II}}$ is made much simpler with $\delta$ constant.

${ }^{27}$ When $\delta>-(n+1) / \Lambda$, there exists a positive threshold of spillover above which $\partial x^{*} / \partial \lambda>0$; however, that threshold exceeds unity unless $\delta>-n / \Lambda$.
} 
formally, we have $\operatorname{sign}\left\{\partial x^{*} / \partial \lambda\right\}=\operatorname{sign}\left\{\beta-P^{\prime}(c) \tau / n\right\}$. Firms, in principle, should be less interested in reducing costs when doing so translates, in effect, into lower prices. Note that $P^{\prime}(c)$ is increasing with the degree of convexity of the demand..$^{28}$

A consequence of Lemma 1 is that the threshold for spillovers to induce $\partial x^{*} / \partial \lambda \leq 0$ is decreasing (resp. increasing) in $\lambda$ when demand is concave (resp. convex) - that is, when $\delta>0(\operatorname{resp} . \delta<0) \cdot{ }^{29}$

If demand is extremely convex, then increases in overlapping ownership are so restrictive of output that they induce $\partial x^{*} / \partial \lambda<0$, in which case only $R_{\mathrm{I}}$ exists for any $\beta$. And since $\mathrm{MHHI}=\Lambda / n$, the applicable condition is that $\delta \leq-(\mathrm{MHHI})^{-1}$. Corollary 1 implies that the degree of demand convexity required for only $R_{\mathrm{I}}$ to exist is decreasing in the concentration measured by MHHI; in other words, the condition is less restrictive in markets that are more concentrated. The corollary implies also that $R_{\mathrm{II}}$ can exist only when quantities are strategic substitutes. ${ }^{30}$ Indeed, if quantities are instead strategic complements (i.e., if $\partial_{q_{i} q_{j}} \phi_{i}>0$, which holds when $\delta<-n(1+\lambda) / \Lambda$, then the condition $\delta<-n / \Lambda$ always holds and only $R_{\mathrm{I}}$ exists. When $\delta$ is such that $-n(1+\lambda) / \Lambda<\delta<-n / \Lambda$, quantities are strategic substitutes (as e.g. when demand is log-concave) but again only $R_{\mathrm{I}}$ exists. If $\delta>-n / \Lambda$, then quantities are strategic substitutes and $R_{\mathrm{II}}$ exists (see Figure 5 in online appendix A.1.2 which depicts the existence of regions $R_{\mathrm{I}}$ and $R_{\mathrm{II}}$ in $(\lambda, \delta)$ space together with conditions for outputs to be strategic substitutes or complements). ${ }^{31}$

As regards the comparative statics on output, totally differentiating the first-order condition (FOC) with respect to $\lambda$ yields

$$
\operatorname{sign}\left\{\partial q^{*} / \partial \lambda\right\}=\operatorname{sign}\left\{\partial_{\lambda q_{i}} \phi_{i}+B\left(\partial_{x_{i} q_{i}} \phi_{i}\right) \partial x^{*} / \partial \lambda\right\}
$$

here $B=1+\beta(n-1)$ captures the effect, on each firm's marginal cost, of a unit increase in R\&D by all firms. At equilibrium, the impact on output of a higher degree of overlapping

\footnotetext{
${ }^{28}$ Let $P(c) \equiv f\left(n q^{*}(c)\right)$; then $P^{\prime}(c)=f^{\prime}\left(n q^{*}\right) n\left(d q^{*} / d c\right)=n /[\Lambda(1+\delta)+n]$. Since the stability condition $\Delta_{q}<0$ holds when $\Lambda(1+\delta)+n>0$, it follows that $P^{\prime}(c)>0$. Furthermore, the pass-through increases with the number of firms when demand is log-concave $(\delta>-1)$. See Weyl and Fabinger (2013).

${ }^{29}$ So for $\delta>0$, if $\partial x^{*} / \partial \lambda>0$ for some $\lambda$ then that inequality must hold also for larger values of $\lambda$. Analogously: for $\delta<0$, if $\partial x^{*} / \partial \lambda<0$ for some $\lambda$ then that inequality holds also for larger values of $\lambda$.

${ }^{30}$ This is so when $\delta>-(1+\lambda) n / \Lambda$ (see Table 4 in the Appendix), which holds for all $\lambda$ and $n$ when $\delta>-2$ - in other words, the convexity of inverse demand must not be too high, which in turn implies that marginal revenue is strictly decreasing in output. It is worth noting that, in order for strict concavity of $\phi_{i}$ with respect to $q_{i}\left(\partial_{q_{i} q_{i}} \phi_{i}<0\right)$ at a symmetric equilibrium to be guaranteed for all $\lambda$, we need the condition $\delta>-2$ (which guarantees strategic substitutability for all $\lambda$ and $n$ ). The concavity condition is $\delta>-2 n / \Lambda$, and it is the strictest for $\lambda=1$ (in which case it reduces to $\delta>-2$ ).

${ }^{31} \mathrm{It}$ is worth noting that cost reduction efforts are strategic substitutes $\left(\partial_{x_{i} x_{j}} \phi_{i}<0\right)$ provided that $\beta>0$ (see Table 4 in the Appendix).
} 
ownership depends directly on its effect on marginal profit with respect to output $\left(\partial_{\lambda q_{i}} \phi_{i}\right)$ and indirectly through its effect on the $\mathrm{R} \& \mathrm{D}$ effort of each firm at equilibrium. Recall that, since $\partial_{x_{i} q_{i}} \phi_{i}>0$, it follows that if $\partial x^{*} / \partial \lambda \leq 0$ then $\partial q^{*} / \partial \lambda<0\left(R_{\mathrm{I}}\right)$. By Lemma 1 we know that, if spillovers are sufficiently high and demand is not too convex, then $\partial x^{*} / \partial \lambda>0$; however, the sign of $\partial q^{*} / \partial \lambda$ can be negative $\left(R_{\mathrm{II}}\right)$ or positive $\left(R_{\mathrm{III}}\right)$.

We derive an inverse measure of $\mathrm{R} \& \mathrm{D}$ effectiveness in terms of the model's basic elasticities. This measure $H$ is an indirect function of $\beta$, since the equilibrium depends on $\beta$, and provides the appropriate threshold for the positive effect of minority shareholdings on $R \& D$ investments to dominate its negative effect on output. Let $\chi\left(B x^{*}\right) \equiv-c^{\prime \prime}\left(B x^{*}\right) B x^{*} / c^{\prime}\left(B x^{*}\right) \geq$ 0 be the elasticity of the slope of the innovation function (i.e., the relative convexity of $c(\cdot)$ ) evaluated at the effective R\&D, $B x^{*}$; and let $y\left(x^{*}\right) \equiv \Gamma^{\prime \prime}\left(x^{*}\right) x^{*} / \Gamma^{\prime}\left(x^{*}\right) \geq 0$ be the elasticity of the slope of the investment cost function. Our regularity assumptions imply that either $c^{\prime \prime}>0$ or $\Gamma^{\prime \prime}>0$ (or both). If $\Gamma^{\prime \prime}\left(x^{*}\right)>0$, let $\xi\left(Q^{*}, x^{*}\right) \equiv-\left(c^{\prime}\left(B x^{*}\right)\right)^{2} /\left(f^{\prime}\left(Q^{*}\right) \Gamma^{\prime \prime}\left(x^{*}\right)\right)>0$ measure the relative effectiveness of $R \& D .{ }^{32}$ Note also that a higher ratio $y / \chi$ means that the investment is more effective in reducing costs. Then $H$ can be written as

$$
H=\frac{1}{\xi\left(Q^{*}, x^{*}\right)}\left(1+\frac{\chi\left(B x^{*}\right)}{y\left(x^{*}\right)}\right),
$$

evaluated at the equilibrium $\left(Q^{*}, x^{*}\right)$. Note that $H$ is positive and decreasing in the effectiveness of R\&D as measured by $\xi$ and by $y / \chi$.

LEMMA 2 Let $B=1+\beta(n-1)$. At equilibrium, $\operatorname{sign}\left\{\partial q^{*} / \partial \lambda\right\}=\operatorname{sign}\{\beta B-H\}$.

For $\beta>0$ we have that the term $H / \beta$ provides the appropriate threshold for $B$ (the effect on each firm's marginal cost of a unit increase in R\&D by all firms) for a rise in $\lambda$ to increase output. Therefore, if $B>H / \beta$ then the positive effect of overlapping ownership on $\mathrm{R} \& \mathrm{D}$ investments dominates its negative effect on output. The values of $H$ for each model specification are presented in Table $3 .^{33}$ Note that $H$ is independent of $\lambda$ under the AJ and KMZ models but is strictly increasing in $\lambda$ under the CE model. As we shall discuss later, the relationship between $H$ and $\lambda$ has important consequences for the optimal welfare policy. It is worth noting that the effectiveness of R\&D increases with the elasticity of demand $\left(b^{-1}\right.$; $\left.\varepsilon^{-1}\right)$ and with the elasticity of the innovation function $\left(\gamma^{-1} ; \alpha\right)$ in the specified models.

\footnotetext{
${ }^{32}$ As defined by Leahy and Neary (1997, Sec. V, p. 654).

${ }^{33} \mathrm{In} \mathrm{AJ}, y=1$ and $\chi=0$; in KMZ, $y=0$ and $\chi=1 / 2$; in CE, $y=0$ and $\chi=\alpha+1$.
} 
Table 3: $H$ (Inverse Measure of R\&D Effectiveness)

\begin{tabular}{cc||c||c}
\hline & AJ & KMZ & CE \\
\hline$H$ & $b \gamma$ & $b \gamma B$ & $B\left(\frac{\alpha+1}{\alpha}\right) \frac{\varepsilon}{n-\varepsilon \Lambda} \tau$ \\
\hline
\end{tabular}

We introduce the following mild assumption on $H:[0,1] \rightarrow \mathbb{R}^{+}$(considered as a function of $\beta$ ). $H$ is continuous (see proof of Lemma 2).

A.4. $H(\beta) / \beta$ is downward sloping.

Under assumption A.4, the equation $B=H(\beta) / \beta$ has at most a unique positive solution ( since $\lim _{\beta \rightarrow 0} H(\beta) / \beta=\infty$ ). This assumption is sufficient but not necessary for uniqueness. An (almost) necessary and sufficient condition for uniqueness is that $H(\beta) / \beta B$ is decreasing in $\beta$ whenever $B=H(\beta) / \beta$. Denote that solution by $\beta^{\prime}$; then, for $\beta>\beta^{\prime}$ we have that $\partial q^{*} / \partial \lambda>0$. Assumption A.4 seems not to be restrictive in light of the model specifications typically used in the literature; it is fulfilled in AJ and $\mathrm{KMZ}$. In $\mathrm{CE}, H(\beta) / \beta B$ is strictly decreasing in $\beta$. Assumption A.4 does not guarantee that there exists $\beta^{\prime}<1$, so $R_{\text {III }}$ may fail to exist. We have that a solution $\beta^{\prime}<1$ exists if $n>H(1)$. Our next corollary states the results formally.

COROLLARY 2 Under $A .4$, if $n>H(1)$ then region $R_{\mathrm{III}}$ exists when $\beta>\beta^{\prime}$ with $\beta^{\prime}<1$ (where $\beta^{\prime}$ is the unique positive solution to $\beta B-H(\beta)=0$ ).

Using Lemmata 1 and 2 - and observing that $\delta>-n / \Lambda$ implies that $1+n+\delta \Lambda>0$-we obtain the following result.

PROPOSITION 1 Let $\Lambda=1+\lambda(n-1)$. Under assumptions $A .1-A .3$, if demand is sufficiently convex $(\delta \leq-n / \Lambda)$ then only region $R_{\mathrm{I}}$ exists. Otherwise, assume A.1-A.4, $n>H(1)$, and let $\beta(\lambda)=1 /(1+n+\delta \Lambda)$ and $\beta^{\prime}(\lambda)$ be as defined in Corollary 2. Then the following statements hold:

(i) if $\beta \leq \underline{\beta}(\lambda)$, then $\partial q^{*} / \partial \lambda<0$ and $\partial x^{*} / \partial \lambda \leq 0\left(R_{\mathrm{I}}\right)$;

(ii) if $\beta(\lambda)<\beta \leq \beta^{\prime}(\lambda)$, then $\partial q^{*} / \partial \lambda \leq 0$ and $\partial x^{*} / \partial \lambda>0\left(R_{\mathrm{II}}\right)$;

(iii) if $\beta>\beta^{\prime}(\lambda)$, then $\partial q^{*} / \partial \lambda>0$ and $\partial x^{*} / \partial \lambda>0\left(R_{\mathrm{III}}\right)$. 


$$
\begin{aligned}
& \mathrm{R}_{1}: \frac{\partial x^{*}}{\partial \lambda} \leq 0, \frac{\partial q^{*}}{\partial \lambda}<0 \quad \mathrm{R}_{\|}: \frac{\partial q^{*}}{\partial \lambda} \leq 0, \frac{\partial x^{*}}{\partial \lambda}>0 \quad \mathrm{R}_{\| I}: \frac{\partial q^{*}}{\partial \lambda}>0, \frac{\partial x^{*}}{\partial \lambda}>0 \\
& \underline{\beta}(\lambda) \\
& \beta^{\prime}(\lambda)
\end{aligned}
$$

Fig. 1. Spillover threshold values that limit regions $R_{\mathrm{I}}, R_{\mathrm{II}}$ and $R_{\mathrm{III}}$ for a given $\lambda$.

Figure 1 depicts the three regions for the spillovers and the impact of changing $\lambda$. Proposition 1 implies that, for demand that is convex enough, the equilibrium is always in $R_{\mathrm{I}}$ (and that a higher $\lambda$ needs a less convex demand for the result to hold). Recall that when quantities are strategic complements only $R_{\mathrm{I}}$ exists. Otherwise, the equilibrium is in $R_{\mathrm{I}}$ for a low level of spillovers only. We write the thresholds as a function of $\lambda, \beta(\lambda)$ and $\beta^{\prime}(\lambda)$, to emphasize that Proposition 1 is for a given $\lambda: \underline{\beta}(\lambda)$ is decreasing or increasing in $\lambda$ according to whether demand is concave $(\delta>0)$ or convex $(\delta<0) ; \beta^{\prime}(\lambda)$ is increasing in $\lambda$ if and only if $H$ is increasing in $\lambda$. Recall that $H$ is weakly increasing in $\lambda$ under all three model specifications: in AJ and KMZ, $H$ is independent of $\lambda$; in the CE model, $H$ is strictly increasing in $\lambda$. In those cases the effectiveness of R\&D is weakly decreasing in the degree of profit internalization $\lambda$. Both $\underline{\beta}(\lambda)$ and $\beta^{\prime}(\lambda)$ (for a given effectiveness of R\&D) are decreasing in $n .{ }^{34}$ Furthermore, $\beta^{\prime}$ is decreasing in the effectiveness of $\mathrm{R} \& \mathrm{D}\left(H^{-1}\right)$. More effective $\mathrm{R} \& \mathrm{D}$ increases $R_{\mathrm{III}}$.

We can compare these results with those reported by Leahy and Neary (1997, Prop. 3), in which there are no minority shareholdings and where $R \& D$ cooperation leads to more $\mathrm{R} \& \mathrm{D}$ and output (as in our $R_{\mathrm{III}}$ ) whenever spillovers are positive. Yet in our case, $R_{\mathrm{III}}$ obtains only when spillovers are sufficiently high. Thus the "output cooperation" induced by overlapping ownership requires sufficiently high spillovers in order to increase R\&D and output.

Finally, we are interested in analyzing the effect of $\lambda$ on each firm's profit. We have that

$$
\operatorname{sign}\left\{\pi^{* \prime}(\lambda)\right\}=\operatorname{sign}\left\{-\beta c^{\prime}\left(B x^{*}\right) \frac{\partial x^{*}}{\partial \lambda}+f^{\prime}\left(Q^{*}\right) \frac{\partial q^{*}}{\partial \lambda}\right\} .
$$

Given that $\partial x^{*} / \partial \lambda>0$ and $\partial q^{*} / \partial \lambda<0$ in $R_{\mathrm{II}}$, we can use (8) to show that -in this region $-\pi^{* \prime}(\lambda)>0$. The sign of the effect of $\lambda$ on $\pi^{*}$ is less clear in $R_{\mathrm{I}}$ (since in that region,

\footnotetext{
${ }^{34}$ For the AJ model, $\beta^{\prime}$ is decreasing in $n$ while in KMZ firm entry has no effect. In the CE model $\beta^{\prime}$ may be increasing in $n$ for $\lambda$ close to 1 .
} 
$\partial x^{*} / \partial \lambda<0$ and $\left.\partial q^{*} / \partial \lambda<0\right)$ and in $R_{\mathrm{III}}\left(\right.$ where $\partial x^{*} / \partial \lambda>0$ and $\left.\partial q^{*} / \partial \lambda>0\right)$. Nevertheless, in online appendix A.1.2 we prove the following result.

PROPOSITION 2 At the symmetric equilibrium, the profit per firm $\left(\pi^{*}\right)$ increases with $\lambda$.

According to this proposition, the positive effect on price dominates the negative effect on $\mathrm{R} \& \mathrm{D}$ in $R_{\mathrm{I}}$, and conversely in $R_{\mathrm{III}}$, so that profits in both regions rise with the extent of overlapping ownership. This means that investors and firms have always incentives to increase their interdependence. In the examples of ownership structures considered common investors to the industry have incentives to increase their share of overlapping ownership and similarly for firms to increase the overlapping ownership stake in other firms. This is so provided the agreements are binding ones, because that feature allows the parties to increase profits. ${ }^{35}$ Before proceeding with the welfare analysis, we examine the effect of $\beta$ on equilibrium values.

\subsection{Comparative statics with respect to spillovers $(\beta)$}

A sufficient (but not necessary) condition for increases in $\beta$ to raise per-firm $R \& D$ and output is that $\partial_{\beta x_{i}} \phi_{i}>0$. It is not difficult to see that $\operatorname{sign}\left\{\partial_{\beta x_{i}} \phi_{i}\right\}=\operatorname{sign}\left\{\lambda B / \tau-\chi\left(B x^{*}\right)\right\}$; here $\chi$ is the elasticity of the slope of the innovation function, which is nonnegative. For a positive $\lambda$, we have $\partial_{\beta x_{i}} \phi_{i}>0$ when the curvature (relative convexity) of the innovation function is sufficiently low. The term $\lambda B / \tau=\lambda(1+\beta(n-1)) /(1+\lambda \beta(n-1))$ increases with $\beta$ for $\lambda<1$, so it suffices that $\lambda>\chi$ (since $B / \tau=1$ for $\beta=0$ ). Our next proposition follows.

PROPOSITION 3 If the curvature $\chi$ of the innovation function is sufficiently low $(\chi<\lambda$ would be low enough), then $\partial q^{*} / \partial \beta>0$ and $\partial x^{*} / \partial \beta>0$.

We can view the following results as corollaries. In AJ (where $\chi=0$ ), stronger spillover effects raise the equilibrium values of output and $\mathrm{R} \& \mathrm{D}$. In both $\mathrm{KMZ}$ (where $\chi=1 / 2$ ) and CE (where $\chi=\alpha+1>1$ ) models it can be checked that, for $\lambda>0$, (i) $q^{*}$ increases with $\beta$ (with $\partial q^{*} / \partial \beta=0$ when $\lambda=0$ ), and (ii) $x^{*}$ increases (resp. decreases) with $\beta$ for high (resp. low) values of $\lambda$.

\footnotetext{
${ }^{35}$ Farrell and Shapiro (1990), Flath (1991), and Reitman (1994) show that unilateral incentives to implement SFI ownership structures may be lacking in Cournot competition with constant marginal costs. However, Gilo et al. (2006) show that cross-ownership arrangements facilitate tacit collusion (in the symmetric case) when the stakes are sufficiently high because they diminish incentives to deviate. For a differentiated product market with two firms, Karle et al. (2011) analyze the incentives of an investor to acquire a controlling or noncontrolling stake in a competitor.
} 
It is worth noting that $\lambda$ and $\beta$ tend to be complements in raising $x^{*}$. We have that $\partial^{2} x^{*} / \partial \lambda \partial \beta>0$ in our three model specifications according to simulations. ${ }^{36}$ A higher level of spillovers makes increasing $\lambda$ more effective in raising $x^{*}$.

\section{$5 \quad$ Welfare analysis}

Welfare in equilibrium is given by the sum of consumer surplus (CS) and industry profits:

$$
W(\lambda)=\int_{0}^{Q^{*}} f(Q) d Q-c\left(B x^{*}\right) Q^{*}-n \Gamma\left(x^{*}\right)
$$

We are interested in studying the effect of the degree of overlapping ownership $\lambda$ on welfare. Using the equilibrium conditions (2) and (3), we can write

$$
W^{\prime}(\lambda)=-\left(\Lambda f^{\prime}\left(Q^{*}\right) \frac{\partial q^{*}}{\partial \lambda}+(1-\lambda) \beta(n-1) c^{\prime}\left(B x^{*}\right) \frac{\partial x^{*}}{\partial \lambda}\right) Q^{*}
$$

An increase in overlapping ownership alters equilibrium values of quantities and $R \& D$ investments, and each additional unit of output and R\&D has social value equal to (respectively) $\Lambda\left(-f^{\prime}\left(Q^{*}\right)\right) Q^{*}$ and $(1-\lambda) \beta(n-1)\left(-c^{\prime}\left(B x^{*}\right)\right) Q^{*}$. Here Proposition 1 is useful. In $R_{\mathrm{I}}$ we have that $W^{\prime}(\lambda)<0$ because $\partial x^{*} / \partial \lambda \leq 0$ and $\partial q^{*} / \partial \lambda<0$; in $R_{\mathrm{III}}, W^{\prime}(\lambda)>0$ because $\partial x^{*} / \partial \lambda>0$ and $\partial q^{*} / \partial \lambda>0$. In $R_{\mathrm{II}}$, however, the effect of $\lambda$ on welfare is positive or negative according as whether the positive effect of overlapping ownership on R\&D does or does not dominate its negative effect on output level. Moreover, the effect of $\lambda$ on CS is positive (i.e., $\operatorname{CS}^{\prime}(\lambda)>0$ ) only when $\partial q^{*} / \partial \lambda>0$ (i.e. in $R_{\mathrm{III}}$ ). So even as consumers suffer from a higher degree of overlapping ownership in $R_{\mathrm{I}}$ and $R_{\mathrm{II}}$, it benefits them in $R_{\mathrm{III}}$. One consequence is that optimal antitrust policy will tend to be stricter under the CS standard.

\subsection{Socially optimal degree of overlapping ownership}

Let $\lambda_{\mathrm{CS}}^{o}$ and $\lambda_{\mathrm{TS}}^{o}$ denote the optimal degree of profit internalization (overlapping ownership) under the (respectively) CS and TS standard. In the three model specifications (AJ, KMZ, $\mathrm{CE}), H$ is weakly increasing in $\lambda$ and $W(\lambda)$ is single peaked. ${ }^{37}$ In the CE model, numerical simulations show that - for the parameter range in which the second-order condition (SOC)

\footnotetext{
${ }^{36}$ Furthermore, $\partial^{2} x^{*} / \partial \lambda \partial \beta$ can be shown positive when evaluated at $\lambda=\beta=0$.

${ }^{37} W(\lambda)$ is a function of one variable with only one stationary point that is a maximum (and hence a global maximum). A mild additional condition is required in KMZ. See online appendix A.2.1.
} 
and the regularity condition are satisfied $-W(\lambda)$ is strictly concave.

We know from Proposition 1 that if demand is convex enough then only $R_{\mathrm{I}}$ exists, in which case no overlapping ownership is optimal regardless of spillover levels. However, the condition for this to happen for any $\lambda(\delta \leq-n)$ is very restrictive globally since it never holds for $n \geq 2$ if the regularity condition $\Delta_{q}<0$ is required to hold for all $\lambda$ (which needs $\delta>-2$ ). We find when $\delta>-2$ (and recall that this implies that quantities are strategic substitutes for all $\lambda$ and $n$ ) that under some mild assumptions: if spillovers $\beta$ are low enough then overlapping ownership is also not optimal; and if spillovers are high enough then the level of overlapping ownership can be positive in terms of both total surplus and consumer surplus (i.e., $\lambda_{\mathrm{TS}}^{o}>0$ and $\lambda_{\mathrm{CS}}^{o}>0$ ). For intermediate values of $\beta$ we have that $\lambda_{\mathrm{TS}}^{o}>\lambda_{\mathrm{CS}}^{o}=0$. It follows that more overlapping ownership should be allowed under the total surplus standard (i.e., $\lambda_{\mathrm{TS}}^{o} \geq \lambda_{\mathrm{CS}}^{o}$ ). These results are stated formally in our next proposition.

PROPOSITION 4 Suppose that assumptions A.1-A.4 hold and let $\delta>-2$. Then if the effectiveness of $R \mathscr{E} D\left(H^{-1}\right)$ is weakly decreasing in $\lambda$ and $W(\lambda)$ is single peaked, then there are threshold values $\bar{\beta}$ and $\beta^{\prime}(0)$ (with $\bar{\beta}<\beta^{\prime}(0)$ ) such that

1. $\lambda_{\mathrm{TS}}^{o}=\lambda_{\mathrm{CS}}^{o}=0$ if $\beta \leq \bar{\beta}$;

2. $\lambda_{\mathrm{TS}}^{o}>\lambda_{\mathrm{CS}}^{o}=0$ if $\beta \in\left(\bar{\beta}, \beta^{\prime}(0)\right)$; and

3. $\lambda_{\mathrm{TS}}^{o} \geq \lambda_{\mathrm{CS}}^{o}>0$ if $\beta>\beta^{\prime}(0)$.

In all cases, $\lambda_{\mathrm{TS}}^{o} \geq \lambda_{\mathrm{CS}}^{o}$. Furthermore, whenever both $\lambda_{\mathrm{TS}}^{o}$ and $\lambda_{\mathrm{CS}}^{o}$ lie in $(0,1)$, then $\lambda_{\mathrm{TS}}^{o}, \lambda_{\mathrm{CS}}^{o}$ are strictly increasing in $\beta$.

Figure 2 depicts the critical spillover threshold values stated in Proposition 4.

$\underset{\bar{\beta}}{\lambda_{T S}^{o}=\lambda_{C S}^{o}=0 \frac{\mid}{\beta^{\prime}(0)} \quad \lambda_{T S}^{o}>\lambda_{C S}^{o}=0 \quad \lambda_{T S}^{o} \geq \lambda_{C S}^{o}>0}$

Fig. 2. Spillover threshold values that limit regions for welfare-optimal $\lambda \mathrm{s}$.

Remark 1. We have that $\bar{\beta}<1$ if $n+(n-1)(\delta+n)>H(1)$ (see Lemma 6 in online appendix A.1.2). If $\bar{\beta} \geq 1$ then $\lambda_{\mathrm{TS}}^{o}=\lambda_{\mathrm{CS}}^{o}=0$ for all $\beta \leq 1$. The threshold $\bar{\beta}$ is such that for $\beta>\bar{\beta}, W^{\prime}(0)>0$. 
Remark 2. The optimal $\lambda_{\mathrm{TS}}^{o}$ is positively associated with the effectiveness of R\&D $\left(H^{-1}\right)$. Furthermore, both $\bar{\beta}$ and $\beta^{\prime}(0)$ are decreasing in $n$ for a given effectiveness of R\&D. With more firms the scope, in terms of the range of spillovers, for welfare improving overlapping ownership increases. Furthermore, the monotonicity of $\lambda_{\text {TS }}^{o}$ and $\lambda_{\text {CS }}^{o}$ with respect to $\beta$ follows since at the optimum both $\lambda$ and $\beta$ are strategic complements in optimizing $W$ and CS (i.e. $\partial^{2} W / \partial \lambda \partial \beta>0$ and $\left.\partial^{2} \mathrm{CS} / \partial \lambda \partial \beta>0\right)$.

Remark 3. Our single-peakedness assumption on $W(\lambda)$ ensures that $\bar{\beta}$ is the minimum threshold above which total surplus increases with $\lambda$ (i.e., for which $\beta \leq \bar{\beta}$ implies $\lambda_{\text {TS }}^{o}=0$ ).

Remark 4. The assumption that $H$ is weakly increasing in $\lambda$ ensures that $\beta<\beta^{\prime}(0)$ implies $\lambda_{\mathrm{CS}}^{o}=0$ and that $\lambda_{\mathrm{TS}}^{o} \geq \lambda_{\mathrm{CS}}^{o}$. In the particular case where $\beta=\beta^{\prime}(0)$ we have that $\lambda_{\mathrm{TS}}^{o} \geq \lambda_{\mathrm{CS}}^{o} \geq 0$.

Relaxation of assumptions. If we relax the assumptions that $W(\lambda)$ be single peaked and that $H$ be monotonic in $\lambda$, then we can provide a weaker characterization of the regions where overlapping ownership is socially optimal (Proposition 5) and we are able also to characterize the extreme solution regions where $\lambda_{\mathrm{CS}}^{o}=0$ or $\lambda_{\mathrm{CS}}^{o}=\lambda_{\mathrm{TS}}^{o}=1$ (Proposition 6).

PROPOSITION 5 Let $A .1-A .4$ hold. If $\delta>-(1+n) / n$, then there exist threshold values $\underline{\beta}<\bar{\beta}<\beta^{\prime}(0)$ (where $\left.\underline{\beta}=\inf \{1 /(1+n+\Lambda \delta): \lambda \in[0,1]\}\right)$ such that: (i) $\lambda_{\mathrm{CS}}^{o}=\lambda_{\mathrm{TS}}^{o}=0$ for $\beta \leq \underline{\beta}$; (ii) $\lambda_{\mathrm{TS}}^{o}>0$ for $\beta>\bar{\beta}$; and (iii) $\lambda_{\mathrm{CS}}^{o}>0$ for $\beta>\beta^{\prime}(0)$.

Under the less restrictive assumptions we cannot ascertain what happens in the gap $(\underline{\beta}, \bar{\beta}]$. From Proposition 1 it now follows that, when $\beta \leq \underline{\beta}$, only $R_{\mathrm{I}}$ exists because $\delta>$ $-(1+n) / n$ implies that $1+n+\delta \Lambda>0$ and $\delta>-n$. The threshold $\underline{\beta}$ depends on the sign of $\delta$. If demand is concave $(\delta>0)$, then $\underline{\beta}=1 /[1+n(1+\delta)]$; if demand is convex $(\delta<0)$, then $\underline{\beta}=1 /(1+n+\delta)$. In both cases, $\underline{\beta}$ decreases with $n$ (and tends to 0 with $n$ ). ${ }^{38}$ Parts (ii) and (iii) follow as in Proposition 4: part (ii) because if $\beta>\bar{\beta}$ then $W^{\prime}(0)>0$ and so $\lambda_{\mathrm{TS}}^{o}>0$; and part (iii) because if $\beta>\beta^{\prime}(0)$ then $\partial q^{*} /\left.\partial \lambda\right|_{\lambda=0}>0$ and $\lambda_{\mathrm{CS}}^{o}>0$. (See online appendix A.1.2 for details.)

PROPOSITION 6 Under A.1-A.4, the following statements hold:

(i) $\beta<\beta_{\min }^{\prime}$ implies $\lambda_{\mathrm{CS}}^{o}=0$; and

(ii) $\beta>\beta_{\max }^{\prime}$ implies $\lambda_{\mathrm{CS}}^{o}=\lambda_{\mathrm{TS}}^{o}=1$ provided that $\beta_{\max }^{\prime} \leq 1$.

\footnotetext{
${ }^{38}$ Note that in AJ and KMZ, demand is linear and $\delta=0$; hence $\beta=1 /(1+n)$. Under CE, $\delta=-(1+\varepsilon)<0$ and so $\underline{\beta}=1 /(n-\varepsilon)$.
} 
It follows that if $\beta^{\prime}$ is independent of $\lambda$ (i.e. since $H$ is) then $\beta_{\min }^{\prime}=\beta_{\max }^{\prime}$ and we have a bang-bang solution for $\lambda_{\mathrm{CS}}^{o}$, while when $\beta^{\prime}$ is increasing in $\lambda$ (i.e. since $H$ is) then $\beta_{\min }^{\prime}=\beta^{\prime}(0)$ as in Proposition $4 .^{39}$

Proposition 6 determines when cartelization $(\lambda=1)$ is optimal in terms of both consumer and total surplus (in those cases, we are in $R_{\mathrm{III}}$ and welfare is increasing in $\lambda$ ). In AJ and $\mathrm{KMZ}$, the term $H$ is independent of $\lambda$; thus the consumer surplus solution is bang-bang under either model specification. In both specifications it is clear that if $\lambda_{\mathrm{CS}}^{o}>0$ then necessarily $\lambda_{\mathrm{TS}}^{o}=\lambda_{\mathrm{CS}}^{o}=1$. In the $\mathrm{CE}$ model, however, $H$ and $\beta^{\prime}$ are strictly increasing in $\lambda$ and hence solutions of the form $\lambda_{\mathrm{TS}}^{o}>\lambda_{\mathrm{CS}}^{o}>0$ are possible. ${ }^{40}$

The scope for a Research Joint Venture. An RJV can be understood as a situation where spillovers are fully internalized (i.e., $\beta=1$ ). If the RJV is "cartelized" then also $\lambda=1$. This arrangement can be optimal only if $R_{\mathrm{III}}$ exists for $\beta$ large (with $\beta_{\max }^{\prime} \leq 1$ ) and if $\partial q^{*} / \partial \beta>0$ and $\partial x^{*} / \partial \beta>0$ (which, by Proposition 3, holds if $\chi<1$ ). Our next corollary states the result.

COROLLARY 3 Again assume that A.1-A.4 hold. If $\beta_{\max }^{\prime} \leq 1$ and if the innovation function's curvature is not too large $(\chi<1)$, then a cartelized $R J V(\lambda=\beta=1)$ is optimal in terms of consumer and total surplus.

The assumptions of the corollary are fulfilled in the AJ and KMZ models when $R_{\mathrm{III}}$ exists $\left(\gamma b<n\right.$ and $\gamma b<1$ are needed (respectively) to ensure that $\beta_{\mathrm{AJ}}^{\prime}$ and $\beta_{\mathrm{KMZ}}^{\prime}$ are less than unity); and recall that $\chi=0$ in AJ and $\chi=1 / 2$ in KMZ. In CE, $\lambda=1$ is never socially optimal because $\beta_{\mathrm{CE}}^{\prime}(1)<1$ only if $\varepsilon<\alpha /(1+2 \alpha)$-which would contradict the regularity condition (see Table A2 in online appendix A.2.1).

Under some different conditions, an RJV with no overlapping ownership $(\lambda=0$ and $\beta=$ 1) can be socially optimal in all three models (see Proposition A1 in online appendix A.2.1). When $W(\lambda)$ is single peaked, no overlapping ownership is optimal if $\bar{\beta} \geq 1 .{ }^{41}$ In contrast with the AJ model, in both KMZ and CE we find that if $\lambda=0$ then greater R\&D spillovers reduce $\mathrm{R} \& \mathrm{D}$ expenditures $\left(\partial x^{*} / \partial \beta<0\right)$ while having no effect on output $\left(\partial q^{*} / \partial \beta=0\right)$. Although $\mathrm{R} \& \mathrm{D}$ expenditures are lower with higher $\beta$, the production costs of all firms are

\footnotetext{
${ }^{39}$ This proposition is proved by noting that $\beta^{\prime}(\lambda)$ is a continuous function on $[0,1]$ and so achieves a maximum $\left(\beta_{\max }^{\prime}\right)$ and a minimum $\left(\beta_{\min }^{\prime}\right)$ within that interval. If $\beta<\beta_{\min }^{\prime}$, then $\partial q^{*} / \partial \lambda<0$ for all $\lambda>0$ and so $\lambda_{\mathrm{CS}}^{o}=0$; if $\beta>\beta_{\max }^{\prime}$, then $\partial q^{*} / \partial \lambda>0$ for all $\lambda$. Since $\partial q^{*} / \partial \lambda>0$ implies $\partial x^{*} / \partial \lambda>0$ by equation (7), it follows that $W^{\prime}(\lambda)>0$ for all $\lambda$ by equation (9). Therefore, $\lambda_{\mathrm{CS}}^{o}=\lambda_{\mathrm{TS}}^{o}=1$ provided that $\beta_{\max }^{\prime} \leq 1$.

${ }^{40}$ In the CE case, CS is globally concave in $\lambda$ when $B>\left.H(\beta)\right|_{\lambda=0}$.

${ }^{41}$ Satisfying that inequality requires $\gamma b \geq n^{2}$ in AJ, $\gamma b \geq n$ in KMZ, and an involved condition in CE.
} 
also lower. In both cases, the greater R\&D spillover's negative effect on R\&D expenditures is dominated by its positive effect on the innovation function; as a result, $\beta=1$ is also socially optimal.

\subsection{Comparative statics by model}

We are interested in the comparative statics of the regions determining the scope for socially efficient overlapping ownership as described in Proposition 4. We are also interested in the comparative statics on $\lambda_{\mathrm{CS}}^{o}$ and $\lambda_{\mathrm{TS}}^{o}$ in the specified models. Table A3 reports the spillover thresholds for AJ, KMZ and CE models.

Comparative statics on $\beta^{\prime}(0)$ and $\bar{\beta}$. The thresholds $\beta^{\prime}(0)$ and $\bar{\beta}$ are decreasing in

- the number of firms $(n)$,

- the demand elasticity $\left(b^{-1} ; \varepsilon^{-1}\right)$, and

- the innovation function's elasticity $\left(\gamma^{-1} ; \alpha\right){ }^{42}$

The results for $\beta^{\prime}(0)$ and for $\bar{\beta}$ in relation to $n$ (except in the CE model) are analytical, the others according to numerical simulations. ${ }^{43}$ In KMZ, $\beta^{\prime}(0)$ is independent of $n$.

In terms of consumer surplus, in AJ it is optimal to suppress horizontal shareholdings for any level of spillovers when firm entry is insufficient - that is, when $n<\gamma b$ (since then $\beta_{\mathrm{AJ}}^{\prime}>1$ ); in CE, suppression is optimal when $n<\varepsilon(2 \alpha+1) / \alpha$ (since $\beta_{\mathrm{CE}}^{\prime}>1$ for $n<\varepsilon(2 \alpha+1) \Lambda / \alpha)$. We find also that $\bar{\beta}$ may take values greater than 1 when there are only a few firms in the market. ${ }^{44}$ Therefore, for highly concentrated markets, no overlapping ownership should be allowed for a wide range of spillovers. The reason is that the incentives for firms to "free ride" are stronger when the number of firms increases because each firm can then appropriate the R\&D efforts of a greater number of participants. ${ }^{45}$

Comparative statics on the socially optimal degree of overlapping ownership. Our simulations generate three main findings. First, the socially optimal level of overlapping ownership increases with the size of the spillovers, with the number of firms $(n)$, and with the elasticities of demand $\left(b^{-1} ; \varepsilon^{-1}\right)$ and of the innovation function $\left(\gamma^{-1} ; \alpha\right)$. Note that larger

\footnotetext{
${ }^{42}$ Note that $b^{-1}$ and $\gamma^{-1}$ move together with the elasticities, respectively, of demand and the innovation functions.

${ }^{43}$ Values for parameters are chosen so that the regularity condition and the SOCs are satisfied.

${ }^{44}$ In particular, from Table A3 (in online appendix A.2.1) it is straightforward to show that, in a duopoly, $\bar{\beta}>1$ when $\gamma b>4$ in AJ, when $\gamma b>2$ in KMZ, and when $\alpha>2 \varepsilon /\left(\varepsilon^{2}-7 \varepsilon+6\right)$ in CE.

${ }^{45}$ In our model a high $n$ means tougher competition and more incentives to free ride.
} 
elasticities of demand and of the innovation function increase the effectiveness of R\&D, which is positively associated with $\lambda_{\mathrm{TS}}^{o}$. Second, if the objective is to maximize consumer surplus, then the comparative statics are qualitatively similar but the scope for minority shareholdings is much lower. For example, increasing the number of firms may not in itself be sufficient for consumers to benefit from overlapping ownership; in fact, this is the case in KMZ. (Table A5 in online appendix A.2.1 provides more details of the simulations.)

We next provide graphical descriptions of the simulation results, first in the CE model and then in the AJ and KMZ models. We have made available an application program for readers to perform their own simulations. ${ }^{46}$

Constant elasticity model (Figure 3). When the number of firms is small (less than five, in our example), it is never optimal to allow minority ownership interests (since then the equilibrium is in $R_{\mathrm{I}}$ ). As the spillover effects and the number of firms increase, $\lambda_{\text {TS }}^{o}$ also increases; however, any increase in $\lambda_{\mathrm{CS}}^{o}$ is considerably smaller. The equilibrium is then in $R_{\mathrm{II}}$, where firms benefit and consumers suffer from a higher degree of overlapping ownership (because output is lower). Even so, the overall effect on welfare of increasing $\lambda$ is positive because the positive effect on $x^{*}$ dominates the negative impact on $q^{*}$. Finally, we discover that raising $\lambda$ slightly may be optimal from the consumer's standpoint when the number of firms in the market is sufficiently large (since then the equilibrium is in $R_{\mathrm{III}}$ ).

Optimal degree of overlapping ownership (TS and CS standard)

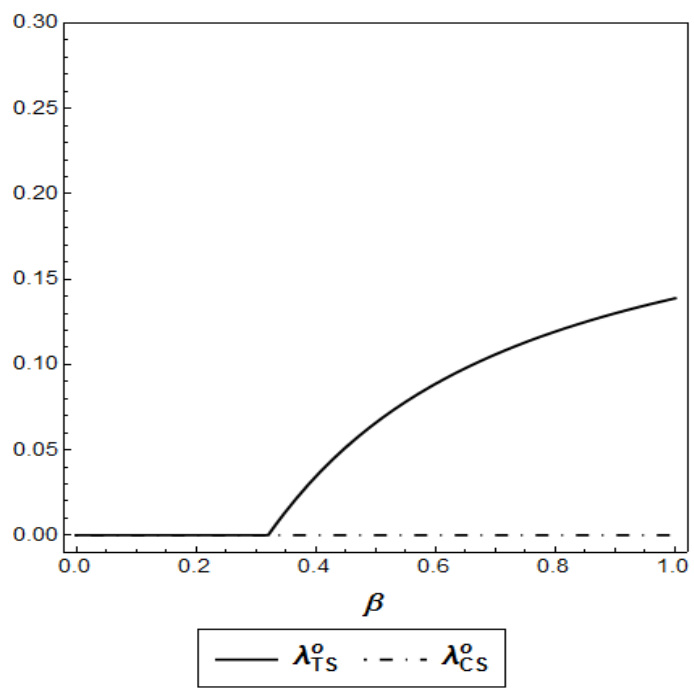

Fig. 3a. Constant elasticity model.

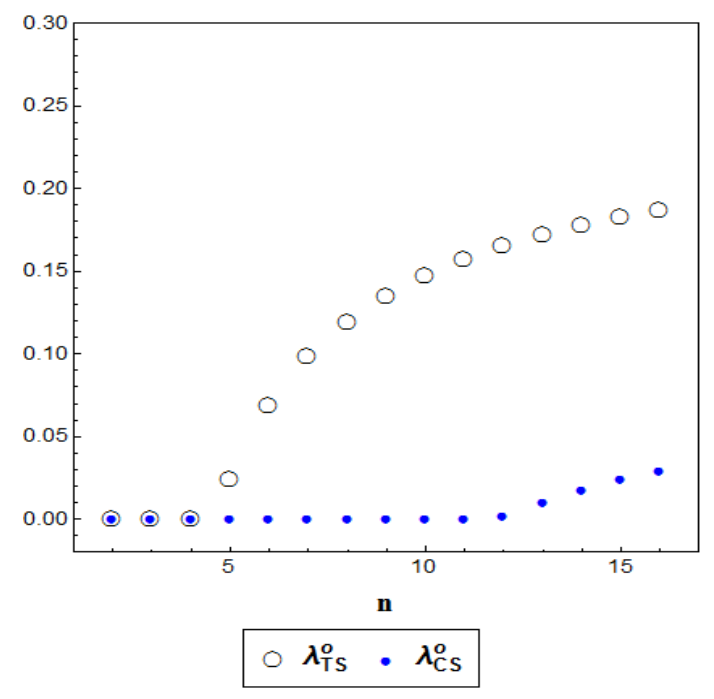

Fig. 3b. Constant elasticity model. $(\alpha=0.1, \varepsilon=0.8, \sigma=\kappa=1, \beta=0.8)$

AJ (Figure 4) and KMZ models. Figure 4a plots $\lambda_{\mathrm{TS}}^{o}$ increasing smoothly with $\beta$ after

\footnotetext{
${ }^{46}$ See www.angelluislopez.net
} 
$\bar{\beta}=0.4$ and up to $\beta^{\prime}=0.91$ where $\lambda_{\mathrm{CS}}^{o}$ jumps to 1 . In online appendix A.2.1 we can see a snapshot of our app that illustrates the simulation for $\beta=0.5$ and $n=6$. In this case the welfare translation of the increase in $\lambda$ shows in a decreasing consumer surplus and increasing per-firm profit that results in an interior solution for welfare $\lambda_{\mathrm{TS}}^{o}>0$. Figure $4 \mathrm{~b}$ shows that $\lambda_{\text {TS }}^{o}$ increases with $n$, and $\lambda_{\mathrm{CS}}^{o}$ does jump to 1 only if $n$ is sufficiently large (our example, where $\beta=0.8$, requires $n>6$ ).

Optimal degree of overlapping ownership (TS and CS standard)

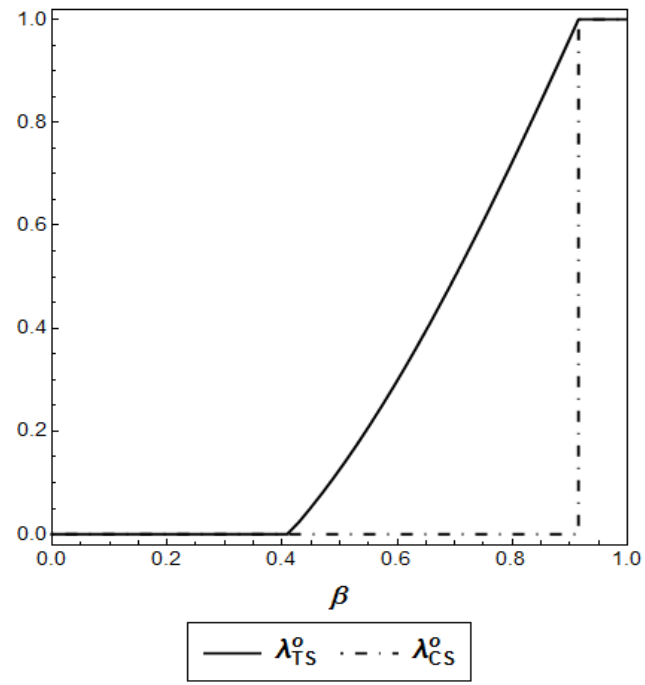

Fig. 4a. AJ model.

$(\gamma=8.5, n=6, b=0.6$. $)$

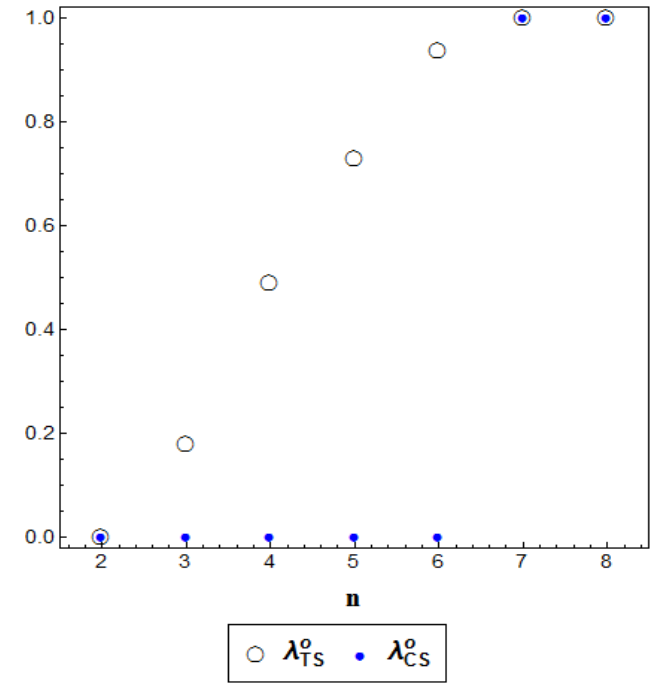

Fig. 4b. AJ model.

$(\gamma=7, \beta=0.8, b=0.6$.

Figures for the KMZ model are presented in online appendix A.2.1. In KMZ, increasing $n$ affects neither $\beta^{\prime}$ nor (as a result) $\operatorname{sign}\left\{\operatorname{CS}^{\prime}(\lambda)\right\}$. Therefore, in contrast to AJ, where for a sufficiently large number of firms we may have $\lambda_{\mathrm{CS}}^{o}=1$, in KMZ for a given $\beta<\beta_{\mathrm{KMZ}}^{\prime}$, we have $\lambda_{\mathrm{CS}}^{o}=0$ irrespective of the number of firms. Furthermore, in KMZ although $\lambda_{\mathrm{TS}}^{o}$ also increases with $n$, its rate of change decreases with $n$ (see Fig. A6b where $\lambda_{\mathrm{TS}}^{o}$ converges to a value below one when $n$ increases).

\section{Two-stage model}

We extend the "simultaneous action" (static) model of R\&D investment to a strategic commitment (two-stage) model and find that our results are (with some caveats) robust to this extension. In the first stage, every firm $i$ commits to investing an amount $x_{i}$ into R\&D. In the second stage - and for given observable level of R\&D expenditures - firms compete in the product market. We solve for the model's subgame-perfect equilibrium as a function 
of $\lambda$.

\subsection{Equilibrium and strategic effects}

Let $\mathbf{x}=\left[x_{1}, x_{2}, \ldots, x_{n}\right]$ be the first-stage $\mathrm{R} \& \mathrm{D}$ profile and let $\mathbf{q}=\left[q_{1}, q_{2}, \ldots, q_{n}\right]$ be the second-stage output profile. Let $q_{i}^{*}(\mathbf{x})$ denote firm $i$ 's (interior) output equilibrium value of the second-stage game associated with the $\mathrm{R} \& \mathrm{D}$ profile $\mathbf{x}$. Then, for all $i$, we have

$$
\frac{\partial}{\partial q_{i}} \phi_{i}\left(\mathbf{q}^{*}(\mathbf{x}), \mathbf{x}\right)=0
$$

In the first stage, the first-order necessary conditions for an interior equilibrium are (for $i \neq j$ and $i, j=1,2, \ldots, n)$

$$
\frac{\partial}{\partial x_{i}} \phi_{i}\left(\mathbf{q}^{*}(\mathbf{x}), \mathbf{x}\right)+\sum_{j \neq i} \frac{\partial}{\partial q_{j}} \phi_{i}\left(\mathbf{q}^{*}(\mathbf{x}), \mathbf{x}\right) \frac{\partial}{\partial x_{i}} q_{j}^{*}(\mathbf{x})=0
$$

The equilibrium $R \& D$ profile $\mathbf{x}^{*}$ is characterized by the system of equations (10) and (11) provided the second-order conditions hold. Let $\mathbf{q}^{*}=\mathbf{q}^{*}\left(\mathbf{x}^{*}\right)$; then $\left\{\mathbf{x}^{*}, \mathbf{q}^{*}\right\}$ is the subgameperfect equilibrium path of the two-stage game. The second term in equation (11) is the strategic effect on profits of investment. Evaluating at a symmetric equilibrium, where $q_{i}^{*}=q^{*}$ and $x_{i}^{*}=x^{*}$ for all $i$, it is easy to see that $\partial \phi_{i} / \partial q_{j}<0, j \neq i$, but the sign of $\partial q_{j}^{*} / \partial x_{i}$ is ambiguous:

$$
\operatorname{sign}\left\{\frac{\partial q_{j}^{*}}{\partial x_{i}}\right\}=\operatorname{sign}\{\beta-\tilde{\beta}(\lambda)\}, \text { where } \tilde{\beta}(\lambda) \equiv \frac{\partial_{q_{i} q_{j}} \phi_{i}}{\partial_{q_{i} q_{i}} \phi_{i}}=\frac{n(1+\lambda)+\Lambda \delta}{2 n+\Lambda \delta} .
$$

Note that the threshold $\tilde{\beta} \in(0,1]$ depends only on $\lambda, n$, and $\delta$. The inequality $\tilde{\beta}(\lambda)>$ 0 holds only if production decisions are strategic substitutes (i.e., only if $\partial_{q_{i} q_{j}} \phi_{i}<0$ ). Furthermore, $\tilde{\beta}(\lambda)<1$ for $\lambda<1$ and $\tilde{\beta}(\lambda) \rightarrow 1$ as $\lambda \rightarrow 1$.

We can also conduct comparative statics on the threshold value $\tilde{\beta}(\lambda)$. Under assumption A.1 and from the expression for $\tilde{\beta}$, it is straightforward to show the following result which highlights the crucial role played by demand curvature $\delta$.

LEMMA 3 For $\lambda<1$, the threshold $\tilde{\beta}$ : decreases (resp. increases) with $n$ if demand is concave (resp. convex); increases with $\lambda$ if $\delta>-2$; and increases with $\delta$.

When the stability condition in output is satisfied $\left(\Delta_{q}<0\right)$, we have $\partial q_{i}^{*} / \partial x_{i}>0$. So if a firm increases its investment in $R \& D$ in the first stage, then it will increase its output in 
the second stage. At the same time we have that $\partial q_{j}^{*} / \partial x_{i}>0$ when quantities are strategic complements (since then $\tilde{\beta}<0$ ). In the case of strategic substitutes, $\partial q_{j}^{*} / \partial x_{i}>0$ only if $\beta>\tilde{\beta}(\lambda)$. When a firm increases the amount invested in $\mathrm{R} \& \mathrm{D}$, it exerts two opposite effects on the output decision of rival firms. There is a positive effect because rival firms become more efficient owing to the presence of spillovers. Yet there is also a negative effect because the reaction of rivals to firm $i$ 's higher quantity is to reduce their own output via competing in the market for strategic substitutes. If spillover effects are strong enough that $\beta>\tilde{\beta}(\lambda)$, then the positive effect outweighs the negative effect; this outcome implies that $\partial q_{j}^{*} / \partial x_{i}>0$.

We can show (using A.1) that the strategic effect of investment, at a symmetric equilibrium, is as follows: ${ }^{47}$

$$
\begin{aligned}
\psi & \equiv(n-1) \frac{\partial \phi_{i}}{\partial q_{j}} \frac{\partial q_{j}^{*}}{\partial x_{i}}=-(n-1) c^{\prime}\left(B x^{*}\right) q^{*} \omega(\lambda)(\tilde{\beta}(\lambda)-\beta), \text { where } \\
\omega(\lambda) & =\frac{\Lambda}{n}\left[\frac{2 n+\Lambda \delta}{n+\Lambda(1+\delta)}\right]>0
\end{aligned}
$$

Hence we may write the FOC (11) for $\lambda \in[0,1)$ as

$$
-c^{\prime}\left(B x^{*}\right)[\tau+(n-1) \omega(\lambda)(\tilde{\beta}(\lambda)-\beta)] q^{*}-\Gamma^{\prime}\left(x^{*}\right)=0 .
$$

Since $\partial \phi_{i} / \partial q_{j}<0$, it follows that

$$
\operatorname{sign}\{\psi\}=-\operatorname{sign}\left\{\partial q_{j}^{*} / \partial x_{i}\right\}=\operatorname{sign}\{\tilde{\beta}(\lambda)-\beta\} .
$$

Thus the strategic effect $\psi$ is positive if production decisions are strategic substitutes and if $\beta<\tilde{\beta}$. In this case, there are incentives to overinvest because increasing investment reduces the rival's output. Then, as shown by Leahy and Neary (1997, Prop. 1) for $\lambda=0$, equations (10) and (14) together imply that output and R\&D are higher in the two-stage model than in the static model. ${ }^{48}$ Since each firm expects a higher first-stage investment in R\&D to reduce the second-stage output of rival firms, each firm is then led to increase their first-stage R\&D investments, which in turn boosts output in the second stage $\left(\partial q_{i}^{*} / \partial x_{i}>0\right)$. Observe that $\tilde{\beta}(1)=1$ : if there is no RJV $(\beta<1)$ then, for high levels of $\lambda$, the strategic effect is always

\footnotetext{
${ }^{47}$ The stability condition, $\Delta_{q}<0$, requires that $n+\Lambda(1+\delta)>0$ and implies that $2 n+\Lambda \delta>0$. Therefore, $\omega(\lambda)>0$.

${ }^{48}$ This result is derived under assumptions yielding a unique symmetric equilibrium and such that the two models' respective profit functions satisfy the Seade stability condition with respect to R\&D - namely, that the marginal profit of each firm with respect to $R \& D$ must decrease with a uniform increase in $R \& D$ by all firms.
} 
positive $(\beta<\tilde{\beta})$. In contrast, if $\beta$ exceeds $\tilde{\beta}$ then the strategic effect is negative; hence both output and R\&D are lower in the two-stage model than in the static model.

Remark 5. Recall that $\delta>-2$ if we want the regularity condition $\Delta_{q}<0$ for all $\lambda$. We have then that the strategic effect will tend to be positive in industries with a higher degree of overlapping ownership since then $\partial \tilde{\beta} / \partial \lambda>0$ according to Lemma 3 .

The sign of the strategic effect determines whether investment in cost reduction leads to a "top dog" or a "puppy dog" strategy in the terminology of Fudenberg and Tirole (1984). In the first case there is overinvestment and in the second underinvestment in relation to the simultaneous move case.

\subsection{Comparative statics with respect to $\lambda$}

Next we analyze how the degree of overlapping ownership affects the decisions on output and R\&D that are made in equilibrium. By using (12) and by totally differentiating the system formed by (10) and (11) before evaluating it at a symmetric equilibrium, we can solve both for $\partial q^{*} / \partial \lambda$ and for $\partial x^{*} / \partial \lambda$ under regularity conditions. Let $s(\lambda) \equiv \omega(\lambda)(\tilde{\beta}(\lambda)-\beta)$. We obtain the following result.

LEMMA 4 In the two-stage model:

$$
\begin{aligned}
& \operatorname{sign}\left\{\partial x^{*} / \partial \lambda\right\}=\operatorname{sign}\left\{\left(\beta+s^{\prime}(\lambda)\right) P^{\prime}(c)^{-1} n-[\tau+(n-1) s(\lambda)]\right\} ; \\
& \operatorname{sign}\left\{\partial q^{*} / \partial \lambda\right\}=\operatorname{sign}\left\{\left(\beta+s^{\prime}(\lambda)\right) B-\beta H(\beta)\right\} .
\end{aligned}
$$

Moreover, if $\partial x^{*} / \partial \lambda \leq 0$ then $\partial q^{*} / \partial \lambda<0$.

So once again we find that allowing for some additional degree of overlapping ownership will increase output only if it also boosts R\&D. From (15) we obtain that $\partial x^{*} / \partial \lambda>0$ if and only if $\beta>\underline{\beta}^{2 S}$ (see the proof of this lemma in online appendix A.1.3 for an expression for $\left.\underline{\beta}^{2 S}\right) .{ }^{49}$ We assume that there is at most a unique positive $\beta$, denoted $\beta^{2 S \prime}$, that solves the equation $\left(\beta+s^{\prime}(\lambda)\right) B=\beta H(\beta) .{ }^{50}$

\footnotetext{
${ }^{49}$ When there is no strategic effect (i.e. $\omega(\lambda)=0$ ), then $\underline{\beta}^{2 S}$ equals the corresponding expression in Proposition 1.

${ }^{50}$ In AJ there exists a unique $\beta^{2 S^{\prime}}<1$ when $n$ is sufficiently large or when $\gamma$ and $b$ are sufficiently low - and $\lambda$ is sufficiently large. In KMZ for high $\lambda$ and sufficiently low $\gamma$ and $b$, there exists a unique $\beta^{2 S^{\prime}}$ that is nearly (but still less than) 1 . In CE there seems to be no solution, in which case region $R_{\text {III }}$ does not exist.
} 
We are now in a position to derive the threshold values of spillovers that determine the sign of the effect, at equilibrium, of $\lambda$ on $\mathrm{R} \& \mathrm{D}$ and output. We have $\partial q^{*} / \partial \lambda \leq 0$ for $\beta \in\left[0, \beta^{2 S \prime}\right]$ and $\partial q^{*} / \partial \lambda>0$ for $\beta \in\left(\beta^{2 S \prime}, 1\right]$. Therefore: $R_{\mathrm{I}}$ (where $\partial x^{*} / \partial \lambda \leq 0$ and $\partial q^{*} / \partial \lambda<0$ ) occurs when $\beta \leq \underline{\beta}^{2 S} ; R_{\mathrm{II}}$ (where $\partial q^{*} / \partial \lambda \leq 0$ and $\partial x^{*} / \partial \lambda>0$ ) occurs for $\beta \in\left(\underline{\beta}^{2 S}, \beta^{2 S \prime}\right]$; and $R_{\mathrm{III}}$ (where $\partial q^{*} / \partial \lambda>0$ and $\partial x^{*} / \partial \lambda>0$ ) occurs when $\beta>\beta^{2 S \prime}$ with $\beta^{2 S \prime}<1$. These results extend Proposition 1 to the two-stage model and we can derive the threshold values for each of the model specifications considered in the paper (see online appendix A.2.2).

Our findings can be compared to those of Leahy and Neary (1997, Prop. 3). Those authors show that if cooperation happens only at the R\&D then the result is reduced output and R\&D - unless spillovers are high enough, in which case firms increase both output and R\&D. These two results correspond to regions $R_{\mathrm{I}}$ and $R_{\mathrm{III}}$, respectively. In addition, we identify region $R_{\mathrm{II}}$ : where cooperation driven by overlapping ownership leads to less output and more R\&D. Another difference is that, in Leahy and Neary's model, the spillover threshold above which cooperation leads to more output and R\&D lies strictly between 0 and 1 . In contrast, here (as in the simultaneous choice case) there is no guarantee that $R_{\mathrm{III}}$ exists; that is, $\beta^{2 S \prime}$ may lie above 1.

\subsection{Welfare}

We show that our welfare analysis is generally robust to the two-stage model. The only caveat is that the presence of a strategic effect of investment induces the firms to underinvest -puppy dog ploy- when spillovers are high (negative strategic effect). In this case the socially optimal level of overlapping ownership is higher than in the static model. This is consistent with our finding that the strategic effect will tend to be positive in industries with a higher degree of overlapping ownership.

Now we have (see the proof of Lemma 7 in online appendix A.1.3) that

$$
W^{\prime}(\lambda)=-\left\{\Lambda f^{\prime}\left(Q^{*}\right) \frac{\partial q^{*}}{\partial \lambda}+((1-\lambda) \beta-\omega(\lambda)(\tilde{\beta}(\lambda)-\beta))(n-1) c^{\prime}\left(B x^{*}\right) \frac{\partial x^{*}}{\partial \lambda}\right\} Q^{*}
$$

Hence the term $\omega(\lambda)(\tilde{\beta}(\lambda)-\beta)$ coming from the strategic effect of investment plays an important role in determining the impact of overlapping ownership on welfare. When the strategic effect is negative $(\beta>\tilde{\beta}(\lambda))$, the two-stage model behaves like the simultaneous model $\left(W^{\prime}(\lambda)<0\right.$ in $R_{\mathrm{I}}, W^{\prime}(\lambda)>0$ in $R_{\mathrm{III}}$, and $W^{\prime}(\lambda)$ either positive or negative (depending 
on $\beta$ ) in $R_{\mathrm{II}}$ ) but there are social incentives to increase more $\lambda$. In this case the impact of $\lambda$ on welfare through a change in $R \& D$ is magnified. Yet when the strategic effect is positive and spillovers are sufficiently low (though not necessarily close to zero), $W^{\prime}(\lambda)<0$ in $R_{\mathrm{II}}$ and $W^{\prime}(\lambda)$ can be positive or negative in $R_{\mathrm{I}}$ and in $R_{\mathrm{III}}$. In this case the impact of $\lambda$ on welfare through a change in $\mathrm{R} \& \mathrm{D}$ is dampened. A consequence of some interest is that, in $R_{\mathrm{III}}$ where $\partial x^{*} / \partial \lambda>0$ and $\partial q^{*} / \partial \lambda>0$, so consumer surplus increases with $\lambda$ (indeed, $\lambda=1$ is optimal for consumers) - total surplus can be decreasing in $\lambda$ for sufficiently high $\lambda .{ }^{51}$ Then, in stark contrast to the simultaneous model and owing to the strategic effect of investment, for some spillover values it may be that $\lambda_{\mathrm{CS}}^{o}=1>\lambda_{\mathrm{TS}}^{o}>0$. The resulting overinvestment increases output (and is good for consumer surplus) but comes at the cost of reducing firms' profits, reducing total surplus, and "overshooting" marginal cost reductions. We illustrate this possibility under the AJ and KMZ model specifications in Figure A12 for AJ and Figure A13 for KMZ in the online appendix A.2.2. ${ }^{52}$ Similarly as in the simultaneous case, there is a threshold value $\bar{\beta}^{2 S}$ for which $\lambda_{\text {TS }}^{o}>0$ if $\beta>\bar{\beta}^{2 S}$; the condition under which $\bar{\beta}^{2 S}<1$ is given by Lemma 7 in online appendix A.1.3. ${ }^{53}$

In summary, the welfare results of the simultaneous model are robust to the two-stage specification with the proviso that for high spillovers a higher degree of overlapping ownership should be allowed. In this case the strategic effect is negative and there are incentives to underinvest; then it pays to increase $\lambda$ in order to stimulate investment and output. ${ }^{54}$ This need not be the case for low values of spillovers, in which case the incentive is typically to overinvest.

Simulations The online appendix A.2.2 presents our simulations of the three considered models. These simulations confirm the qualitative results obtained in the static model, but with the two above mentioned caveats: (i) in the two-stage model, the socially optimal level of overlapping ownership tends to be higher when spillovers are high; and (ii) in some cases the consumer surplus standard may call for more cooperation than does the total surplus standard (i.e., $\lambda_{\mathrm{CS}}^{o}>\lambda_{\mathrm{TS}}^{o}>0$ ). We find also that as in the simultaneous case, for the three models considered, the spillover threshold over which some overlapping ownership is optimal $\bar{\beta}^{2 S}$ decreases with the number of firms, the elasticity of demand and with the elasticity of

\footnotetext{
${ }^{51}$ For $\beta<1$, we have $(1-\lambda) \beta-\left.\omega(\lambda)(\tilde{\beta}(\lambda)-\beta)\right|_{\lambda=1}=-(1-\beta)<0$.

${ }^{52}$ In $\mathrm{CE}$, as in the simultaneous model, $\lambda_{\mathrm{CS}}^{*}$ is usually zero or very close to zero.

${ }^{53}$ If the condition holds then $\left.W^{\prime}(0)\right|_{\beta=1}>0$, in which case there exists a sufficiently large spillover value for which some degree of overlapping ownership is welfare enhancing.

${ }^{54}$ Note that $\tilde{\beta}(\lambda) \rightarrow 1$ as $\lambda \rightarrow 1$ and so $\tilde{\beta}(\lambda)>\beta$ for $\lambda$ high enough and the strategic effect turns positive.
} 
the innovation function.

Summary When spillovers are above a given threshold, firms invest less in $R \& D$ and produce less in the two-stage than in the static model; hence the strategic effect of investment becomes negative. In this case, the social gains from a higher degree of overlapping ownership that induces firms to invest are even greater. We also characterize how these gains are affected by the number of firms, the extent of overlapping ownership, and the curvature of the inverse demand function. For a low level of spillovers, the strategic effect is positive and there are incentives to overinvest. Then it need no longer be true that the consumer surplus standard calls for reduced overlapping ownership in relation to the total surplus standpoint.

\section{Bertrand competition}

In this section we test the robustness of our results to Bertrand pricing with differentiated products. To advance the conclusion, the results obtained in the Cournot model are robust. Two interesting features of the Bertrand model are the following. First, the socially optimal level of overlapping ownership tends to have a U-shaped relationship with the degree of product differentiation (market spillovers). This is so since the closeness of the products has typically an ambiguous effect on the impact of $\lambda$ on $R \& D$ and output, but with positive impact for low or high market spillovers. Second, the strategic effect typically plays towards underinvestment even for moderate levels of spillovers (this is consistent with the analysis in Leahy and Neary 1997). For a fuller and detailed development of the analysis and proofs see online appendix B.

Consider an industry with $n$ differentiated products, each produced by one firm. The demand for good $i$ is given by $q_{i}=D_{i}(\mathbf{p})$ where $\mathbf{p}$ is the vector of prices.

Assumption 1B. For any product $i$, the function $D_{i}(\cdot)$ is smooth whenever positive, downward sloping, products are (strict) gross substitutes $\partial D_{i} / \partial p_{j}>0, j \neq i$, and the demand system $D(\cdot)$ is symmetric with negative definite Jacobian.

Under assumption 1B the demand system can be obtained from a representative consumer with quasilinear utility and can be inverted to obtain inverse demands. ${ }^{55}$ Furthermore, it follows that the demand for a variety when all firms set the same price (the Chamberlinian DD function) is downward sloping since the own-price effect dominates the cross-price

\footnotetext{
${ }^{55}$ See Vives (1999) Section 6.3 and pp. 144-148.
} 
effects: $v \equiv \partial D_{i} / \partial p_{i}+(n-1) \partial D_{j} / \partial p_{i}<0, j \neq i$. A fortiori, and for further reference, it follows that $v_{\lambda} \equiv \partial D_{i} / \partial p_{i}+\lambda(n-1) \partial D_{j} / \partial p_{i}<0$. The innovation function is as before; firm $i$ 's profit is given by

$$
\pi_{i}=\left(p_{i}-c\left(x_{i}+\beta \sum_{j \neq i} x_{j}\right)\right) D_{i}(\mathbf{p})-\Gamma\left(x_{i}\right) .
$$

and the objective function for the manager of firm $i$ is $\phi_{i}=\pi_{i}+\lambda \sum_{k \neq i} \pi_{k}$. The first-order conditions for an interior symmetric equilibrium $\left(p^{*}, x^{*}\right)$ yield

$$
\begin{gathered}
\frac{p^{*}-c\left(B x^{*}\right)}{p^{*}}=\frac{1}{\eta_{i}-\lambda(n-1) \eta_{i k}} ; \\
-c^{\prime}\left(B x^{*}\right) q^{*} \tau=\Gamma^{\prime}\left(x^{*}\right) .
\end{gathered}
$$

Here $\eta_{i}=-\frac{\partial D_{i}\left(\mathbf{p}^{*}\right)}{\partial p_{i}} \frac{p^{*}}{D_{i}\left(\mathbf{p}^{*}\right)}$ and $\eta_{i k}=\frac{\partial D_{k}\left(\mathbf{p}^{*}\right)}{\partial p_{i}} \frac{p^{*}}{D_{k}\left(\mathbf{p}^{*}\right)}, k \neq i$.

We assume parallel regularity conditions to the Cournot case which imply as before that (17) and (18) both have a unique symmetric solution if the conditions hold globally, and we assume that a symmetric regular equilibrium exists. We consider two leading examples corresponding, respectively, to the analog of the AJ and CE models with (symmetric) product differentiation. The demand systems of the examples can be derived from a symmetric (sub)utility function of a representative consumer on the vector $\mathbf{q}$ of the quantities of the varieties of the differentiated product. The first example follows our base specification with quasilinear utility while the second presents a robustness analysis in a CES model à la Dixit and Stiglitz (1977).

Linear example (with quasilinear utility) The demand for product $i$ is given by $D_{i}(\mathbf{p})=a-b p_{i}+m \sum_{j \neq i} p_{j}$ with $a>0, b>m>0$, assumption 1B holds, and as before $c_{i}=\bar{c}-x_{i}-\beta \sum_{j \neq i} x_{j}$ and $\Gamma(x)=(\gamma / 2) x^{2} \cdot{ }^{56}$

\footnotetext{
${ }^{56}$ This demand system can be obtained from
}

$$
U(\mathbf{q})=u_{1} \sum_{i=1}^{n} q_{i}-\frac{1}{2}\left(u_{2} \sum_{i=1}^{n} q_{i}^{2}+2 u_{3} \sum_{j \neq i} q_{i} q_{j}\right),
$$

where $u_{2}>u_{3}>0$ and $a, b$ and $m$ are a function of $u_{1}, u_{2}, u_{3}$ (Vives 1999, pp. 146-147.) In order to ensure positive outputs we assume that $u_{1}-\bar{c}>0$. The products range from independent $\left(u_{3}=0\right)$ to perfect substitutes $\left(u_{3}=u_{2}\right)$. 
Constant elasticity example (with non-quasilinear utility) The demand for product $i$ is given by

$$
D_{i}(\mathbf{p})=\frac{p_{i}^{-1-\rho /(1-\rho)}}{\sum_{j=1}^{n} p_{j}^{-\rho /(1-\rho)}} S,
$$

where $\rho \in(0,1)$, and $S$ is the total spending on the differentiated product varieties. Note that $\sigma \equiv 1 /(1-\rho)$ is the constant elasticity of substitution between any two products. As $\rho \rightarrow 1$, products become perfect substitutes $(\sigma \rightarrow \infty)$, while as $\rho \rightarrow 0$, products become independent $(\sigma \rightarrow 1)$. We have that $\eta_{i}=1+\left(1-n^{-1}\right) /\left(\rho^{-1}-1\right)$ and $\eta_{i k}=n^{-1} \rho /(1-\rho) .{ }^{57}$ As before, $c_{i}=\kappa\left(x_{i}+\beta \sum_{j \neq i} x_{j}\right)^{-\alpha}$ with $\alpha, \kappa>0$ and $\Gamma\left(x_{i}\right)=x_{i}{ }^{58}$

\subsection{Comparative statics with respect to $\lambda$}

In the Bertrand model we have that: for a given investment level, $\lambda$ has a positive effect on price because products are gross substitutes, $\partial_{\lambda p_{i}} \phi_{i}=(n-1)(p-c) \partial D_{k} / \partial p_{i}>0, k \neq i$; for a given price, $\lambda$ has a positive effect on investment as before, $\partial_{\lambda x_{i}} \phi_{i}=-\beta q(n-1) c^{\prime} \geq 0$, and again the total impact of $\lambda$ on the equilibrium values of price and $R \& D$ will depend on which of the two previous effects dominates. We have also that if $\partial x^{*} / \partial \lambda \leq 0$, then $\partial p^{*} / \partial \lambda>0$, because $\partial_{x_{i} p_{i}} \phi_{i}=-\left(\partial D_{i} / \partial p_{i}+\beta \lambda(n-1) \partial D_{k} / \partial p_{i}\right) c^{\prime}<0, k \neq i$, since $v<0$ and $\beta \lambda<1$ (price and $\mathrm{R} \& \mathrm{D}$ are substitutes for a firm). The upshot is that a similar result to Lemmata 1 and 2 can be established here (see Lemma B2 in online appendix B). We find that:

(i) $\operatorname{sign}\left\{\partial x^{*} / \partial \lambda\right\}=\operatorname{sign}\left\{\beta-P^{\prime}(c)|v| \tau v_{\lambda}^{-2} \partial D_{k} / \partial p_{i}\right\}$ where $P^{\prime}(c) \equiv d p^{*} / d c>0$ is the cost pass-through coefficient;

(ii) $\operatorname{sign}\left\{\partial p^{*} / \partial \lambda\right\}=\operatorname{sign}\{H-\beta B\}$, where $H=(1+\chi / y) / \xi$, and for $\Gamma^{\prime \prime}>0, \xi \equiv$ $\left(v_{\lambda} c^{\prime}\right)^{2} /\left(\Gamma^{\prime \prime} \partial D_{k} / \partial p_{i}\right)>0$. The difference with respect to the Cournot model is that here the expression for the relative effectiveness of $\mathrm{R} \& \mathrm{D}(\xi)$ takes into account the fact that products are differentiated (and the term $\left(f^{\prime}\right)^{-1}$ is replaced by $\left.v_{\lambda}^{2}\left(\partial D_{k} / \partial p_{i}\right)^{-1}\right)$.

We can define the three regions as in the Cournot case: $R_{\mathrm{I}}$, where $\partial x^{*} / \partial \lambda \leq 0$ and $\partial p^{*} / \partial \lambda>0 ; R_{\mathrm{II}}$ where $\partial x^{*} / \partial \lambda>0$ and $\partial p^{*} / \partial \lambda>0 ; R_{\mathrm{III}}$ where $\partial x^{*} / \partial \lambda>0$ and $\partial p^{*} / \partial \lambda<$

${ }^{57}$ The demand system is obtained from maximizing

$$
V\left(\mathbf{q}, q_{0}\right)=\left[\sum_{i=1}^{n} q_{i}^{\rho}\right]^{1 / \rho} q_{0}^{\theta},
$$

with $\rho \in(0,1), \theta>0$, and $q_{0}$ the numéraire good, subject to the budget constraint $q_{0}+\sum_{i=1}^{n} p_{i} q_{i}=Y$, where $Y$ is aggregate income. Then $S=Y /(1+\theta)$.

${ }^{58}$ In this model it is worth noting that the regularity condition require that $\lambda<1$. That is, the cartel problem is ill-defined with firms having incentives to set infinite prices. See online appendix B. 
0. Regarding $R_{\mathrm{I}}$, because of gross substitutes $\left(\partial D_{k}\left(\mathbf{p}^{*}\right) / \partial p_{i}>0, k \neq i\right)$, we can have $\partial x^{*} / \partial \lambda<0$ for all $\beta$. Regarding the critical spillover threshold that bounds $R_{\mathrm{II}}$ and $R_{\mathrm{III}}$, note that here, as in Cournot, assumption A.4 implies that the equation $H-\beta B=0$ has a unique positive solution, which again we may denote by $\beta^{\prime}(\lambda)$. It follows that for $\beta>\beta^{\prime}(\lambda)$, $\partial p^{*} / \partial \lambda<0$. Furthermore, $R_{\mathrm{III}}$ exists (because $\beta^{\prime}<1$ ) when $n>H(1)$. As before, $\beta^{\prime}$ is decreasing in the effectiveness of R\&D, $H^{-1}$.

In online appendix B we state the equivalent of Proposition 1 characterizing explicitly the regions for the linear and constant elasticity cases (see, respectively, propositions BL1 and BCE1). ${ }^{59}$ We find that the thresholds that bound the regions $R_{\mathrm{I}}, R_{\mathrm{II}}$ and $R_{\mathrm{III}}, \underline{\beta}(\lambda)$ and $\beta^{\prime}(\lambda)$ respectively, are increasing in $\lambda$ in both cases (see figures B1 and B11 in the online appendix), but while both $\underline{\beta}(\lambda)$ and $\beta^{\prime}(\lambda)$ are hump-shaped in $u_{3} / u_{2}$ in the linear case, both are decreasing in $\rho$ in the constant elasticity model. In the linear model we have that $\underline{\beta}(\lambda)=\beta^{\prime}(\lambda)=0$ both when products are independent $\left(u_{3} / u_{2}=0\right)$ or perfect substitutes $\left(u_{3} / u_{2}=1\right)$, in which case only $R_{\mathrm{III}}$ exists. This is so since when goods are independent to increase $\lambda$ always increases $x^{*}$ and $q^{*}$ since market power is already at its maximum level while when products tend to be homogeneous competition is so intense that the impact of increasing $\lambda$ in market power is small. ${ }^{60}$ In the constant elasticity model we also have similarly that $\beta(\lambda)=\beta^{\prime}(\lambda)=0$ for $\rho=1$ but not when $\rho=0$. This is so because the local monopoly solution is ill-defined as firms would like to charge an infinite price as $\rho \rightarrow 0$ (the elasticity of demand $\eta_{i}$ becomes unity). ${ }^{61}$ The comparative static results for $\beta^{\prime}$ follow since $H$ (inverse effectiveness of $\mathrm{R} \& \mathrm{D}$ ) has the same properties. The effectiveness of $\mathrm{R} \& \mathrm{D} H^{-1}$ is U-shaped in the closeness of the products as in the linear case (with $H^{-1} \rightarrow \infty$ both when products are close to independent and close to perfect substitutes). In the constant elasticity case $H^{-1}$ is always increasing in the elasticity of substitution of the varieties $\sigma=1 /(1-\rho)$. In sum, the closeness of the products (or the degree of market spillovers) has typically an ambiguous effect on the impact of $\lambda$ on $x^{*}$ and $q^{*}$, with positive impact for low or high market spillovers.

We find that $\partial^{2} x^{*} / \partial \lambda \partial \beta>0$ in the constant elasticity model and, according to simulations, also for the linear model.

\footnotetext{
${ }^{59}$ As in the Cournot model, in the linear Bertrand model, assumption A.4 is fulfilled and in the constant elasticity version we have that $H(\beta) / \beta B$ is decreasing in $\beta$.

${ }^{60}$ When $u_{3} \rightarrow u_{2}$, we are always in $R_{\text {III }}$ since then sign $\left\{\partial x^{*} / \partial \lambda\right\}=\operatorname{sign}\left\{\partial p^{*} / \partial \lambda\right\}=\operatorname{sign}\left\{\bar{c}-u_{1}\right\}<0$.

${ }^{61}$ In both the linear and constant elasticity cases the products do not have to be too close in order for the regularity conditions to hold.
} 


\subsection{Welfare analysis}

Welfare (with quasilinear utility) at a symmetric equilibrium is given by $W=U\left(\mathbf{q}^{*}\right)-$ $c\left(B x^{*}\right) n q^{*}-n \Gamma\left(x^{*}\right)$, where $\mathbf{q}^{*}$ is the equilibrium output vector and $U$ is the utility of a representative consumer, assumed to be smooth and strictly concave (with a negative definite Hessian). By differentiating with respect to $\lambda$ and from the maximization problem of the consumer we have that $p_{i}=\partial U / \partial q_{i}$, and at an equilibrium

$$
W^{\prime}(\lambda)=\left[\left(p^{*}-c\left(B x^{*}\right)\right) \frac{\partial q^{*}}{\partial \lambda}-\left(c^{\prime}\left(B x^{*}\right) B q^{*}+\Gamma^{\prime}\left(x^{*}\right)\right) \frac{\partial x^{*}}{\partial \lambda}\right] n
$$

which may be written as

$$
W^{\prime}(\lambda)=-\left[\frac{v}{v_{\lambda}} \frac{\partial p^{*}}{\partial \lambda}+(1-\lambda) \beta(n-1) c^{\prime}\left(B x^{*}\right) \frac{\partial x^{*}}{\partial \lambda}\right] n q^{*}
$$

Thus, since $v / v_{\lambda}>0$, we have as in the Cournot case that in $R_{\mathrm{I}}$, where $\partial x^{*} / \partial \lambda<0$ and $\partial p^{*} / \partial \lambda>0$ (so $\left.\partial q^{*} / \partial \lambda<0\right), W^{\prime}(\lambda)<0$; in $R_{\mathrm{II}}$, where $\partial x^{*} / \partial \lambda>0$ and $\partial p^{*} / \partial \lambda>0$ (so $\left.\partial q^{*} / \partial \lambda<0\right), W^{\prime}(\lambda) \lessgtr 0$; and in $R_{\mathrm{III}}$, where $\partial x^{*} / \partial \lambda>0$ and $\partial p^{*} / \partial \lambda<0$ (so $\partial q^{*} / \partial \lambda>0$ ), $W^{\prime}(\lambda)>0$.

It is worth noting that when products are independent, with the local monopoly problem well defined, and $\beta>0$ we have always that $\lambda_{T S}^{o}=\lambda_{C S}^{o}=1$. This is so since with local monopolies, as stated above, increasing $\lambda$ does not affect the degree of monopoly and helps internalizing the investment externality (if $\beta=0$, then $\lambda$ has no impact on total surplus or consumer surplus).

We can check that propositions 2 and 4 hold for the Bertrand linear and constant elasticity models, and that thresholds $\bar{\beta}$ (above which $\lambda_{T S}^{o}>0$ ) and $\beta^{\prime}(0)$ (above which $\lambda_{C S}^{o}>0$ ) are strictly decreasing in $n .{ }^{62}$ Furthermore, $\bar{\beta}$ in the linear case is hump-shaped in $u_{3} / u_{2}$ since $\bar{\beta}=0$ both for $u_{3} / u_{2}=0$ and $u_{3} / u_{2}=1$, while in the constant elasticity case is monotone decreasing in $\rho$ (or $\sigma=1 /(1-\rho))$ according to simulations.

The socially optimal $\lambda$ increases with $\beta$ and with $n$, and in terms of consumer surplus in the linear model, the scope for overlapping ownership is lower. Furthermore, both $\lambda_{T S}^{o}$ and $\lambda_{C S}^{o}$ have a U-shaped relationship with respect the degree of product differentiation in the linear case. Indeed, both $\lambda_{T S}^{o}$ and $\lambda_{C S}^{o}$ tend to 1 as products become independent

\footnotetext{
${ }^{62}$ In the constant elasticity case with non-quasilinear utility, from the resource constraint $q_{0}=Y-n c q-n x$, utility at a symmetric equilibrium is $V\left(\mathbf{q}^{*}, Y-n c\left(B x^{*}\right) q^{*}-n x^{*}\right)=n^{1 / \rho} q^{*}\left[S(1+\theta)-n c\left(B x^{*}\right) q^{*}-n x^{*}\right]^{\theta}$. We can derive $V^{\prime}(\lambda)$ accordingly (see online appendix B.4.2). We use the notation $\lambda_{T S}^{o}$ also for this case despite the fact that we have $V$ instead of TS.
} 
$\left(u_{3} / u_{2} \rightarrow 0\right)$ and they both increase also as they tend to perfectly homogeneous $\left(u_{3} \rightarrow u_{2}\right)$. In the constant elasticity model we have that $\lambda_{T S}^{o}$ grows always with $\rho$ (when positive) because the local monopoly solution (the case $\rho=0$ ) is ill-defined as explained. Figures B5-6 and B15 in online appendix B illustrate the examples.

We have, therefore, that the effect of the intensity of competition, as measured by the degree of product differentiation, on the optimal degree of profit internalization will typically be non-monotone. Note that with product differentiation we can increase the intensity of competition keeping the number of firms constant by increasing the substitutability of the products. This allows to isolate the effect of the degree of rivalry.

\subsection{Two-stage competition}

Let $\mathbf{p}^{*}(\mathbf{x})$ be the $n$-vector of second stage Bertrand equilibrium prices for a given $n$-vector of investment levels $\mathbf{x}$. In a parallel way to the Cournot case we have now that the FOC for investment for firm $i$ at a symmetric equilibrium is

$$
\frac{\partial}{\partial x_{i}} \phi_{i}\left(\mathbf{p}^{*}(\mathbf{x}), \mathbf{x}\right)+\psi(\mathbf{x})=0, \text { where } \psi(\mathbf{x}) \equiv(n-1) \frac{\partial}{\partial p_{j}} \phi_{i}\left(\mathbf{p}^{*}(\mathbf{x}), \mathbf{x}\right) \frac{\partial}{\partial x_{i}} p_{j}^{*}(\mathbf{x})
$$

is the strategic effect of investment. It is easy to see that $\partial \phi_{i} / \partial p_{j}>0$ for $\lambda<1$, while $\operatorname{sign}\left\{\partial p_{j}^{*} / \partial x_{i}\right\}$ is potentially ambiguous even if prices are strategic complements $\left(\partial_{p_{i} p_{j}} \phi_{i}>0\right.$, $j \neq i$ ) since for $\lambda>0, \partial^{2} \phi_{i} / \partial x_{j} \partial p_{i}$ is negative or positive depending on whether $\beta$ is high or low. ${ }^{63}$ For $\beta$ low when a rival (firm $i$ ) invests in cost reduction the cost of firm $j$ is not reduced by much and the marginal return to the manager of firm $j$, which includes the marginal profits of rivals, to raising price increases (i.e., $\partial^{2} \phi_{j} / \partial x_{i} \partial p_{j}>0$ and the price best reply of firm $j$ moves outwards). When $\beta$ is high the opposite happens. ${ }^{64}$

In short, sufficient conditions for $\partial p_{j}^{*}(\mathbf{x}) / \partial x_{i}<0$ are that $\beta$ is high and prices are strategic complements; then increasing $x_{i}$ decreases the prices of rivals because a larger $x_{i}$ shifts the price best reply of firm $j$ inwards as $\partial^{2} \phi_{j} / \partial x_{i} \partial p_{j}<0$ as well as shifting inwards also the price best reply of firm $i$ since $\partial^{2} \phi_{i} / \partial x_{i} \partial p_{i}<0$. The result is that the strategic effect is negative $(\psi<0)$ and we have puppy dog investment incentives. However, the conditions are not necessary, both in the linear and CE cases we have in fact that $\partial p_{j}^{*} / \partial x_{i}<0$ for $\lambda<1$ and any $\beta$.

\footnotetext{
${ }^{63}$ We have that $\partial^{2} \phi_{i} / \partial x_{j} \partial p_{i}=-c^{\prime}(B x)\left\{\beta\left[\partial D_{i} / \partial p_{i}+\lambda(n-1) \partial D_{k} / \partial p_{i}\right]+(1-\beta) \lambda \partial D_{k} / \partial p_{i}\right\}$.

${ }^{64}$ When $\lambda$ is low then we have $\partial^{2} \phi_{j} / \partial x_{i} \partial p_{j}<0$ even for $\beta$ not very high (indeed, for any $\beta>0$ when $\lambda=0)$.
} 
We can write the strategic effect as $\psi=-(n-1) c^{\prime}(B x) q^{*} \omega(\lambda)(\tilde{\beta}(\lambda)-\beta)$ where $\omega(\lambda)>$ 0 . In the linear and CE cases we have that $\tilde{\beta}(\lambda)<0$ and therefore $\psi<0$, but in general we may have $\tilde{\beta}(\lambda)>0$.

Welfare. From the FOCs for price and investment we obtain:

$$
W^{\prime}(\lambda)=-\left\{\frac{1}{v_{\lambda}} \frac{\partial q^{*}(\lambda)}{\partial \lambda}+[(1-\lambda) \beta-\omega(\lambda)(\tilde{\beta}(\lambda)-\beta)](n-1) c^{\prime}\left(B x^{*}\right) \frac{\partial x^{*}(\lambda)}{\partial \lambda}\right\} n q^{*}
$$

Recall that in Cournot when the strategic effect is negative (i.e., $(\tilde{\beta}(\lambda)-\beta)<0)$, the sign of the impact of $\lambda$ on welfare in each region $\left(R_{\mathrm{I}}, R_{\mathrm{II}}\right.$ and $\left.R_{\mathrm{III}}\right)$ is the same in the simultaneous and the two-stage model. This is the case also with Bertrand competition and $\beta$ high (puppy dog strategy) and also in the linear and constant elasticity models for any $\beta$.

In the linear model we obtain similar comparative statics results than in Cournot twostage: $\lambda_{T S}^{o}$ increases with $\beta$ and $n$, and in the two-stage $\lambda_{T S}^{o}$ tends to be higher than in the simultaneous model when spillovers are high. However, and unlike the Cournot model, we do not observe cases in which $\lambda_{C S}^{o}>\lambda_{T S}^{o}$. The reason is that those cases may arise in Cournot when the strategic effect is positive; in the Bertrand linear model the strategic effect is always negative. Furthermore, we do not have in Bertrand a bang-bang solution with the consumer surplus standard. ${ }^{65}$

\section{$8 R \& D$ and output cooperation}

R\&D cooperation may extend to the product market. In this situation, even with no OOAs, when firms cooperate in R\&D they may cooperate also in output and/or price. The intensity of cooperation can be measured by the "sympathy coefficient" $\lambda$; for example, a low $\lambda$ may be the result of firms' limited scope for collusion owing to a low discount factor. Note also that this parameter has an empirical counterpart in the estimation of market power because it corresponds to a constant elasticity of conjectural variation, which can be used to estimate the degree of industry cooperation. ${ }^{66}$ The partial collusion scenario is relevant given the long-standing suspicion that R\&D cooperation facilitates coordination in the product market. This outcome may reflect the existence of ancillary restraints (or of other channels

\footnotetext{
${ }^{65}$ The bang-bang solution arises when $H$ is independent of $\lambda$, so $\beta^{\prime}$ also is (as in AJ and KMZ), however in the Bertrand linear model $H$ is strictly increasing in $\lambda$ (as in the CE model).

${ }^{66}$ Michel (2016) estimates the degree of profit internalization after ownership changes in differentiated product industries. He allows each firm's objective function to depend on other firms' profits by incorporating the parameter $\lambda_{i j}$, which is the extent to which brand $i$ accounts for brand $j$ 's profits when setting the optimal brand- $i$ price.
} 
through which cooperative $R \& D$ may lead to coordination in the product market ${ }^{67}$ or the existence of multimarket contacts. ${ }^{68}$ There is also growing evidence that R\&D cooperation facilitates product market cooperation from empirical studies (Duso et al. 2014; Goeree and Helland 2010), from experiments (Suetens 2008), ${ }^{69}$ and from antitrust cases. ${ }^{70}$ There is also recent evidence that price and product space collusion may go together (Sullivan 2016).

Our analysis therefore extends the traditional framework in two directions: no separation between coordination in R\&D and output, whether because of overlapping ownership or because $\mathrm{R} \& \mathrm{D}$ cooperation naturally extends to product market cooperation; and the presence of intermediate degrees of cooperation in response to the strictness of competition policy. Antitrust authorities affect the parameter $\lambda$ by limiting cross-shareholdings; we can also interpret $\lambda$ as a measure of the intensity with which collusion is scrutinized. ${ }^{71}$ From a policy perspective, our results highlight the tension between a CS standard as proclaimed by many competition authorities and the fact that R\&D cooperation is widely allowed (and even encouraged) by those same public authorities. Whenever cooperation in R\&D extends to competition in the product market, policy must in general be much stricter if the aim is to increase consumer surplus.

\section{Concluding remarks}

The competition-reducing effect of overlapping ownership may justify policy intervention. However, some degree of overlapping ownership may actually be welfare enhancing, and may even increase consumer surplus, for an industry that exhibits sufficiently large R\&D spillovers. In the extreme, it may be socially optimal to form a cartelized RJV ("merger

\footnotetext{
${ }^{67}$ As when, for example, an RJV stipulates downstream market division for any patents that may result from the venture or when there are collateral agreements that impose cross-licensing of old patents (or a per-unit output royalty for using new patents) - since these circumstances reduce the incentives of firms to increase their output (Grossman and Shapiro 1986; Brodley 1990). The various channels through which cooperative R\&D may facilitate coordination in the product market are analyzed by Martin (1995), Greenlee and Cassiman (1999), Cabral (2000), Lambertini et al. (2002), and Miyagiwa (2009).

${ }^{68}$ See the related evidence in Parker and Röller (1997) for mobile telephony and in Vonortas (2000) for US RJVs.

${ }^{69}$ Suetens (2008) uses a two-stage duopoly model to confirm that cooperation in reducing R\&D costs facilitates price collusion. Agents engage in cooperative R\&D projects more than once, and they interact repeatedly in the product market. For both small and large spillovers this author finds that cooperativeness in the pricing stage is generally higher when subjects can make binding R\&D agreements than in the baseline treatments without the possibility of such agreements.

${ }^{70}$ Goeree and Helland (2010) gather a number of cases in the petroleum industry, the computer industry, the market for semiconductor memory, and the telecommunications sector.

${ }^{71}$ Besanko and Spulber (1989) show that, if collusive behavior is unobservable and if production costs are private information, then the antitrust authority may optimally induce some intermediate degree of collusion among firms.
} 
to monopoly"). This paper stipulates precise conditions that can be checked to see whether overlapping ownership is (or is not) improving social welfare. ${ }^{72}$ We extend the "simultaneous action" (static) model of R\&D investment to a strategic commitment model and find that our results are with some caveats robust to this extension. We find that OOAs may be welfare improving in particular when spillovers are high and investment has commitment value since in this case firms have strong incentives to underinvest. The results are robust also to a Bertrand model with differentiated products.

Antitrust scrutiny of OOAs should increase in industries with high concentration since the spillover thresholds below which OOAs are welfare-decreasing are increasing in concentration (as measured by the HHI) and with low levels of spillovers (typically industries with low levels of R\&D or, alternatively, with tight patent protection). The documented increase in concentration in the US in the recent decades and the positive statistical relationship between concentration and patents found in recent data (Grullon et al. 2017) may suggest a potential decrease in spillovers and need to tighten antitrust policy. ${ }^{73}$ The conditions for OOAs to improve welfare are typically even more restrictive under a consumer surplus standard. This fact may lead to a potential tension for competition policy since authorities adhere to a consumer surplus standard while they allow high degrees of OOAs and R\&D cooperation. Mergers may realize synergies by internalizing technological spillovers but increase concentration, which makes the anti-competitive effects of OOAs more likely. This calls for caution when advocating softer competition policy on mergers when overlapping ownership is high (e.g., Posner et al. 2016).

Finally, the scrutiny of horizontal shareholdings should distinguish according to their type. This is so because the same extent of shareholding will lead to different degrees of internalization of rivals' profits. If the regulator wants to establish a cap on the degree of internalization this will imply a more strict cap on shareholdings with proportional control than those with silent financial interests or those which are cross-shareholdings among firms.

\footnotetext{
${ }^{72}$ The extent of welfare enhancing OOAs may be fostered by a feedback effect on the degree of spillovers (see He and Huang 2017). Ghosh and Morita (2017) show how partial equity ownership may induce knowledge transfer between alliance partners.

${ }^{73}$ There is a negative relationship between spillovers and patent protection levels in a range of industries (Griliches 1990, Galasso and Schankerman 2015).
} 


\section{Appendix}

Table 4: Summary of Basic Expressions at the Symmetric Equilibrium of the Simultaneous Game

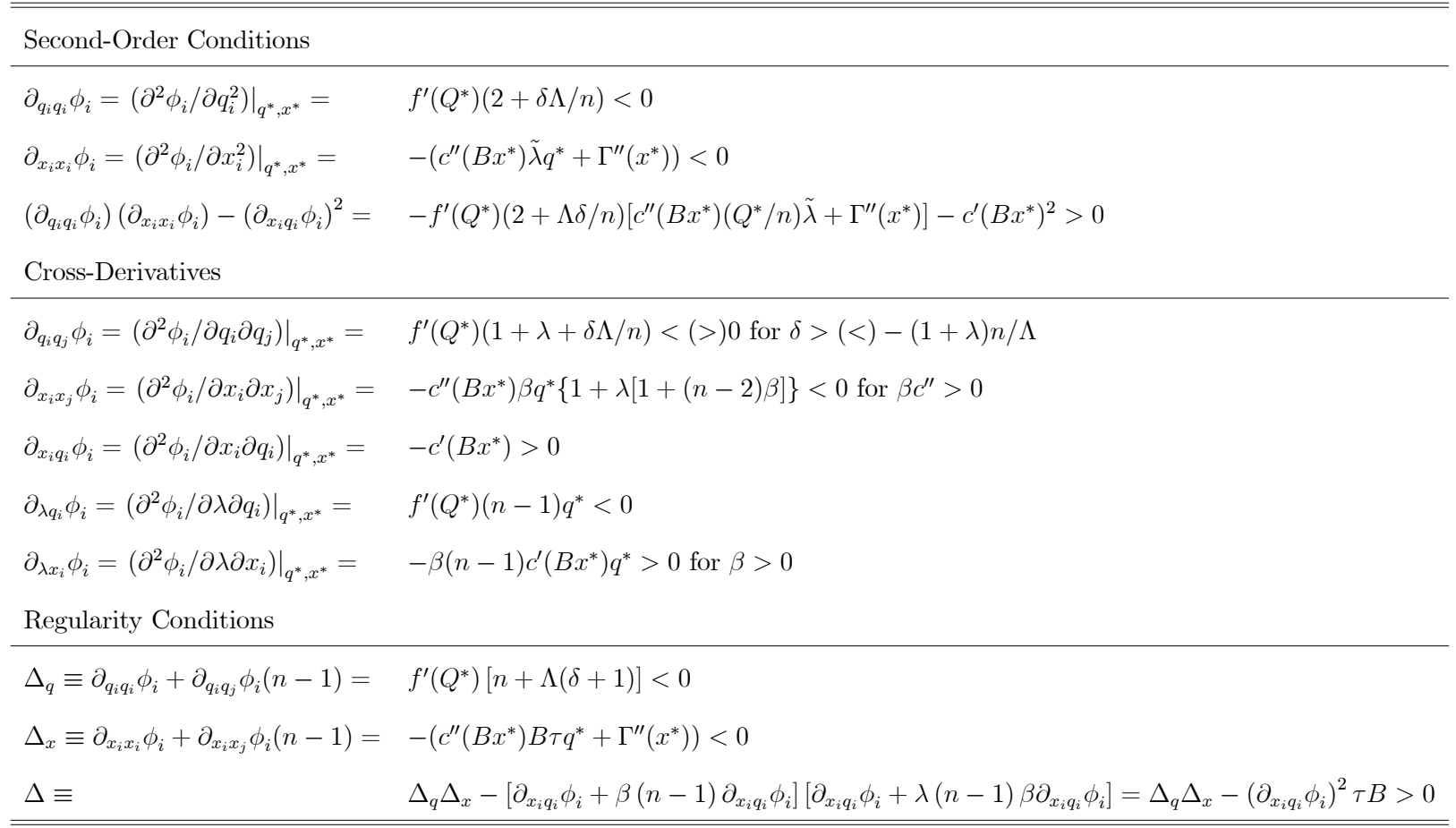

with $B \equiv 1+\beta(n-1), \Lambda \equiv 1+\lambda(n-1), \tau \equiv 1+\lambda(n-1) \beta$ and $\tilde{\lambda} \equiv 1+\lambda(n-1) \beta^{2}$.

Remark: $\Delta_{q}<0 \Leftrightarrow \delta \Lambda>-(\Lambda+n)$, whereas $\partial_{q_{i} q_{i}} \phi_{i}<0 \Leftrightarrow \delta \Lambda>-2 n$, thus $\Delta_{q}<0$ implies that $\partial_{q_{i} q_{i}} \phi_{i}<0$,

and to have $\Delta_{x}<0$ we need that $c^{\prime \prime}>0$ or $\Gamma^{\prime \prime}>0$, and therefore $\partial_{x_{i} x_{i}} \phi_{i}<0$.

The signs of the expressions follow under our assumptions.

\section{References}

[1] Aghion, P., J. Van Reenen, and L. Zingales. 2013. "Innovation and Institutional Ownership." American Economic Review 103: 277-304.

[2] Allen, J., and G. Phillips. 2000. "Corporate Equity Ownership, Strategic Alliances, and Product Market Relationships." Journal of Finance 55: 2791-2815.

[3] Amir, R. 2000. "Modelling Imperfectly Appropriable R\&D via Spillovers." International Journal of Industrial Organization 18: 1013-1032. 
[4] Anton, M., F. Ederer, M. Giné, and M. Schmalz. 2017. "Common Ownership, Competition, and Top Management Incentives." Ross School of Business working paper 1328.

[5] Azar, J., S. Raina, and M.C. Schmalz. 2016. "Ultimate Ownership and Bank Competition." Available at SSRN.

[6] Azar, J., M.C. Schmalz, and I. Tecu. (forthcoming). "Anti-competitive Effects of Common Ownership." Journal of Finance.

[7] Baker, J. 2016. "Overlapping Financial Investor Ownership, Market Power, and Antitrust Enforcement: My Qualified Agreement with Professor Elhauge." Harvard Law Review 129: 212-232.

[8] Banal-Estanol, A., J. Seldeslachts, and X. Vives. 2018. "Common Ownership -Product Market Consequences of a Shift from Active to Passive Investors." Mimeo.

[9] Besanko, D., and D.F. Spulber. 1989. "Antitrust Enforcement under Asymmetric Information." Economic Journal 99: 408-25.

[10] Bloom, N., M. Schankerman, and J. Van Reenen. 2013. "Identifying Technology Spillovers and Product Market Rivalry." Econometrica 81: 1347-1393.

[11] Blume, M.E., and B. Keim. 2014. "The Changing Nature of Institutional Stock Investing". Working paper.

[12] Bresnahan, T.F., S.C. Salop. 1986. "Quantifying the Competitive Effects of Production Joint Ventures." International Journal of Industrial Organization 4: 155-175.

[13] Brito, D., R. Ribeiro, and H. Vasconcelos. 2014. "Measuring Unilateral Effects in Partial Horizontal Acquisitions," International Journal of Industrial Organization 33: 22-36.

[14] Brito, D., Osório, A., Ribeiro, R., and H. Vasconcelos. (forthcoming). "Unilateral Effects Screens for Partial Horizontal Acquisitions: The Generalized HHI and GUPPI." International Journal of Industrial Organization.

[15] Brodley, J. 1990. "Antitrust Law and Innovation Cooperation." Journal of Economic Perspectives 4: 97-112. 
[16] Bulow, J., and P. Pfleiderer. 1983. "A Note on the Effect of Cost Changes on Prices." Journal of Political Economy 91: 182-185.

[17] Bushee, B. 1998. "The Influence of Institutional Investors on Myopic R\&D Investment Behavior." The Accounting Review 73:305-333.

[18] Cabral, L. 2000. "R\&D Cooperation and Product Market Competition." International Journal of Industrial Organization 18: 1033-1047.

[19] CEA. 2016. "Benefits of Competition and Indicators of Market Power." Council of Economic Advisers Issue Brief. April.

[20] Dasgupta, P., and J. Stiglitz. 1980. "Industrial Structure and the Nature of Innovative Activity." Economic Journal 90: 266-93.

[21] d'Aspremont, C., and A. Jacquemin. 1988. "Cooperative and Noncooperative R\&D in Duopoly with Spillovers." The American Economic Review 78: 1133-1137.

[22] De Loecker, J., and J. Eeckhout. 2017. "The Rise of Market Power and the Macroeconomic Implications". Working paper.

[23] Dietzenbacher, E., B. Smid, and B. Volkerink. 2000. "Horizontal Integration in the Dutch Financial Sector," International Journal of Industrial Organization 18: 12231242.

[24] Dixit, A., and J. Stiglitz. 1977. "Monopolistic Competition and Optimum Product Diversity." American Economic Review 67: 297-308.

[25] Dixit, A. 1986. "Comparative Statics for Oligopoly." International Economic Review 27: $107-122$.

[26] Duso, T., L-H. Röller, and J. Seldeslachts. 2014. "Collusion Through Joint R\&D: An Empirical Assessment." The Review of Economics and Statistics 96: 349-370.

[27] Edgeworth, F. Y. 1881. Mathematical Physics: An Essay on the Application of Mathematics to the Moral Sciences. (Kegan Paul, London).

[28] Elhauge, E. 2016. "Horizontal Shareholding." Harvard Law Review 109: 1267-1317. 
[29] Elhauge E. 2017. "Tackling horizontal shareholding: an update and extension to the Sherman Act and EU competition law." Background Paper for 128th meeting of the OECD Competition Committee.

[30] Eng, L.L., and M. Shackell. 2001. "The Implications of Long-Term Performance Plans and Institutional Ownership for Firms' Research and Development (R/D\&D) Investments." Journal of Accounting, Auditing \& Finance 16: 117-139.

[31] European Commission [EC]. 2014. "White Paper: Towards More Effective EU Merger Control." Brussels, 9.7.2014. COM(2014) 449 final.

[32] European Commission[EC] Decision.2017. Case M.7392, Dow/Dupont, 27 march.

[33] Farrell, J., and C. Shapiro. 1990. "Asset Ownership and Market Structure in Oligopoly." RAND Journal of Economics 21: 275-292.

[34] Fee, C. E., C. J. Hadlock, and S. Thomas. 2006. "Corporate Equity Ownership and the Governance of Product Market Relationships." Journal of Finance 61:1217-1251.

[35] Flath, D. 1991. "When is it Rational for Firms to Acquire Silent Interests in Rivals?" International Journal of Industrial Organization 9: 573-584.

[36] Fudenberg, D., and J. Tirole. 1984. "The Fat-Cat Effect, the Puppy-Dog Ploy, and the Lean and Hungry Look." The American Economic Review 74: 361-366.

[37] Galasso, A., and M. Schankerman. 2015. "Patents and Cumulative Innovation: Causal Evidence from the Courts." Quarterly Journal of Economics 130: 317-369.

[38] Geng, H., H. Hau, and S. Lai. 2016. "Technological Progress and Ownership Structure." SFI research paper series $15-39$.

[39] Ghosh, A., and H. Morita. 2017. "Knowledge Transfer and Partial Equity Ownership." Rand Journal of Economics 48: 1044-1067.

[40] Gilbert, R. 2006. Looking for Mr. Schumpeter: Where Are We in the CompetitionInnovation Debate?, in Adam B. Jaffe, Josh Lerner and Scott Stern (eds), Innovation Policy and the Economy 6: 159-215. The MIT Press.

[41] Gilbert, R., and H. Greene. 2015. "Merging Innovation into Antitrust Agency Enforcement of the Clayton Act." The George Washington Law Review 83: 1919-1947. 
[42] Gilo, D. 2000. "The Anticompetitive Effect of Passive Investment." Michigan Law Review 99: 1-47.

[43] Gilo, D., Y. Moshe, and Y. Spiegel. 2006. "Partial Cross Ownership and Tacit Collusion." RAND Journal of Economics 37: 81-99.

[44] Goeree, M., and E. Helland. 2010. "Do Research Joint Ventures Serve a Collusive Function?" Working Paper 448, Institute for Empirical Research in Economics.

[45] Gramlich, J., and Grundl, S. 2017. "Estimating the Competitive Effects of Common Ownership." Mimeo.

[46] Greenlee, P., and B. Cassiman. 1999. "Product Market Objectives and the Formation of Research Joint Ventures." Managerial and Decision Economics 20: 115-130.

[47] Griliches, Z. 1990. "Patent Statistics as Economic Indicators: A Survey." Journal of Economic Literature 28: 1661-1707.

[48] Grossman, M., and C. Shapiro. 1986. "Research Joint Ventures: An Antitrust Analysis." Journal of Law, Economics, \& Organization 2: 315-337.

[49] Grullon, G., Y. Larkin, and R. Michaely. 2017. "Are US Industries Becoming More Concentrated". Mimeo.

[50] Gutiérrez, G. and T. Philippon. 2016. "Investment-less Growth: An Empirical Investigation." Mimeo.

[51] Hansen R. and J. Lott, Jr. 1996. "Externalities and corporate objectives in a world with diversified shareholder/consumers." Journal of Financial and Quantitative Analysis 31:43-68.

[52] He, J.J., and J. Huang. 2017. "Product Market Competition in a World of Cross Ownership: Evidence from Institutional Blockholdings." Review of Financial Studies 30: $2674-2718$.

[53] Heim, M., K. Hüschelrath, and Y. Spiegel. 2017. "Minority Share Acquisitions and Collusion: Evidence from the Introduction of National Leniency Programs." Mimeo. 
[54] Investment Company Institute. 2017. Fact Book - A Review of Trends and Activities in the Investment Company Industry. Retrieved from Investment Company Institute Website: https://www.ici.org/pdf/2017_factbook.pdf

[55] Kamien, M., E. Muller, and I. Zang. 1992. "Research Joint Ventures and R\&D Cartels." The American Economic Review 82: 1293-1306.

[56] Karle, H., T.J. Klein, and K. Stahl. 2011. "Ownership and Control in a Competitive Industry." CESifo Working Paper No. 3380.

[57] Kennedy, P., D. O’Brien, M. Song, and K. Whaerer. 2017. "The Competitive Effects of Common Ownership: Economic Foundations and Empirical Evidence." Mimeo.

[58] Lambertini, L., S., Poddar and D. Sasaki. 2002. "Research Joint Ventures, Product Differentiation and Price Collusion." International Journal of Industrial Organization 20: 829-854.

[59] Leahy, D., and P. Neary. 1997. "Public Policy towards R\&D in Oligopolistic Industries." The American Economic Review 87: 642-662.

[60] Liang, L. M. 2016. "Common Ownership and Executive Compensation." Mimeo.

[61] Martin, S. 1995. "R\&D Joint Ventures and Tacit Product Market Collusion." European Journal of Political Economy 11: 733-741.

[62] Michel, C. 2016. "Identification and Estimation of Intra-Firm and Industry Competition via Ownership Change." Mimeo.

[63] Miyagiwa, K. 2009. "Collusion and Research Joint Ventures." The Journal of Industrial Economics 57: 768-784.

[64] Nain, A., and Y. Wang. 2016. "The Product Market Impact of Minority Stake Acquisitions," Management Science 64: 825-844.

[65] Norbäck, P-J., Persson, L. and Tag, J. 2018. "Private Equity Buyouts: Anti-or Procompetitive?" Antitrust Chronicle 1: 32-36.

[66] Parker, P. M., and L-H. Röller. 1997. "Collusive Conduct in Duopolies: Multimarket Contact and Cross-Ownership in the Mobile Telephone Industry." Rand Journal of Economics 28: 304-322. 
[67] Posner, E., F. Scott Morton, and E. G. Weyl. 2016. "A Proposal to Limit the AntiCompetitive Power of Institutional Investors." Mimeo.

[68] Reitman, D. 1994. "Partial Ownership Arrangements and the Potential for Collusion." The Journal of Industrial Economics 42: 313-322.

[69] Reynolds, R.J., B.R. Snapp. 1986. "The Competitive Effects of Partial Equity Interests and Joint Ventures." International Journal of Industrial Organization 4: 141-153.

[70] Rock, E. B., and D. L. Rubinfeld. 2017. "Antitrust for Institutional Investors." NYU School of Law, WP NO.17-23.

[71] Salop, S.C., and D.P. O’Brien. 2000. "Competitive Effects of Partial Ownership: Financial Interest and Corporate Control." Antitrust Law Journal 67: 559-614.

[72] Schmalz, M.C. 2018. "Common-Ownership Concentration and Corporate Conduct." Annual Review of Financial Economics 10: 1-31.

[73] Shelegia, S., and Y. Spiegel. 2012. "Bertrand Competition when Firms Hold Passive Ownership Stakes in One Another." Economics Letters 114: 136-138.

[74] Spence, M. 1984. "Cost Reduction, Competition and Industry Performance." Econometrica 52: 101-121.

[75] Suetens, S. 2008. "Does R\&D Cooperation Facilitate Price Collusion? An Experiment." Journal of Economic Behavior \& Organization 66: 822-836.

[76] Sullivan, J. 2016. "The Ice Cream Split: Empirically Distinguishing Price and Product Space Collusion." Mimeo.

[77] Vives, X. 1999. Oligopoly Pricing: Old Ideas and New Tools. Cambridge, MA: MIT Press.

[78] Vives, X. 2008. "Innovation and Competitive Pressure." The Journal of Industrial Economics 56: 419-469.

[79] Vonortas, N.S. 2000. "Multimarket Contact and Inter-Firm Cooperation in R\&D." Journal of Evolutionary Economics 10: 243-271.

[80] Weyl, E.G., and M. Fabinger. 2013. "Pass-Through as an Economic Tool: Principles of Incidence under Imperfect Competition." Journal of Political Economy 121: 528-583. 
[81] Wilkinson, L.A., and J.L. White. 2007. "Private Equity: Antitrust Concerns with Partial Acquisitions." Antitrust 21: 28-34. 


\title{
Online appendix to Overlapping Ownership, R\&D Spillovers and Antitrust Policy
}

\author{
Ángel L. López and Xavier Vives
}

July 2018

\section{Contents}

A Proofs and the three model specifications 2

A.1 General model: proofs . . . . . . . . . . . . . . . . . . . . 2

A.1.1 Overlapping ownership and $\lambda \ldots \ldots \ldots \ldots \ldots$

A.1.2 Simultaneous model . . . . . . . . . . . . . . . . . . . . . 5

A.1.3 Two-stage model . . . . . . . . . . . . . . . . . . . . . 13

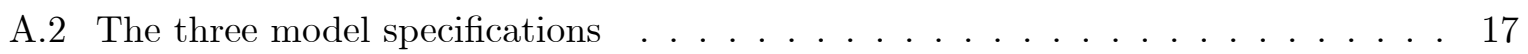

A.2.1 Simultaneous model . . . . . . . . . . . . . . . . . . . . 18

A.2.2 Two-stage model . . . . . . . . . . . . . . . . . 30

$\begin{array}{ll}\text { B Bertrand competition with differentiated products } & 37\end{array}$

B.1 Framework and equilibrium . . . . . . . . . . . . . . . . . 37

B.2 Simultaneous model . . . . . . . . . . . . . . . . . . . . 38

B.2.1 Comparative statics with respect to $\lambda \ldots \ldots \ldots \ldots$

B.2.2 Welfare analysis . . . . . . . . . . . . . . . . 44

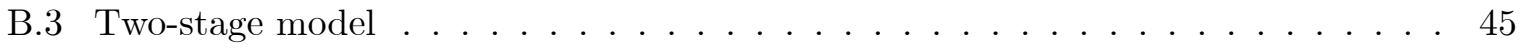

B.4 Model specifications . . . . . . . . . . . . . . . . . . . . . . . 49

B.4.1 Linear model . . . . . . . . . . . . . . . . . . . . . . . . . . . . . . . 49

B.4.2 Constant elasticity model . . . . . . . . . . . . . . . . 66

In Appendix A we provide some technical details and proofs of results in the paper in Section A.1, and analysis and results for the three model specifications in Section A.2. In Appendix B we develop the Bertrand model with product differentiation. 


\section{A Proofs and the three model specifications}

\section{A.1 General model: proofs}

\section{A.1.1 Overlapping ownership and $\lambda$}

Common ownership Consider an industry with $n$ firms and $I \geq n$ investors; we let $i$ and $j$ index (respectively) investors and firms. The share of firm $j$ owned by investor $i$ is $v_{i j}$, and the parameter $\zeta_{i j}$ captures the extent of $i$ 's control over firm $j$. The total (portfolio) profit of investor $i$ is $\pi^{i}=\sum_{k} v_{i k} \pi_{k}$, where $\pi_{k}$ are the profits of portfolio firm $k$. The manager of firm $j$ takes into account shareholders' incentives (through the control weights $\zeta_{i j}$ ) and maximizes a weighted average of the shareholders' portfolio profits:

$$
\sum_{i=1}^{I} \zeta_{i j} \pi^{i}=\left(\sum_{i=1}^{I} \zeta_{i j} v_{i j}\right) \pi_{j}+\sum_{i=1}^{I} \zeta_{i j} \sum_{k \neq j}^{n} v_{i k} \pi_{k} .
$$

It is immediate dividing by $\sum_{i=1}^{I} \zeta_{i j} v_{i j}$ that the objective of the manager can be rewritten as

$$
\phi_{j}=\pi_{j}+\sum_{k \neq j}^{n} \lambda_{j k} \pi_{k} \text {, where } \lambda_{j k} \equiv \frac{\sum_{i=1}^{I} \zeta_{i j} v_{i k}}{\sum_{i=1}^{I} \zeta_{i j} v_{i j}} .
$$

The parameter $\lambda_{j k}$ is the relative weight that the manager of firm $j$ places on the profit of firm $k$ in relation to the own profit (of firm $j$ ) and reflects the control of firm $j$ by investors with financial interests in firms $j$ and $k$. For the manager of firm $j$ to put weight on the interest of investor $i$ we need $\zeta_{i j} v_{i k}>0$ : investor $i$ has to have a stake in firm $k\left(v_{i k}>0\right)$ and some control over firm $j\left(\zeta_{i j}>0\right)$. The weight $\lambda_{j k}$ is larger the more firm $j$ is controlled (high $\zeta_{i j}$ ) by investors with high stakes in firm $k$ (high $v_{i k}$ ) and the less concentrated the ownership and control of firm $j$ (low denominator $\sum_{i=1}^{I} \zeta_{i j} v_{i j}$ ). The numerator $\sum_{i=1}^{I} \zeta_{i j} v_{i k}$ is a measure of the ownership concentration and control of firm $k$. As the ratio $\lambda_{j k}$ increases, the influence of the common owners of firm $k$ over the manager of firm $j$ increases.

We next discuss the cases of silent financial interests and proportional control. In both cases we assume that each firm has a reference shareholder and each investor acquires a share $\alpha$ of the firms which are not under his control. The reference shareholder keeps an interest $1-(I-1) \alpha$ in his firm and we assume that $\alpha I<1$ so that $1-(I-1) \alpha>\alpha$.

Silent Financial Interest (SFI). In this case, each owner (i.e., the majority or dominant shareholder) $i$ retains full control of the acquiring firm and is entitled to a share $\alpha$ of the acquired firms' profits-but exerts no influence over the latter's decisions. Then $\lambda^{\mathrm{SFI}}=\alpha /[1-(I-1) \alpha]$ is just the ratio of the share on an acquired firm $k\left(v_{i k}=\alpha\right.$ in $k$, numerator of $\left.\lambda_{j k}\right)$ over 
the share in the own firm $j\left(\zeta_{i j} v_{i j}=1-(I-1) \alpha\right.$, denominator of $\left.\lambda_{j k}\right){ }^{1}$ The result is that $\lambda_{j k}$ is increasing in the number of investors $I$ since when $I$ increases investor $i$ has less of a financial interest in his own firm (and when $\alpha$ increases then on a double account $\lambda_{j k}$ increases). The driving force is that $\lambda_{j k}$ increases as the size of the interest of undiversified shareholders diminishes. The upper bound of common-ownership is $\alpha=1 / I$, in which case $\lambda^{\mathrm{SFI}}=1$.

Proportional Control (PC). Under proportional control, the firm's manager accounts for shareholders' own-firm interests in other firms in proportion to their respective stakes $\zeta_{i j}=v_{i j}$. In this case we have that $\lambda_{j k}=\left(\sum_{i=1}^{I} v_{i j} v_{i k}\right) /\left(\sum_{i=1}^{I} v_{i j}{ }^{2}\right)$, where the denominator is the HHI on ownership shares of firm $j$ and under symmetry $\lambda^{\mathrm{PC}}$ equals

$$
\left\{2[1-(I-1) \alpha] \alpha+(I-2) \alpha^{2}\right\} /\left\{[1-(I-1) \alpha]^{2}+(I-1) \alpha^{2}\right\} \cdot{ }^{2}
$$

As with SFI, here $\lambda^{\mathrm{PC}}=1$ when $\alpha=1 / I$. For $\alpha<1 / I$, then $\lambda^{\mathrm{PC}}$ is increasing in both $I$ and $\alpha$. The effects are more complex with proportional control but the relative weight of the profit of $k$ over $j$ ends up being monotone in the number of investors $I$ and $\alpha$. Both the numerator and denominator of $\lambda^{\mathrm{PC}}$ decrease with $I$ but the denominator decreases more indicating that the ownership concentration of the firm's manager decreases by more than the one of other firms when $I$ increases, inducing the manager to put a lower weight on the profits of other firms. The driving force again is the decline in the interest of the undiversified stake of reference investors $1-(I-1) \alpha$ as $I$ or $\alpha$ increase.

Cross-ownership We assume here that each of the $n$ firms may acquire their rivals' stock in the form of investments with no control rights. The profit of firm $j$ is given by $\phi_{j}=$ $\pi_{j}+\sum_{k \neq j} \alpha_{j k} \phi_{k}$, where $\alpha_{j k}$ is the firm $j$ 's ownership stake in firm $k$. One can derive the profit for each firm by denoting $\boldsymbol{\phi} \equiv\left(\phi_{1}, \ldots, \phi_{n}\right)^{\prime}$ and $\boldsymbol{\pi} \equiv\left(\pi_{1}, \ldots, \pi_{n}\right)^{\prime}$, and solving the matrix equation: $\phi=\boldsymbol{\pi}+\mathbf{A} \phi$, where $\mathbf{A}$ is the $n \times n$ matrix with the ownership stakes with 0's in the diagonal and $\alpha_{j k}$ off-diagonal. Thus, $\phi=\boldsymbol{\Theta} \boldsymbol{\pi}$, where $\boldsymbol{\Theta}=(\mathbf{I}-\mathbf{A})^{-1}$ is the inverse of the Leontief matrix; its coefficients $\theta_{j k}$ represent the effective or imputed stake in firm $k$ 's profits received by a "real" equity holder with a $1 \%$ direct stake in firm $j$. We examine the symmetric case: $\alpha_{j k}=\alpha_{k j} \equiv \alpha$ for all $j \neq k$, and $\alpha_{j j}=0$ for all $j$. The formula for the coefficients of matrix $\Theta$ when stakes are

\footnotetext{
${ }^{1}$ If $i$ owns and controls $j$, then (i) $\zeta_{i j}=1$ and $\zeta_{i k}=0$ for $k \neq j ; v_{i j}=1-(I-1) \alpha$ and $v_{i k}=\alpha$ for $k \neq j$, and the manager of firm $j$ maximizes $\sum_{k} v_{i k} \pi_{k}$.

${ }^{2}$ Suppose that each investor acquires a share $\alpha$ of those other firms. To compute $\lambda_{j k}$ for a given $k \neq j$, note that if $i$ is the majority shareholder of $j$ then $\zeta_{i j}=1-(I-1) \alpha$ and $v_{i k}=\alpha$; if $i^{\prime}$ is the majority shareholder of $k$, then $\zeta_{i^{\prime} j}=\alpha$ and $i^{\prime}$ receives an own-firm profit share of $v_{i^{\prime} k}=1-(I-1) \alpha$. Finally, there are $I-2$ investors who are minority shareholders of $j$ and $k$; for these investors, the product of their profit shares (and control) is equal to $\alpha^{2}$. This explains the numerator of $\lambda_{j k}$. The denominator follows similarly and we obtain the expression for $\lambda^{\mathrm{PC}}$.
} 
symmetric is, for $\alpha<1 /(n-1), \theta_{j j}=\frac{1-(n-2) \alpha}{[1-(n-1) \alpha](\alpha+1)}$ and $\theta_{j k}=\frac{\alpha}{[1-(n-1) \alpha](\alpha+1)}$ for all $j$ and all $j \neq k{ }^{3}$ Hence, the profit of firm $j$ with symmetric stakes is given by

$$
\phi_{j}=\frac{1-(n-2) \alpha}{[1-(n-1) \alpha](\alpha+1)} \pi_{j}+\frac{\alpha}{[1-(n-1) \alpha](\alpha+1)} \sum_{k \neq j} \pi_{k}
$$

Maximizing the above expression is equivalent to maximizing $\pi_{j}+\lambda \sum_{k \neq j} \pi_{k}$, where $\lambda=\lambda^{\mathrm{CO}} \equiv$ $\alpha /[1-(n-2) \alpha]$.

Comparative statics. The results for $\lambda^{S F I}$ and $\lambda^{C O}$ follow by inspection. Regarding the case of proportional control, we have that

$$
\frac{\partial \lambda^{P C}}{\partial I}=\frac{\alpha^{2}\left[\alpha^{2} I^{2}-4 \alpha I+3\right]}{\left(\alpha^{2} I^{2}-\alpha^{2} I-2 \alpha I+2 \alpha+1\right)^{2}} ; \quad \frac{\partial \lambda^{P C}}{\partial \alpha}=\frac{2(1-\alpha I)}{\left(\alpha^{2} I^{2}-\alpha^{2} I-2 \alpha I+2 \alpha+1\right)^{2}} .
$$

Therefore, $\partial \lambda^{P C} / \partial I>0$ iff $\rho_{P C}(\alpha)=\alpha^{2}\left(\alpha^{2} I^{2}-4 \alpha I+3\right)>0$ for any $I \geq 2$ and $\alpha<1 / I$. Solving for $\rho_{P C}(\alpha)=0$, the quadratic $\left(\alpha^{2} I^{2}-4 \alpha I+3\right)$ gives the solutions $\alpha=1 / I$ and $\alpha=3 / I$. For $\alpha \in(0,1 / I),\left(\alpha^{2} I^{2}-4 \alpha I+3\right)>0$ and $\alpha^{2}>0$ and, thus, $\rho_{P C}(\alpha)>0$.

By differentiating with respect to $\alpha$, we obtain $\rho_{P C}^{\prime}=4 \alpha\left(\alpha^{2} I^{2}-3 \alpha I+3 / 2\right)>0$ for $\alpha \in$ $(0,1 / I)$. Therefore, $\rho_{P C}>0$ for $\alpha \in(0,1 / I)$ since $\rho_{P C}(0)=0$.

Clearly, $\partial \lambda^{P C} / \partial \alpha>0$ for $\alpha<1 / I$.

Ranking. Let us compare $\lambda^{S F I}$ and $\lambda^{P C}$; after simplifying we obtain

$$
\lambda^{S F I}-\lambda^{P C}=\frac{\alpha(1-\alpha I)}{-[1-\alpha(I-1)]\left[1+I(I-1) \alpha^{2}-2(I-1) \alpha\right]} .
$$

For $\alpha<1 / I$, we have $\lambda^{S F I}<\lambda^{P C} \quad$ iff $\quad \rho_{S P}(\alpha)=1+I(I-1) \alpha^{2}-2(I-1) \alpha>0$. Note that $\rho_{S P}(0)=1>0$, furthermore $\rho_{S P}^{\prime}(\alpha)=2 I(I-1) \alpha-2(I-1)=2(I-1)(I \alpha-1)<0$. Since $\rho_{S P}^{\prime \prime}(\alpha)=2(I-1) I>0$, the global minimum is located at $\alpha=1 / I$, at which $\rho_{S P}(1 / I)=1 / I>0$. Thus, $\rho_{S P}(\alpha)>0$ and as a result $\lambda^{S F I}<\lambda^{P C}$.

Finally, for $n=I$

$$
\lambda^{S F I}-\lambda^{C O}=\frac{\alpha^{2}}{[-1+(I-2) \alpha][-1+(I-1) \alpha]},
$$

thus $\lambda^{S F I}-\lambda^{C O}>0$ for $\alpha<1 / I$, hence $\lambda^{P C}>\lambda^{S F I}>\lambda^{C O}$. 
Table 4: Summary of Basic Expressions at the Symmetric Equilibrium of the Simultaneous Game

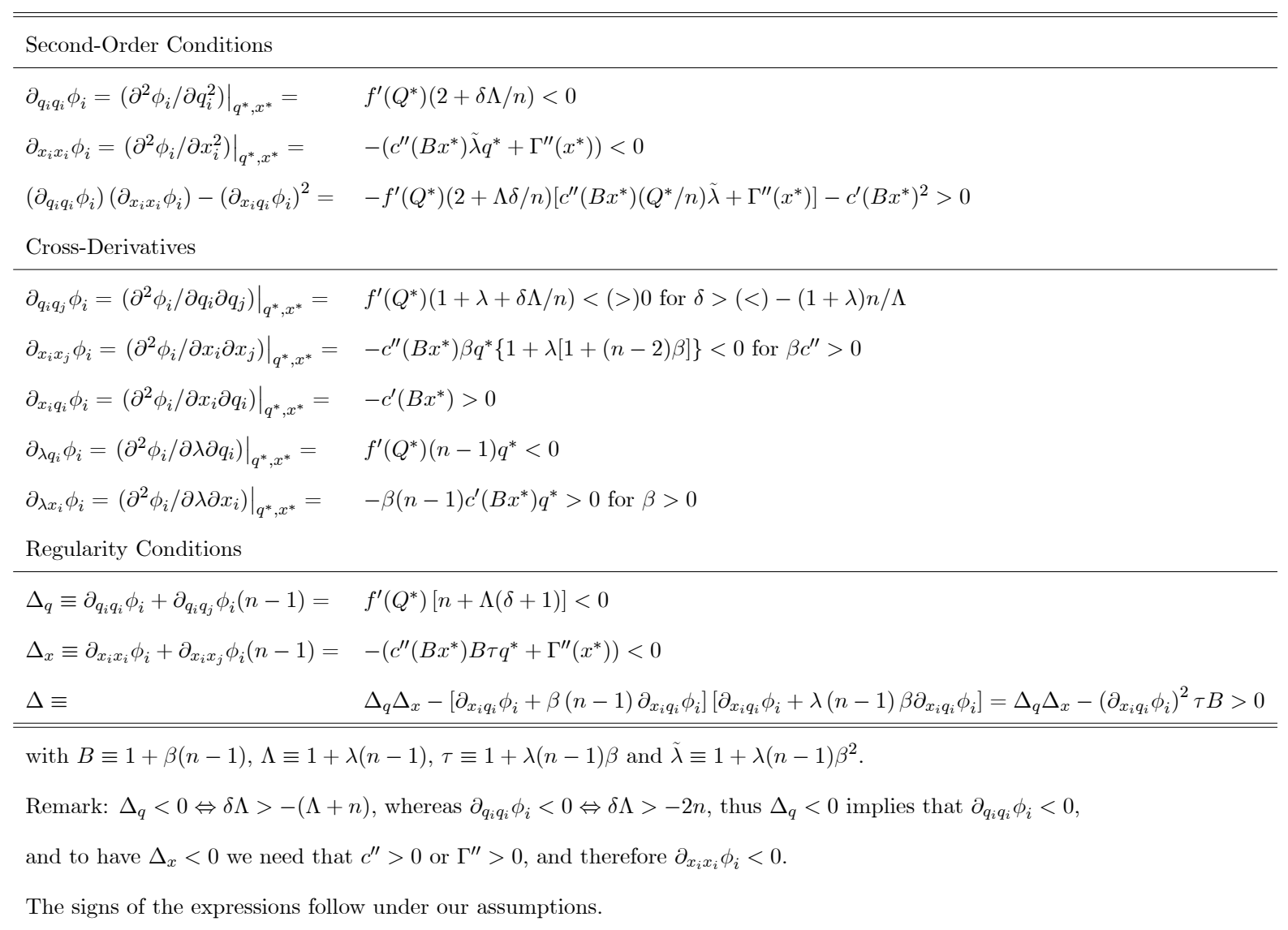

\section{A.1.2 Simultaneous model}

Second order and regularity conditions. To start with, note that

$$
\Delta\left(Q^{*}, x^{*}\right)=-\left[c^{\prime \prime}\left(B x^{*}\right) B \tau\left(Q^{*} / n\right)+\Gamma^{\prime \prime}\left(x^{*}\right)\right]\left[f^{\prime}\left(Q^{*}\right)(\Lambda(1+\delta)+n)\right]-\left(c^{\prime}\left(B x^{*}\right)\right)^{2} \tau B>0 .
$$

In particular, the above condition can be rewritten as $[\Lambda(1+\delta)+n] H(\beta)-\tau B>0$. Second order conditions are: (i) $\partial_{q_{i} q_{i}} \phi_{i}<0$, since $\partial_{q_{i} q_{i}} \phi_{i}=2 f^{\prime}(Q)+\Lambda(Q / n) f^{\prime \prime}(Q)=f^{\prime}(Q)(2+\Lambda \delta / n)$, we have $\partial_{q_{i} q_{i}} \phi_{i}<0$ if $\delta>-2 n / \Lambda$, which is implied by assumption $\Delta_{q}<0$; (ii) $\partial_{x_{i} x_{i}} \phi_{i}<0$, which is trivially satisfied by Assumptions A.2 and A.3; and (iii) $\partial_{q_{i} q_{i}} \phi_{i}\left(\partial_{x_{i} x_{i}} \phi_{i}\right)-\left(\partial_{q_{i} x_{i}} \phi_{i}\right)^{2}>0$, which is equivalent to

$$
c^{\prime}\left(B x^{*}\right)^{2}+f^{\prime}\left(Q^{*}\right)(2+\Lambda \delta / n)\left[c^{\prime \prime}\left(B x^{*}\right)\left(Q^{*} / n\right) \tilde{\lambda}+\Gamma^{\prime \prime}\left(x^{*}\right)\right]<0,
$$

\footnotetext{
${ }^{3}$ See Vives (1999, pp. 145-147) for a solution of a formally identical problem. Gilo et al. (2006, Lemma 1, p.85) also show that $\theta_{j j} \geq 1$ for all $j$, and $0 \leq \theta_{j k}<\theta_{j j}$ for all $j$ and all $j \neq k$.
} 
where $\tilde{\lambda}=1+\lambda(n-1) \beta^{2}$. Noting that $\partial_{q_{i} q_{j}} \phi_{i}=f^{\prime}\left(Q^{*}\right)(1+\lambda)+f^{\prime \prime}\left(Q^{*}\right) \Lambda q^{*}=f^{\prime}\left(Q^{*}\right)(1+\lambda+$ $\delta \Lambda / n)$, we have that

$$
\Delta_{q} \equiv \partial_{q_{i} q_{i}} \phi_{i}+\partial_{q_{i} q_{j}} \phi_{i}(n-1)=f^{\prime}\left(Q^{*}\right)[n+\Lambda(\delta+1)]<0,
$$

which is satisfied if $\delta>-(n+\Lambda) / \Lambda$. Similarly, noting that $\partial_{x_{i} x_{i}} \phi_{i}=-c^{\prime \prime}\left(B x^{*}\right) \tilde{\lambda} q^{*}-\Gamma^{\prime \prime}\left(x^{*}\right)$ and $\partial_{x_{i} x_{j}} \phi_{i}=-c^{\prime \prime}\left(B x^{*}\right) \beta q^{*}\{1+\lambda[1+(n-2) \beta]\}$, it is straightforward to show that

$$
\Delta_{x} \equiv \partial_{x_{i} x_{i}} \phi_{i}+\partial_{x_{i} x_{j}} \phi_{i}(n-1)=-\left[c^{\prime \prime}\left(B x^{*}\right) B \tau q^{*}+\Gamma^{\prime \prime}\left(x^{*}\right)\right]<0,
$$

which is satisfied by Assumptions A.2 and A.3.

Proof of Lemma 1. Using equation (6) and Table 4 we obtain

$$
\frac{\partial x^{*}}{\partial \lambda}=\frac{c^{\prime}\left(B x^{*}\right) f^{\prime}\left(Q^{*}\right)(n-1) q^{*}}{\Delta}\{\beta[\Lambda(1+\delta)+n]-\tau\}
$$

Since $\Delta>0$,

$$
\begin{aligned}
\operatorname{sign}\left\{\frac{\partial x^{*}}{\partial \lambda}\right\} & =\operatorname{sign}\{\beta[\Lambda(1+\delta)+n]-\tau\} \\
& =\operatorname{sign}\left\{\beta-\frac{\tau}{\Lambda(1+\delta)+n}\right\}=\operatorname{sign}\left\{\beta-P^{\prime}(c) \frac{\tau}{n}\right\},
\end{aligned}
$$

where $P^{\prime}(c)=n /[\Lambda(1+\delta)+n]$. Note that $\Lambda(1+\delta)+n>0$ since $\Delta_{q}<0$. Finally, by substituting

$$
\operatorname{sign}\{\beta[\Lambda(1+\delta)+n]-\tau\}=\operatorname{sign}\{\beta(1+n+\delta \Lambda)-1\}
$$

Proof of Corollary 1. From Lemma 1 we have that if $\delta \leq-(1+n) / \Lambda$, so $1+n+\delta \Lambda \leq 0$, then $\partial x^{*} / \partial \lambda<0$, which, using equation (7), in turn implies that $\partial q^{*} / \partial \lambda<0$ : for all $\beta$ only $R_{\mathrm{I}}$ exists. If $\delta>-(n+1) / \Lambda$, then in addition to $R_{\mathrm{I}}$, region $R_{\mathrm{II}}$ exists only if $\delta>-n / \Lambda$ also holds. The reason is that when $1+n+\delta \Lambda>0$, then, from Lemma $1, \partial x^{*} / \partial \lambda>0$ requires that $\beta>1 /(1+n+\delta \Lambda)$. However, $1 /(1+n+\delta \Lambda)<1$ only if $\delta>-n / \Lambda$, in which case there exists some region of feasible spillover values for which $\partial x^{*} / \partial \lambda>0$. Note that for a given $n$, the condition $\delta>-n / \Lambda$ is stricter than the condition $\delta>-(n+1) / \Lambda$. Thus, for $\delta \leq-n / \Lambda$ only $R_{\mathrm{I}}$ exists, and since $-n / \Lambda$ increases with $\lambda$, the result holds for any $\lambda$ if $\delta \leq-n$. 


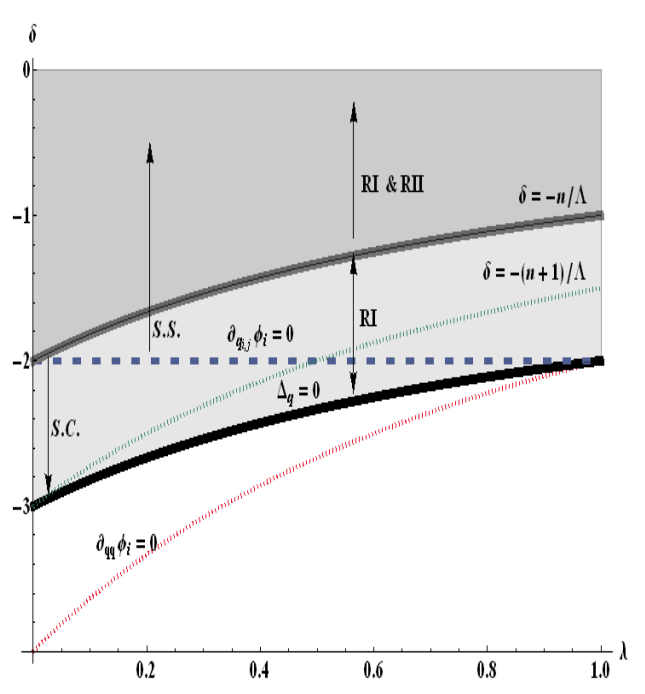

Fig. 5a. $n=2$.

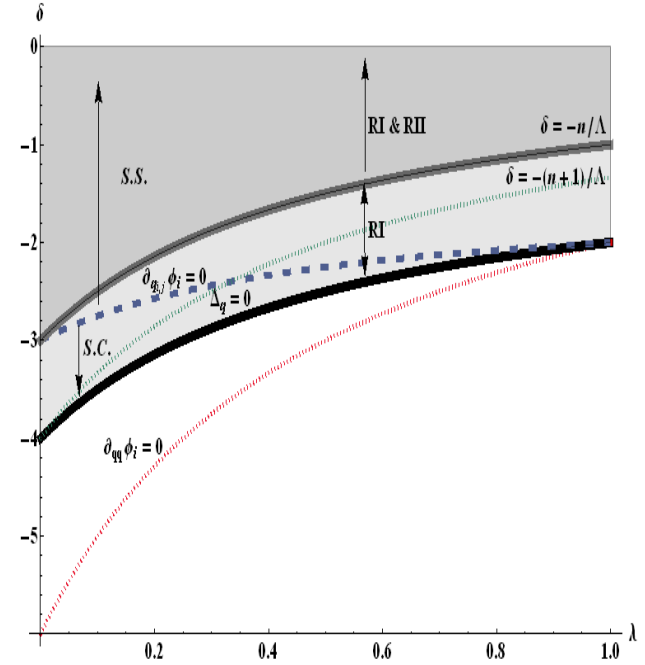

Fig. 5b. $n=3$.

Fig. 5. Existence of regions $R_{\mathrm{I}}$ and $R_{\mathrm{II}}$ with second-order, stability and strategic complements/substitutes output competition conditions.

Proof of Lemma 2. If we totally differentiate the first order conditions (FOCs) and solve for $\partial q^{*} / \partial \lambda$, we obtain

$$
\frac{\partial q^{*}}{\partial \lambda}=\frac{(n-1)\left(Q^{*} / n\right)}{\Delta} \beta c^{\prime}\left(B x^{*}\right)^{2}\left\{B+\frac{f^{\prime}\left(Q^{*}\right)}{\beta c^{\prime}\left(B x^{*}\right)^{2}}\left[c^{\prime \prime}\left(B x^{*}\right)\left(Q^{*} / n\right) B \tau+\Gamma^{\prime \prime}\left(x^{*}\right)\right]\right\} .
$$

Let $H \equiv \beta\left(\partial_{\lambda q_{i}} \phi_{i} / \partial_{\lambda x_{i}} \phi_{i}\right)\left(\Delta_{x} / \partial_{x_{i} q_{i}} \phi_{i}\right)=-\left(f^{\prime}\left(Q^{*}\right) / c^{\prime}\left(B x^{*}\right)^{2}\right)\left[c^{\prime \prime}\left(B x^{*}\right)\left(Q^{*} / n\right) B \tau+\Gamma^{\prime \prime}\left(x^{*}\right)\right]$, evaluated at the equilibrium $\left(Q^{*}, x^{*}\right)$. From the requirement that $c^{\prime \prime}>0$ or $\Gamma^{\prime \prime}>0$ we obtain that $\lim _{\beta \rightarrow 0} H / \beta=\infty$. $H$ is continuous in $\beta$ as long as $Q^{*}(\beta), x^{*}(\beta)$ are since all the functions involved in the definition of $H$ are continuous and $c^{\prime}<0$. We have that $Q^{*}(\beta), x^{*}(\beta)$ are in fact differentiable given our assumptions (see the proof of Proposition 3). The above expression can be rewritten as

$$
\frac{\partial q^{*}}{\partial \lambda}=\frac{(n-1)\left(Q^{*} / n\right)}{\Delta} \beta\left(c^{\prime}\left(B x^{*}\right)\right)^{2}\left(B-\frac{H}{\beta}\right),
$$

thus sign $\left\{\partial q^{*} / \partial \lambda\right\}=\operatorname{sign}\{\beta B-H\}$

Proof of Corollary 2. Under A.4 and Lemma $2, \partial q^{*} / \partial \lambda>0$ (so $R_{\text {III }}$ exists) if $\beta>\beta^{\prime}$. We now show that the condition $n>H(1)$ guarantees that $\beta^{\prime}<1$. First, note that $\lim _{\beta \rightarrow 0} H / \beta=\infty$ (when $c^{\prime \prime}>0$ or $\Gamma^{\prime \prime}>0$ ), while $B=1$ at $\beta=0$. Since $H(\beta) / \beta$ is downward sloping, by continuity there exists only one value for $\beta\left(=\beta^{\prime}\right)$ at which $H(\beta)=\beta B$. If the condition $H(\beta)<\beta B$ holds at $\beta=1$ (which is equivalent to the condition $n>H(1)$ ), then necessarily $H / \beta$ intersects $B$ at some $\beta$ less than 1 , thus $\beta^{\prime}<1$. 
Proof of Proposition 2. Profit per firm as a function of $\lambda$ at equilibrium is given by

$$
\pi^{*}(\lambda)=\left(f\left(Q^{*}\right)-c\left(B x^{*}\right)\right) q^{*}-\Gamma\left(x^{*}\right)
$$

By differentiating $\pi^{*}$ with respect to $\lambda$, we obtain

$$
\pi^{* \prime}(\lambda)=f^{\prime}\left(Q^{*}\right) n \frac{\partial q^{*}}{\partial \lambda} q^{*}-c^{\prime}\left(B x^{*}\right) B \frac{\partial x^{*}}{\partial \lambda} q^{*}+\left(f\left(Q^{*}\right)-c\left(B x^{*}\right)\right) \frac{\partial q^{*}}{\partial \lambda}-\Gamma^{\prime}\left(x^{*}\right) \frac{\partial x^{*}}{\partial \lambda} .
$$

Using that in equilibrium $f\left(Q^{*}\right)-c\left(B x^{*}\right)=-f^{\prime}\left(Q^{*}\right) \Lambda q^{*}$ and $\Gamma^{\prime}\left(x^{*}\right)=-c^{\prime}\left(B x^{*}\right) q^{*} \tau$, we can rewrite the above expression as

$$
\begin{aligned}
\pi^{* \prime}(\lambda) & =f^{\prime}\left(Q^{*}\right) n \frac{\partial q^{*}}{\partial \lambda} q^{*}-c^{\prime}\left(B x^{*}\right) B \frac{\partial x^{*}}{\partial \lambda} q^{*}-f^{\prime}\left(Q^{*}\right) \Lambda q^{*} \frac{\partial q^{*}}{\partial \lambda}+c^{\prime}\left(B x^{*}\right) q^{*} \tau \frac{\partial x^{*}}{\partial \lambda} \\
& =f^{\prime}\left(Q^{*}\right)(n-\Lambda) q^{*} \frac{\partial q^{*}}{\partial \lambda}+c^{\prime}\left(B x^{*}\right)(\tau-B) q^{*} \frac{\partial x^{*}}{\partial \lambda} \\
& =(n-1)(1-\lambda) q^{*}\left(f^{\prime}\left(Q^{*}\right) \frac{\partial q^{*}}{\partial \lambda}-\beta c^{\prime}\left(B x^{*}\right) \frac{\partial x^{*}}{\partial \lambda}\right) .
\end{aligned}
$$

In $R_{\mathrm{II}}$, we have that $\partial x^{*} / \partial \lambda>0$ and $\partial q^{*} / \partial \lambda<0$. Hence from the above expression it is clear that $\pi^{* \prime}(\lambda)>0$. Note also that when $\beta=0$, the equilibrium is in $R_{\mathrm{I}}$, and therefore $\pi^{* \prime}(\lambda)>0$ since $\partial q^{*} / \partial \lambda<0$. To determine $\operatorname{sign}\left\{\pi^{* \prime}(\lambda)\right\}$ in $R_{\mathrm{I}}$ and $R_{\mathrm{III}}$ for $\beta>0$, we replace $\partial q^{*} / \partial \lambda$ and $\partial x^{*} / \partial \lambda$ with the expressions derived in the proofs of Lemmata 1, 2:

$$
\begin{aligned}
\pi^{* \prime}(\lambda)= & (n-1)(1-\lambda) q^{*}\left(f^{\prime}\left(Q^{*}\right) \frac{(n-1) q^{*}}{\Delta} c^{\prime}\left(B x^{*}\right)^{2} \beta\left(B-\frac{H(\beta)}{\beta}\right)\right. \\
& \left.-\beta c^{\prime}\left(B x^{*}\right) \frac{(n-1) q^{*}}{\Delta} f^{\prime}\left(Q^{*}\right) c^{\prime}\left(B x^{*}\right)\{\beta[\Lambda(1+\delta)+n]-\tau\}\right) \\
= & \vartheta_{\pi}\left\{\beta[\Lambda(1+\delta)+n]-\tau+\frac{H(\beta)}{\beta}-B\right\},
\end{aligned}
$$

where $\vartheta_{\pi} \equiv(n-1)(1-\lambda) q^{*}\left[(n-1) q^{*} / \Delta\right] c^{\prime}\left(B x^{*}\right)^{2} \beta\left(-f^{\prime}\left(Q^{*}\right)\right)$ is positive. Therefore,

$$
\operatorname{sign}\left\{\pi^{* \prime}(\lambda)\right\}=\operatorname{sign}\left\{(n+1+\delta \Lambda) \beta-1+\frac{H(\beta)}{\beta}-B\right\} \text {, }
$$

so it follows that $\pi^{* \prime}(\lambda)>0$ if

$$
\begin{gathered}
1-(n+1+\delta \Lambda) \beta<\frac{H(\beta)}{\beta}-B, \text { or equivalently } \\
2(1-\beta)-\delta \Lambda \beta<\frac{H(\beta)}{\beta} .
\end{gathered}
$$

From Table 4 and using that in equilibrium $\tau q^{*}=-\Gamma^{\prime}\left(x^{*}\right) / c^{\prime}\left(B x^{*}\right)$, the regularity condition 
can be written as

$$
-\left(-c^{\prime \prime}\left(B x^{*}\right) B \frac{\Gamma^{\prime}\left(x^{*}\right)}{c^{\prime}\left(B x^{*}\right)}+\Gamma^{\prime \prime}\left(x^{*}\right)\right) \frac{f^{\prime}\left(Q^{*}\right)}{c^{\prime}\left(B x^{*}\right)^{2}}[\Lambda(1+\delta)+n]-\tau B>0 .
$$

Noting that

$$
H(\beta)=\frac{-f^{\prime}\left(Q^{*}\right)}{c^{\prime}\left(B x^{*}\right)^{2}}\left(-\frac{c^{\prime \prime}\left(B x^{*}\right)}{c^{\prime}\left(B x^{*}\right)} B \Gamma^{\prime}\left(x^{*}\right)+\Gamma^{\prime \prime}\left(x^{*}\right)\right),
$$

we can rewrite the regularity condition in terms of $H$ as follows: $[\Lambda(1+\delta)+n] H(\beta)-\tau B>0$, with $\Lambda(1+\delta)+n>0$ since $\Delta_{q}<0$. Thus, if the equilibrium is regular:

$$
\frac{H(\beta)}{\beta}>\frac{\tau B}{[\Lambda(1+\delta)+n] \beta} .
$$

Then, we only have to show that:

$$
\tilde{g}(\beta) \equiv \tau B>\tilde{h}(\beta) \equiv[2(1-\beta)-\Lambda \delta \beta][\Lambda(1+\delta)+n] \beta
$$

holds. Note that $\tilde{g}(0)=1, \tilde{g}^{\prime}(\beta)>0, \tilde{g}^{\prime \prime}(\beta)>0$ for $\beta>0$ and $\tilde{g}^{\prime \prime}(0)=0$. On the other hand, $\tilde{h}(0)=0$ and

$$
\tilde{h}^{\prime}(\beta)=2[\Lambda(1+\delta)+n][1-(2+\Lambda \delta) \beta]
$$

Furthermore, it can be shown that solving the equation $\tilde{g}(\beta)=\tilde{h}(\beta)$ for $\beta$ yields the following two roots:

$$
\beta_{1}=\frac{1}{\Lambda \delta+n+1} \text { and } \beta_{2}=\frac{1}{\Lambda(\delta+1)+1} .
$$

Consider $R_{\mathrm{I}}$. If the smallest (positive) root in this region is larger or equal to the spillover threshold $\underline{\beta}$ that determines $R_{\mathrm{I}}$ (i.e. for $\beta<\underline{\beta}, \frac{\partial x^{*}}{\partial \lambda}<0$ for all $\lambda$ ), then $\tilde{g}(\beta)>\tilde{h}(\beta)$ in $R_{\mathrm{I}}$, and consequently, $\pi^{* \prime}(\lambda)>0$. First, note that when $\Lambda \delta+n+1>0, \beta_{1}=\underline{\beta}$. We distinguish the following cases:

- If $\Lambda \delta+n+1>0$, then: if $\Lambda(\delta+1)+1>0$, (for $\lambda<1) \beta_{2}>\beta_{1}=\underline{\beta}>0$, while if $\Lambda(\delta+1)+1<0, \beta_{1}=\underline{\beta}>0>\beta_{2}$, so in any case $\beta_{1}=\underline{\beta}$ is the smallest positive root in the region and, thus, $\tilde{g}(\beta)>\tilde{h}(\beta)$ for $\beta \in\left(0, \beta_{1}\right)$. Also, in any case for $\beta=\beta_{1}$, $\partial x^{*} / \partial \lambda=0$ and so $\operatorname{sign}\left\{\pi^{* \prime}(\lambda)\right\}=\operatorname{sign}\left\{f^{\prime}\left(Q^{*}\right) \partial q^{*} / \partial \lambda\right\}$, which is positive in $R_{\mathrm{I}}$ since in this region: $\partial q^{*} / \partial \lambda<0$.

- If $\Lambda \delta+n+1<0$, then $0>\beta_{1}>\beta_{2}$ (for $\left.\lambda<1\right)$ and $\tilde{h}^{\prime \prime}(\beta)>0$, so $\tilde{g}(\beta)>\tilde{h}(\beta)$ for all $\beta$.

Now consider $R_{\mathrm{III}}$, which may exist only if $\delta>-n / \Lambda$, in which case $\beta_{1}>0$. Furthermore, $\beta^{\prime} \geq \beta_{1}$. We show that for any $\beta>\beta^{\prime}, \tilde{g}(\beta)>\tilde{h}(\beta)$. We distinguish the following cases: 
- If $\delta>-2 / \Lambda$, then $\beta_{2}>\beta_{1}>0$ (for $\lambda<1$ ) and $\tilde{h}^{\prime \prime}(\beta)<0$. Hence, $\tilde{g}(\beta)>\tilde{h}(\beta)$ for $\beta>\beta_{2}$. Thus, it suffices to show that $\beta^{\prime}>\beta_{2}$. Note that if $\pi^{\prime}(\lambda)>0$ for $\beta=\beta^{\prime}$, then necessarily $\beta^{\prime}>\beta_{2}$ since $\beta^{\prime}>\beta_{1}$ and $\pi^{\prime}(\lambda)<0$ for $\beta \in\left(\beta_{1}, \beta_{2}\right)$. Since condition (24) holds at $\beta=\beta^{\prime}$ : $H\left(\beta^{\prime}\right) / \beta^{\prime}-\left[1+\beta^{\prime}(n-1)\right]=0>1-(n+1+\delta \Lambda) \beta$, we have $\beta^{\prime}>\beta_{2}$.

- If $-(\Lambda+1) / \Lambda<-n / \lambda<\delta<-2 / \Lambda$ or $-n / \lambda<-(\Lambda+1) / \Lambda<\delta<-2 / \Lambda$, then $\beta_{2}>\beta_{1}>0$ (for $\lambda<1$ ) and $\tilde{h}^{\prime \prime}(\beta)>0$, so we can conclude that $\beta^{\prime}>\beta_{2}$.

- If $-n / \lambda<\delta<-(\Lambda+1) / \Lambda<-2 / \Lambda$, then $\beta_{1}>0, \beta_{2}<0$ and $\tilde{h}^{\prime \prime}(\beta)>0$, so $\tilde{g}(\beta)>\tilde{h}(\beta)$ only for $\beta<\beta_{1}$. Also, for $\beta^{\prime}<1$, condition (24) holds, so $\tilde{g}(\beta)>\tilde{h}(\beta)$ for $\beta \geq \beta^{\prime}$. But then it should be $\beta^{\prime}<\beta_{1}$, a contradiction, so in this case $R_{I I I}$ does not exist.

Proof of Proposition 3. By totally differentiating the two FOCs with respect to $\beta$, we obtain

$$
\begin{aligned}
& \frac{\partial q^{*}}{\partial \beta}=\frac{1}{\Delta}\left[\left(\partial_{\beta x_{i}} \phi_{i}\right)\left(\partial_{x_{i} q_{i}} \phi_{i}\right) B-\left(\partial_{\beta q_{i}} \phi_{i}\right) \Delta_{x}\right] \\
& \frac{\partial x^{*}}{\partial \beta}=\frac{1}{\Delta}\left[\left(\partial_{\beta q_{i}} \phi_{i}\right)\left(\partial_{x_{i} q_{i}} \phi_{i}\right) \tau-\left(\partial_{\beta x_{i}} \phi_{i}\right) \Delta_{q}\right] .
\end{aligned}
$$

Since $\partial_{x_{i} q_{i}} \phi_{i}>0$ and $\partial_{\beta q_{i}} \phi_{i}>0, \Delta>0, \Delta_{x}<0$ and $\Delta_{q}<0$, the sign of the impact of $\beta$ on output and R\&D in equilibrium depends on the sign of $\partial_{\beta x_{i}} \phi_{i}$. It can be shown that

$$
\partial_{\beta x_{i}} \phi_{i}=-c^{\prime}\left(B x^{*}\right) \frac{(n-1) q^{*}}{B} \tau\left(\frac{\lambda B}{\tau}-\chi\left(B x^{*}\right)\right)
$$

and the result follows.

Proof of Proposition 4. To prove Proposition 4 a few preliminary lemmata (assuming A.1-A.4) are useful.

LEMMA 5 Suppose that $\delta>-2$, then for given $\lambda, W^{\prime}(\lambda)>0$ iff $\beta>\hat{\beta}(\lambda)$ where $\hat{\beta}$ is the unique positive solution to the equation

$$
\frac{H(\beta)}{\beta}-B=[(n-\Lambda) / \Lambda][(1+n+\delta \Lambda) \beta-1]
$$

Proof. We first derive the condition that determines $\hat{\beta}$. By inserting $\partial q^{*} / \partial \lambda$ and $\partial x^{*} / \partial \lambda$ 
(given in proofs of Lemmata 1 and 2) into (9) we obtain:

$$
\begin{aligned}
W^{\prime}(\lambda)= & -\Lambda f^{\prime}\left(Q^{*}\right) \frac{(n-1) q^{*}}{\Delta} c^{\prime}\left(B x^{*}\right)^{2} \beta\left(B-\frac{H(\beta)}{\beta}\right) Q^{*} \\
& -(1-\lambda) \beta(n-1) c^{\prime}\left(B x^{*}\right) \frac{(n-1) q^{*}}{\Delta} f^{\prime}\left(Q^{*}\right) c^{\prime}\left(B x^{*}\right)\{\beta[\Lambda(1+\delta)+n]-\tau\} Q^{*}, \\
= & \vartheta_{w}\left(\Lambda\left(B-\frac{H(\beta)}{\beta}\right)+(1-\lambda)(n-1)\{\beta[\Lambda(1+\delta)+n]-\tau\}\right),
\end{aligned}
$$

where $\vartheta_{w} \equiv\left[(n-1) q^{*} / \Delta\right] c^{\prime}\left(B x^{*}\right)^{2}\left(-f^{\prime}\left(Q^{*}\right)\right) \beta Q^{*}$ is positive. Note that $(1-\lambda)(n-1)=n-\Lambda$, thus for $\beta>0, W^{\prime}(\lambda)>0$ iff

$$
\frac{H(\beta)}{\beta}-B<\frac{n-\Lambda}{\Lambda}[(1+n+\delta \Lambda) \beta-1]
$$

Note that $\lim _{\beta \rightarrow 0} H / \beta=\infty$ and (by Assumption A.4) the left-hand side of (30) is decreasing in $\beta$. The right-hand side of (30) is increasing in $\beta$ (since $1+n+\delta \Lambda>0$ holds when $R_{\mathrm{II}}$ and $R_{\text {III }}$ exist) and finite at $\beta=0$. Thus, there exists a unique positive threshold $\hat{\beta}$ that solves the equation (29), and for any $\beta>\hat{\beta}$ condition (30) holds, that is, $W^{\prime}(\lambda)>0$

LEMMA 6 We have that $\hat{\beta}(\lambda)<\beta^{\prime}(\lambda)$ for all $\lambda$, which implies that $\bar{\beta} \equiv \hat{\beta}(0)<\beta^{\prime}(0)$. Furthermore, $\bar{\beta}<1$ if

$$
n+(n-1)(\delta+n)-H(1)>0 .
$$

Proof. We first show that $\beta^{\prime}(\lambda)>\hat{\beta}(\lambda)$ for any $\lambda$, and as a result $\beta^{\prime}(0)>\bar{\beta} \equiv \hat{\beta}(0)$. Suppose that for given $\lambda, \hat{\beta}>\beta^{\prime}$, then from Lemma 2 we have that for $\beta \in\left(\beta^{\prime}, \hat{\beta}\right)$ it holds that $\partial q^{*} / \partial \lambda>0$. Thus, from equation (7) it also holds that $\partial x^{*} / \partial \lambda>0$, which given equation (9) implies that $W^{\prime}(\lambda)>0$. However, we have that $W^{\prime}(\lambda)<0$ for $\beta<\hat{\beta}$, a contradiction. Suppose now that $\hat{\beta}=\beta^{\prime}$, then we can pick $\beta$ such that $\beta=\hat{\beta}=\beta^{\prime}$, and since $H-\left.\beta B\right|_{\beta=\beta^{\prime}}=0$, from equation (29) we have that $\hat{\beta}=\beta^{\prime}=1 /(1+n+\delta \Lambda)$, which implies that $\partial x^{*} / \partial \lambda=0$ (see proof of Lemma 1), and from equation (7) this in turn implies that $\partial q^{*} / \partial \lambda<0$. However, at $\beta=\beta^{\prime}$, $\beta B-H=0$, so $\partial q^{*} / \partial \lambda=0$, a contradiction.

The proof of Lemma 5 shows that $W^{\prime}(\lambda)>0$ for some $\lambda$ if $\beta>\hat{\beta}(\lambda)$, where $\hat{\beta}$ is the unique positive solution to the equation (29). Furthermore, $\hat{\beta}<1$ if condition (30) evaluated at $\beta=1$ holds. Therefore, by evaluating (30) at $\lambda=0$ and $\beta=1$ we obtain that condition (31) ensures that $\bar{\beta}<1$.

We turn now to prove successively each of the statements of Proposition 4 . Let $\delta>-2$ :

i) $\lambda_{\mathrm{TS}}^{o}=\lambda_{\mathrm{CS}}^{o}=0$ if $\beta \leq \bar{\beta}$. First, we show that there does not exist $\beta<\bar{\beta}$ such that $W^{\prime}(\lambda)>0$ for some positive $\lambda$. This follows trivially from the assumption that $W(\lambda)$ is single 
peaked: since for any $\beta \leq \bar{\beta}, W^{\prime}(0) \leq 0$, we have that $W^{\prime}(\lambda)<0$ for all positive $\lambda$, otherwise there would exist another stationary point that is a (local) minimum, a contradiction. In addition, if $\beta \leq \bar{\beta}$, then $\lambda_{\mathrm{CS}}^{o}=0$ : from Lemma 6 we know that $\beta^{\prime}(\lambda)>\bar{\beta}=\hat{\beta}(0)$ for all $\lambda$. For $\beta \leq \bar{\beta}$ we have then that $C S^{\prime}(\lambda)<0$ for all $\lambda$, thus $\lambda_{\mathrm{CS}}^{o}=0$.

ii) $\lambda_{\mathrm{TS}}^{o}>\lambda_{\mathrm{CS}}^{o}=0$ if $\beta \in\left(\bar{\beta}, \beta^{\prime}(0)\right)$. Since $\bar{\beta}=\hat{\beta}(0)$, the result that $\lambda_{\mathrm{TS}}^{o}>0$ for $\beta>\bar{\beta}$ follows immediately from Lemma 5 because then $W^{\prime}(0)>0$. In addition, $\beta<\beta^{\prime}(0)$ yields $\lambda_{\mathrm{CS}}^{o}=0$ : when $H$ is weakly increasing in $\lambda, \beta^{\prime}(\lambda)$ also is, and consequently if $\beta<\beta^{\prime}(0)$, then $\beta<\beta^{\prime}(\lambda)$ for all $\lambda$, i.e., $\partial q^{*} / \partial \lambda<0$ for all $\lambda$, thus $\lambda_{\mathrm{CS}}^{o}=0$.

iii) We first show that $\lambda_{\mathrm{TS}}^{o}>0$ and $\lambda_{\mathrm{CS}}^{o}>0$ if $\beta>\beta^{\prime}(0)$. From Lemma 6 it follows that $\beta>\beta^{\prime}(0)>\bar{\beta}$, which yields $\lambda_{\text {TS }}^{o}>0$. If $\beta>\beta^{\prime}(0)$, Lemma 2 implies that $\partial q^{*} / \partial \lambda>0$ at $\lambda=0$, which implies that $C S^{\prime}(0)>0$, and therefore $\lambda_{\mathrm{CS}}^{o}>0$.

Next we show that $\lambda_{\mathrm{TS}}^{o} \geq \lambda_{\mathrm{CS}}^{o}$ when $H$ is weakly increasing in $\lambda$. Note that $B>H / \beta$ at $\lambda=0$. Since $H$ is weakly increasing in $\lambda$, for a given $\beta$, we may face the following three cases: 1) for all $\lambda, B>H / \beta ; 2)$ there exists an interval (which could be a singleton) $L \subset(0,1]$ at which $H / \beta=B$ but $H / \beta \leq B$ for $\lambda<1 ; 3)$ there exists an interval of values of $\lambda, L \subset(0,1]$ at which $H / \beta=B$ but $H / \beta>B$ for some $\lambda<1$. In all three cases $\lambda_{\mathrm{TS}}^{o} \geq \lambda_{\mathrm{CS}}^{o}$ :

Case 1: Here, $\partial q^{*} / \partial \lambda>0$ and, by (7), $\partial x^{*} / \partial \lambda>0$ for all $\lambda$, which from equation (9) yields $W^{\prime}(\lambda)>0$ for all $\lambda$; thus $\lambda_{\mathrm{TS}}^{o}=\lambda_{\mathrm{CS}}^{o}=1$.

Cases 2 and 3: In these two cases, in the region of values for $\lambda$ where $H / \beta=B$ we have $\partial q^{*} / \partial \lambda=0\left(C S^{\prime}(\lambda)=0\right)$, while $\partial x^{*} / \partial \lambda>0$, consequently $W^{\prime}(\lambda)>0$. It follows that in Case $2, \lambda_{\mathrm{TS}}^{o}=1$, while any $\lambda \in L$ is optimal in terms of CS since $\partial q^{*} / \partial \lambda>0$ for any $\lambda<\min L$, thus $\lambda_{\mathrm{TS}}^{o} \geq \lambda_{\mathrm{CS}}^{o}$; in Case 3, any $\lambda \in L$ is optimal in terms of CS since $\partial q^{*} / \partial \lambda<0$ for $\lambda>\max L$; $\lambda_{\mathrm{TS}}^{o} \geq \max L$ since $W^{\prime}(\lambda)>0$ for lower values of $\lambda$; as a result $\lambda_{\mathrm{TS}}^{o} \geq \lambda_{\mathrm{CS}}^{o}$.

The particular case where $\beta=\beta^{\prime}(0)$ can be dealt with similarly to obtain that $\lambda_{\mathrm{TS}}^{o} \geq \lambda_{\mathrm{CS}}^{o} \geq$ 0 .

Finally, we show that $\lambda_{\mathrm{TS}}^{o}$ and $\lambda_{\mathrm{CS}}^{o}$ are strictly increasing in $\beta$ when $\lambda_{\mathrm{TS}}^{o}$ and $\lambda_{\mathrm{CS}}^{o}$ are in $(0,1)$. We have that

$$
\left.W^{\prime}(\lambda)=-c^{\prime}\left(B x^{*}\right)^{2} f^{\prime}\left(Q^{*}\right)\right) \frac{(n-1) q^{*}}{\Delta} \beta Q^{*} \varphi(\lambda, \beta)
$$

where $\varphi(\lambda, \beta)=\Lambda(B-H / \beta)+(n-\Lambda)(\beta(1+n+\delta \Lambda)-1)$. Consider the FOC of the welfare maximizing problem, $W^{\prime}(\lambda)=0$ if and only if $\varphi(\lambda, \beta)=0$. Given single-peakedness of $W$, $\operatorname{sign}\left\{d \lambda_{\mathrm{TS}}^{o} / d \beta\right\}=\operatorname{sign}\left\{\partial \varphi\left(\lambda_{\mathrm{TS}}^{o}, \beta\right) / \partial \beta\right\}$. We have that

$$
\frac{\partial \varphi}{\partial \beta}=\left[\Lambda \frac{\partial}{\partial \beta}\left(B-\frac{H(\beta)}{\beta}\right)+(n-\Lambda)(1+n+\delta \Lambda)\right]>0
$$


since $H(\beta) / \beta$ is downward sloping, $n-\Lambda \geq 0$, and interior optimal lambdas require that $R_{\mathrm{II}}$ exists, i.e., $\delta>-n / \Lambda$, which in turn implies that $1+n+\delta \Lambda>0$. Similarly, we can show, using the fact that $H(\beta) / \beta$ is decreasing in $\beta$, the result for $\lambda_{\mathrm{CS}}^{o} \in(0,1)$

Proof of Proposition 5. If $\delta>-(1+n) / n$, then $1+n+\delta \Lambda>0$ for all $\lambda$. From Lemma 1 we know that when $\beta \leq 1 /(1+n+\delta \Lambda)$ : $\partial x^{*} / \partial \lambda \leq 0$. From Lemma 5 we have that $W^{\prime}(\lambda)>0$ if $\beta>\hat{\beta}(\lambda)$ where $\hat{\beta}$ is given in Lemma 5. Necessarily, $\hat{\beta}>1 /(1+n+\delta \Lambda)$, otherwise for any $\beta \in$ $[\hat{\beta}, 1 /(1+n+\delta \Lambda)]$, we have that $\partial x^{*} / \partial \lambda \leq 0$, which from equation $(7)$ implies that $\partial q^{*} / \partial \lambda<0$, which using equation (9) yields $W^{\prime}(\lambda)<0$, a contradiction. Since $\hat{\beta}(\lambda)>1 /(1+n+\delta \Lambda)$ for any $\lambda$, then $\hat{\beta}(0)=\bar{\beta}>\underline{\beta}$, and given Lemma $6, \underline{\beta}<\bar{\beta}<\beta^{\prime}(0)$ is established. Next we prove each of the statements. (i) When $\delta>-(1+n) / n$ not only $R_{\mathrm{I}}$ but also $R_{\mathrm{II}}$ may exist for $n \geq 2$ since $\delta>-2$. If $-(1+n) / n<\delta<0$, then $\inf \{1 /(1+n+\Lambda \delta): \lambda \in[0,1]\}=1 /(1+n+\delta)>0$, whereas if $\delta \geq 0, \inf \{1 /(1+n+\Lambda \delta): \lambda \in[0,1]\}=1 /[1+n(1+\delta)]>0$. In both cases, if $\beta \leq \beta$, it follows from Proposition 1 that only $R_{\mathrm{I}}$ can exist. (ii) Lemma 5 ensures that if $\beta>$ $\bar{\beta}=\hat{\beta}(0)$, then $W^{\prime}(0)>0$, thus $\lambda_{\mathrm{TS}}^{o}>0$; (iii) From Lemma 2 we have that if $\beta>\beta^{\prime}(0)$, then $\partial q^{*} /\left.\partial \lambda\right|_{\lambda=0}>0$, which implies that $C S^{\prime}(0)>0: \lambda_{\mathrm{CS}}^{o}>0$.

\section{A.1.3 Two-stage model}

Threshold $\tilde{\beta}(\lambda)$. Let $z_{i}$ be the action of firm $i$ ( $q_{i}$ in Cournot) and let $\mathbf{z}^{*}$ be the $n$-vector of second stage equilibrium actions, then the FOC in the second stage is

$$
\frac{\partial}{\partial z_{i}} \phi_{i}(\cdot)=0
$$

whereas in the first stage is

$$
\frac{\partial}{\partial x_{i}} \phi_{i}\left(\mathbf{z}^{*}(\mathbf{x}), \mathbf{x}, \lambda\right)+\sum_{j \neq i} \frac{\partial}{\partial z_{j}} \phi_{i}\left(\mathbf{z}^{*}(\mathbf{x}), \mathbf{x}, \lambda\right) \frac{\partial}{\partial x_{i}} z_{j}^{*}(\mathbf{x})=0
$$

where $\mathbf{x}$ is the $n$-vector of investment levels. The equilibrium in the two-stage model is thus characterized by the system of equations (32) and (33).

To obtain $\tilde{\beta}(\lambda)$, we first need to obtain the expressions for $\partial z_{i}^{*}(\mathbf{x}) / \partial x_{i}$ and $\partial z_{j}^{*}(\mathbf{x}) / \partial x_{i}$ : we differentiate the FOC (32) with respect to $x_{i}$ and $x_{h}(h \neq i)$, and evaluate both derivatives in the symmetric equilibrium, then

$$
\partial_{z_{i} z_{i}} \phi_{i}(\mathbf{x}) \frac{\partial}{\partial x_{i}} z_{i}^{*}(\mathbf{x})+(n-1) \partial_{z_{i} z_{j}} \phi_{i}(\mathbf{x}) \frac{\partial}{\partial x_{i}} z_{j}^{*}(\mathbf{x})+\partial_{x_{i} z_{i}} \phi_{i}(\mathbf{x})=0
$$


and

$$
\partial_{z_{i} z_{j}} \phi_{i}(\mathbf{x}) \frac{\partial}{\partial x_{i}} z_{i}^{*}(\mathbf{x})+\left[\partial_{z_{i} z_{i}} \phi_{i}(\mathbf{x})+(n-2) \partial_{z_{i} z_{j}} \phi_{i}(\mathbf{x})\right] \frac{\partial}{\partial x_{i}} z_{j}^{*}(\mathbf{x})+\partial_{x_{h} z_{i}} \phi_{i}(\mathbf{x})=0 .
$$

Solving (34) and (35) for $\partial z_{i}^{*}(\mathbf{x}) / \partial x_{i}$ and $\partial z_{j}^{*}(\mathbf{x}) / \partial x_{i}$ and rearranging terms, we obtain:

$$
\frac{\partial}{\partial x_{i}} z_{i}^{*}(\mathbf{x})=\frac{1}{\Omega}\left[\left(-\partial_{x_{i} z_{i}} \phi_{i}\right)\left(\partial_{z_{i} z_{i}} \phi_{i}-\partial_{z_{i} z_{j}} \phi_{i}\right)+(n-1) \partial_{z_{i} z_{j}} \phi_{i}\left(\partial_{x_{h} z_{i}} \phi_{i}-\partial_{x_{i} z_{i}} \phi_{i}\right)\right]
$$

and

$$
\frac{\partial}{\partial x_{i}} z_{j}^{*}(\mathbf{x})=\frac{1}{\Omega}\left(\partial_{x_{i} z_{i}} \phi_{i} \partial_{z_{i} z_{j}} \phi_{i}-\partial_{x_{h} z_{i}} \phi_{i} \partial_{z_{i} z_{i}} \phi_{i}\right)
$$

where

$$
\Omega \equiv\left(\partial_{z_{i} z_{i}} \phi_{i}-\partial_{z_{i} z_{j}} \phi_{i}\right)\left[\partial_{z_{i} z_{i}} \phi_{i}+(n-1) \partial_{z_{i} z_{j}} \phi_{i}\right]
$$

Consider Cournot competition, $z_{i}=q_{i}$. Then, we can rewrite (36) as follows:

$$
\frac{\partial}{\partial x_{i}} q_{j}^{*}(\mathbf{x})=\frac{-c^{\prime}(B x)}{\Omega} \partial_{q_{i} q_{i}} \phi_{i}(\tilde{\beta}(\lambda)-\beta)
$$

where

$$
\tilde{\beta}(\lambda)=\frac{\partial_{q_{i} q_{j}} \phi_{i}}{\partial_{q_{i} q_{i}} \phi_{i}}=\frac{n(1+\lambda)+\Lambda \delta}{2 n+\Lambda \delta}
$$

with $0<\tilde{\beta}(\lambda) \leq 1$.

Proof of Lemma 3. We have that

$$
\tilde{\beta}(\lambda)=\frac{n(1+\lambda)+\Lambda \delta}{2 n+\Lambda \delta} .
$$

By differentiating $\tilde{\beta}$ with respect to $n$ we obtain:

$$
\frac{\partial \tilde{\beta}}{\partial n}=-\frac{\delta(1-\lambda)^{2}}{(2 n+\delta \Lambda)^{2}}
$$

Thus, for $\lambda<1$ and convex demand $(\delta<0), \partial \tilde{\beta} / \partial n>0$; if demand is concave $(\delta>0)$, $\partial \tilde{\beta} / \partial n<0$. Let us now differentiate $\tilde{\beta}$ with respect to $\lambda$ :

$$
\frac{\partial \tilde{\beta}}{\partial \lambda}=\frac{n^{2}(\delta+2)}{(2 n+\delta \Lambda)^{2}}
$$

then, $\partial \tilde{\beta} / \partial \lambda>0$ if $\delta>-2$. Finally, we differentiate $\tilde{\beta}$ with respect to $\delta$ :

$$
\frac{\partial \tilde{\beta}}{\partial \delta}=\frac{\Lambda n(1-\lambda)}{(2 n+\delta \Lambda)^{2}}
$$


Thus, $\partial \tilde{\beta} / \partial \delta>0$ if $\lambda<1$

Proof of Lemma 4. Using (12), by totally differentiating the system formed by $(10 ; 11)$ in a symmetric equilibrium, and solving for $\partial q^{*} / \partial \lambda$ and $\partial x^{*} / \partial \lambda$, we obtain

$$
\begin{aligned}
& \frac{\partial q^{*}}{\partial \lambda}=\frac{1}{\tilde{\Delta}}\left\{\left[\partial_{\lambda x_{i}} \phi_{i}+(n-1) \psi_{\lambda}\right]\left(\partial_{x_{i} q_{i}} \phi_{i}\right) B-\partial_{\lambda q_{i}} \phi_{i}\left[\Delta_{x}+\psi_{x}(n-1)\right]\right\} \\
& \frac{\partial x^{*}}{\partial \lambda}=\frac{1}{\tilde{\Delta}}\left\{\partial_{\lambda q_{i}} \phi_{i}\left[\partial_{x_{i} q_{i}} \phi_{i} \tau+(n-1) \psi_{q}\right]-\left[\partial_{\lambda x_{i}} \phi_{i}+(n-1) \psi_{\lambda}\right] \Delta_{q}\right\},
\end{aligned}
$$

where $\psi_{z} \equiv \partial \psi / \partial z$ with $z=q, x, \lambda$, and

$$
\tilde{\Delta}\left(Q^{*}, x^{*}\right)=\Delta_{q}\left[\Delta_{x}+\psi_{x}(n-1)\right]-\partial_{x_{i} q_{i}} \phi_{i}\left[\partial_{x_{i} q_{i}} \phi_{i} \tau+\psi_{q}(n-1)\right] B
$$

which is assumed to be strictly positive. ${ }^{4}$ By rewriting equation (40) as follows

$$
\frac{\partial x^{*}}{\partial \lambda}=\vartheta f^{\prime}\left(Q^{*}\right) c^{\prime}\left(B x^{*}\right)\left\{\left(\beta+s^{\prime}(\lambda)\right)[\Lambda(1+\delta)+n]-[\tau+(n-1) s(\lambda)]\right\}
$$

where $\vartheta \equiv(n-1)\left(Q^{*} / n\right) / \tilde{\Delta}$ and $s(\lambda) \equiv \omega(\lambda)(\tilde{\beta}(\lambda)-\beta)$, we get that $\operatorname{sign}\left\{\partial x^{*} / \partial \lambda\right\}$ is given by (15). Let us now turn to the impact of $\lambda$ on output in equilibrium. Equation (39) can be rewritten as follows

$$
\frac{\partial q^{*}}{\partial \lambda}=\vartheta\left(\left(\beta+s^{\prime}(\lambda)\right) c^{\prime}\left(B x^{*}\right)^{2} B+f^{\prime}\left(Q^{*}\right)\left\{c^{\prime \prime}\left(B x^{*}\right)\left(Q^{*} / n\right) B[\tau+(n-1) s(\lambda)]+\Gamma^{\prime \prime}\left(x^{*}\right)\right\}\right) .
$$

By inserting the FOC (11) evaluated in the symmetric equilibrium into the above expression, after some manipulations we get that $\operatorname{sign}\left\{\partial q^{*} / \partial \lambda\right\}$ is given by (16). Finally, note that the FOC with respect to output is identical to the one associated to the static case. Therefore, we obtain again equation (7), which implies that if $\partial x^{*} / \partial \lambda \leq 0$, then $\partial q^{*} / \partial \lambda<0$. From (15), we obtain that $\partial x^{*} / \partial \lambda>0$ if and only if

$$
\beta>\underline{\beta}^{2 S} \equiv \frac{1-\left(\omega^{\prime}(\lambda) \tilde{\beta}(\lambda)+\omega(\lambda) \tilde{\beta}^{\prime}(\lambda)\right) P^{\prime}(c)^{-1} n+\omega(\lambda)(n-1) \tilde{\beta}(\lambda)}{(1+n+\Lambda \delta)+(n-1) \omega(\lambda)-P^{\prime}(c)^{-1} n \omega^{\prime}(\lambda)} .
$$

LEMMA 7 Under assumptions A.1.-A.4, in the two-stage model, there is a cut-off spillover value for spillovers $\left(\bar{\beta}^{2 S}<1\right)$ above which allowing some overlapping ownership is socially optimal $\left(\lambda_{\mathrm{TS}}^{o}>0\right)$ if

$$
\left(1+s^{\prime}(0)\right) n+(1-s(0))(n-1)\left(\left(1+s^{\prime}(0)\right)(1+\delta+n)-[1+(n-1) s(0)]-H(1)>0 .\right.
$$

\footnotetext{
${ }^{4}$ We show in Section A.2.2 that $\tilde{\Delta}\left(Q^{*}, x^{*}\right)>0$ is also a necessary condition for having a positive output at equilibrium in AJ.
} 
Proof. By differentiating $W(\lambda)$ we have

$$
W^{\prime}(\lambda)=\left[f\left(Q^{*}\right)-c\left(B x^{*}\right)\right] n \frac{\partial q^{*}}{\partial \lambda}-c^{\prime}\left(B x^{*}\right) B Q^{*} \frac{\partial x^{*}}{\partial \lambda}-n \Gamma^{\prime}\left(x^{*}\right) \frac{\partial x^{*}}{\partial \lambda} .
$$

Using the FOCs, $f\left(Q^{*}\right)-c\left(B x^{*}\right)=-f^{\prime}\left(Q^{*}\right) Q^{*} \Lambda / n$ and (14) in the above expression, and simplifying, we obtain:

$$
W^{\prime}(\lambda)=\left\{-\Lambda f^{\prime}\left(Q^{*}\right) \frac{\partial q^{*}}{\partial \lambda}-[(1-\lambda) \beta-s(\lambda)](n-1) c^{\prime}\left(B x^{*}\right) \frac{\partial x^{*}}{\partial \lambda}\right\} Q^{*}
$$

If we insert (41) and (42) into (44), after some manipulations we get

$$
\begin{aligned}
W^{\prime}(\lambda)= & \vartheta_{w} Q^{*}\left(-f^{\prime}\left(Q^{*}\right)\right)\left[\Lambda \left(c^{\prime}\left(B x^{*}\right)^{2}\left(\beta+s^{\prime}(\lambda)\right) B\right.\right. \\
& \left.+f^{\prime}\left(Q^{*}\right)\left\{c^{\prime \prime}\left(B x^{*}\right)\left(Q^{*} / n\right) B[\tau+(n-1) s(\lambda)]+\Gamma^{\prime \prime}\left(x^{*}\right)\right\}\right) \\
& +c^{\prime}\left(B x^{*}\right)^{2}[(1-\lambda) \beta-s(\lambda)](n-1)\left\{\left(\beta+s^{\prime}(\lambda)\right)[\Lambda(1+\delta)+n]\right. \\
& -[\tau+(n-1) s(\lambda)]\}]
\end{aligned}
$$

where $\vartheta_{w} \equiv(n-1)\left(Q^{*} / n\right) / \tilde{\Delta}$. Then $\left.W^{\prime}(0)\right|_{\beta=1}>0$ if and only if

$$
\begin{aligned}
0< & \left(c^{\prime}\left(n x^{*}\right)\right)^{2}\left(\left(1+\left.s^{\prime}(0)\right|_{\beta=1}\right) n+\left(1-\left.s(0)\right|_{\beta=1}\right)(n-1)\left\{\left(1+\left.s^{\prime}(0)\right|_{\beta=1}\right)(1+\delta+n)\right.\right. \\
& \left.\left.-\left[1+\left.(n-1) s(0)\right|_{\beta=1}\right]\right\}\right)+f^{\prime}\left(Q^{*}\right)\left\{c^{\prime \prime}\left(n x^{*}\right) Q^{*}\left[1+\left.(n-1) s(0)\right|_{\beta=1}\right]+\Gamma^{\prime \prime}\left(x^{*}\right)\right\} .
\end{aligned}
$$

From equation (14) we have that in equilibrium and for $\lambda=0$ and $\beta=1$ :

$$
\left.Q^{*}\right|_{\lambda=0, \beta=1}=-\frac{n \Gamma^{\prime}\left(x^{*}\right)}{c^{\prime}\left(n x^{*}\right)\left[1+\left.(n-1) s(0)\right|_{\beta=1}\right]} .
$$

Substituting $\left.Q^{*}\right|_{\lambda=0, \beta=1}$ into (46) and using the definitions for $\chi\left(B x^{*}\right)$ and $\xi\left(Q^{*}, x^{*}\right)$, we obtain the condition for the two-period model:

$$
\begin{aligned}
0< & \left(1+\left.s^{\prime}(0)\right|_{\beta=1}\right) n+\left(1-\left.s(0)\right|_{\beta=1}\right)(n-1)\left\{\left(1+\left.s^{\prime}(0)\right|_{\beta=1}\right)(1+\delta+n)\right. \\
& \left.-\left[1+\left.(n-1) s(0)\right|_{\beta=1}\right]\right\}-H(1),
\end{aligned}
$$

where

$$
s(0)=\frac{(2 n+\delta)[(n+\delta) /(2 n+\delta)-\beta]}{n(n+1+\delta)}
$$


Table 2: Model Specifications

\begin{tabular}{cc||c||c}
\hline & AJ & KMZ & CE \\
\hline Demand & $f(Q)=a-b Q$ & $f(Q)=a-b Q$ & $f(Q)=\sigma Q^{-\varepsilon}, 0<\varepsilon<1$ \\
& $\delta=0 ; a, b>0$ & $\delta=0 ; a, b>0$ & $\delta=-(1+\varepsilon) ; a=0, b=-\sigma<0$ \\
$c(\cdot)$ & $\bar{c}-x_{i}-\beta \sum_{j \neq i} x_{j}$ & $\bar{c}-\left[(2 / \gamma)\left(x_{i}+\beta \sum_{j \neq i} x_{j}\right)\right]^{1 / 2}$ & $\kappa\left(x_{i}+\beta \sum_{j \neq i} x_{j}\right)^{-\alpha} ; \alpha, \kappa>0$ \\
$\Gamma(x)$ & $(\gamma / 2) x^{2}$ & $x$ & $x$ \\
\hline
\end{tabular}

and

$$
s^{\prime}(0)=-\frac{\left[2 n^{2}+\delta(2 n+1)+\delta^{2}\right](n-1) \beta-\delta^{2}(n-1)-\delta\left(2 n^{2}-1\right)-n\left(n^{2}+1\right)}{(n+1+\delta)^{2} n} .
$$

Thus, $\left.s^{\prime}(0)\right|_{\beta=1}=[1+\delta-n(n-2)] /(n+1+\delta)^{2}$. Note that by setting $s=s^{\prime}=0$, we obtain the condition for the simultaneous case, that is, (31)

\section{A.2 The three model specifications}

In this section we characterize each of the model specifications considered in the paper: first in the simultaneous and then in the two-stage model. First we describe briefly the main assumptions of each model specification.

As shown in Amir (2000) the AJ and the KMZ model specifications are not equivalent for large spillover values (the critical value depends on the innovation function and on the number of firms). The difference between the two models lies on the innovation function and the autonomous R\&D expenditures. Under the KMZ specification, the effective R\&D investment for each firm is the sum of its own expenditure $x_{i}$ and a fixed fraction $(\beta)$ of the sum of the expenditures of the rest of firms, i.e., $X_{i}=x_{i}+\beta \sum_{j \neq i} x_{j}$. Instead, under the AJ specification, $X_{i}$ is the effective cost reduction for each firm, so $c(\cdot)$ is a linear function. Thus, in AJ decision variables are unit-cost reductions, whereas in KMZ decision variables are the autonomous R\&D expenditures. In particular, in KMZ the unit cost of firm $i$ is $\bar{c}-h\left(x_{i}+\beta \sum_{j \neq i} x_{j}\right)$, where for given $x_{i} \geq 0(i=1, \ldots, n)$ the effective cost reduction to firm $i, h(\cdot)$, is a twice differentiable and concave function with $h(0)=0, h(\cdot) \leq \bar{c}$, and $\left(\partial / \partial x_{i}\right) h(\cdot)>0$. As in Amir (2000), to allow for a direct comparison between AJ and KMZ, we consider a particular case of the KMZ model: $h=\left[(2 / \gamma)\left(x_{i}+\beta \sum_{j \neq i} x_{j}\right)\right]^{1 / 2}$ with $\gamma>0$. The CE model considers constant elasticity demand and costs with $\alpha, \kappa>0$ (see Table 2); $\alpha$ is the unit cost of production (or innovation function) elasticity with respect to the investment in $R \& D$ and there are no spillover effects. Note that the assumption $\varepsilon<1$ implies that $\delta>-2$, and consequently quantities are strategic substitutes. 
Finally, $\Gamma(x)$ is quadratic in AJ but linear in KMZ and CE.

\section{A.2.1 Simultaneous model}

We first discuss comparative statics on equilibrium values given in Table A1, and then derive Table A2, which provides the second-order and regularity conditions for the three model specifications (we also explore the feasible region for the constant elasticity model in Lemma A1). Second, we establish Lemma A2, which determines $\operatorname{sign}\left\{\partial q^{*} / \partial \lambda\right\}$ and $\operatorname{sign}\left\{\partial x^{*} / \partial \lambda\right\}$ for each model specification. Third, we derive the spillover threshold value $\bar{\beta}$ and $\beta^{\prime}(0)$ in the examples (Table A3). After that, we conduct a comparative statics analysis on $\bar{\beta}$. Finally, we examine welfare in AJ and KMZ, obtain the optimal degree of overlapping ownership in each case (Table A4) and state and prove Proposition A1.

Table A1: Equilibrium Values

\begin{tabular}{cc||c||c}
\hline & $A J$ & $K M Z$ & $C E$ \\
\hline$q^{*}$ & $\frac{\gamma(a-\bar{c})}{\gamma b(\Lambda+n)-B \tau}$ & $\frac{\gamma(a-\bar{c})}{\gamma b(\Lambda+n)-\tau}$ & $\frac{1}{\alpha \kappa \tau}\left[\sigma(\tau \alpha / n)^{\varepsilon} \kappa^{\varepsilon-1}(1-\varepsilon \Lambda / n)\right]^{(1+\alpha) /[\varepsilon-\alpha(1-\varepsilon)]}$ \\
$x^{*}$ & $\frac{\tau(a-\bar{c})}{\gamma b(\Lambda+n)-B \tau}$ & $\frac{\gamma \tau^{2}(a-\bar{c})^{2}}{2 B[\gamma b(\Lambda+n)-\tau]^{2}}$ & $\frac{1}{B}\left[\sigma(\tau \alpha / n)^{\varepsilon} \kappa^{\varepsilon-1}(1-\varepsilon \Lambda / n)\right]^{1 /[\varepsilon-\alpha(1-\varepsilon)]}$ \\
\hline
\end{tabular}

Table A2: Second-Order Conditions and Regularity Condition

\begin{tabular}{ll||l||l}
\hline \multicolumn{1}{c|}{$A J$} & \multicolumn{1}{c|}{$K M Z$} & \multicolumn{1}{c}{$C E$} \\
\hline S.O.C & $\gamma b>1 / 2$ & $\gamma b>\tau /(2 \tilde{\lambda})$ & $n>\frac{\Lambda(1+\varepsilon)}{2}$ and $\frac{\varepsilon(1+\alpha)}{\alpha}>\frac{n(n-\varepsilon \Lambda)}{\tilde{\lambda}(2 n+\Lambda \delta)}$ \\
Regularity Condition & $\gamma b>\tau B /(\Lambda+n)$ & $\gamma b>\tau /(\Lambda+n)$ & $\begin{array}{l} \\
\varepsilon-\alpha(1-\varepsilon)>0\end{array}$ \\
\hline
\end{tabular}

with $\tilde{\lambda} \equiv 1+\lambda(n-1) \beta^{2}$.

Table A3: Spillover Thresholds $\bar{\beta}$ and $\beta^{\prime}(0)$

\begin{tabular}{l|c|c} 
& $\bar{\beta}$ & $\beta^{\prime}(0)$ \\
\hline$A J$ & $\frac{(n-2)+\sqrt{(n-2)^{2}+4 b \gamma(n+2)(n-1)}}{2(n+2)(n-1)}$ & {$[-1+\sqrt{1+4 b \gamma(n-1)}] /[2(n-1)]$} \\
$K M Z$ & $\frac{(n-2)+b \gamma(n-1)+\sqrt{(n-2)^{2}+b \gamma(n-1)[b \gamma(n-1)+6 n+4]}}{2(n+2)(n-1)}$ & $\gamma b$ \\
$C E$ & $\begin{array}{l}\text { is the value above which: } \\
(n-\varepsilon) \alpha \beta\{B+(n-1)[\beta(n-\varepsilon)-1]\}-\varepsilon(\alpha+1) B>0\end{array}$ & $\varepsilon(\alpha+1) /[\alpha(n-\varepsilon)]$
\end{tabular}

Comparative statics on equilibrium values. In AJ and KMZ the R\&D expenditure $x^{*}$ and output $q^{*}$ per firm increase with the size of the market $(a)$ and decrease with the level of inefficiency of the technology employed, $\bar{c}$, the slope of inverse demand, $b$, and the parameter 
$\gamma$ (which is the parameter of the slope of the R\&D costs in AJ). In the CE model $x^{*}$ and $q^{*}$ increase with the size of the market, $\sigma$. In addition, the costlier the technology employed, $\kappa$, the lower is total output, $Q^{*}$. However, $x^{*}$ decreases (respectively, increases) with $\kappa$ if demand is elastic (inelastic). The last two results hold for any value of $\lambda$ and $\beta .^{5}$

Derivation of Table A2. In AJ and KMZ it is immediate that $\partial_{q_{i} q_{i}} \phi_{i}=-2 b<0$. Furthermore, in AJ: condition $\partial_{q_{i} q_{i}} \phi_{i}\left(\partial_{x_{i} x_{i}} \phi_{i}\right)-\left(\partial_{q_{i} x_{i}} \phi_{i}\right)^{2}>0$, given by (21), can be written as $2 b \gamma-1>0$, since $c^{\prime \prime}(\cdot)=0$ and $\Gamma^{\prime \prime}(x)=\gamma$, so $\partial_{x_{i} x_{i}} \phi_{i}=-\gamma$ and $\partial_{q_{i} x_{i}} \phi_{i}=-c^{\prime}(\cdot)=1$. In KMZ, (21) can be written as

$$
\left[\frac{1}{\gamma^{2}}\left(\frac{2}{\gamma}\left(B x^{*}\right)\right)^{-1}\right]-2 b\left[\frac{1}{\gamma^{2}}\left(\frac{2}{\gamma}\left(B x^{*}\right)\right)^{-3 / 2}\right] q^{*} \tilde{\lambda}<0
$$

From FOC (3) we have that in equilibrium

$$
q^{*}=\frac{\Gamma^{\prime}\left(x^{*}\right)}{-c^{\prime}\left(B x^{*}\right) \tau}=\frac{1}{(1 / \gamma)\left[\left(2\left(B x^{*}\right) / \gamma\right]^{-1 / 2} \tau\right.} .
$$

Inserting the above equation into condition (47), after some manipulations, it reduces to 1 $2 b \gamma \tilde{\lambda} / \tau<0$. (Note that if $\gamma b>\tau / 2$ holds, then the condition $\gamma b>\tau /(2 \tilde{\lambda})$ is satisfied.) In AJ and from $(20)$, it is immediate that $\Delta=\gamma b(\Lambda+n)-\tau B$ since $c^{\prime \prime}(\cdot)=\delta=0, f^{\prime}(Q)=-b$ and $\Gamma^{\prime}(x)=\gamma x$. In KMZ we have:

$$
\begin{aligned}
\Delta & =-\left[\frac{1}{\gamma^{2}}\left(\frac{2}{\gamma} B x^{*}\right)^{-3 / 2} B \tau \frac{1}{(1 / \gamma)\left(2 B x^{*} / \gamma\right)^{-1 / 2} \tau}\right][-b(\Lambda+n)]-\frac{1}{\gamma^{2}}\left(\frac{2}{\gamma} B x^{*}\right)^{-1} \tau B \\
& =\frac{1}{\gamma}\left(\frac{2}{\gamma} B x^{*}\right)^{-1}\left[B b(\Lambda+n)-\frac{\tau B}{\gamma}\right] .
\end{aligned}
$$

Therefore, in KMZ $\Delta>0$ if $\gamma b>\tau /(\Lambda+n)$. Regarding the constant elasticity model we have:

LEMMA A1 (Constant elasticity model) At the equilibrium, for a given $n \geq 2$ and $\lambda \geq 0$, second order conditions together with the condition of non-negative profits require that

(i) $\max \{\varepsilon \Lambda, \Lambda(1+\varepsilon) / 2\}<n \leq \varepsilon \Lambda(B+\alpha \tau) /(\alpha \tau)$,

(ii) $\varepsilon(1+\alpha) / \alpha>n(n-\varepsilon \Lambda) /[\tilde{\lambda}(2 n+\Lambda \delta)]$, with $\tilde{\lambda} \equiv 1+\lambda(n-1) \beta^{2}$.

Furthermore, the equilibrium is regular if and only if $(1+\alpha) / \alpha>1 / \varepsilon$.

Proof. From the FOC (2) we need that

$$
n>\varepsilon \Lambda,
$$

\footnotetext{
${ }^{5}$ The same result is obtained in Dasgupta and Stiglitz (1980) for $\lambda=\beta=0$ and free entry.
} 
otherwise the system $(2 ; 3)$ will not have a solution. Since $\delta=-(1+\varepsilon), \Delta_{q}<0$ if condition (49) holds (see Table 4 ). This condition also guarantees that $Q^{*}$ and $x^{*}$ are both positive. Notice that $\partial_{q_{i} q_{i}} \phi_{i}<0$ if $\left(f^{\prime}\left(Q^{*}\right) / n\right)(2 n+\Lambda \delta)<0$, then $\partial_{q_{i} q_{i}} \phi_{i}<0$ if

$$
n>\Lambda(1+\varepsilon) / 2
$$

Since $\Lambda \in[1, n]$, we have that the latter condition is always satisfied for $\varepsilon<1$. By construction $\partial_{x_{i} x_{i}} \phi_{i}<0$. Furthermore, second order condition $\partial_{q_{i} q_{i}} \phi_{i}\left(\partial_{x_{i} x_{i}} \phi_{i}\right)-\left(\partial_{q_{i} x_{i}} \phi_{i}\right)^{2}>0$, which is given by (21), reduces to

$$
-\frac{\varepsilon \sigma}{n} Q^{*-(\varepsilon+1)}(2 n+\Lambda \delta)\left[\alpha(\alpha+1) \kappa\left(B x^{*}\right)^{-(\alpha+2)}\left(Q^{*} / n\right) \tilde{\lambda}\right]+(\alpha \kappa)^{2}\left(B x^{*}\right)^{-2(\alpha+1)}<0 .
$$

From the FOC (2) we have that at the symmetric equilibrium

$$
Q^{*}=[\sigma(n-\varepsilon \Lambda) /(n \kappa)]^{1 / \varepsilon}\left(B x^{*}\right)^{\alpha / \varepsilon} .
$$

By substituting (52) into (51), after some manipulations, we obtain

$$
\left(B x^{*}\right)^{-2(\alpha+1)} \alpha \kappa^{2}\{-[\varepsilon /(n-\varepsilon \Lambda)](2 n+\Lambda \delta)(\alpha+1) \tilde{\lambda} / n+\alpha\}<0 .
$$

The above condition is satisfied if $\varepsilon(\alpha+1) / \alpha>n(n-\varepsilon \Lambda) /[(2 n+\Lambda \delta) \tilde{\lambda}]$, which proves statement (ii) of the Proposition.

From (20) we have that $\Delta>0$ if

$$
\begin{aligned}
0< & -\alpha(\alpha+1) \kappa\left(B x^{*}\right)^{-(\alpha+2)}\left(Q^{*} / n\right) \tau B\left[\varepsilon(1+\varepsilon) \sigma Q^{*-(\varepsilon+2)} \Lambda Q^{*}-\varepsilon \sigma Q^{*-(\varepsilon+1)}(\Lambda+n)\right] \\
& -(\alpha \kappa)^{2}\left(B x^{*}\right)^{-2(\alpha+1)} \tau B, \text { or } \\
0< & Q^{*-(\varepsilon+1)}\left[-\alpha(\alpha+1) \kappa\left(B x^{*}\right)^{-(\alpha+2)}\left(Q^{*} / n\right) \tau B\right][\varepsilon(1+\varepsilon) \sigma \Lambda-\varepsilon \sigma(\Lambda+n)] \\
& -(\alpha \kappa)^{2}\left(B x^{*}\right)^{-2(\alpha+1)} \tau B .
\end{aligned}
$$

Substituting (52) into the above expression, we obtain

$$
\begin{aligned}
0< & -\left[\frac{\sigma(n-\varepsilon \Lambda)}{n \kappa}\right]^{-(\varepsilon+1) / \varepsilon}\left(B x^{*}\right)^{-(\varepsilon+1) \alpha / \varepsilon} \alpha(\alpha+1) \kappa\left(B x^{*}\right)^{-(\alpha+2)}\left[\frac{\sigma(n-\varepsilon \Lambda)}{n \kappa}\right]^{1 / \varepsilon}\left(B x^{*}\right)^{\alpha / \varepsilon} \\
& \frac{\tau B}{n}[\varepsilon(1+\varepsilon) \sigma \Lambda-\varepsilon \sigma(\Lambda+n)]-(\alpha \kappa)^{2}\left(B x^{*}\right)^{-2(\alpha+1)} \tau B,
\end{aligned}
$$


rearranging terms yields

$0<\left(B x^{*}\right)^{-2(\alpha+1)}\left[\frac{n \kappa}{\sigma(n-\varepsilon \Lambda)}\left(-\alpha(\alpha+1) \frac{\kappa \tau B}{n}\right)\left(-\varepsilon \sigma n+\varepsilon^{2} \sigma \Lambda\right)-(\alpha \kappa)^{2} \tau B\right]$, or equivalently,

$$
0<\left(B x^{*}\right)^{-2(\alpha+1)} \alpha \kappa^{2} \tau B[\varepsilon(\alpha+1)-\alpha]
$$

Therefore, $\Delta>0$ holds if $(1+\alpha) / \alpha>1 / \varepsilon$, or, equivalently, if $\varepsilon-\alpha(1-\varepsilon)>0$.

We turn now to deriving the condition under which profits in equilibrium are nonnegative. At the symmetric equilibrium, each firm's profit is given by $\pi\left(Q^{*} / n, x^{*}\right)=\left[f\left(Q^{*}\right)-c\left(B x^{*}\right)\right]\left(Q^{*} / n\right)-$ $x^{*}$. Then, $\pi\left(Q^{*} / n, x^{*}\right) \geq 0$ if $\bar{\pi} \equiv\left[f\left(Q^{*}\right)-c\left(B x^{*}\right)\right] Q^{*} /\left(x^{*} n\right) \geq 1$. Write

$$
\vartheta_{C E} \equiv \sigma\left(\frac{\tau \alpha}{n}\right)^{\varepsilon} \kappa^{\varepsilon-1}\left(\frac{n-\varepsilon \Lambda}{n}\right)
$$

Then $Q^{*}=[n /(\alpha \kappa \tau)] \vartheta_{C E}{ }^{(1+\alpha) /[\varepsilon-\alpha(1-\varepsilon)]}, x^{*}=(1 / B) \vartheta_{C E}{ }^{1 /[\varepsilon-\alpha(1-\varepsilon)]}$, and condition $\bar{\pi} \geq 1$ can be expressed as

$$
\left[\sigma\left(\frac{n}{\alpha \kappa \tau}\right)^{-\varepsilon} \vartheta_{C E}{ }^{-\varepsilon(1+\alpha) /[\varepsilon-\alpha(1-\varepsilon)]}-\kappa \vartheta_{C E}{ }^{-\alpha /[\varepsilon-\alpha(1-\varepsilon)]}\right] \frac{1}{\alpha \kappa \tau} \vartheta_{C E}{ }^{\alpha /[\varepsilon-\alpha(1-\varepsilon)]} B \geq 1
$$

Rearranging terms, and replacing $\vartheta_{C E}$ into the above expression, we get $[\varepsilon \Lambda /(n-\varepsilon \Lambda)][B /(\alpha \tau)] \geq$ 1. It follows that $\bar{\pi} \geq 1$ if

$$
\left(\frac{\varepsilon \Lambda}{\alpha \tau}\right)(B+\alpha \tau) \geq n
$$

Combining conditions (49), (50) and (53) yields statement (i) 


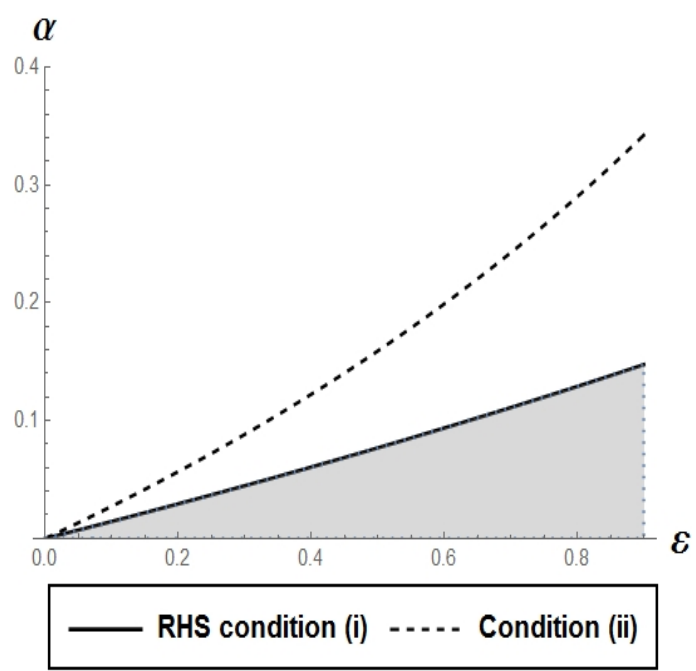

Fig. A1. Feasible region for the CE model with $n=7$.

Feasible region for the constant elasticity model with $\lambda=0$. From Lemma A1 we have that $\Delta>0$ if $(1+\alpha) / \alpha>1 / \varepsilon$. When $\lambda=0$, the LHS of condition (i) is satisfied for any $n \geq 2$ since $\varepsilon<1$, moreover the RHS of condition (i) can be rewritten as follows $n \leq \rho_{C E}(\beta)=\varepsilon(1+\alpha-\beta) /(\alpha-\varepsilon \beta)$. Since $\rho_{C E}^{\prime}>0$ (as we are also imposing that $\Delta>0$ ), condition $n \leq \rho_{C E}(\beta)$ will hold for all $\beta$ if $n \leq \varepsilon(1+\alpha) / \alpha$. Last, condition (ii) with $\lambda=0$ writes as $\varepsilon(1+\alpha) / \alpha>n(n-\varepsilon) /[2 n-(1+\varepsilon)]$. Therefore, at $\lambda=0$ we only have to consider the RHS of condition (i) and condition (ii). These two conditions are depicted in Fig. A1 for $n=7$; the grey area are combinations $(\alpha, \varepsilon)$ for which the two conditions are satisfied (these combinations of parameters also satisfy the two conditions for $n \leq 7$ ).

Determination of $\operatorname{sign}\left\{\partial q^{*} / \partial \lambda\right\}$ and $\operatorname{sign}\left\{\partial x^{*} / \partial \lambda\right\}$ in AJ, KMZ and CE. Note that $\partial q^{*} / \partial \lambda$ can be written in the following manner

$$
\frac{\partial q^{*}}{\partial \lambda}=\frac{(n-1)\left(Q^{*} / n\right)}{\Delta}\left\{\left(c^{\prime}\left(B x^{*}\right)\right)^{2} \beta B+f^{\prime}\left(Q^{*}\right)\left[c^{\prime \prime}\left(B x^{*}\right)\left(Q^{*} / n\right) B \tau+\Gamma^{\prime \prime}\left(x^{*}\right)\right]\right\},
$$

then after some calculations, it is simple to verify that in the simultaneous model:

LEMMA A2 We have (i) In AJ: $\operatorname{sign}\left\{\frac{\partial q^{*}}{\partial \lambda}\right\}=\operatorname{sign}\{\beta(1+\beta(n-1))-b \gamma\}$ and $\operatorname{sign}\left\{\frac{\partial x^{*}}{\partial \lambda}\right\}=$ $\operatorname{sign}\{\beta(n+1)-1\}$; (ii) In KMZ: $\operatorname{sign}\left\{\frac{\partial q^{*}}{\partial \lambda}\right\}=\operatorname{sign}\{\beta-\gamma b\}$ and $\operatorname{sign}\left\{\frac{\partial x^{*}}{\partial \lambda}\right\}=\operatorname{sign}\{\beta(n+$ 1) -1$\}$; (iii) In the CE model: $\operatorname{sign}\left\{\frac{\partial q^{*}}{\partial \lambda}\right\}=\operatorname{sign}\{\beta[\alpha(n-\varepsilon \Lambda)-\lambda(n-1) \varepsilon(\alpha+1)]-\varepsilon(\alpha+1)\}$ 
and $\operatorname{sign}\left\{\frac{\partial x^{*}}{\partial \lambda}\right\}=\operatorname{sign}\{\beta[(n-\varepsilon)-\lambda(n-1)(1+\varepsilon)]-1\}$.

Derivation of $\overline{\boldsymbol{\beta}}$ (Table A3). Note that $\partial x^{*} / \partial \lambda$ can be written as

$$
\frac{\partial x^{*}}{\partial \lambda}=\frac{(n-1)\left(Q^{*} / n\right) f^{\prime}\left(Q^{*}\right) c^{\prime}\left(B x^{*}\right)}{\Delta}[\beta(\Lambda(1+\delta)+n)-\tau]
$$

If we insert equations (54) and (55) into equation (9), after some manipulations we obtain $W^{\prime}(\lambda)=\left[(n-1)\left(Q^{*}\right)^{2} /(n \Delta)\right]\left(-f^{\prime}\left(Q^{*}\right)\right) \digamma$, where

$$
\begin{aligned}
\digamma \equiv & \Lambda\left\{\left(c^{\prime}\left(B x^{*}\right)\right)^{2} \beta B+f^{\prime}\left(Q^{*}\right)\left[c^{\prime \prime}\left(B x^{*}\right)\left(Q^{*} / n\right) B \tau+\Gamma^{\prime \prime}\left(x^{*}\right)\right]\right\} \\
& +\left(c^{\prime}\left(B x^{*}\right)\right)^{2}(1-\lambda) \beta(n-1)\{\beta[\Lambda(1+\delta)+n]-\tau\} .
\end{aligned}
$$

By noting that in AJ: $f^{\prime}=-b, \delta=0, c^{\prime}=-1, c^{\prime \prime}=0$ and $\Gamma^{\prime \prime}=\gamma$, it then follows that

$$
\begin{aligned}
\digamma^{A J} & =\left.\digamma\right|_{\lambda=0}=\beta B-b \gamma+\beta(n-1)[\beta(1+n)-1] \\
& =(n-1)(n+2) \beta^{2}-(n-2) \beta-b \gamma
\end{aligned}
$$

By solving $\digamma^{A J}=0$ for $\beta$ we obtain the expression for $\bar{\beta}^{A J}$. Notice that $\bar{\beta}^{A J}<1$ if

$$
(n-2)+\sqrt{(n-2)^{2}+4 b \gamma(n+2)(n-1)}<2(n+2)(n-1),
$$

or

$$
(n-2)^{2}+4 b \gamma(n+2)(n-1)<[2(n+2)(n-1)-(n-2)]^{2},
$$

which can be rewritten as $4 b \gamma(n+2)(n-1)<4 n^{2}(n+2)(n-1)$. Thus, $\bar{\beta}^{A J}<1$ if $b \gamma<n^{2}$. In KMZ we have $c=\bar{c}-\sqrt{(2 / \gamma)\left(x_{i}+\beta \sum_{j \neq i} x_{j}\right)}, f^{\prime}=-b, \delta=0$ and $\Gamma^{\prime \prime}=0$, then

$$
\begin{aligned}
\digamma^{K M Z} & =\left.\digamma\right|_{\lambda=0}=\frac{\beta}{2 \gamma x^{*}}+\frac{-b q^{*} B}{\gamma^{2}\left(2 B x^{*} / \gamma\right)^{3 / 2}}+\frac{\beta(n-1)[\beta(1+n)-1]}{2 \gamma B x^{*}} \\
& =\frac{1}{B}\left(\frac{-b q^{*} B^{1 / 2}}{\gamma^{2}\left(2 x^{*} / \gamma\right)^{3 / 2}}+\frac{\beta}{2 \gamma x^{*}}\{B+(n-1)[\beta(1+n)-1]\}\right) .
\end{aligned}
$$

By replacing $q^{*}$ and $x^{*}$ into the above expression, after some calculations we get

$$
\digamma^{K M Z}=\frac{[b \gamma(1+n)-1]^{2}}{\gamma(a-\bar{c})^{2}}\left(-b B+\frac{\beta}{\gamma}\{B+(n-1)[\beta(1+n)-1]\}\right) .
$$


It is then immediate that: $\digamma^{K M Z}>0 \Leftrightarrow \beta>\bar{\beta}^{K M Z}$. Notice that $\bar{\beta}^{K M Z}<1$ if

$$
\left\{(n-2)^{2}+b \gamma(n-1)[b \gamma(n-1)+2(3 n+2)]\right\}^{1 / 2}<2(n+2)(n-1)-n+2-b \gamma(n-1),
$$

which can be rewritten as $4 n(n+2)(n-1)(-n+b \gamma)<0$. In the constant elasticity model $f=\sigma Q^{-\varepsilon}, c=\kappa\left(x_{i}+\beta \sum_{j \neq i} x_{j}\right)^{-\alpha}$ and $\Gamma(x)=x$, then

$$
\begin{aligned}
\digamma^{C E}= & \left.\digamma\right|_{\lambda=0}=(\alpha \kappa)^{2}\left(B x^{*}\right)^{-2(\alpha+1)} \beta B-\varepsilon \sigma\left(Q^{*}\right)^{-\varepsilon-1} \alpha(\alpha+1) \kappa\left(B x^{*}\right)^{-(\alpha+2)} q^{*} B \\
& +(\alpha \kappa)^{2}\left(B x^{*}\right)^{-2(\alpha+1)} \beta(n-1)[\beta(-\varepsilon+n)-1] .
\end{aligned}
$$

By replacing $q^{*}$ and $x^{*}$ into the above expression, we obtain

$$
\begin{aligned}
\digamma^{C E}= & \alpha^{2} \kappa^{2} z^{-2(1+\alpha)} \beta B-\varepsilon \sigma[n /(\alpha \kappa)]^{-(1+\varepsilon)} z^{-(1+\alpha)(1+\varepsilon)}(\alpha+1) z^{-(\alpha+2)} z^{\alpha+1} B \\
& +\alpha^{2} \kappa^{2} z^{-2(1+\alpha)} \beta(n-1)[\beta(-\varepsilon+n)-1],
\end{aligned}
$$

where

$$
z \equiv\left[\sigma\left(\frac{\tau \alpha}{n}\right)^{\varepsilon} \kappa^{\varepsilon-1}(1-\varepsilon / n)\right]^{1 /[\varepsilon-\alpha(1-\varepsilon)]} .
$$

By noting that $z^{-(\alpha+1)(1+\varepsilon)-(\alpha+2)+(\alpha+1)}=z^{-\varepsilon+\alpha(1-\varepsilon)} z^{-2(1+\alpha)}$ we can re-write equation (56) as follows

$$
\digamma^{C E}=z^{-2(1+\alpha)} \alpha \kappa^{2}\{\alpha \beta B+\alpha \beta(n-1)[\beta(-\varepsilon+n)-1]-\varepsilon(\alpha+1) B /(n-\varepsilon)\} .
$$

Hence $\digamma^{C E}>0$ if and only if

$$
(n-\varepsilon) \alpha \beta\{B+(n-1)[\beta(n-\varepsilon)-1]\}-\varepsilon(\alpha+1) B>0 .
$$




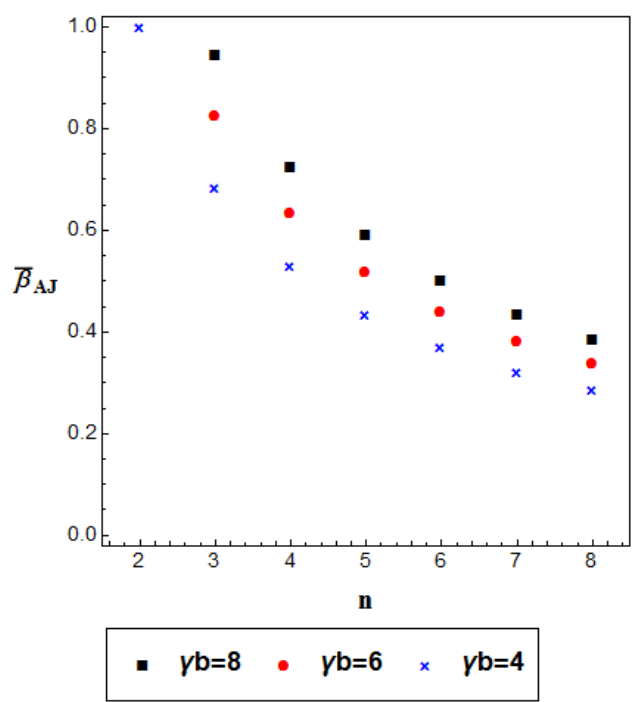

Fig. A2a. AJ model.

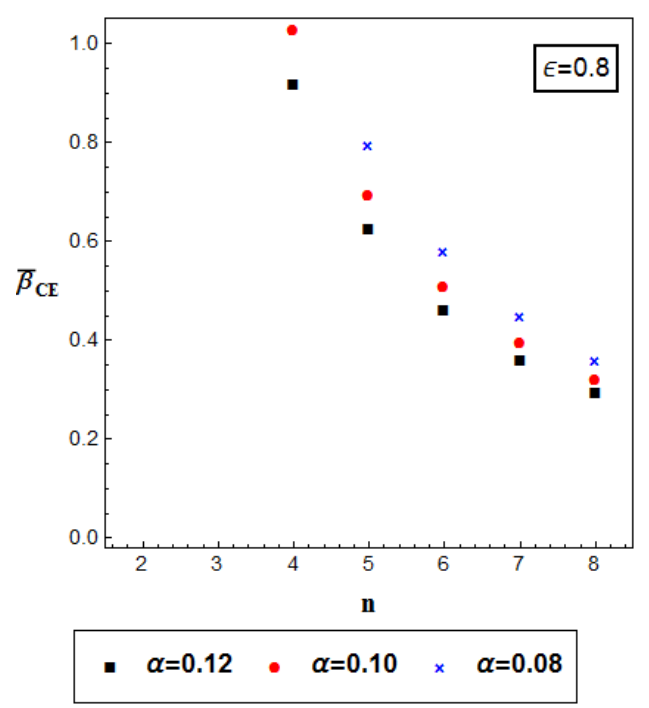

Figure A3a. CE model.

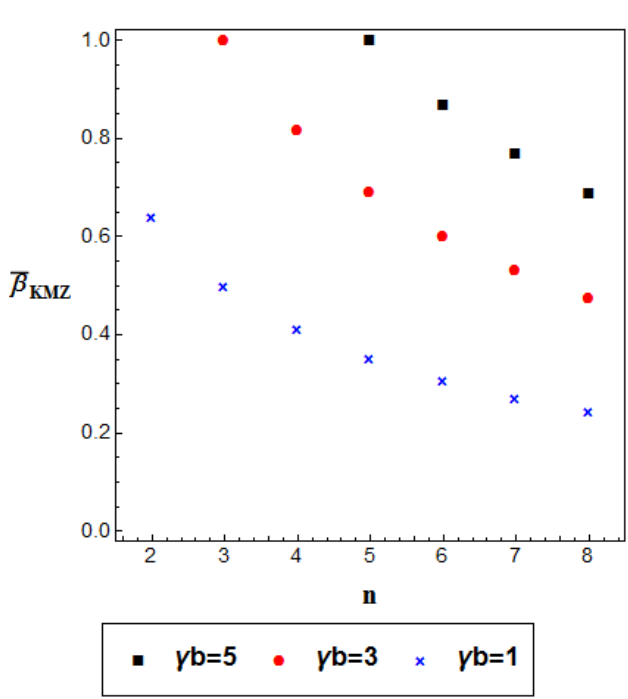

Fig. A2b. KMZ model.

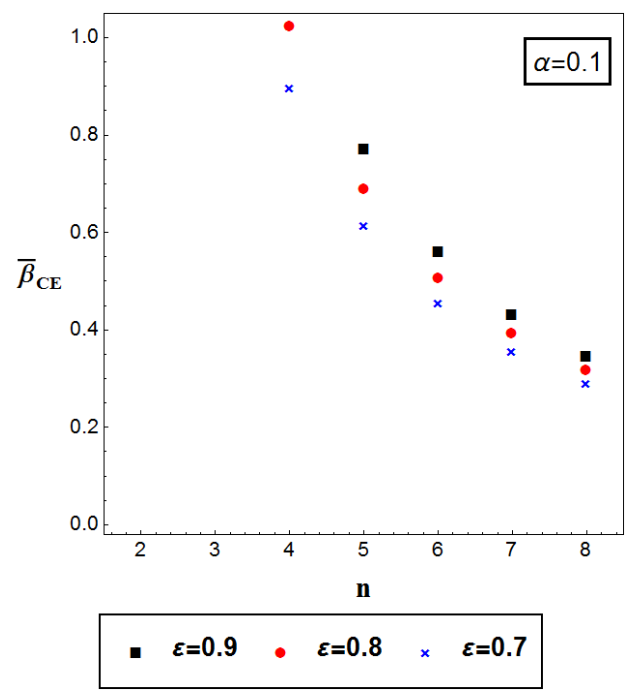

Figure A3b. CE model.

Comparative statics on $\bar{\beta}$. Fig. A2a (respectively Fig. A2b) shows the value for $\bar{\beta}$ under the AJ (KMZ) model specification as a function of the number of firms and for different values of $\gamma b$. As the figure makes clear, $\bar{\beta}^{A J}$ and $\bar{\beta}^{K M Z}$ decrease with $n$ : when there are more firms in the market, there is more need for overlapping ownership in order to internalize the additional externalities. We also have that $\bar{\beta}^{A J}$ and $\bar{\beta}^{K M Z}$ decrease with $\gamma b$, although $\bar{\beta}$ is lower than 1 for lower values of $\gamma b$ in the KMZ model than in the AJ model.

Fig. A3a and Fig. A3b depict $\bar{\beta}^{C E}$ as a function of $n$ and for different values for $\alpha$ and $\varepsilon$. A glance at these figures shows that $\bar{\beta}^{C E}$ decreases again with $n$ (for given $\varepsilon$ and $\alpha$ ). In 
Table A4: Optimal Degree of Cross-ownership in AJ and KMZ $\lambda_{\mathrm{TS}}^{o}$
AJ $\quad \min \left\{\max \left\{0, \frac{[(n+2)(n-1) \beta-(n-2)] \beta-b \gamma}{(n-1)[2(\beta-1) \beta+b \gamma]}\right\}, 1\right\}$
$\mathrm{KMZ} \min \left\{\max \left\{0, \frac{[(n+2)(n-1) \beta-b \gamma(n-1)-(n-2)] \beta-b \gamma}{(n-1)\{[2 \beta+b \gamma(n-1)-2] \beta+b \gamma\}}\right\}, 1\right\}$

addition, Fig. A3a tells us that for given $n$ and $\varepsilon, \bar{\beta}^{C E}$ decreases with the elasticity of the innovation function, $\alpha$, whereas Fig. A3b shows that for given $n$ and $\alpha, \bar{\beta}^{C E}$ increases with $\varepsilon$, so it decreases with the elasticity of demand. We also have that for the (feasible) combination of parameters $(\alpha, \varepsilon)$ considered here, $\bar{\beta}^{C E} \geq 1$ when there are two or three firms in the market.

Optimal degree of overlapping ownership (TS and CS standard)

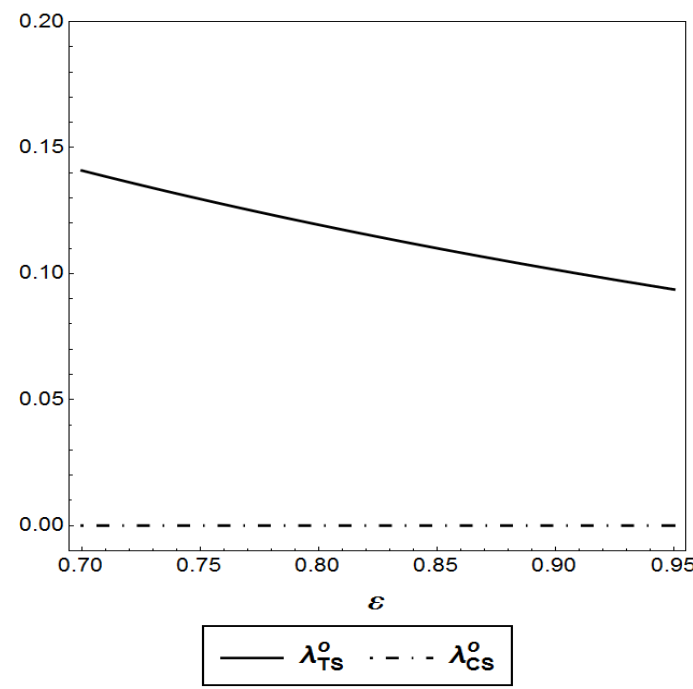

Fig. A4a. CE model.

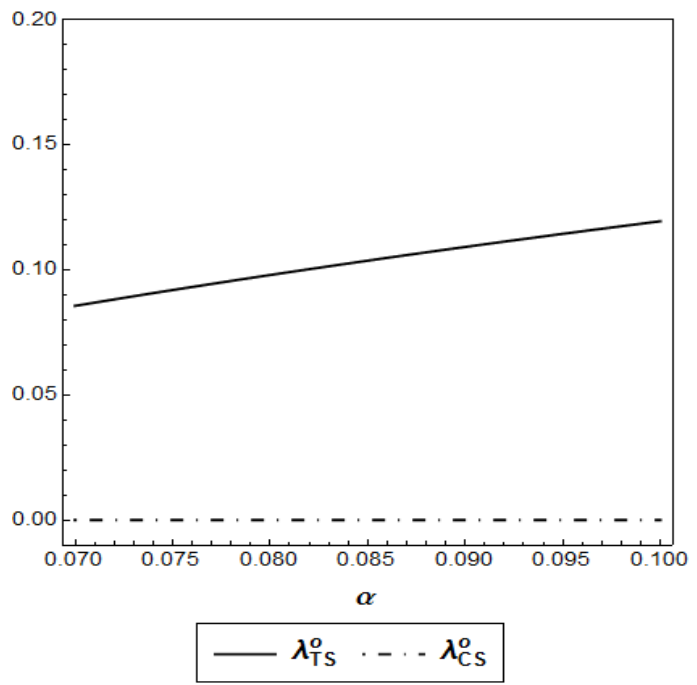

Fig. A4b. CE model. $(\varepsilon=0.8, \sigma=\kappa=1, n=8, \beta=0.8)$

$(\alpha=0.1, \sigma=\kappa=1, n=8, \beta=0.8)$

Fig. A4a and A4b show that the greater is the elasticity of demand, $\varepsilon^{-1}$, or the elasticity of the innovation function, $\alpha$, the greater should be the degree of overlapping ownership if the social planner seeks to maximize total surplus; however, if the objective is to maximize consumer surplus, then for the same parameter range, $\lambda_{\mathrm{CS}}^{o}=0$.

Welfare in AJ and KMZ. Here, we show that welfare is a single-peaked function in AJ and KMZ; we also derive $\lambda_{\text {TS }}^{o}$ under these two model specifications (Table A4). 
Case AJ: By inserting equilibrium values into the welfare function we get

$$
W=\frac{1}{2} n \gamma(a-\bar{c})^{2} \frac{(2 \Lambda+n) \gamma b-\tau^{2}}{[(\Lambda+n) \gamma b-B \tau]^{2}}
$$

If we differentiate $W$ with respect to $\lambda$ we obtain:

$$
\frac{d W}{d \lambda}=-\frac{(n-1)(a-\bar{c}) \gamma b\{\Lambda \gamma b+\beta[2 \lambda(B-n)+n-2-\beta(n+2)(n-1)]\}}{[(\Lambda+n) b \gamma-B \tau]^{2}} Q .
$$

Note that solving $d W / d \lambda=0$ for $\lambda$ yields a unique stationary point, given by $\hat{\lambda}_{A J}$. By taking the second order derivative with respect to $\lambda$, evaluating it at $\lambda=\hat{\lambda}_{A J}$, and simplifying, we obtain

$$
\left.\frac{d^{2} W}{d \lambda^{2}}\right|_{\lambda=\hat{\lambda}_{A J}}=-\frac{(n-1)^{2}(a-\bar{c}) \gamma b[2(\beta-1) \beta+\gamma b]^{3}}{\left[-(n+2)(n-1)^{2} \beta^{4}-6(n-1) \beta^{3}+Z_{1}+2 Z_{2}-Z_{3}\right]^{2}} Q,
$$

where $Z_{1} \equiv\left[\left(n^{2}+4 n-1\right) \gamma b+3(n-2)\right] \beta^{2}, Z_{2} \equiv 2[\gamma b(1-2 n)+1] \beta$ and $Z_{3} \equiv \gamma b(1-\gamma b n)$. The second order condition requires that $\gamma b>1 / 2$ (see Table A2), then $2(\beta-1) \beta+\gamma b>0$ for any $\beta \in[0,1]$, and as a result: $d^{2} W /\left.d \lambda^{2}\right|_{\lambda=\hat{\lambda}_{A J}}<0$. Since $\hat{\lambda}_{A J}$ is the unique stationary point of $W$, it follows that $\hat{\lambda}_{A J}$ is a global maximum. This is the desired $\lambda_{\mathrm{TS}}^{o}$.

Case KMZ: By inserting equilibrium values into the welfare function we get

$$
W=\frac{1}{2} n \gamma(a-\bar{c})^{2} \frac{(2 \Lambda+n) B \gamma b-\tau^{2}}{[(\Lambda+n) \gamma b-\tau]^{2} B}
$$

By differentiating $W$ with respect to $\lambda$ we obtain:

$$
\frac{d W}{d \lambda}=-\frac{(n-1)(a-\bar{c}) \gamma b\{\Lambda B \gamma b+\beta[2 \lambda(B-n)+n-2-\beta(n+2)(n-1)]\}}{B[(\Lambda+n) b \gamma-\tau]^{2}} Q,
$$

and by solving $d W / d \lambda=0$ for $\lambda$ we get a unique stationary point, given by $\hat{\lambda}_{K M Z}$. The second order derivative with respect to $\lambda$ evaluated at $\lambda=\hat{\lambda}_{K M Z}$ yields

$$
\left.\frac{d^{2} W}{d \lambda^{2}}\right|_{\lambda=\hat{\lambda}_{K M Z}}=\frac{\gamma b(n-1)^{2}(a-\bar{c})}{B[(\Lambda+n) \gamma b-\tau]^{3}} Z_{K M Z} Q
$$

where $Z_{K M Z} \equiv-[\beta n+(1-\beta)] n(\gamma b)^{2}+\left[4 \beta(1-\beta) n+(1-\beta)^{2}-\beta^{2} n^{2}\right] \gamma b+\beta B[\beta(n+2)-2]$. The regulatory condition requires that $\gamma b>\tau /(\Lambda+n)$ (see Table A2), thus $d^{2} W /\left.d \lambda^{2}\right|_{\lambda=\hat{\lambda}_{K M Z}}<$ 0 whenever $Z_{K M Z}<0$. Since $\hat{\lambda}_{K M Z}$ is the unique stationary point of $W$, it follows that $\hat{\lambda}_{K M Z}$ is a global maximum whenever $Z_{K M Z}<0$. This is the desired $\lambda_{\mathrm{TS}}^{o}$. It is straightforward to show that the regularity condition is stricter than the second order condition under the KMZ model specification for $n>2$ (see Table A2). In addition, the regularity condition becomes stricter 
as $\lambda$ and $n$ increase. For $\lambda=1$, the maximum value of the right-hand side of the regularity condition is $\sqrt{n}(n-1) /[4(n-\sqrt{n})]$, which for example equals 0.60 for $n=2$ and 0.68 for $n=3$. Numerical simulations show that assuming $\gamma b>0.62$ guarantees that $Z_{K M Z}<0$ holds for any $n$; thus, $Z_{K M Z}<0$ is a mild condition: it is slightly stricter than the regularity condition in duopoly but softer for oligopoly of three or more firms.

PROPOSITION A1 A Research Joint Venture with no overlapping ownership $(\lambda=0$ and $\beta=1$ ) is socially optimal in $A J$ when $\gamma b \geq n^{2}$, in $K M Z$ when $\gamma b \geq n$, and in $C E$ (provided that $W(\lambda)$ is single peaked) when $\alpha \geq \varepsilon n /\left[(n-1) \varepsilon^{2}+\left(-1+n-2 n^{2}\right) \varepsilon+n\left(n^{2}+1-n\right)\right]$.

Proof. When $W(\lambda)$ is single peaked, $\bar{\beta}$ is the minimum threshold above which allowing some positive $\lambda$ is welfare enhancing (Proposition 4). Consequently, $\lambda_{\mathrm{TS}}^{o}=0$ for any $\beta \in[0,1]$ if $\bar{\beta} \geq 1$. From Table A3 we have that $\bar{\beta}_{A J} \geq 1$ if $\gamma b \geq n^{2}$ and $\bar{\beta}_{K M Z} \geq 1$ if $\gamma b \geq n$; in both cases $W(\lambda)$ is single peaked (see above). Also, from Table A3 we obtain $\bar{\beta}_{C E}$, and solving $\bar{\beta}_{C E}=1$ for $\alpha$, yields the threshold value in terms of $n$ and $\varepsilon$ : $\bar{\beta}_{C E} \geq 1$ if $\alpha \geq$ $\varepsilon n /\left[(n-1) \varepsilon^{2}+\left(-1+n-2 n^{2}\right) \varepsilon+n\left(n^{2}+1-n\right)\right]$. Next we show that for $\lambda=0, W^{\prime}(\beta)>0$ under AJ, KMZ and CE model specifications, and therefore it is socially optimal to set $\beta=1$ in the three cases. We can write

$$
\begin{aligned}
\frac{\partial W}{\partial \beta}= & \left(f\left(Q^{*}\right) n-n c\left(B x^{*}\right)\right) \frac{\partial q^{*}}{\partial \beta}-n c^{\prime}\left(B x^{*}\right)(n-1) x^{*} q^{*}-n c^{\prime}\left(B x^{*}\right) B \frac{\partial x^{*}}{\partial \beta} q^{*} \\
& -n \Gamma^{\prime}\left(x^{*}\right) \frac{\partial x^{*}}{\partial \beta} \\
= & {\left[-\Lambda f^{\prime}\left(Q^{*}\right) \frac{\partial q^{*}}{\partial \beta}-(1-\lambda) \beta(n-1) c^{\prime}\left(B x^{*}\right) \frac{\partial x^{*}}{\partial \beta}-c^{\prime}\left(B x^{*}\right)(n-1) x^{*}\right] Q^{*} . }
\end{aligned}
$$

In AJ and for $\lambda=0, \partial q^{*} / \partial \beta>0$ and $\partial x^{*} / \partial \beta>0$ (see Table A1), thus from (57) it is clear that $\partial W / \partial \beta>0$. In KMZ and for $\lambda=0, \partial q^{*} / \partial \beta=0$ and $\partial x^{*} / \partial \beta<0$. Higher R\&D spillovers reduce $\mathrm{R} \& \mathrm{D}$ expenditures but also the unit cost of production of all firms. The latter dominates the former:

$$
\left.\frac{\partial W}{\partial \beta}\right|_{\lambda=0}=\frac{1}{2} \frac{n(a-\bar{c})^{2} \gamma(n-1)}{[b \gamma(n+1)-1]^{2} B^{2}}>0 .
$$

In CE and for $\lambda=0, \partial q^{*} / \partial \beta=0$ and $\partial x^{*} / \partial \beta<0$. As in KMZ, welfare is increasing in $\beta$ :

$$
\left.\frac{\partial W}{\partial \beta}\right|_{\lambda=0}=\frac{n\left[\sigma\left(\frac{\alpha}{n}\right)^{\varepsilon} \kappa^{\varepsilon-1}\left(1-\frac{\varepsilon}{n}\right)\right]^{\frac{1}{\varepsilon-\alpha(1-\varepsilon)}}(n-1)}{B^{2}}>0 .
$$


Snapshot of the Application

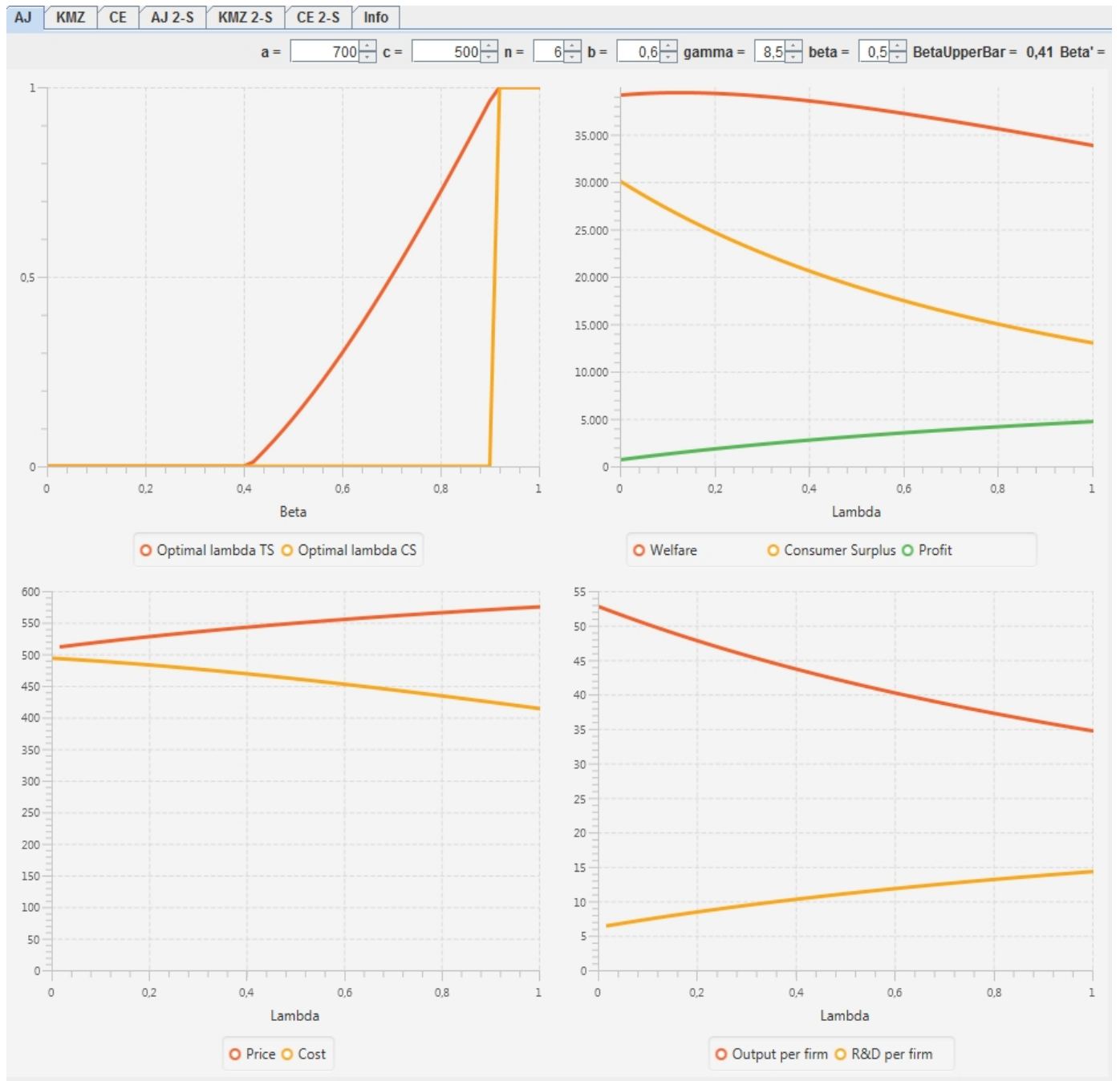

Fig. A5. AJ model. $(a=700, \bar{c}=500, \gamma=8.5, \beta=0.5, b=0.6)$ 
Optimal degree of overlapping ownership (TS and CS standard)

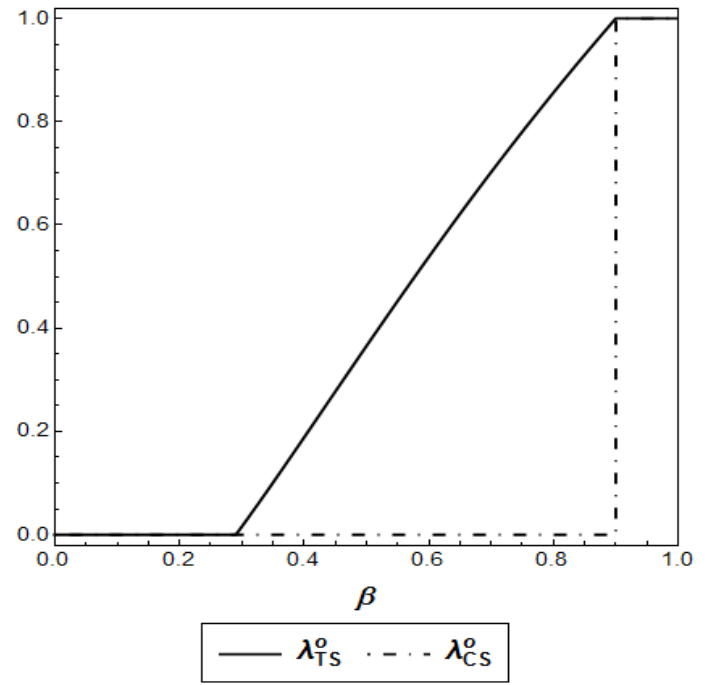

Fig. A6a. KMZ model.

$(\gamma=3, n=6, b=0.3)$

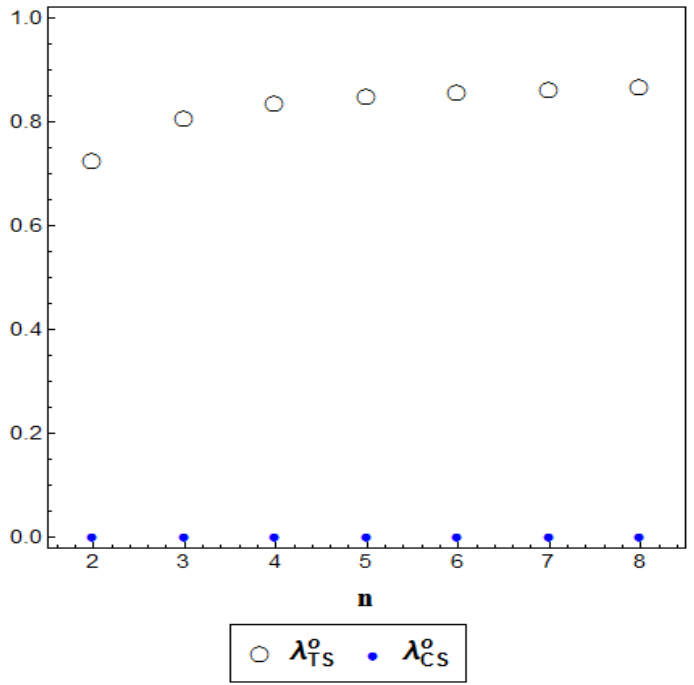

Fig. A6b. KMZ model.

$(\gamma=3, \beta=0.8, b=0.3)$

Table A5: Effect of Parameters on $\lambda_{\mathrm{TS}}^{o}$ and $\lambda_{\mathrm{CS}}^{o}$

\begin{tabular}{lccc||ccc}
\hline & \multicolumn{3}{c||}{$\lambda_{T S}^{o}$} & & $\lambda_{C S}^{o}$ & \\
& AJ & KMZ & CE & AJ & KMZ & CE \\
\hline Number of firms $(n)$ & + & + & + & $\langle+\rangle$ & 0 & $(+)$ \\
Elasticity of demand $\left(b^{-1}, \varepsilon^{-1}\right)$ & + & + & + & $\langle+\rangle$ & $\langle+\rangle$ & {$[+]$} \\
Elasticity of innovation function $\left(\gamma^{-1}, \alpha\right)$ & & + & + & $\langle+\rangle$ & $\langle+\rangle$ & {$[+]$} \\
Degree of spillover $(\beta)$ & + & + & + & $(+)$ & $(+)^{*}$ & {$[+]$} \\
\hline
\end{tabular}

Key: $\langle+\rangle$, the parameter enlarges the region where $\lambda_{\mathrm{CS}}^{o}=1 ;(+)$, the effect is positive only if both $\beta$ and $n$ are sufficiently large (otherwise there is no effect); $(+)^{*}$, the effect is positive only if the parameter is sufficiently large and $\gamma b$ is sufficiently small (otherwise there is no effect); $[+]$, the effect is positive when $n$ is sufficiently large (otherwise there is no effect).

\section{A.2.2 Two-stage model}

Next we present equilibrium values of output and $R \& D$ together with the expressions for $\operatorname{sign}\left\{\partial q^{*} / \partial \lambda\right\}$ and $\operatorname{sign}\left\{\partial x^{*} / \partial \lambda\right\}$ for each model specification. After that, we conduct a comparative statics analysis on $\bar{\beta}$, and on $\lambda_{\text {TS }}^{o}$ and $\lambda_{\text {CS }}^{o}$. Finally, we compare the static and the two-stage model and briefly discuss the comparative statics on the other parameters of the model.

Equilibrium values and $\operatorname{sign}\left\{\partial q^{*} / \partial \lambda\right\}$ and $\operatorname{sign}\left\{\partial x^{*} / \partial \lambda\right\}$. We consider each case in turn. 
Case AJ: FOCs $(10 ; 14)$ yield

$$
\begin{gathered}
-b \Lambda q^{*}+a-b n q^{*}-\bar{c}+B x^{*}=0 \\
{\left[\tau+\frac{\Lambda}{n+\Lambda}(n-1)(1+\lambda-2 \beta)\right] q^{*}-\gamma x^{*}=0 .}
\end{gathered}
$$

Solving the system for equilibrium values gives

$$
q^{*}=\frac{\gamma(a-\bar{c})}{\tilde{\Delta}} \text { and } x^{*}=\frac{\left[(n-1)\left(\frac{\Lambda}{n+\Lambda}\right)(1+\lambda-2 \beta)+\tau\right](a-\bar{c})}{\tilde{\Delta}}
$$

where

$$
\tilde{\Delta} \equiv \frac{\gamma b(\Lambda+n)^{2}-B[(n-1) \Lambda(1+\lambda-2 \beta)+(n+\Lambda) \tau]}{\Lambda+n} .
$$

In this case, as in the simultaneous model, $H(\beta)=b \gamma$, then using (16) we obtain

$$
\operatorname{sign}\left\{\frac{\partial q^{*}}{\partial \lambda}\right\}=\operatorname{sign}\left\{(B \beta-b \gamma)(n+\Lambda)+B\left[\frac{1+\lambda-2 \beta}{n+\Lambda}(n-1) n+\Lambda\right]\right\}
$$

and using (15) we get

$$
\begin{aligned}
\operatorname{sign}\left\{\frac{\partial x^{*}}{\partial \lambda}\right\}= & \operatorname{sign}\{\beta[\Lambda+n+(n-1)(\omega(\lambda)-\lambda)] \\
& \left.+\left[\frac{1+\lambda-2 \beta}{n+\Lambda}(n-1) n+\Lambda\right]-1-(n-1) \omega(\lambda) \tilde{\beta}(\lambda)\right\},
\end{aligned}
$$

where we have used that

$$
\left[\omega^{\prime}(\lambda)(\tilde{\beta}(\lambda)-\beta)+\omega(\lambda) \tilde{\beta}^{\prime}(\lambda)\right](\Lambda+n)=\frac{1+\lambda-2 \beta}{n+\Lambda}(n-1) n+\Lambda .
$$

Case KMZ: The output and $\mathrm{R} \& \mathrm{D}$ values in equilibrium are given by $(10 ; 14)$ :

$$
\begin{gathered}
-b \Lambda q^{*}+a-b n q^{*}-\bar{c}+\left[\left(\frac{2}{\gamma}\right) B x^{*}\right]^{1 / 2}=0 \\
\frac{1}{\gamma}\left[\left(\frac{2}{\gamma}\right) B x^{*}\right]^{-1 / 2}\left[\tau+(n-1) \frac{\Lambda}{n+\Lambda}(1+\lambda-2 \beta)\right] q^{*}-1=0 .
\end{gathered}
$$

Solving the system for equilibrium values gives

$$
q^{*}=\frac{\gamma(a-\bar{c})}{\gamma b(\Lambda+n)-\mu} \text { and } x^{*}=\frac{1}{2} \frac{(a-\bar{c})^{2} \vartheta_{K M Z}^{2} \gamma}{B[b \gamma(\Lambda+n)-\mu]^{2}}
$$

with $\vartheta_{K M Z} \equiv \tau+s(\lambda)(n-1)=(n-1) \frac{\Lambda}{n+\Lambda}(1+\lambda-2 \beta)+\tau$, where $s(\lambda) \equiv \omega(\lambda)(\tilde{\beta}(\lambda)-\beta)$. 
In this case, as in the simultaneous model, $H(\beta)=b \gamma B$, then from (16) we have

$$
\operatorname{sign}\left\{\frac{\partial q^{*}}{\partial \lambda}\right\}=\operatorname{sign}\left\{(\beta-b \gamma)(n+\Lambda)+\left[\frac{1+\lambda-2 \beta}{n+\Lambda}(n-1) n+\Lambda\right]\right\}
$$

and $\operatorname{sign}\left\{\frac{\partial x^{*}}{\partial \lambda}\right\}$ is again given by (58).

Case $C E$ : The output and $R \& D$ values in equilibrium are obtained from $(10 ; 14)$ :

$$
\begin{gathered}
\sigma Q^{*-\varepsilon}\left(1-\varepsilon \frac{\Lambda}{n}\right)-\kappa\left(B x^{*}\right)^{-\alpha}=0 \\
\alpha\left(B x^{*}\right)^{-\alpha-1}[\tau+(n-1) \omega(\lambda)(\tilde{\beta}(\lambda)-\beta)] \frac{Q^{*}}{n}=1 .
\end{gathered}
$$

Solving the system for $Q^{*}$ and $x^{*}$, after some manipulations, we get

$$
Q^{*}=\frac{n}{\alpha \kappa[(n-1) s(\lambda)+\tau]}\left(\sigma\left\{\frac{[(n-1) s(\lambda)+\tau] \alpha}{n}\right\}^{\varepsilon} \kappa^{\varepsilon-1}\left(1-\frac{\varepsilon \Lambda}{n}\right)\right)^{(1+\alpha) /[\varepsilon-\alpha(1-\varepsilon)]}
$$

and

$$
x^{*}=\frac{1}{B}\left(\sigma\left\{\frac{[(n-1) s(\lambda)+\tau] \alpha}{n}\right\}^{\varepsilon} \kappa^{\varepsilon-1}\left(1-\frac{\varepsilon \Lambda}{n}\right)\right)^{1 /[\varepsilon-\alpha(1-\varepsilon)]},
$$

where $s(\lambda) \equiv \omega(\lambda)(\tilde{\beta}(\lambda)-\beta)$ with

$$
\omega(\lambda)=\frac{\Lambda[2 n-\Lambda(1+\varepsilon)]}{n(n-\varepsilon \Lambda)} \quad \text { and } \quad \tilde{\beta}(\lambda)=\frac{n(1+\lambda)-\Lambda(1+\varepsilon)}{2 n-\Lambda(1+\varepsilon)} .
$$

Hence, we have

$$
\operatorname{sign}\left\{\frac{\partial q^{*}}{\partial \lambda}\right\}=\operatorname{sign}\left\{\left[\beta+s^{\prime}(\lambda)\right]-\frac{\alpha+1}{\alpha} \frac{\varepsilon}{n-\varepsilon \Lambda}[(n-1) s(\lambda)+\tau]\right\} .
$$

And, one can obtain $\operatorname{sign}\left\{\partial x^{*} / \partial \lambda\right\}$ by inserting values into (15) with $\delta=-(1+\varepsilon)$. 


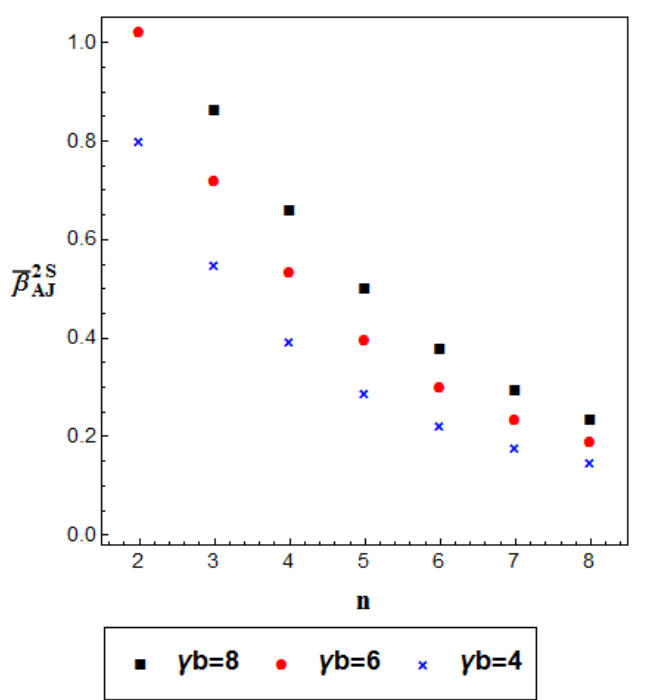

Fig. A7a. AJ model.

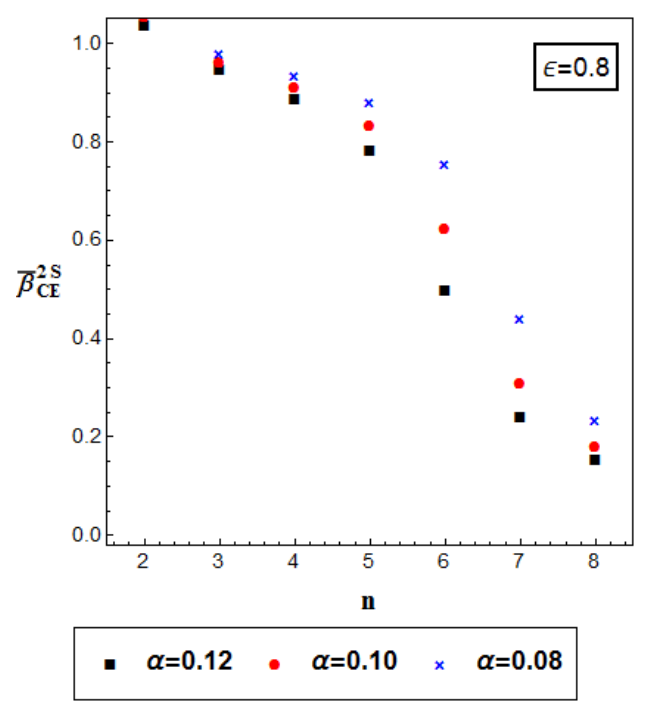

Fig. A8a. CE model.

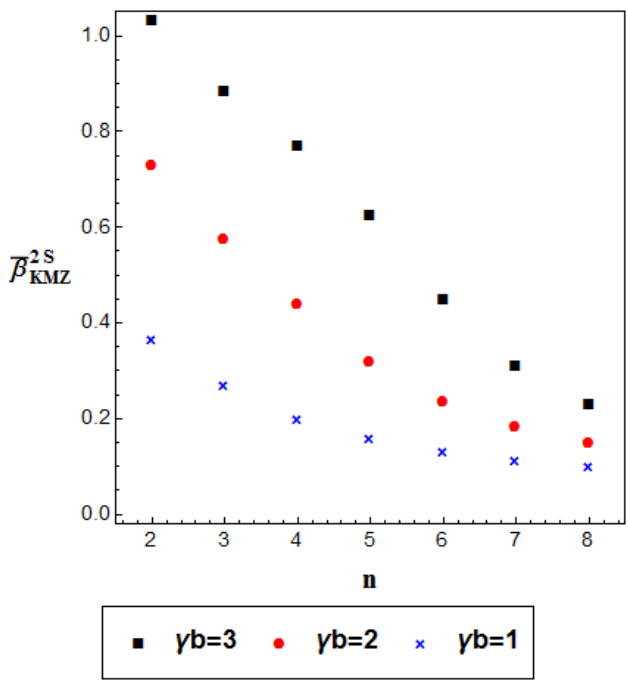

Fig. A7b. KMZ model.

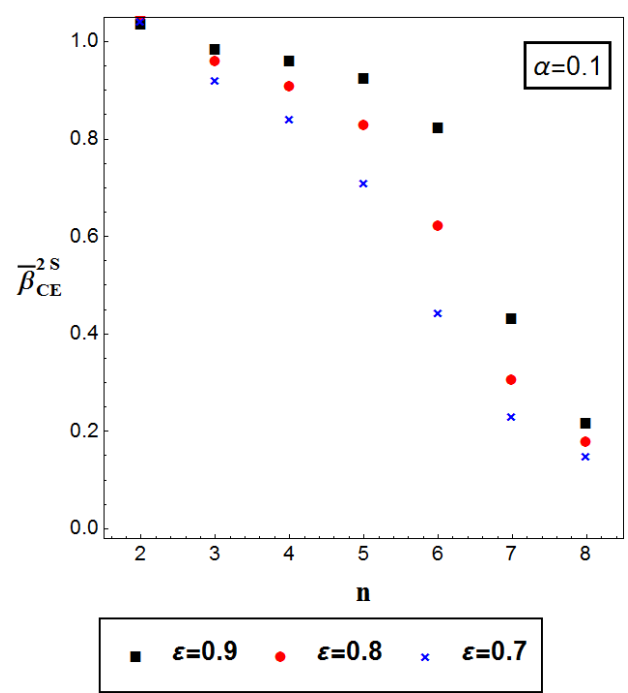

Fig. A8b. CE model.

Comparative statics on $\bar{\beta}$. Fig. A7a and A7b depict, respectively, the threshold $\bar{\beta}^{2 S}$ under the AJ and KMZ model specifications. Fig. A7b reveals that in $\mathrm{KMZ}, \bar{\beta}^{2 S}$ tends to be above 1 if we consider the same values as in AJ. In particular, only if $\gamma b$ is low enough, we have that $\bar{\beta}^{2 S}<1$ (this result is in line with the simultaneous model). Also, we observe that under the AJ and KMZ model specifications, $\bar{\beta}^{2 S}$ decreases with the number of firms and increases with $\gamma b$. Figures A8a (respectively A8b) depict the threshold $\bar{\beta}^{2 S}$ for the CE model for a given $\varepsilon(\alpha)$ and different values of $n$ and $\alpha(\varepsilon)$. As in the simultaneous model, the threshold value decreases with $n$, the elasticity of the innovation function, $\alpha$, and the elasticity of demand $\varepsilon^{-1}$. 
Comparative statics on $\lambda_{\mathrm{TS}}^{o}$ and $\lambda_{\mathrm{CS}}^{o}$. Fig. A9 is a snapshot of the application and plots welfare, consumer surplus, profit, price, cost, $q^{*}$ and $x^{*}$ as functions of $\lambda$ (for $\beta=0.65$ and $n=6)$. Fig. A10a, A10b and A10c show, respectively, optimal lambdas in AJ, KMZ and CE as functions of the number of firms. We see that under the three model specifications, $\lambda_{\text {TS }}^{o}$ weakly increases with $n$, whereas $\lambda_{\mathrm{CS}}^{o}$ jumps with $n$ only in AJ (and only for $n$ sufficiently large).

\section{Snapshot of the Application}

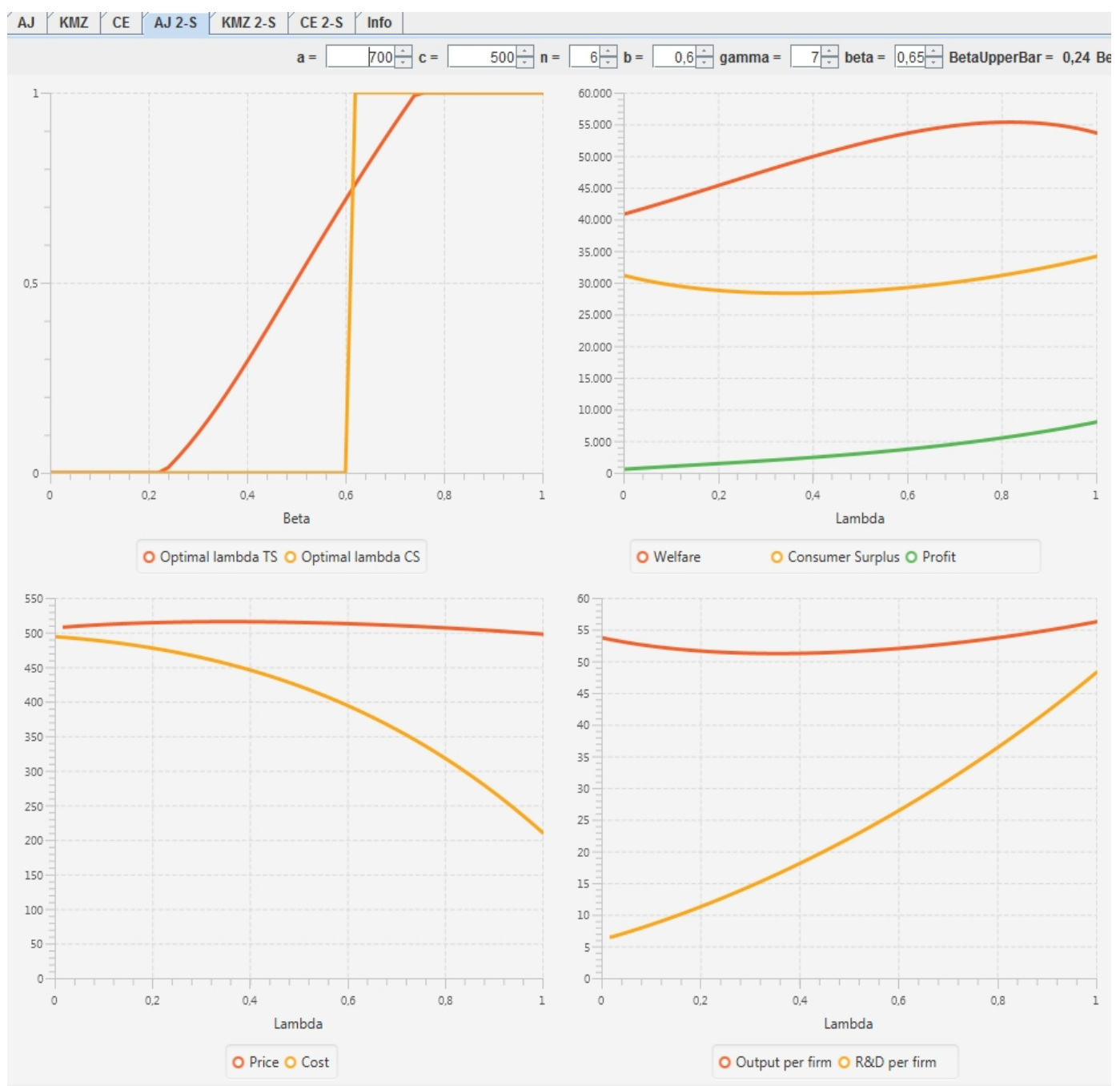

Fig. A9. AJ model. $(a=700, \bar{c}=500, \gamma=7, n=6, b=0.6)$ 
Optimal degree of overlapping ownership (TS and CS standard)

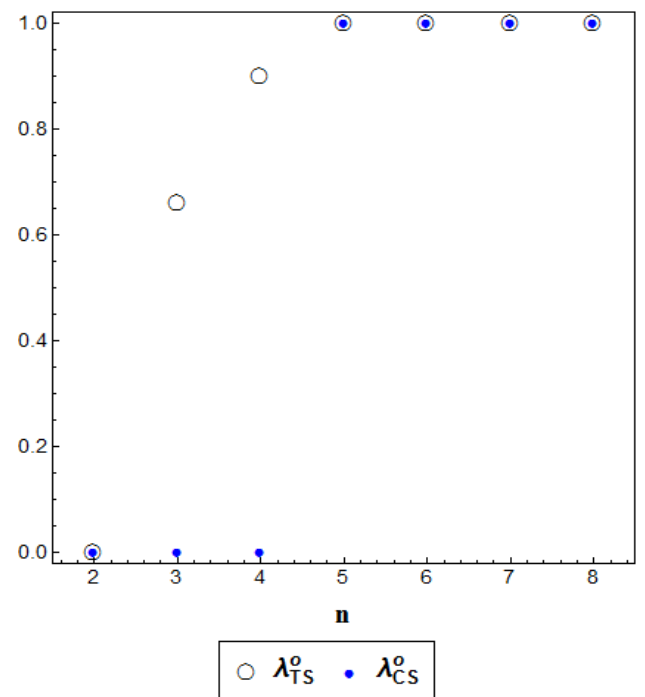

Fig. A10a. AJ model.

$$
\begin{gathered}
(a=700, \bar{c}=500, \gamma=7, \beta=0.8, \\
b=0.6)
\end{gathered}
$$

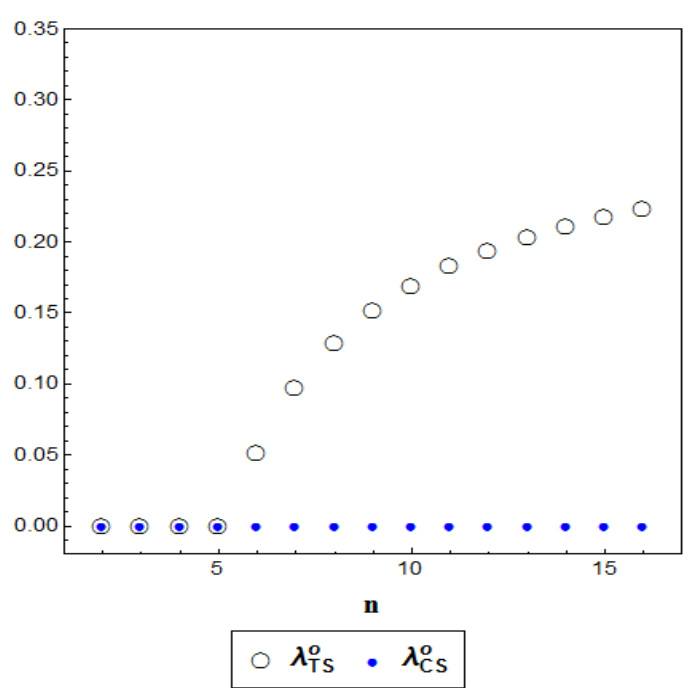

Fig. A10c. Constant elasticity model. $(\alpha=0.1, \varepsilon=0.8, \sigma=\kappa=1, \beta=0.8)$

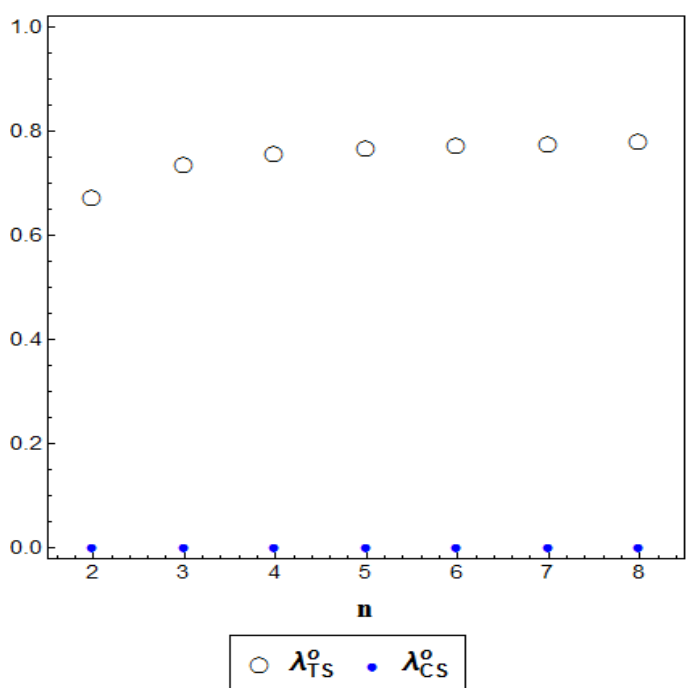

Fig. A10b. KMZ model. $(a=700, \bar{c}=500, \gamma=5, \beta=0.8$, $b=0.3)$

Comparison between the static and the two-stage model. In the constant elasticity model, as in the simultaneous case, we observe that if $n$ is small then the equilibrium is in $R_{\mathrm{I}}$, which implies that no overlapping ownership is socially optimal. Yet as $\beta$ and $n$ increase, $\lambda_{\mathrm{TS}}^{o}$ also increases. ${ }^{6}$ Note that $\lambda_{\mathrm{TS}}^{o}$ in the two-stage game is above the static level in a large region of

\footnotetext{
${ }^{6}$ This result is consistent with the literature. For example, in a model with no overlapping ownership Spence
} 
spillovers. For low values of $\beta$, the strategic effect is positive. Then, the two-stage model behaves differently than the static model in that welfare can increase with $\lambda$ in $R_{\mathrm{I}}$ because it reduces $\mathrm{R} \& \mathrm{D}$ overinvestment by firms. This case is illustrated in Figure A11, where - for low $\beta-\lambda_{\mathrm{TS}}^{o}$ in the two-stage model is larger than in the static model. For intermediate values of spillovers, the strategic effect becomes negative (but remains close to zero); for higher spillover values, $\lambda_{\mathrm{TS}}^{o}$ increases with $\beta$ more rapidly (i.e., convexly) when the strategic effect is strong.

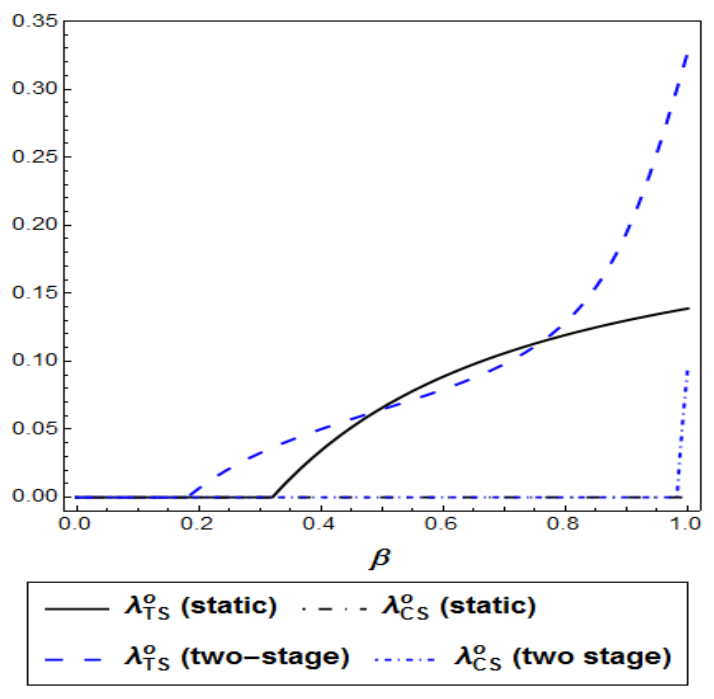

Fig. A11. Constant elasticity model. $(\alpha=0.1, \varepsilon=0.8, \sigma=\kappa=1, n=8$.

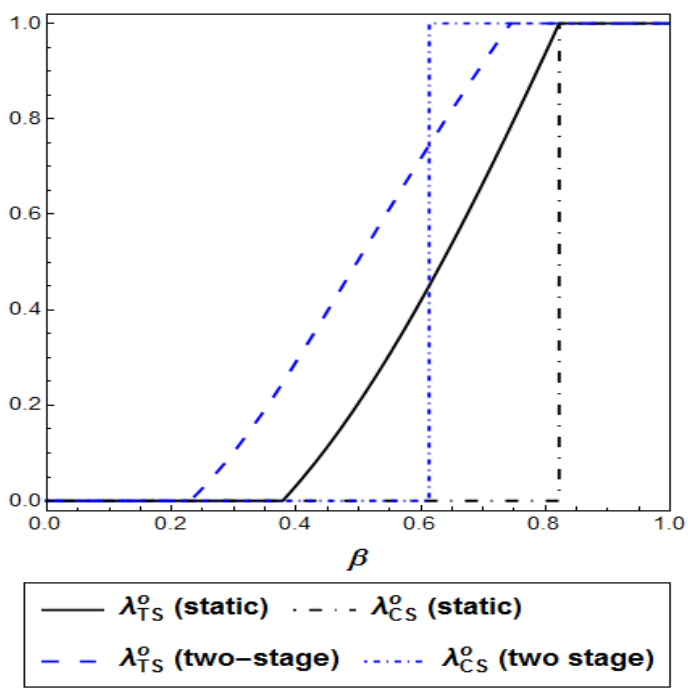

Fig A12. AJ model.

$$
(\gamma=7, n=6, b=0.6 .)
$$

In the AJ model, we find that $\lambda_{\mathrm{TS}}^{o}$ and $\lambda_{\mathrm{CS}}^{o}$ are weakly larger in the two-stage case (see Figure A12). In contrast with the static model, the simulations indicate (for $\beta=0.65$ and $n=6$ ) that prices may be hump-shaped while cost decreases with $\lambda$; correspondingly, output per firm is $U$-shaped when $R \& D$ per firm increases. The welfare translation of the increase in $\lambda$ displays U-shaped consumer surplus and increasing profit per firm, which results in an interior solution for welfare that features a large positive value of $\lambda_{\mathrm{TS}}^{o}$ (see Figure A9) with $\lambda_{\mathrm{CS}}^{o}=1>\lambda_{\mathrm{TS}}^{o}>0$.

This becomes possible when the strategic effect is positive and strong enough. Then there is overinvestment in $\mathrm{R} \& \mathrm{D}$ during the first stage, which boosts output in the second stage. The strategic effect becomes positive for intermediate values of $\beta$ when $\lambda$ is sufficiently high. For an intermediate level of spillovers, total surplus is not maximized with full cooperation because that would entail too much production (reducing firms' profits). ${ }^{7}$

(1984) used numerical simulations to demonstrate that an increase in $\beta$ reduces $x^{*}$ and that, for a given $\beta$ and $n \geq 2$, the cost reduction relative to the social optimum declines with $n$ (see Spence 1984, Table I). It is socially good then to increase the degree of profit internalization.

${ }^{7}$ More precisely, since $\beta^{2 S^{\prime}}$ decreases with $\lambda$, it follows that — for a given $\beta$ and a sufficiently high $\lambda$ - we have $\beta>\beta^{2 S \prime}$ and so the equilibrium is then in $R_{\mathrm{III}}$, where CS increases with $\lambda$ (CS is strictly convex in $\lambda$ and so 
Figure A13 shows optimal lambdas for KMZ as a function of $\beta$ in the simultaneous and two-stage model. As in AJ, we can have $\lambda_{\mathrm{CS}}^{o}>\lambda_{\mathrm{TS}}^{o}$ for intermediate spillover values (because of the strategic effect).

Optimal degree of overlapping ownership (TS and CS standard)

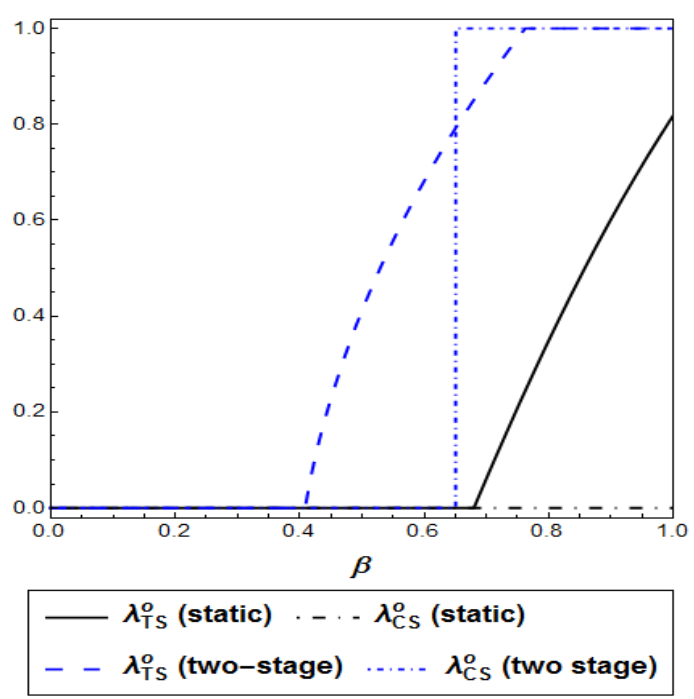

Fig. A13. KMZ model.

$$
(a=700, \bar{c}=500, \gamma=5.5, n=2,
$$

$$
b=0.2)
$$

The pattern of results in our comparative statics analysis of the other parameters in AJ, KMZ, and CE is similar to that for the one-stage game (see Table A5). The only exceptions we have found are as follows. In AJ: although decreasing $b$ enlarges the region where $\lambda_{\mathrm{CS}}^{o}=1$ is optimal (as in the static case), $\lambda_{\mathrm{CS}}^{o}$ can be lower than 1 (for a sufficiently low $b$ ) when spillovers are sufficiently high. In KMZ: although $\lambda_{\mathrm{CS}}^{o}$ is independent of $n$ in the static case, in the two-stage game it can decrease with $n$ when there are few firms in the market.

\section{B Bertrand competition with differentiated products}

\section{B.1 Framework and equilibrium}

In this Section we establish the framework and solve for the interior equilibrium of the model by deriving the FOCs.

$\lambda_{\mathrm{CS}}^{*}=1$ when $\left.\operatorname{CS}(1)>\operatorname{CS}(0)\right)$. In particular: for $\beta=0.62$, the equilibrium is in $R_{\mathrm{III}}$ when $\lambda>0.41$. Here the strategic effect is positive since $\tilde{\beta}(\lambda)>0.62$ for $\lambda>0.24$. Furthermore, if $\lambda>0.69$ then the strategic effect is strong enough to reverse the sign of the effect of $\partial x^{*} / \partial \lambda$ on $W^{\prime}(\lambda)$ (i.e., to make it negative); as a result, in a neighborhood of $\beta=0.62$ there is a global maximum for $W(\lambda)$ : even if the equilibrium is in $R_{\text {III }}$ we have that $W^{\prime}(\lambda)<0$ for high values of $\lambda$, which implies $\lambda_{\text {TS }}^{o} \in(0,1)$. 
We consider an industry with $n$ differentiated products, each produced by one firm. The demand for good $i$ is given by $q_{i}=D_{i}(\mathbf{p})$ where $\mathbf{p}$ is the vector of prices. Goods are (strict) gross substitutes, $\partial D_{i} / \partial p_{j}>0, j \neq i$. Assumptions A.2, A.3 and A.4 (with $H$ as defined below) are maintained, we replace Assumption A.1 by the following one:

Assumption 1B. For any product $i$, the function $D_{i}(\cdot)$ is smooth whenever positive, downward sloping, products are (strict) gross substitutes with $\partial D_{i} / \partial p_{j}>0, j \neq i$, and the demand system $D(\cdot)$ is symmetric with negative definite Jacobian.

Under Assumption 1B the demand system can be obtained from a representative consumer with quasilinear utility and can be inverted to obtain inverse demands (see Vives 1999, pp. 144148). Furthermore, it follows that the demand for a variety when all firms set the same price (the Chamberlinian DD function) is downward sloping since the own-price effect dominates the cross-price effects:

$$
v \equiv \frac{\partial D_{i}}{\partial p_{i}}+(n-1) \frac{\partial D_{j}}{\partial p_{i}}<0, j \neq i .
$$

It follows that $v_{\lambda} \equiv \partial D_{i} / \partial p_{i}+\lambda(n-1) \partial D_{k} / \partial p_{i}<0$. The innovation function is defined as in Cournot. The firm $i$ 's profit now writes as

$$
\pi_{i}=\left(p_{i}-c\left(x_{i}+\beta \sum_{j \neq i} x_{j}\right)\right) D_{i}(\mathbf{p})-\Gamma\left(x_{i}\right)
$$

and the objective function for the manager of firm $i$ is again: $\phi_{i}=\pi_{i}+\lambda \sum_{k \neq i} \pi_{k}$, thus

$\phi_{i}=\left(p_{i}-c\left(x_{i}+\beta \sum_{j \neq i} x_{j}\right)\right) D_{i}(\mathbf{p})-\Gamma\left(x_{i}\right)+\lambda \sum_{k \neq i}\left[\left(p_{k}-c\left(x_{k}+\beta \sum_{j \neq k} x_{j}\right)\right) D_{k}(\mathbf{p})-\Gamma\left(x_{k}\right)\right]$.

\section{B.2 Simultaneous model}

The FOCs for an interior symmetric equilibrium are

$$
\begin{gathered}
\frac{\partial \phi_{i}}{\partial p_{i}}=D_{i}(\mathbf{p})+\left(p_{i}-c_{i}\right) \frac{\partial D_{i}(\mathbf{p})}{\partial p_{i}}+\lambda \sum_{k \neq i}\left(p_{k}-c_{k}\right) \frac{\partial D_{k}(\mathbf{p})}{\partial p_{i}}=0, \\
\frac{\partial \phi_{i}}{\partial x_{i}}=-c^{\prime}(\cdot) D_{i}(\mathbf{p})-\Gamma^{\prime}\left(x_{i}\right)-\lambda \sum_{k \neq i} c^{\prime}(\cdot) \beta D_{k}(\mathbf{p})=0 .
\end{gathered}
$$

The symmetric equilibrium is the pair $\left(p^{*}, x^{*}\right)$, with $q^{*}=D_{i}\left(\mathbf{p}^{*}\right)$ where $\mathbf{p}^{*}=\left(p^{*}, \ldots, p^{*}\right)$ for all $i$, that solves the system (59)-(60). The FOC for price in the symmetric equilibrium is

$$
q^{*}+\left(p^{*}-c\left(B x^{*}\right)\right) \frac{\partial D_{i}\left(\mathbf{p}^{*}\right)}{\partial p_{i}}+\lambda(n-1)\left(p^{*}-c\left(B x^{*}\right)\right) \frac{\partial D_{k}\left(\mathbf{p}^{*}\right)}{\partial p_{i}}=0 .
$$


Note that $v<0$ ensures that $p^{*}-c\left(B x^{*}\right)$ is strictly positive for all $\lambda$; the above condition can be rewritten as

$$
q^{*}+\frac{\left(p^{*}-c\left(B x^{*}\right)\right)}{p^{*}} \frac{\partial D_{i}\left(\mathbf{p}^{*}\right)}{\partial p_{i}} \frac{p^{*} q^{*}}{D_{i}\left(\mathbf{p}^{*}\right)}+\lambda(n-1) \frac{\left(p^{*}-c\left(B x^{*}\right)\right)}{p^{*}} \frac{\partial D_{k}\left(\mathbf{p}^{*}\right)}{\partial p_{i}} \frac{p^{*} q^{*}}{D_{k}\left(\mathbf{p}^{*}\right)}=0 .
$$

Using the notation: $\eta_{i}=-\left(\partial D_{i}\left(\mathbf{p}^{*}\right) / \partial p_{i}\right)\left(p^{*} / D_{i}\left(\mathbf{p}^{*}\right)\right)$ and $\eta_{i k}=\left(\partial D_{k}\left(\mathbf{p}^{*}\right) / \partial p_{i}\right)\left(p^{*} / D_{k}\left(\mathbf{p}^{*}\right)\right)$, $k \neq i$, we can write

$$
1-\frac{p^{*}-c\left(B x^{*}\right)}{p^{*}} \eta_{i}+\lambda(n-1) \frac{p^{*}-c\left(B x^{*}\right)}{p^{*}} \eta_{i k}=0
$$

From the above condition and from (60), a symmetric (interior) equilibrium will satisfy the following two conditions:

$$
\begin{gathered}
\frac{p^{*}-c\left(B x^{*}\right)}{p^{*}}=\frac{1}{\eta_{i}-\lambda(n-1) \eta_{i k}} ; \\
-c^{\prime}\left(B x^{*}\right) q^{*} \tau=\Gamma^{\prime}\left(x^{*}\right) .
\end{gathered}
$$

Note that the latter condition is also obtained in Cournot oligopoly.

Finally, we assume the following parallel regularity conditions to the Cournot case:

$$
\Delta_{p} \equiv \partial_{p_{i} p_{i}} \phi_{i}+(n-1) \partial_{p_{i} p_{j}} \phi_{i}<0
$$

and

$$
\Delta \equiv \Delta_{p} \Delta_{x}-\left[\partial_{x_{i} p_{i}} \phi_{i}+(n-1) \partial_{p_{j} x_{i}} \phi_{i}\right]\left[\partial_{x_{i} p_{i}} \phi_{i}+(n-1) \partial_{x_{j} p_{i}} \phi_{i}\right]>0
$$

where

$$
\Delta_{x} \equiv \partial_{x_{i} x_{i}} \phi_{i}+(n-1) \partial_{x_{i} x_{j}} \phi_{i}
$$

Since $\partial_{x_{i} x_{i}} \phi_{i}=-c^{\prime \prime}\left(B x^{*}\right)\left[1+\lambda(n-1) \beta^{2}\right] q^{*}-\Gamma^{\prime \prime}\left(x^{*}\right)$ and $\partial_{x_{i} x_{j}} \phi_{i}=-c^{\prime \prime}\left(B x^{*}\right)[\tau+\lambda(1-\beta)] \beta q^{*}$, it follows that

$$
\Delta_{x}=-c^{\prime \prime}\left(B x^{*}\right) q^{*} \tau B-\Gamma^{\prime \prime}\left(x^{*}\right)<0
$$

under Assumptions A.2 and A.3. Together conditions (63) and (64) imply that the FOCs (61) and (62) both have a unique symmetric solution if they hold globally, and we assume that a symmetric regular equilibrium exists.

\section{B.2.1 Comparative statics with respect to $\lambda$}

In this Section we show that, as in the Cournot oligopoly model, if $\partial x^{*} / \partial \lambda \leq 0$, then $\partial p^{*} / \partial \lambda>0$ (Lemma B1). Secondly, we derive the signs: $\operatorname{sign}\left\{\partial x^{*} / \partial \lambda\right\}$ and sign $\left\{\partial p^{*} / \partial \lambda\right\}$ (Lemma B2). 
Finally, we discuss conditions that identify the three regions in Bertrand competition with product differentiation.

As in the Cournot oligopoly model, we can establish

LEMMA B1 In the symmetric equilibrium, $\frac{\partial p^{*}}{\partial \lambda}>0$ if $\frac{\partial x^{*}}{\partial \lambda} \leq 0$.

Proof. By totally differentiating the FOC $\partial \phi_{i} / \partial p_{i}=0$ with respect to $\lambda$ we obtain:

$$
\partial_{p_{i} p_{i}} \phi_{i} \frac{\partial p^{*}}{\partial \lambda}+(n-1) \partial_{p_{i} p_{j}} \phi_{i} \frac{\partial p^{*}}{\partial \lambda}+\partial_{x_{i} p_{i}} \phi_{i} \frac{\partial x^{*}}{\partial \lambda}+(n-1) \partial_{x_{j} p_{i}} \phi_{i} \frac{\partial x^{*}}{\partial \lambda}+\partial_{\lambda p_{i}} \phi_{i}=0 .
$$

Therefore,

$$
\frac{\partial p^{*}}{\partial \lambda}=-\frac{1}{\partial_{p_{i} p_{i}} \phi_{i}+(n-1) \partial_{p_{i} p_{j}} \phi_{i}}\left\{\partial_{\lambda p_{i}} \phi_{i}+\left[\partial_{x_{i} p_{i}} \phi_{i}+(n-1) \partial_{x_{j} p_{i}} \phi_{i}\right] \frac{\partial x^{*}}{\partial \lambda}\right\} .
$$

Using the stability condition $\Delta_{p}<0$, it follows that

$$
\operatorname{sign}\left\{\frac{\partial p^{*}}{\partial \lambda}\right\}=\operatorname{sign}\left\{\partial_{\lambda p_{i}} \phi_{i}+\left[\partial_{x_{i} p_{i}} \phi_{i}+(n-1) \partial_{x_{j} p_{i}} \phi_{i}\right] \frac{\partial x^{*}}{\partial \lambda}\right\}
$$

Since $\partial_{\lambda p_{i}} \phi_{i}=(n-1)\left(p^{*}-c\left(B x^{*}\right)\right) \partial D_{k}\left(\mathbf{p}^{*}\right) / \partial p_{i}>0$, we have that

$$
\frac{\partial x^{*}}{\partial \lambda} \leq 0 \Rightarrow \frac{\partial p^{*}}{\partial \lambda}>0 \text { when } \vartheta \equiv \partial_{x_{i} p_{i}} \phi_{i}+(n-1) \partial_{x_{j} p_{i}} \phi_{i}<0
$$

Note that

$$
\begin{aligned}
\partial_{x_{i} p_{i}} \phi_{i} & =-c^{\prime}\left(B x^{*}\right) \frac{\partial D_{i}\left(\mathbf{p}^{*}\right)}{\partial p_{i}}-\lambda(n-1) c^{\prime}\left(B x^{*}\right) \beta \frac{\partial D_{k}\left(\mathbf{p}^{*}\right)}{\partial p_{i}} \\
& =-\left[\frac{\partial D_{i}\left(\mathbf{p}^{*}\right)}{\partial p_{i}}+\lambda(n-1) \beta \frac{\partial D_{k}\left(\mathbf{p}^{*}\right)}{\partial p_{i}}\right] c^{\prime}\left(B x^{*}\right) .
\end{aligned}
$$

The expression $\partial_{x_{j} p_{i}} \phi_{i}$ can be obtained from (59):

$$
\begin{aligned}
\partial_{x_{j} p_{i}} \phi_{i} & =-c^{\prime}\left(B x^{*}\right) \beta \frac{\partial D_{i}\left(\mathbf{p}^{*}\right)}{\partial p_{i}}-\lambda c^{\prime}\left(B x^{*}\right) \frac{\partial D_{k}\left(\mathbf{p}^{*}\right)}{\partial p_{i}}-\lambda(n-2) c^{\prime}\left(B x^{*}\right) \beta \frac{\partial D_{k}\left(\mathbf{p}^{*}\right)}{\partial p_{i}} \\
& =-\left[\beta \frac{\partial D_{i}\left(\mathbf{p}^{*}\right)}{\partial p_{i}}+\lambda(B-\beta) \frac{\partial D_{k}\left(\mathbf{p}^{*}\right)}{\partial p_{i}}\right] c^{\prime}\left(B x^{*}\right) .
\end{aligned}
$$


Using the above expression we can write

$$
\begin{aligned}
\vartheta & =-\left\{\frac{\partial D_{i}\left(\mathbf{p}^{*}\right)}{\partial p_{i}}+\lambda(n-1) \beta \frac{\partial D_{k}\left(\mathbf{p}^{*}\right)}{\partial p_{i}}+(n-1)\left[\beta \frac{\partial D_{i}\left(\mathbf{p}^{*}\right)}{\partial p_{i}}+\lambda(B-\beta) \frac{\partial D_{k}\left(\mathbf{p}^{*}\right)}{\partial p_{i}}\right]\right\} c^{\prime}\left(B x^{*}\right) \\
& =-\left\{B \frac{\partial D_{i}\left(\mathbf{p}^{*}\right)}{\partial p_{i}}+(n-1)[\lambda \beta+\lambda(B-\beta)] \frac{\partial D_{k}\left(\mathbf{p}^{*}\right)}{\partial p_{i}}\right\} c^{\prime}\left(B x^{*}\right) \\
& =-\left[B \frac{\partial D_{i}\left(\mathbf{p}^{*}\right)}{\partial p_{i}}+\lambda(n-1) B \frac{\partial D_{k}\left(\mathbf{p}^{*}\right)}{\partial p_{i}}\right] c^{\prime}\left(B x^{*}\right) \\
& =-B\left[\frac{\partial D_{i}\left(\mathbf{p}^{*}\right)}{\partial p_{i}}+\lambda(n-1) \frac{\partial D_{k}\left(\mathbf{p}^{*}\right)}{\partial p_{i}}\right] c^{\prime}\left(B x^{*}\right) .
\end{aligned}
$$

Assumptions A.2 and $v<0$ imply that $\vartheta<0$

By totally differentiating the FOCs with respect to $\lambda$ and solving for $\partial p^{*} / \partial \lambda$ and $\partial x^{*} / \partial \lambda$ we obtain:

$$
\frac{\partial p^{*}}{\partial \lambda}=\frac{1}{\Delta}\left\{\partial_{\lambda x_{i}} \phi_{i}\left[\partial_{x_{i} p_{i}} \phi_{i}+(n-1) \partial_{x_{j} p_{i}} \phi_{i}\right]-\partial_{\lambda p_{i}} \phi_{i} \Delta_{x}\right\}
$$

and

$$
\frac{\partial x^{*}}{\partial \lambda}=\frac{1}{\Delta}\left\{\partial_{\lambda p_{i}} \phi_{i}\left[\partial_{x_{i} p_{i}} \phi_{i}+(n-1) \partial_{p_{j} x_{i}} \phi_{i}\right]-\partial_{\lambda x_{i}} \phi_{i} \Delta_{p}\right\}
$$

To obtain sign $\left\{\partial x^{*} / \partial \lambda\right\}$ and sign $\left\{\partial p^{*} / \partial \lambda\right\}$ we next derive in turn each of the expressions contained in equations (67) and (68). After some manipulations we can establish:

$$
\begin{gathered}
\partial_{x_{i} p_{i}} \phi_{i}+(n-1) \partial_{x_{j} p_{i}} \phi_{i}=-B v_{\lambda} c^{\prime}\left(B x^{*}\right), \\
\partial_{x_{i} p_{i}} \phi_{i}+(n-1) \partial_{p_{j} x_{i}} \phi_{i}=-\tau v c^{\prime}\left(B x^{*}\right) .
\end{gathered}
$$

We also have that

$$
\begin{gathered}
\partial_{\lambda x_{i}} \phi_{i}=-(n-1) c^{\prime}\left(B x^{*}\right) \beta q^{*} \geq 0, \\
\partial_{\lambda p_{i}} \phi_{i}=(n-1)\left(p^{*}-c\left(B x^{*}\right)\right) \frac{\partial D_{k}\left(\mathbf{p}^{*}\right)}{\partial p_{i}}>0 .
\end{gathered}
$$

Finally, we need the expressions for $\Delta_{p}$ (the expression for $\Delta_{x}$ is given by (65)). Recall that $\Delta_{p} \equiv \partial_{p_{i} p_{i}} \phi_{i}+(n-1) \partial_{p_{i} p_{j}} \phi_{i}$. By differentiating and evaluating in the symmetric equilibrium, we obtain

$$
\partial_{p_{i} p_{i}} \phi_{i}=2 \frac{\partial D_{i}\left(\mathbf{p}^{*}\right)}{\partial p_{i}}+\left(p^{*}-c\left(B x^{*}\right)\right)\left[\frac{\partial^{2} D_{i}\left(\mathbf{p}^{*}\right)}{\partial p_{i}^{2}}+\lambda(n-1) \frac{\partial^{2} D_{k}\left(\mathbf{p}^{*}\right)}{\partial p_{i}^{2}}\right]
$$

and, using that in the symmetric equilibrium $\partial D_{i} / \partial p_{j}=\partial D_{j} / \partial p_{i}$ and $\partial^{2} D_{i} / \partial p_{j} \partial p_{i}=\partial^{2} D_{j} / \partial p_{j} \partial p_{i}$,

$$
\partial_{p_{i} p_{j}} \phi_{i}=(1+\lambda) \frac{\partial D_{i}\left(\mathbf{p}^{*}\right)}{\partial p_{j}}+\left(p^{*}-c\left(B x^{*}\right)\right)\left[(1+\lambda) \frac{\partial^{2} D_{i}\left(\mathbf{p}^{*}\right)}{\partial p_{j} \partial p_{i}}+\lambda(n-2) \frac{\partial^{2} D_{k}\left(\mathbf{p}^{*}\right)}{\partial p_{j} \partial p_{i}}\right]
$$


Thus,

$$
\begin{aligned}
\Delta_{p}= & v+v_{\lambda}-\frac{q^{*}}{v_{\lambda}}(n-1)\left\{\frac{1}{n-1} \frac{\partial^{2} D_{i}\left(\mathbf{p}^{*}\right)}{\partial p_{i}^{2}}+\lambda \frac{\partial^{2} D_{k}\left(\mathbf{p}^{*}\right)}{\partial p_{i}^{2}}\right. \\
& \left.+\left[(1+\lambda) \frac{\partial^{2} D_{i}\left(\mathbf{p}^{*}\right)}{\partial p_{j} \partial p_{i}}+\lambda(n-2) \frac{\partial^{2} D_{k}\left(\mathbf{p}^{*}\right)}{\partial p_{j} \partial p_{i}}\right]\right\}
\end{aligned}
$$

Therefore,

$$
\Delta=-\Delta_{p}\left(c^{\prime \prime}\left(B x^{*}\right) q^{*} \tau B+\Gamma^{\prime \prime}\left(x^{*}\right)\right)-\tau B v v_{\lambda}\left(c^{\prime}\left(B x^{*}\right)\right)^{2} .
$$

Under regularity condition $\Delta>0$, then:

$$
\operatorname{sign}\left\{\frac{\partial x^{*}}{\partial \lambda}\right\}=\operatorname{sign}\left\{\tau\left(p^{*}-c\left(B x^{*}\right)\right) \frac{\partial D_{k}\left(\mathbf{p}^{*}\right)}{\partial p_{i}} v-\beta q^{*} \Delta_{p}\right\}
$$

and

$\operatorname{sign}\left\{\frac{\partial p^{*}}{\partial \lambda}\right\}=\operatorname{sign}\left\{-(n-1) c^{\prime}\left(B x^{*}\right) \beta q^{*}\left[-B v_{\lambda} c^{\prime}\left(B x^{*}\right)\right]-(n-1)\left(p^{*}-c\left(B x^{*}\right)\right) \frac{\partial D_{k}\left(\mathbf{p}^{*}\right)}{\partial p_{i}} \Delta_{x}\right\}$,

thus

$$
\operatorname{sign}\left\{\frac{\partial p^{*}}{\partial \lambda}\right\}=\operatorname{sign}\left\{-B \beta q^{*} v_{\lambda} c^{\prime}\left(B x^{*}\right)+\left(p^{*}-c\left(B x^{*}\right)\right) \frac{\partial D_{k}\left(\mathbf{p}^{*}\right)}{\partial p_{i}} \frac{\Delta_{x}}{c^{\prime}\left(B x^{*}\right)}\right\} .
$$

Clearly, from (71) and (72), and in line with the Cournot oligopoly model: for $\beta=0, \partial x^{*} / \partial \lambda<0$ and $\partial p^{*} / \partial \lambda>0$. Let $P^{\prime}(c)$ be the cost pass-through coefficient $P^{\prime}(c) \equiv d p^{*} / d c$; for $\beta>0$ we can establish the analogous to Lemmata 1 and 2:

LEMMA B2 In the symmetric equilibrium

$$
\operatorname{sign}\left\{\frac{\partial x^{*}}{\partial \lambda}\right\}=\operatorname{sign}\left\{\beta-P^{\prime}(c) \frac{|v|}{v_{\lambda}^{2}} \tau \frac{\partial D_{k}\left(\mathbf{p}^{*}\right)}{\partial p_{i}}\right\}
$$

where $P^{\prime}(c)=v_{\lambda} / \Delta_{p}>0$, and

$$
\operatorname{sign}\left\{\frac{\partial p^{*}}{\partial \lambda}\right\}=\operatorname{sign}\{H-\beta B\}
$$

where

$$
H=\frac{\partial D_{k}\left(\mathbf{p}^{*}\right) / \partial p_{i}}{\left(v_{\lambda} c^{\prime}\left(B x^{*}\right)\right)^{2}}\left[-\frac{c^{\prime \prime}\left(B x^{*}\right) B \Gamma^{\prime}\left(x^{*}\right)}{c^{\prime}\left(B x^{*}\right)}+\Gamma^{\prime \prime}\left(x^{*}\right)\right] .
$$

Proof. Inserting the FOC with respect to the price, $p^{*}-c\left(B x^{*}\right)=-q^{*} / v_{\lambda}$, into (71) yields

$$
\operatorname{sign}\left\{\partial x^{*} / \partial \lambda\right\}=\operatorname{sign}\left\{-\tau \frac{\partial D_{k}\left(\mathbf{p}^{*}\right)}{\partial p_{i}}\left(\frac{v}{v_{\lambda}}\right)-\beta \Delta_{p}\right\}
$$

By computing the total derivative of $\partial \phi_{i} / \partial p_{i}=0$ with respect to the $\operatorname{cost} c$, we obtain $P^{\prime}(c)=$ 
$v_{\lambda} / \Delta_{p}$, and therefore (73). Using again the FOC: $p^{*}-c\left(B x^{*}\right)=-q^{*} / v_{\lambda}$, and equation (65), we get

$$
\operatorname{sign}\left\{\frac{\partial p^{*}}{\partial \lambda}\right\}=\operatorname{sign}\left\{-B \beta v_{\lambda} c^{\prime}\left(B x^{*}\right)-\frac{1}{v_{\lambda} c^{\prime}\left(B x^{*}\right)} \frac{\partial D_{k}\left(\mathbf{p}^{*}\right)}{\partial p_{i}}\left[-c^{\prime \prime}\left(B x^{*}\right) q^{*}(\tau B)-\Gamma^{\prime \prime}\left(x^{*}\right)\right]\right\} .
$$

Noting that the FOC with respect to R\&D investment can be re-written as $q^{*}=\Gamma^{\prime}\left(x^{*}\right) /\left(-c^{\prime}\left(B x^{*}\right) \tau\right)$, and using that $v_{\lambda} c^{\prime}\left(B x^{*}\right)>0$, we have

$$
\operatorname{sign}\left\{\frac{\partial p^{*}}{\partial \lambda}\right\}=\operatorname{sign}\left\{-B+\frac{1}{\beta\left(v_{\lambda} c^{\prime}\left(B x^{*}\right)\right)^{2}} \frac{\partial D_{k}\left(\mathbf{p}^{*}\right)}{\partial p_{i}}\left[-\frac{c^{\prime \prime}\left(B x^{*}\right) B \Gamma^{\prime}\left(x^{*}\right)}{c^{\prime}\left(B x^{*}\right)}+\Gamma^{\prime \prime}\left(x^{*}\right)\right]\right\} \text {. }
$$

As in the Cournot oligopoly model we define the function $H$ for Bertrand competition with differentiated products as shown in equation (75). Thus, in the symmetric equilibrium: sign $\left\{\partial p^{*} / \partial \lambda\right\}=$ $\operatorname{sign}\{H-\beta B\}$

In Cournot we showed that sign $\left\{\partial q^{*} / \partial \lambda\right\}=\operatorname{sign}\{\beta B-H\}$. The reverse of the terms inside the curly brackets is explained by the different type of competition (price/output competition) in the two models. Assuming that $\Gamma^{\prime \prime}>0$, we can rewrite $H$ as follows:

$$
H=\frac{\partial D_{k}\left(\mathbf{p}^{*}\right) / \partial p_{i}}{\left(v_{\lambda} c^{\prime}\left(B x^{*}\right)\right)^{2}} \Gamma^{\prime \prime}\left(x^{*}\right)\left[-\frac{c^{\prime \prime}\left(B x^{*}\right) B x^{*}}{c^{\prime}\left(B x^{*}\right)} \frac{\Gamma^{\prime}\left(x^{*}\right)}{\Gamma^{\prime \prime}\left(x^{*}\right) x^{*}}+1\right]
$$

By defining, as we did in the Cournot model, $\chi\left(B x^{*}\right) \equiv-c^{\prime \prime}\left(B x^{*}\right) B x^{*} / c^{\prime}\left(B x^{*}\right) \geq 0, y\left(x^{*}\right) \equiv$ $\Gamma^{\prime \prime}\left(x^{*}\right) x^{*} / \Gamma^{\prime}\left(x^{*}\right) \geq 0$,

$$
\xi\left(q^{*}, x^{*}\right) \equiv \frac{\left(v_{\lambda} c^{\prime}\left(B x^{*}\right)\right)^{2}}{\frac{\partial D_{k}\left(p^{*}\right)}{\partial p_{i}} \Gamma^{\prime \prime}\left(x^{*}\right)}>0,
$$

and by replacing these terms into (76) we get

$$
H=\frac{1}{\xi\left(q^{*}, x^{*}\right)}\left(1+\frac{\chi\left(B x^{*}\right)}{y\left(x^{*}\right)}\right)
$$

Note that the only difference with respect to the Cournot model is that here the expression for the relative effectiveness of $\mathrm{R} \& \mathrm{D}(\xi)$ takes into account the fact that products are now differentiated. In Cournot: $\xi=-\left(c^{\prime}\left(B x^{*}\right)\right)^{2} /\left(f^{\prime}\left(Q^{*}\right) \Gamma^{\prime \prime}\left(x^{*}\right)\right)$; in Bertrand with differentiated products, however, the term $\left(f^{\prime}\right)^{-1}$ is replaced by $v_{\lambda}^{2}\left(\partial D_{k}\left(\mathbf{p}^{*}\right) / \partial p_{i}\right)^{-1}$.

We can proceed as in the Cournot model and define the corresponding three regions: $R_{\mathrm{I}}$, where $\partial p^{*} / \partial \lambda>0$ and $\partial x^{*} / \partial \lambda \leq 0 ; R_{\mathrm{II}}$ where $\partial p^{*} / \partial \lambda>0$ and $\partial x^{*} / \partial \lambda>0 ; R_{\text {III }}$ where $\partial p^{*} / \partial \lambda<0$ and $\partial x^{*} / \partial \lambda>0$.

Regarding $R_{\mathrm{I}}$, because of gross substitutes $\left(\partial D_{k}\left(\mathbf{p}^{*}\right) / \partial p_{i}>0\right)$, we can have $\partial x^{*} / \partial \lambda<0$ for all $\beta$ (73). This is the case when $-\Delta_{p}<\Lambda\left(\partial D_{k}\left(\mathbf{p}^{*}\right) / \partial p_{i}\right) v / v_{\lambda}$. Regarding the spillover 
threshold between $R_{\mathrm{II}}$ and $R_{\mathrm{III}}$, note that here, as in Cournot, Assumption A.4 implies that the equation $H-\beta B=0$ has a unique positive solution, which again we may denote by $\beta^{\prime}$. It follows that for $\beta>\beta^{\prime}, \partial p^{*} / \partial \lambda<0$. Furthermore, $R_{\text {III }}$ exists (because the threshold $\beta^{\prime}$ is strictly lower than 1) when $n>H(1)$.

\section{B.2.2 Welfare analysis}

Welfare (with quasilinear utility) at a symmetric equilibrium is given by

$$
W=U\left(\mathbf{q}^{*}\right)-c\left(B x^{*}\right) n q^{*}-n \Gamma\left(x^{*}\right)
$$

where $\mathbf{q}^{*}$ is the equilibrium output vector and $U$ is the utility of a representative consumer, assumed to be smooth and strictly concave (i.e., with a negative definite Hessian). By differentiating with respect to $\lambda$ :

$$
W^{\prime}(\lambda)=\left(\sum_{i} \frac{\partial U\left(\mathbf{q}^{*}\right)}{\partial q_{i}}-n c\left(B x^{*}\right)\right) \frac{\partial q^{*}}{\partial \lambda}-\left(n c^{\prime}\left(B x^{*}\right) B q^{*}+n \Gamma^{\prime}\left(x^{*}\right)\right) \frac{\partial x^{*}}{\partial \lambda}
$$

From the maximization problem of the consumer: $p_{i}=\partial U\left(\mathbf{q}^{*}\right) / \partial q_{i}$, so

$$
W^{\prime}(\lambda)=\left(p^{*}-c\left(B x^{*}\right)\right) n \frac{\partial q^{*}}{\partial \lambda}-\left(n c^{\prime}\left(B x^{*}\right) B q^{*}+n \Gamma^{\prime}\left(x^{*}\right)\right) \frac{\partial x^{*}}{\partial \lambda} .
$$

From the FOC with respect to price: $p^{*}-c\left(B x^{*}\right)=-q^{*} / v_{\lambda}$, and from the FOC with respect to R\&D investment: $\Gamma^{\prime}\left(x^{*}\right)=-c^{\prime}\left(B x^{*}\right) q^{*} \tau$, thus

$$
\begin{aligned}
W^{\prime}(\lambda) & =-\frac{q^{*}}{v_{\lambda}} n \frac{\partial q^{*}}{\partial \lambda}-\left(n c^{\prime}\left(B x^{*}\right) B q^{*}-n c^{\prime}\left(B x^{*}\right) q^{*} \tau\right) \frac{\partial x^{*}}{\partial \lambda} \\
& =-\frac{q^{*}}{v_{\lambda}} n \frac{\partial q^{*}}{\partial \lambda}-n c^{\prime}\left(B x^{*}\right) q^{*}(B-\tau) \frac{\partial x^{*}}{\partial \lambda} .
\end{aligned}
$$

From the demand definition, $q^{*}=D_{i}\left(p^{*}(\lambda)\right)$ we have that $\partial q^{*} / \partial \lambda=v\left(\partial p^{*} / \partial \lambda\right)$. Using that $B-\tau=(1-\lambda) \beta(n-1)$, we finally may write

$$
W^{\prime}(\lambda)=-\left[\frac{v}{v_{\lambda}} \frac{\partial p^{*}}{\partial \lambda}+(1-\lambda) \beta(n-1) c^{\prime}\left(B x^{*}\right) \frac{\partial x^{*}}{\partial \lambda}\right] n q^{*}
$$

Thus,

- in $R_{\mathrm{I}}$, where $\partial x^{*} / \partial \lambda \leq 0$ and $\partial p^{*} / \partial \lambda>0$ (so $\partial q^{*} / \partial \lambda<0$ ): $W^{\prime}(\lambda)<0$.

- in $R_{\mathrm{II}}$, where $\partial x^{*} / \partial \lambda>0$ and $\partial p^{*} / \partial \lambda>0$ (so $\left.\partial q^{*} / \partial \lambda<0\right): W^{\prime}(\lambda) \lessgtr 0$.

- in $R_{\mathrm{III}}$, where $\partial x^{*} / \partial \lambda>0$ and $\partial p^{*} / \partial \lambda<0\left(\right.$ so $\left.\partial q^{*} / \partial \lambda>0\right): W^{\prime}(\lambda)>0$. 
From (67), it follows that

$$
\frac{\partial p^{*}}{\partial \lambda}=\frac{(n-1) q^{*}}{\Delta}\left\{\left(c^{\prime}\left(B x^{*}\right)\right)^{2} \beta B v_{\lambda}-\frac{\partial D_{k}\left(\mathbf{p}^{*}\right) / \partial p_{i}}{v_{\lambda}}\left[c^{\prime \prime}\left(B x^{*}\right) q^{*} B \tau+\Gamma^{\prime \prime}\left(x^{*}\right)\right]\right\} .
$$

Similarly, from (68), after some manipulations, we obtain

$$
\frac{\partial x^{*}}{\partial \lambda}=\frac{(n-1) q^{*}\left(-c^{\prime}\left(B x^{*}\right)\right)}{\Delta} \frac{\partial D_{k}\left(\mathbf{p}^{*}\right)}{\partial p_{i}}\left[\beta\left(\frac{\Delta_{p}}{-\frac{\partial D_{k}\left(\mathbf{p}^{*}\right)}{\partial p_{i}}}\right)-\frac{v}{v_{\lambda}} \tau\right]
$$

By inserting (78) and (79) into (77) we obtain

$$
W^{\prime}(\lambda)=\frac{(n-1) n q^{* 2}}{\Delta} \frac{\partial D_{k}\left(\mathbf{p}^{*}\right)}{\partial p_{i}} \digamma
$$

where

$$
\begin{aligned}
\digamma \equiv & \frac{\left(c^{\prime}\left(B x^{*}\right)\right)^{2} \beta B(-v)}{\partial D_{k}\left(\mathbf{p}^{*}\right) / \partial p_{i}}+\frac{v}{v_{\lambda}^{2}}\left[c^{\prime \prime}\left(B x^{*}\right) q^{*} B \tau+\Gamma^{\prime \prime}\left(x^{*}\right)\right] \\
& +\left(c^{\prime}\left(B x^{*}\right)\right)^{2}(1-\lambda) \beta(n-1)\left[\frac{\beta\left(-\Delta_{p}\right)}{\partial D_{k}\left(\mathbf{p}^{*}\right) / \partial p_{i}}-\frac{v}{v_{\lambda}} \tau\right] .
\end{aligned}
$$

Remark B1. Consider the case of independent products, $\partial D_{k}\left(\mathbf{p}^{*}\right) / \partial p_{i}=0$. If the local monopoly problem is well-defined we have: (i) if $\beta>0$, then $\lambda_{\mathrm{TS}}^{o}=\lambda_{\mathrm{CS}}^{o}=1$, whereas (ii) if $\beta=0$, then $\lambda$ has no impact on total surplus or consumer surplus.

Proof. It follows immediately from equation (71) that $\operatorname{sign}\left\{\partial x^{*} / \partial \lambda\right\}>0$ for $\beta>0$ and $\partial x^{*} / \partial \lambda=0$ for $\beta=0$. Similarly, from equation (72): $\operatorname{sign}\left\{\partial p^{*} / \partial \lambda\right\}<0$ (or equivalently $\left.\operatorname{sign}\left\{\partial q^{*} / \partial \lambda\right\}>0\right)$ for $\beta>0$, while $\partial p^{*} / \partial \lambda=\partial q^{*} / \partial \lambda=0$ for $\beta=0$. Using $(77), W^{\prime}(\lambda)>0$ for all $\lambda$ if $\beta>0$, thus $\lambda_{\text {TS }}^{o}=1$. Since sign $\left\{C S^{\prime}(\lambda)\right\}=\operatorname{sign}\left\{\partial q^{*} / \partial \lambda\right\}$, we also have that $\lambda_{\mathrm{CS}}^{o}=1$. If $\beta=0$, clearly from (77), $W^{\prime}(\lambda)=0$; note that for $\partial D_{k}\left(\mathbf{p}^{*}\right) / \partial p_{i}=\beta=0$, FOCs do not depend on $\lambda$

\section{B.3 Two-stage model}

We first derive the FOCs and the expression for $\tilde{\beta}(\lambda)$ for the Bertrand case. We then discuss the strategic effect and welfare in Bertrand with two stages.

Interior equilibrium and threshold $\tilde{\beta}(\lambda)$. Let

$$
\varphi \equiv-\frac{\partial D_{i}\left(\mathbf{p}^{*}\right)}{\partial p_{i}} \partial_{p_{i} p_{i}} \phi_{i}+\lambda \frac{\partial D_{k}\left(\mathbf{p}^{*}\right)}{\partial p_{i}}\left[(n-1) \partial_{p_{i} p_{j}} \phi_{i}-(n-2) \partial_{p_{i} p_{i}} \phi_{i}\right]
$$


Then, using (36) we can write:

$$
\frac{\partial}{\partial x_{i}} p_{j}^{*}(\mathbf{x})=\frac{-c^{\prime}(B x)}{\Omega}(-\varphi)(\tilde{\beta}(\lambda)-\beta)
$$

where

$$
\Omega \equiv\left(\partial_{p_{i} p_{i}} \phi_{i}-\partial_{p_{i} p_{j}} \phi_{i}\right)\left[\partial_{p_{i} p_{i}} \phi_{i}+(n-1) \partial_{p_{i} p_{j}} \phi_{i}\right]
$$

and

$$
\tilde{\beta}(\lambda)=\frac{1}{(-\varphi)}\left[\frac{\partial D_{i}\left(\mathbf{p}^{*}\right)}{\partial p_{i}} \partial_{p_{i} p_{j}} \phi_{i}-\lambda \frac{\partial D_{j}\left(\mathbf{p}^{*}\right)}{\partial p_{i}} \partial_{p_{i} p_{i}} \phi_{i}\right] .
$$

The denominator of $\tilde{\beta}(\lambda)$ is positive since $\varphi<0$ :

$$
\begin{aligned}
\varphi & \equiv-\frac{\partial D_{i}\left(\mathbf{p}^{*}\right)}{\partial p_{i}} \partial_{p_{i} p_{i}} \phi_{i}+\lambda \frac{\partial D_{k}\left(\mathbf{p}^{*}\right)}{\partial p_{i}}\left[(n-1) \partial_{p_{i} p_{j}} \phi_{i}-(n-2) \partial_{p_{i} p_{i}} \phi_{i}\right] \\
& =-\frac{\partial D_{i}\left(\mathbf{p}^{*}\right)}{\partial p_{i}} \partial_{p_{i} p_{i}} \phi_{i}+\lambda \frac{\partial D_{k}\left(\mathbf{p}^{*}\right)}{\partial p_{i}}\left[\partial_{p_{i} p_{i}} \phi_{i}+(n-1) \partial_{p_{i} p_{j}} \phi_{i}\right]-\lambda \frac{\partial D_{k}\left(\mathbf{p}^{*}\right)}{\partial p_{i}}(n-1) \partial_{p_{i} p_{i}} \phi_{i} \\
& =\lambda \frac{\partial D_{k}\left(\mathbf{p}^{*}\right)}{\partial p_{i}} \Delta_{p}-\partial_{p_{i} p_{i}} \phi_{i}\left[\frac{\partial D_{i}\left(\mathbf{p}^{*}\right)}{\partial p_{i}}+\lambda(n-1) \frac{\partial D_{k}\left(\mathbf{p}^{*}\right)}{\partial p_{i}}\right]<0 .
\end{aligned}
$$

Therefore, if

$$
\frac{\partial D_{i}\left(\mathbf{p}^{*}\right)}{\partial p_{i}} \partial_{p_{i} p_{j}} \phi_{i}-\lambda \frac{\partial D_{j}\left(\mathbf{p}^{*}\right)}{\partial p_{i}} \partial_{p_{i} p_{i}} \phi_{i}<0
$$

then $\tilde{\beta}(\lambda)<0$. Condition (86) is satisfied in the case of linear and constant elasticity demand with differentiated products (see analysis below).

Finally, note that in Bertrand at the symmetric equilibrium FOCs boil down to

$$
q^{*}+\left(p^{*}-c\left(B x^{*}\right)\right) v_{\lambda}=0
$$

and

$$
-c^{\prime}\left(B x^{*}\right) \tau q^{*}-\Gamma^{\prime}\left(x^{*}\right)+(n-1) \frac{\partial \phi_{i}}{\partial p_{j}}\left(\frac{\partial p_{j}^{*}}{\partial x_{i}}\right)=0 .
$$

Strategic effect. The strategic effect is

$$
\psi(\mathbf{x}) \equiv(n-1) \frac{\partial}{\partial p_{j}} \phi_{i}\left(\mathbf{p}^{*}(\mathbf{x}), \mathbf{x}\right) \frac{\partial}{\partial x_{i}} p_{j}^{*}(\mathbf{x})
$$

Next we show that $\partial \phi_{i} / \partial p_{j}$ is strictly positive for $\beta<1$. We then show that $\partial p_{j}^{*} / \partial x_{i}<0$ with strategic complements price competition and $\beta$ high enough, and as a result the strategic effect is negative. 
We can write the FOC with respect to $R \& D$ as

$$
\frac{\partial}{\partial x_{i}} \phi_{i}\left(\mathbf{p}^{*}(\mathbf{x}), \mathbf{x}, \lambda\right)+(n-1) \frac{\partial}{\partial p_{j}} \phi_{i}\left(\mathbf{p}^{*}(\mathbf{x}), \mathbf{x}, \lambda\right) \frac{\partial}{\partial x_{i}} p_{j}^{*}(\mathbf{x})=0
$$

and

$$
\begin{aligned}
\frac{\partial}{\partial p_{j}} \phi_{i}\left(\mathbf{p}^{*}(\mathbf{x}), \mathbf{x}, \lambda\right)= & \left(p^{*}-c(B x)\right) \frac{\partial D_{i}\left(\mathbf{p}^{*}\right)}{\partial p_{j}}+\lambda\left[q^{*}+\left(p^{*}-c(B x)\right) \frac{\partial D_{j}\left(\mathbf{p}^{*}\right)}{\partial p_{j}}\right] \\
& +\lambda(n-2)\left(p^{*}-c(B x)\right) \frac{\partial D_{k}\left(\mathbf{p}^{*}\right)}{\partial p_{j}}
\end{aligned}
$$

which can be rewritten as

$$
\frac{\partial}{\partial p_{j}} \phi_{i}\left(\mathbf{p}^{*}(\mathbf{x}), \mathbf{x}, \lambda\right)=\frac{-q^{*}}{v_{\lambda}}\left[\frac{\partial D_{i}\left(\mathbf{p}^{*}\right)}{\partial p_{j}}+\lambda \frac{\partial D_{j}\left(\mathbf{p}^{*}\right)}{\partial p_{j}}+\lambda(n-2) \frac{\partial D_{k}\left(\mathbf{p}^{*}\right)}{\partial p_{j}}\right]+\lambda q^{*}
$$

where we have used the FOC: $\left(p^{*}-c(B x)\right)=-q^{*} / v_{\lambda}$. To show that $\partial \phi_{i}\left(\mathbf{p}^{*}(\mathbf{x}), \mathbf{x}, \lambda\right) / \partial p_{j}>0$, we rewrite (90) as follows:

$$
\begin{aligned}
\frac{\partial}{\partial p_{j}} \phi_{i}\left(\mathbf{p}^{*}(\mathbf{x}), \mathbf{x}, \lambda\right)= & \frac{-q^{*}}{v_{\lambda}}\left[\frac{\partial D_{i}\left(\mathbf{p}^{*}\right)}{\partial p_{j}}+\lambda \frac{\partial D_{j}\left(\mathbf{p}^{*}\right)}{\partial p_{j}}+\lambda(n-2) \frac{\partial D_{k}\left(\mathbf{p}^{*}\right)}{\partial p_{j}}-\lambda v_{\lambda}\right] \\
= & \frac{-q^{*}}{v_{\lambda}}\left[\frac{\partial D_{i}\left(\mathbf{p}^{*}\right)}{\partial p_{j}}+\lambda \frac{\partial D_{j}\left(\mathbf{p}^{*}\right)}{\partial p_{j}}+\lambda(n-2) \frac{\partial D_{k}\left(\mathbf{p}^{*}\right)}{\partial p_{j}}\right. \\
& \left.-\lambda\left(\frac{\partial D_{i}\left(\mathbf{p}^{*}\right)}{\partial p_{i}}+\lambda(n-1) \frac{\partial D_{k}\left(\mathbf{p}^{*}\right)}{\partial p_{i}}\right)\right] .
\end{aligned}
$$

Using now that in the symmetric equilibrium $\partial D_{i} / \partial p_{i}=\partial D_{j} / \partial p_{j}$ and $\partial D_{i} / \partial p_{j}=\partial D_{k} / \partial p_{j}=$ $\partial D_{k} / \partial p_{i}$ for $i \neq j, j \neq k, i \neq k$ we can rewrite the above expression as follows

$$
\begin{aligned}
\frac{\partial}{\partial p_{j}} \phi_{i}\left(\mathbf{p}^{*}(\mathbf{x}), \mathbf{x}, \lambda\right) & =\frac{-q^{*}}{v_{\lambda}}\left[1+\lambda(n-2)-\lambda^{2}(n-1)\right] \frac{\partial D_{i}\left(\mathbf{p}^{*}\right)}{\partial p_{j}} \\
& =\frac{-q^{*}}{v_{\lambda}}(1-\lambda) \Lambda \frac{\partial D_{i}\left(\mathbf{p}^{*}\right)}{\partial p_{j}}>0 \text { for } \lambda<1 .
\end{aligned}
$$

We now show that $\partial^{2} \phi_{i} / \partial x_{j} \partial p_{i}$ is negative or positive depending on whether $\beta$ is high or low. Note that:

$$
\frac{\partial^{2} \phi_{i}}{\partial x_{i} \partial p_{i}}(\mathbf{x})=-c^{\prime}(B x)\left[\frac{\partial D_{i}\left(\mathbf{p}^{*}\right)}{\partial p_{i}}+\lambda(n-1) \beta \frac{\partial D_{k}\left(\mathbf{p}^{*}\right)}{\partial p_{i}}\right]
$$


and

$$
\begin{aligned}
\frac{\partial^{2} \phi_{i}}{\partial x_{j} \partial p_{i}}(\mathbf{x}) & =-c^{\prime}(B x)\left[\beta \frac{\partial D_{i}\left(\mathbf{p}^{*}\right)}{\partial p_{i}}+\lambda \frac{\partial D_{j}\left(\mathbf{p}^{*}\right)}{\partial p_{i}}+\lambda(n-2) \beta \frac{\partial D_{k}\left(\mathbf{p}^{*}\right)}{\partial p_{i}}\right] \\
& =-c^{\prime}(B x)\left\{\beta\left[\frac{\partial D_{i}\left(\mathbf{p}^{*}\right)}{\partial p_{i}}+\lambda(n-1) \beta \frac{\partial D_{k}\left(\mathbf{p}^{*}\right)}{\partial p_{i}}\right]+(1-\beta) \lambda \frac{\partial D_{k}\left(\mathbf{p}^{*}\right)}{\partial p_{i}}\right\}
\end{aligned}
$$

Therefore, $\partial^{2} \phi_{i} / \partial x_{j} \partial p_{i}<0$ for $\beta$ high enough. From (36), we have

$$
\frac{\partial}{\partial x_{i}} p_{j}^{*}(\mathbf{x})=\frac{1}{\Omega}\left(\partial_{x_{i} p_{i}} \phi_{i} \partial_{p_{i} p_{j}} \phi_{i}-\partial_{x_{h} p_{i}} \phi_{i} \partial_{p_{i} p_{i}} \phi_{i}\right),
$$

where in the symmetric equilibrium, and using that $p^{*}-c(B x)=-q^{*} / v_{\lambda}$,

$$
\partial_{p_{i} p_{i}} \phi_{i}(\mathbf{x})=2 \frac{\partial D_{i}\left(\mathbf{p}^{*}\right)}{\partial p_{i}}+\left(\frac{-q^{*}}{v_{\lambda}}\right)\left[\frac{\partial^{2} D_{i}\left(\mathbf{p}^{*}\right)}{\left(\partial p_{i}\right)^{2}}+\lambda(n-1) \frac{\partial^{2} D_{k}\left(\mathbf{p}^{*}\right)}{\left(\partial p_{i}\right)^{2}}\right]
$$

and

$$
\partial_{p_{i} p_{j}} \phi_{i}(\mathbf{x})=\frac{\partial D_{i}\left(\mathbf{p}^{*}\right)}{\partial p_{j}}+\left(\frac{-q^{*}}{v_{\lambda}}\right)\left[(1+\lambda) \frac{\partial^{2} D_{i}\left(\mathbf{p}^{*}\right)}{\partial p_{j} \partial p_{i}}+\lambda(n-2) \frac{\partial^{2} D_{k}\left(\mathbf{p}^{*}\right)}{\partial p_{j} \partial p_{i}}\right]+\lambda \frac{\partial D_{j}\left(\mathbf{p}^{*}\right)}{\partial p_{i}} .
$$

Strategic complements price competition $\partial_{p_{i} p_{j}} \phi_{i}(\mathbf{x})>0$, together with the assumption $\Delta_{p}<$ 0 , both imply that $\Omega>0$. Note also that the assumption $v<0$ implies $\partial^{2} \phi_{i} / \partial x_{i} \partial p_{i}<0$, and since the expression for $\partial^{2} \phi_{i} / \partial x_{j} \partial p_{i}$ becomes negative for $\beta$ high enough, we can establish:

$$
\frac{\partial p_{j}^{*}}{\partial x_{i}}<0 \text { with strategic complements price competition and } \beta \text { high enough, }
$$

in which case the strategic effect is negative and firms adopt a "puppy dog" strategy (Fudenberg and Tirole 1984): increasing $x_{i}$ decreases the prices of rivals because a larger $x_{i}$ shifts the price best reply of firm $j$ inwards as $\partial^{2} \phi_{j} / \partial x_{i} \partial p_{j}<0$ and also shift inwards the price best reply of firm $i$ since $\partial^{2} \phi_{i} / \partial x_{i} \partial p_{i}<0$. The result is that the strategic effect is negative $(\psi<0)$ and we have puppy dog investment incentives.

Welfare. From our previous analysis:

$$
W^{\prime}(\lambda)=\left(p^{*}-c\left(B x^{*}\right)\right) n \frac{\partial q^{*}}{\partial \lambda}-\left(n c^{\prime}\left(B x^{*}\right) B q^{*}+n \Gamma^{\prime}\left(x^{*}\right)\right) \frac{\partial x^{*}}{\partial \lambda} .
$$

The FOC with respect to $x$ is

$$
\Gamma^{\prime}\left(x^{*}\right)=-c^{\prime}\left(B x^{*}\right)[\tau+(n-1) \omega(\lambda)(\tilde{\beta}(\lambda)-\beta)] q^{*} .
$$


Inserting the FOCs $p^{*}-c\left(B x^{*}\right)=-q^{*} / v_{\lambda}$ and (93) into the expression for $W^{\prime}(\lambda)$ we obtain:

$$
W^{\prime}(\lambda)=\left\{-\frac{1}{v_{\lambda}} \frac{\partial q^{*}}{\partial \lambda}-[(1-\lambda) \beta-\omega(\lambda)(\tilde{\beta}(\lambda)-\beta)](n-1) c^{\prime}\left(B x^{*}\right) \frac{\partial x^{*}}{\partial \lambda}\right\} n q^{*}
$$

In Cournot when the strategic effect is negative (i.e., $(\tilde{\beta}(\lambda)-\beta)<0$ ), the sign of the impact of $\lambda$ on welfare in each region $\left(R_{\mathrm{I}}, R_{\mathrm{II}}\right.$ and $\left.R_{\mathrm{III}}\right)$ is the same in the simultaneous and the two-stage model. This is the case also with Bertrand competition and $\beta$ high (puppy dog strategy).

\section{B.4 Model specifications}

In this section we characterize the model with linear and constant elasticity demands analogs to AJ and CE. For each case, we first consider the simultaneous and then the two-stage model.

\section{B.4.1 Linear model}

Model specification: main assumptions. We assume the following: $D_{i}(p)=a-b p_{i}+$ $m \sum_{j \neq i} p_{j}$ with $a, b, m>0$; this linear direct demand obtains from a representative consumer with the following symmetric and strictly concave quadratic utility function:

$$
U(q)=u_{1} \sum_{i=1}^{n} q_{i}-\frac{1}{2}\left(u_{2} \sum_{i=1}^{n} q_{i}^{2}+2 u_{3} \sum_{j \neq i} q_{i} q_{j}\right),
$$

with $u_{2}>u_{3}>0, u_{1}>0$, and where

$$
\begin{gathered}
a=\frac{u_{1}}{u_{2}+(n-1) u_{3}}, \\
b=\frac{u_{2}+(n-2) u_{3}}{\left[u_{2}+(n-1) u_{3}\right]\left(u_{2}-u_{3}\right)}
\end{gathered}
$$

and

$$
m=\frac{u_{3}}{\left[u_{2}+(n-1) u_{3}\right]\left(u_{2}-u_{3}\right)} .
$$

(See Vives 1999, pp. 146-147.)

The innovation function of firm $i$ is $c_{i}=\bar{c}-x_{i}-\beta \sum_{j \neq i} x_{j}$ and the cost of investing $x$ in R\&D is given by $\Gamma(x)=(\gamma / 2) x^{2}$. Linear demand satisfies Assumption 1B, the innovation and investment functions satisfy Assumptions A.2 and A.3. Under this model specification, we have $v=-b+(n-1) m$, and $v_{\lambda}=-b+\lambda(n-1) m$. According to the above analysis, we impose: $v<0$, i.e. $b>(n-1) m$. 
Simultaneous model. Interior equilibrium. By solving the FOCs and using that in the symmetric equilibrium $q^{*}=a+v p^{*}$, we derive the symmetric interior equilibrium:

$$
p^{*}=\frac{v_{\lambda}(B a \tau-\bar{c} \gamma)+a \gamma}{\Delta}
$$

and

$$
x^{*}=\frac{\tau\left(-v_{\lambda}\right)(\bar{c} v+a)}{\Delta} .
$$

Second-order, stability and regularity conditions. It is straightforward to obtain that

$$
\Delta_{x}=-\gamma, \Delta_{p}=v+v_{\lambda}=-2 b+(n-1) m(1+\lambda), \text { and } \Delta=-\left(v+v_{\lambda}\right) \gamma-v v_{\lambda} B \tau
$$

Because demand is linear, the regularity condition $\Delta_{p}<0$ is implied by the assumption $v<$ 0 . We thus only have to impose the second regularity condition (64), therefore we assume $-\left(v+v_{\lambda}\right) \gamma>v v_{\lambda} B \tau$. Second order conditions are: $\partial_{p_{i} p_{i}} \phi_{i}=-2 b<0, \partial_{x_{i} x_{i}} \phi_{i}=-\gamma<0$ and $\partial_{p_{i} p_{i}} \phi_{i}\left(\partial_{x_{i} x_{i}} \phi_{i}\right)-\left(\partial_{x_{i} p_{i}} \phi_{i}\right)^{2}>0$, which is equivalent to $2 \gamma b>[-b+\lambda(n-1) \beta m]^{2}$.

Table B1: Linear Bertrand Model

\begin{tabular}{l||c}
\hline Demand & $D_{i}(p)=a-b p_{i}+m \sum_{j \neq i} p_{j}$ \\
$c_{i}=$ & $\bar{c}-x_{i}-\beta \sum_{j \neq i} x_{j}$ \\
$\Gamma(x)=$ & $(\gamma / 2) x^{2}$ \\
$v=$ & $-b+(n-1) m$ \\
$v_{\lambda}=$ & $-b+\lambda(n-1) m$ \\
S.O.C & $\gamma b>[-b+\lambda(n-1) \beta m]^{2} / 2$ \\
Regularity Condition & {$\left[-\left(v+v_{\lambda}\right) / v v_{\lambda}\right] \gamma>B \tau$} \\
\hline
\end{tabular}

Comparative statics on $\lambda$ and spillover thresholds. Recall that only $R_{\mathrm{I}}$ exits if $-\Delta_{p}<\left(\partial D_{k}\left(p^{*}\right) / \partial p_{i}\right) \Lambda\left(v / v_{\lambda}\right)$, i.e., if

$$
-\left(v+v_{\lambda}\right)<m \Lambda\left(\frac{v}{v_{\lambda}}\right)
$$

otherwise we can identify $R_{\mathrm{II}}$ and $R_{\mathrm{III}}$ by deriving the corresponding spillover threshold. From (73):

$$
\operatorname{sign}\left\{\frac{\partial x^{*}}{\partial \lambda}\right\}=\operatorname{sign}\left\{\beta\left[-\left(v+v_{\lambda}\right) \frac{v_{\lambda}}{v}-\lambda(n-1) m\right]-m\right\}
$$

Therefore,

$$
\text { if } \beta \leq \underline{\beta}(\lambda) \equiv \frac{m[b-(n-1) m]}{\lambda(n-1)^{2}(\lambda+2) m^{2}-4 b(\lambda+1 / 4)(n-1) m+2 b^{2}},
$$


then $\partial x^{*} / \partial \lambda \leq 0$ and $\partial p^{*} / \partial \lambda>0\left(R_{\mathrm{I}}\right)$. It is easy to see that $\underline{\beta}(\lambda)$ depends only on $m / b$ and that it is hump-shaped in $m / b$ (with $\underline{\beta}(\lambda)=0$ for $m / b=0$ and for $m / b=1 /(n-1)$ ).

Note that $\chi=0, y=1$ and $\xi=\frac{\beta[b-\lambda(n-1) m]^{2}}{m \gamma}$, so

$$
H=\frac{m \gamma}{[b-\lambda(n-1) m]^{2}}
$$

Since $v_{\lambda}<0, H$ is strictly increasing in $\lambda$. Thus,

$$
\operatorname{sign}\left\{\frac{\partial p^{*}}{\partial \lambda}\right\}=\operatorname{sign}\{H-\beta B\} .
$$

It follows that:

$$
\beta^{\prime}=\frac{-1+\sqrt{4 H(n-1)+1}}{2(n-1)}
$$

As $H$ is strictly increasing in $\mathbf{\square}$, so is $\beta^{\prime}$ (in AJ and KMZ $\beta^{\prime}$ is independent of $\lambda$ ).

Figures B1a and B1b depict the spillover thresholds and the three regions. The threshold for $R_{\mathrm{I}}$ and $R_{\mathrm{II}}$ is given by (96), whereas the threshold for $R_{\mathrm{II}}$ and $R_{\mathrm{III}}$ is given by (98). For illustrative purposes we consider two cases that only differ in the number of firms. In Figure B1a, $n=8$, and condition (95) is not satisfied for any value of $\lambda$ if $\beta$ is sufficiently high, and consequently $R_{\mathrm{II}}$ and/or $R_{\mathrm{III}}$ exist. In Figure $\mathrm{B} 1 \mathrm{~b}, n=10$, and condition (95) holds for $\lambda>0.882$. Thus, for $\lambda$ sufficiently high, only $R_{\mathrm{I}}$ exists irrespective of the spillover level.

Spillover thresholds and regions $R_{\mathrm{I}}, R_{\mathrm{II}}$ and $R_{\mathrm{III}}{ }^{8}$

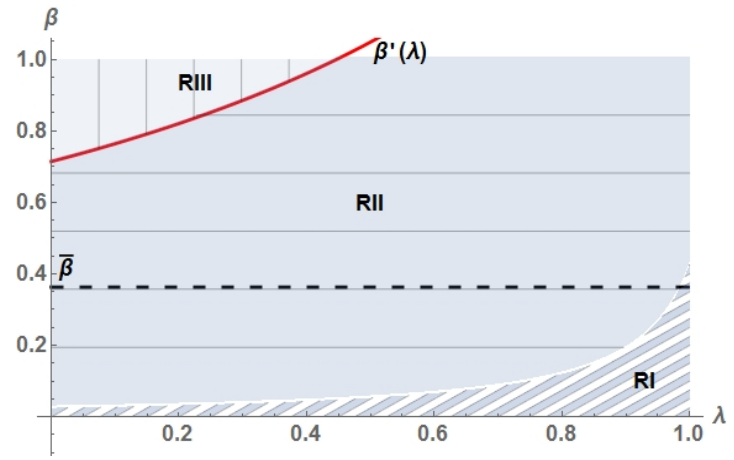

Fig. B1a. Linear Bertrand model.

$$
(n=8)
$$

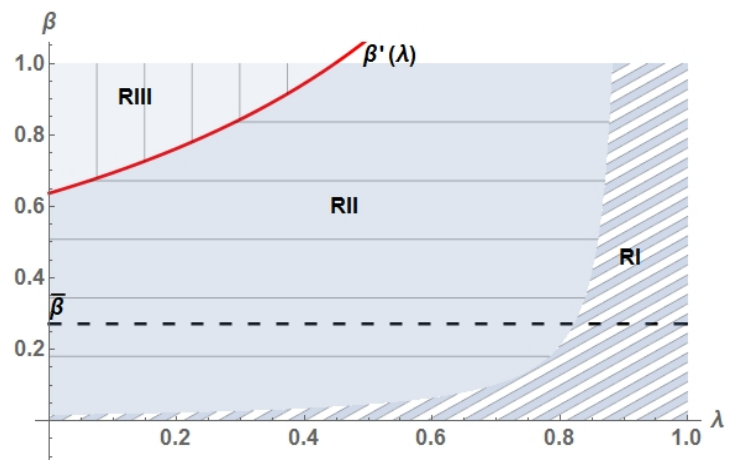

Fig. B1b. Linear Bertrand model.

$$
(n=10)
$$

Comparative statics on $\beta^{\prime}$. Straightforward calculations show that the threshold $\beta^{\prime}(\lambda)$ is strictly decreasing in $b$ and strictly increasing in $\gamma$. These results are in line with the Cournot model. ${ }^{9}$ We also obtain that $\beta^{\prime}(\lambda)$ is strictly increasing in the slope of the direct demand

\footnotetext{
${ }^{8}$ All simulations are conducted for $a=700, \bar{c}=600, b=1.4, m=0.12$ and $\gamma=70$.

${ }^{9}$ In Cournot $\beta^{\prime}(0)$ is strictly increasing in $b$ (see Table A3); recall that $b$ is the (absolute value of the) slope of
} 
with respect to rival prices, $m$. This follows since $H$ has the same properties. However, $H$ is increasing in $u_{3} / u_{2}$ for $u_{3} / u_{2}$ low (local monopolies) and decreasing in $u_{3} / u_{2}$ for $u_{3} / u_{2}$ close to 1 (homogenous products). Therefore $\beta^{\prime}(\lambda)$ is non-monotone in $u_{3} / u_{2}$. It can also be showed that as in AJ and the CE model, $\beta^{\prime}(0)$ is strictly decreasing in $n$ (the threshold does not depend on $n$ in KMZ), and therefore in terms of consumer surplus it is optimal to suppress overlapping ownership for any level of spillovers when firm entry is insufficient. In particular, this is the case in Bertrand with linear demand when $n<m \gamma / b^{2}$, in which case $\beta^{\prime}(0)>1$. More generally, the sign of $\partial \beta^{\prime}(\lambda) / \partial n$ for some $\lambda \in(0,1)$ depends on the level of $\lambda$ and $n$. Numerical simulations show that for low or moderate values of $\lambda, \partial \beta^{\prime}(\lambda) / \partial n<0$, whereas for high $\lambda, \partial \beta^{\prime}(\lambda) / \partial n>0$ if $n$ is sufficiently high. ${ }^{10}$

PROPOSITION BL1 Under the linear demand specification, if $-\left(v+v_{\lambda}\right)<m \Lambda\left(v / v_{\lambda}\right)$ then only region $R_{\mathrm{I}}$ exists. Otherwise, assume $n>H(1)$, where $H$ is given by (97), and let $\underline{\beta}(\lambda)$ and $\beta^{\prime}$ be given, respectively, by (96) and (98). Then the following statements hold:

(i) if $\beta \leq \underline{\beta}(\lambda)$, then $\frac{\partial q^{*}}{\partial \lambda}<0$ and $\frac{\partial x^{*}}{\partial \lambda} \leq 0\left(R_{\mathrm{I}}\right)$;

(ii) if $\underline{\beta}(\lambda)<\beta \leq \beta^{\prime}(\lambda)$, then $\frac{\partial q^{*}}{\partial \lambda} \leq 0$ and $\frac{\partial x^{*}}{\partial \lambda}>0\left(R_{\mathrm{II}}\right)$;

(iii) if $\beta>\beta^{\prime}(\lambda)$, then $\frac{\partial q^{*}}{\partial \lambda}>0$ and $\frac{\partial x^{*}}{\partial \lambda}>0\left(R_{\mathrm{III}}\right)$.

We have that both $\underline{\beta}(\lambda)$ and $\beta^{\prime}(\lambda)$ are increasing in $\lambda$ and hump-shaped in $u_{3} / u_{2}$, and $\partial \beta^{\prime}(0) / \partial n<$ 0 .

Profit. Simulations show that also in Bertrand with differentiated products and linear demand, profit in equilibrium is strictly increasing in the degree of overlapping ownership: $\pi^{* \prime}(\lambda)>0$.

Welfare. First, we derive the threshold, $\bar{\beta}$, above which welfare increases with $\lambda$ starting from $\lambda=0$. We obtain $\bar{\beta}$ from the condition $W^{\prime}(0)>0$. Using (80), we only have to solve $\left.\digamma\right|_{\lambda=0}=0$ for $\beta$ to obtain the expression for $\bar{\beta}$. In particular, we have to solve

$$
-\frac{\beta B v}{m}+\frac{v}{v_{\lambda}^{2}} \gamma-\beta(n-1)\left[\frac{\beta(-b+v)}{m}+\frac{v}{v_{\lambda}}\right]=0
$$

or, equivalently,

$$
v_{\lambda}^{2}(n-1)(b-2 v) \beta^{2}-v_{\lambda} v\left[v_{\lambda}+m(n-1)\right] \beta+v \gamma m=0 .
$$

the inverse demand in Cournot, while it is the slope of the direct demand with respect to own price in Bertrand.

${ }^{10}$ For example, for $b=1.5, m=0.1$ and $\gamma=60, \partial \beta^{\prime}(\lambda) / \partial n<0$ for $n=2.5$, but $\partial \beta^{\prime}(\lambda) / \partial n>0$ for $n=6$ and $\lambda>0.87$. 
The above equation has two roots, only one of them can be positive since the denominator of the roots is $-2 b(n-1)(b-2 v)<0$. Thus, $\bar{\beta}$ is given by

$$
\bar{\beta}=\frac{v^{2}-\sqrt{v\left\{v(n-1)^{2} m^{2}+2[(4 \gamma-b) v-2 b \gamma](n-1) m+b^{2} v\right\}}}{-2 b(n-1)(b-2 v)} .
$$

Numerical simulations confirm that the spillover thresholds satisfy $\beta^{\prime}(0)>\bar{\beta}$.

Table B2: $H$ and Spillover Thresholds in Linear Bertrand Model

\begin{tabular}{l|c}
\hline$H=$ & $m \gamma /[b-\lambda(n-1) m]^{2}$ \\
$\underline{\beta}(\lambda)=$ & $m[b-(n-1) m] /\left[\lambda(n-1)^{2}(\lambda+2) m^{2}-4 b(\lambda+1 / 4)(n-1) m+2 b^{2}\right]$ \\
$\overline{\bar{\beta}}=$ & $\left(v^{2}-\sqrt{v\left\{v(n-1)^{2} m^{2}+2[(4 \gamma-b) v-2 b \gamma](n-1) m+b^{2} v\right\}}\right) /[-2 b(n-1)(b-2 v)]$ \\
$\beta^{\prime}=$ & $(-1+\sqrt{4 H(n-1)+1}) /[2(n-1)]$ \\
\hline
\end{tabular}

Comparative statics on $\bar{\beta}$. As in Cournot (in AJ, KMZ and CE), the threshold $\bar{\beta}$ decreases with $n$. Similarly and in line with Cournot: $\bar{\beta}$ decreases with the slope of demand and increases with the parameter of the slope for the investment cost, $\gamma$. Regarding product differentiation: $\bar{\beta}$ is hump-shaped in $u_{3} / u_{2}$ since $\bar{\beta}=0$ both for $u_{3} / u_{2}=0$ and $u_{3} / u_{2}=1$. Finally, also in Bertrand $\bar{\beta}$ may take values greater than 1 (so $\lambda_{\mathrm{TS}}^{o}=0$ irrespective of the value of $\beta$ ) when there are a few firms in the market and $\gamma(b)$ are sufficiently high (low). Note that in Figures B2a-c we assume that parameters $a, b$ and $m$ are fixed as $n$ changes. This implies that parameters $u_{1}, u_{2}$ and $u_{3}$ must change with $n$ (see Section B.4.1). Alternatively, we assume in Figure B2d that parameters $u_{1}, u_{2}$ and $u_{3}$ are fixed (such that $a=750, b=1.5$ and $m=0.1$ for $n=8$ ), while $a, b$ and $m$ change with $n$. Results are qualitatively the same: the thresholds in B2a and B2d are almost the same for $n$ equal or close to 8, while they are higher in B2d than in B2a for two or three firms in the market. 
Threshold value $\bar{\beta}$

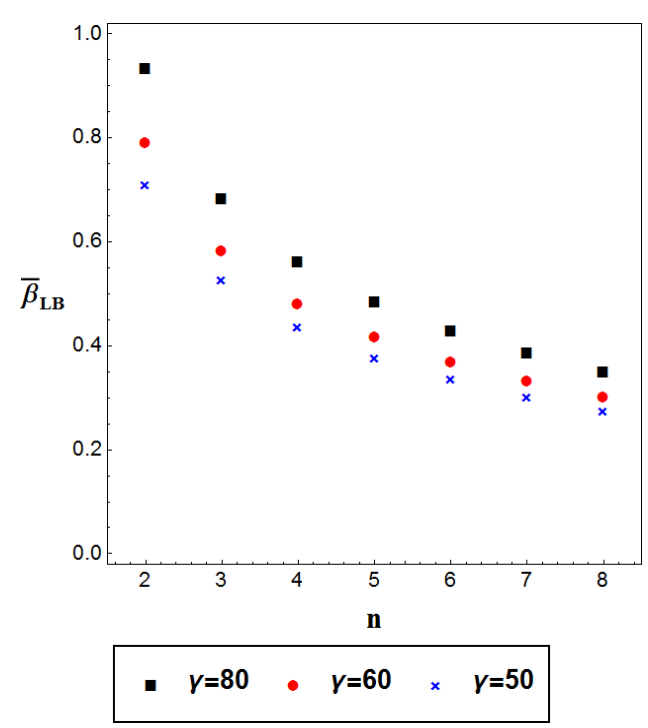

Fig. B2a. Linear Bertrand model.

$$
(b=1.5, m=0.1)
$$

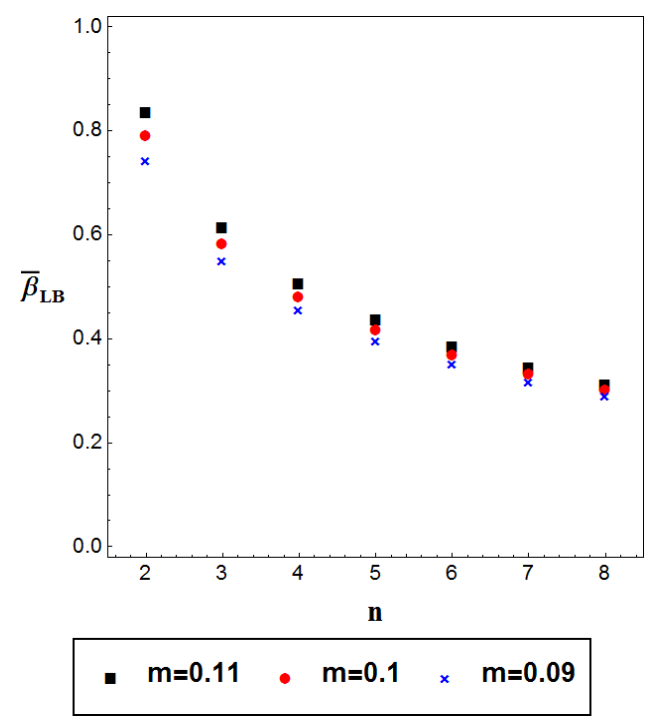

Fig. B2c. Linear Bertrand model.

$$
(b=1.5, \gamma=60)
$$

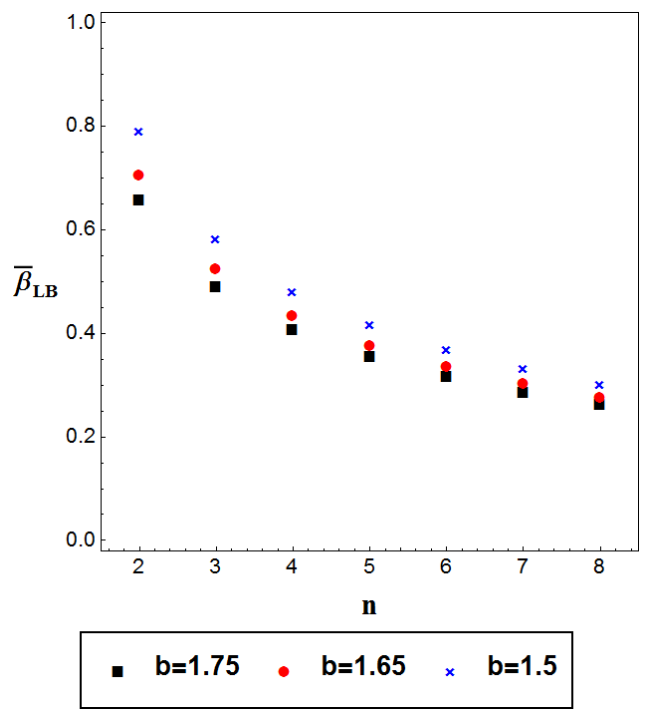

Fig. B2b. Linear Bertrand model.

$$
(\gamma=60, m=0.1)
$$

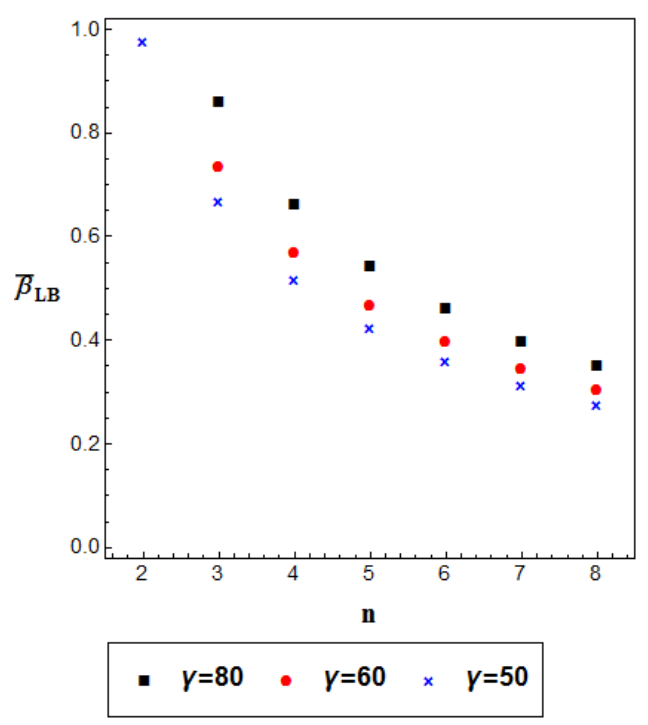

Fig. B2d. Linear Bertrand model. $\left(u_{1}=937.5, u_{2}=0.7\right.$ and $\left.u_{3}=0.078\right)$ 
Optimal degree of overlapping ownership (TS and CS standard $)^{11}$

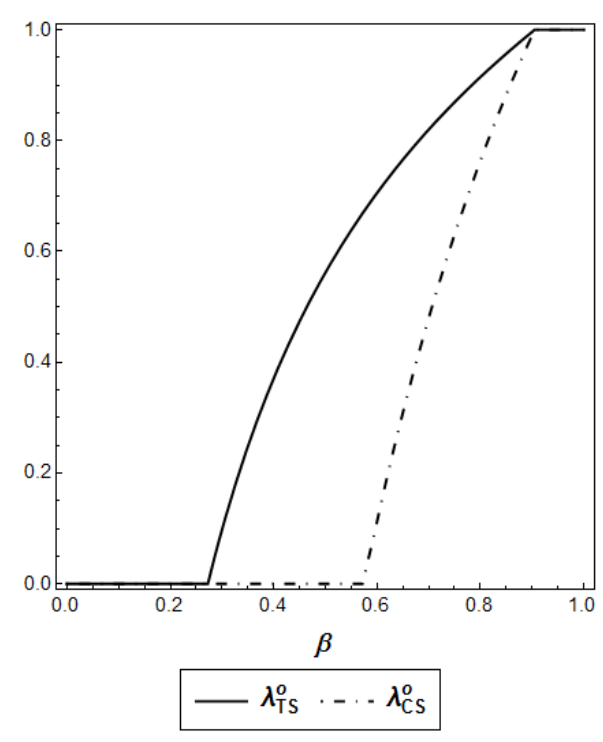

Fig. B3a. Linear Bertrand model.

$$
(\gamma=50, n=6)
$$

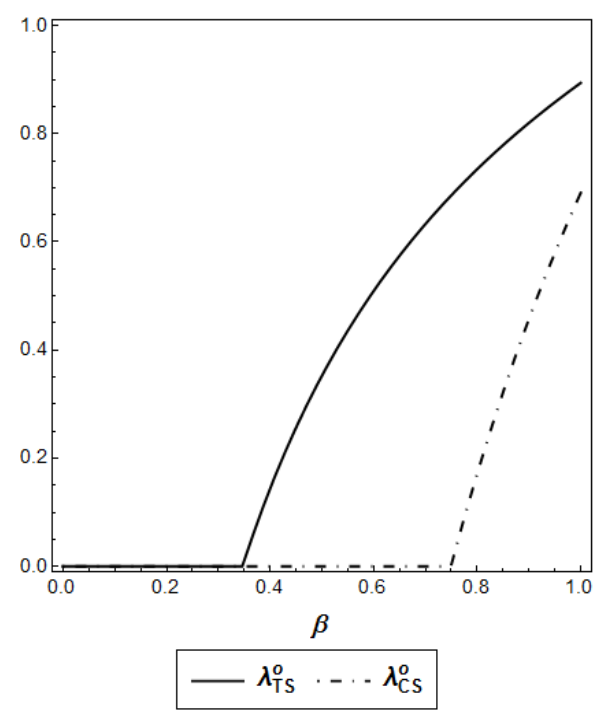

Fig. B3c. Linear Bertrand model.

$$
(\gamma=80, n=6)
$$

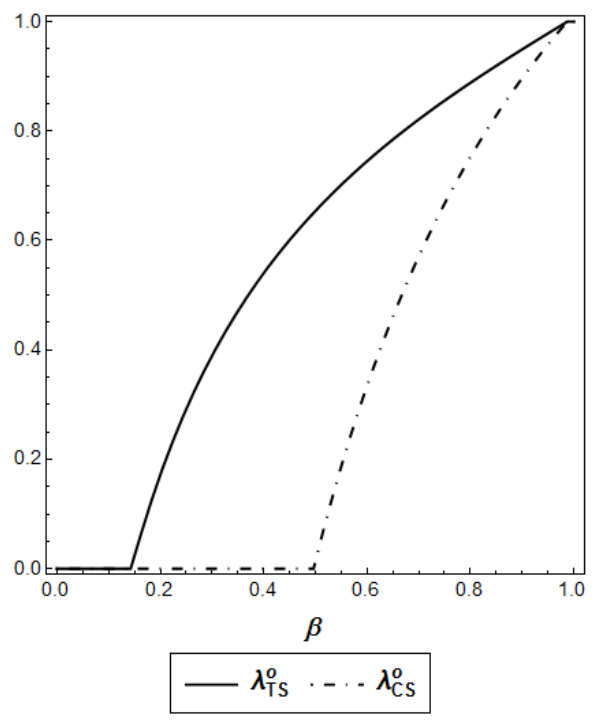

Fig. B3b. Linear Bertrand model.

$$
(\gamma=50, n=8)
$$

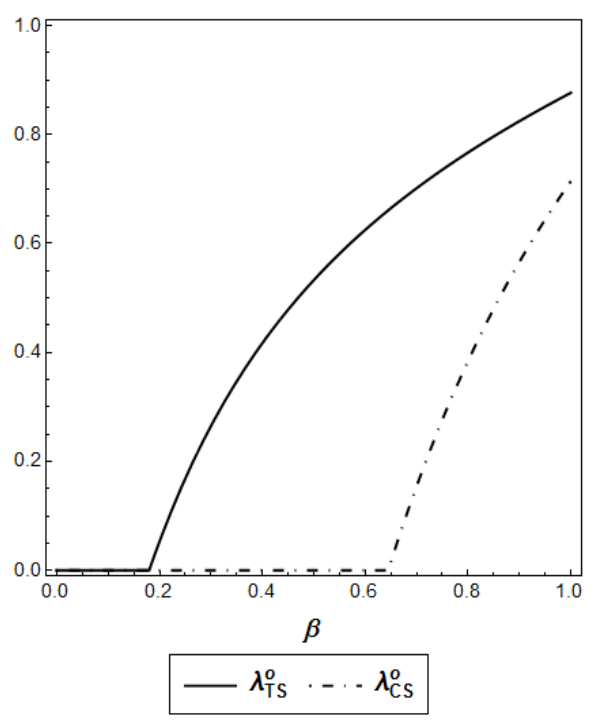

Fig. B3d. Linear Bertrand model.

$$
(\gamma=80, n=8)
$$

Comparative statics on the socially optimal degree of overlapping ownership. Our simulations confirm that the main findings obtained in Cournot also hold in Bertrand; namely the socially optimal level of overlapping ownership increases with the size of spillovers and with the number of firms. Secondly, while the comparative statics are qualitatively similar in terms of consumer surplus, the scope for overlapping ownership is lower. Thirdly, Figures B3a-d show that for not

\footnotetext{
${ }^{11}$ All simulations are conducted for $a=700, b=1.5, m=0.1$ and $\bar{c}=500$.
} 
too highly concentrated markets and high spillover levels, $\lambda=1$ can be optimal in terms of total and consumer surplus. The thresholds $\bar{\beta}$ and $\beta^{\prime}(0)$, as discussed above, decrease with $n$, and the optimal degrees of overlapping ownership $\lambda_{\mathrm{TS}}^{o}$ and $\lambda_{\mathrm{CS}}^{o}$, decrease with the parameter of the slope for the investment cost, $\gamma$.

Optimal degree of overlapping ownership (TS and CS standard) ${ }^{12}$

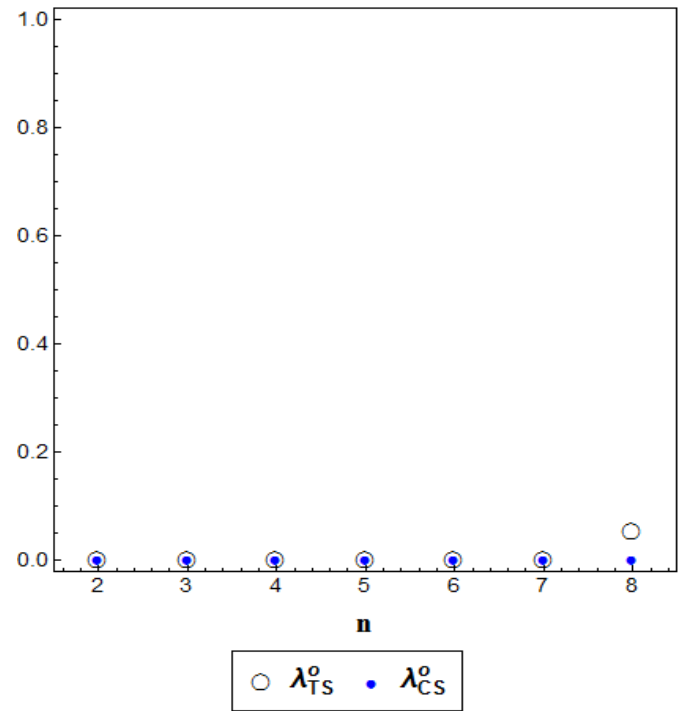

Fig. B4a. Linear Bertrand model.

$$
(\gamma=80, \beta=0.2)
$$

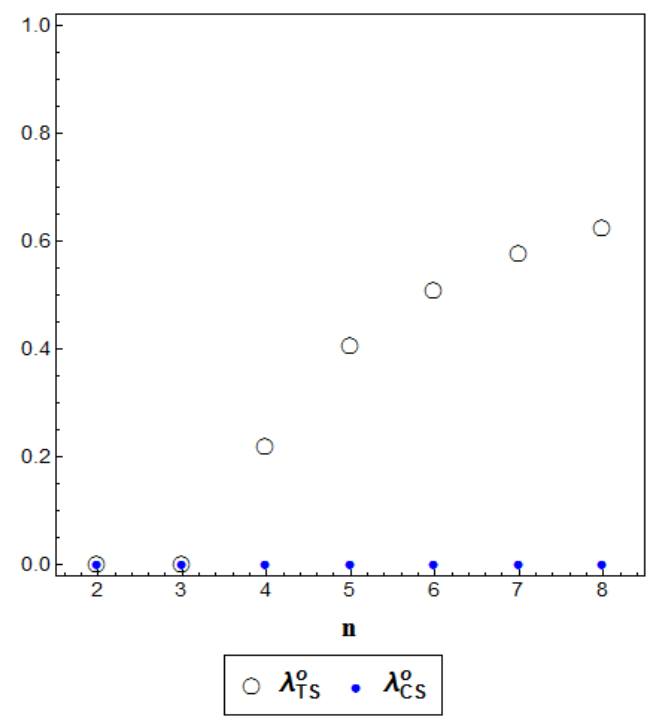

Fig. B4c. Linear Bertrand model.

$$
(\gamma=80, \beta=0.6)
$$

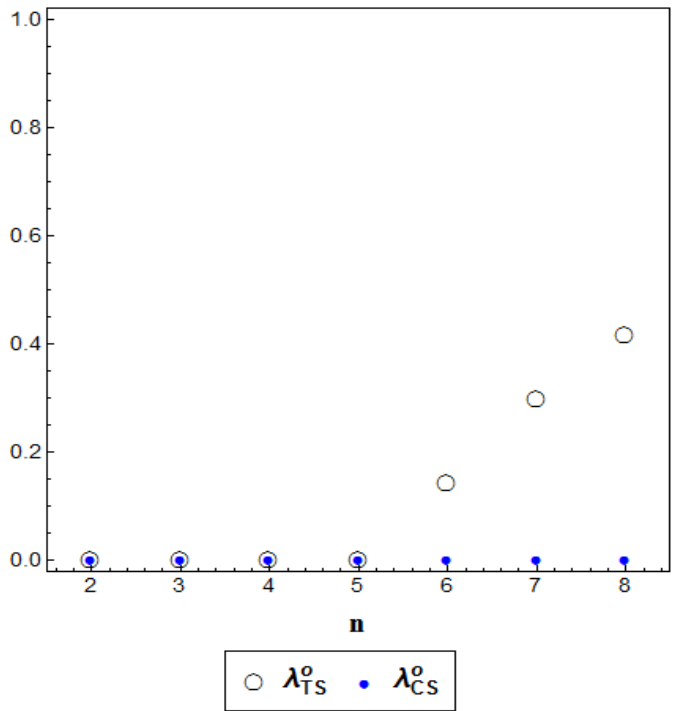

Fig. B4b. Linear Bertrand model.

$$
(\gamma=80, \beta=0.4)
$$

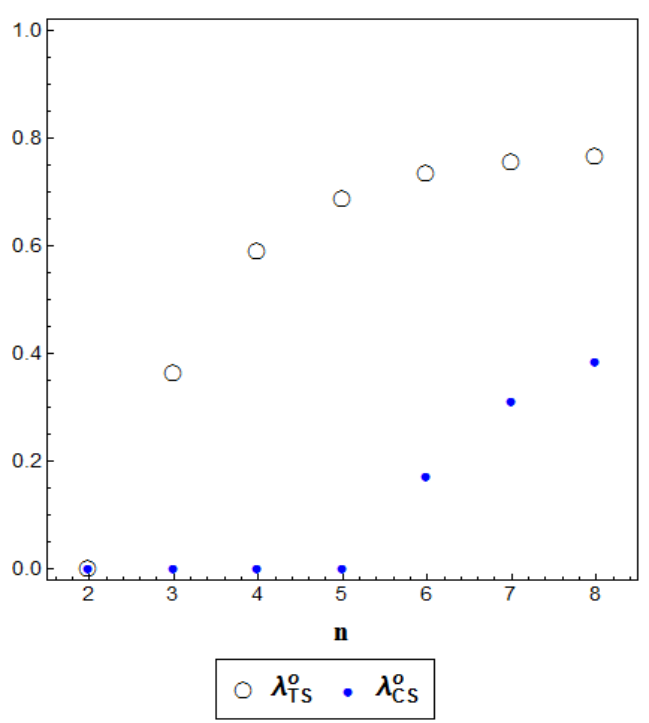

Fig. B4d. Linear Bertrand model.

$$
(\gamma=80, \beta=0.8)
$$

\footnotetext{
${ }^{12}$ All simulations are conducted for $a=750, b=1.5, m=0.1$ and $\bar{c}=500$.
} 


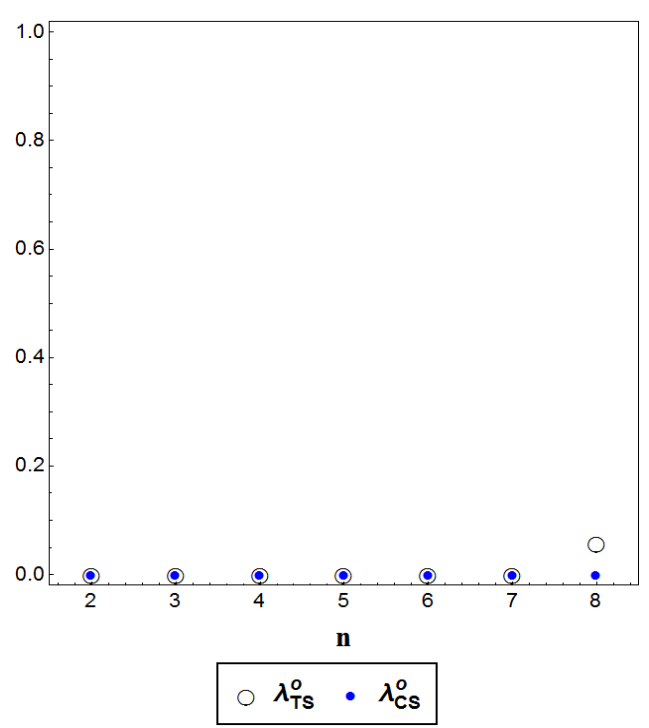

Fig. B4e. Linear Bertrand model.

$$
\begin{gathered}
\left(\gamma=80, \beta=0.2, u_{1}=937.5, u_{2}=0.7\right. \\
\text { and } \left.u_{3}=0.078\right)
\end{gathered}
$$

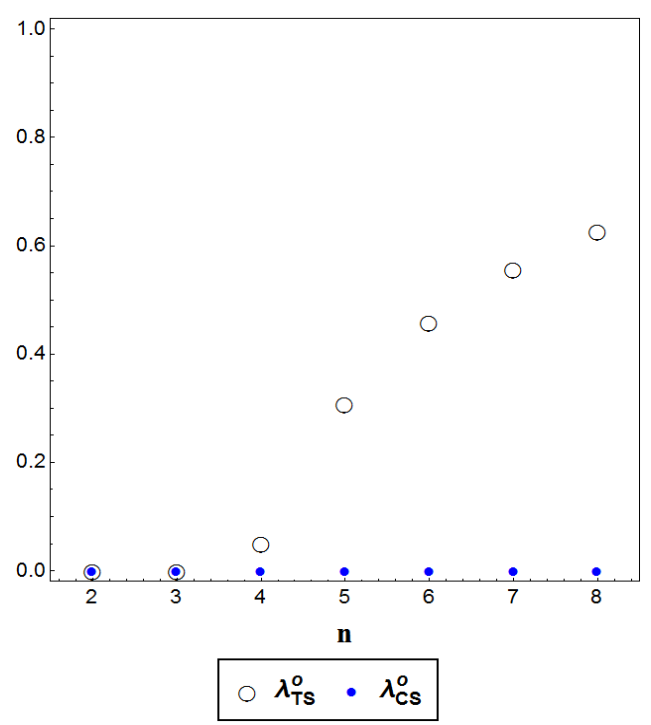

Fig. B4g. Linear Bertrand model.

$$
\begin{gathered}
\left(\gamma=80, \beta=0.6, u_{1}=937.5, u_{2}=0.7\right. \\
\text { and } \left.u_{3}=0.078\right)
\end{gathered}
$$

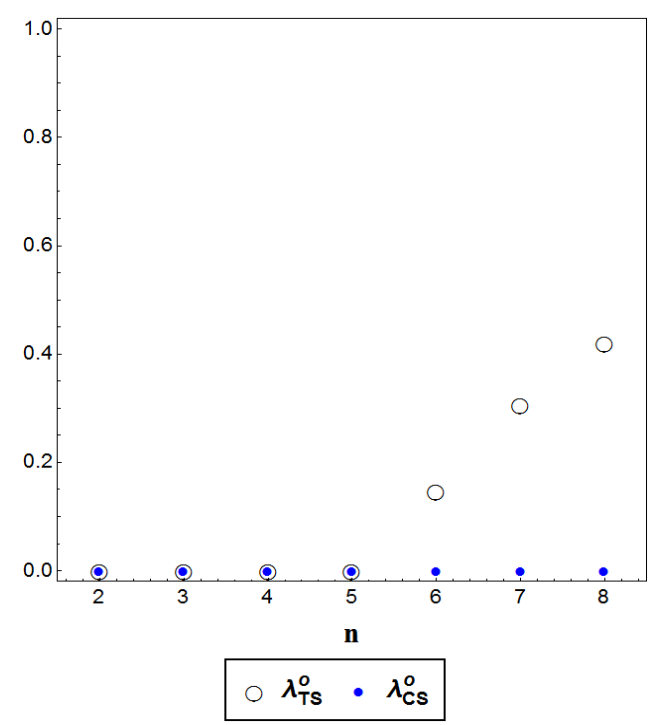

Fig. B4f. Linear Bertrand model.

$$
\begin{gathered}
\left(\gamma=80, \beta=0.4, u_{1}=937.5, u_{2}=0.7\right. \\
\text { and } \left.u_{3}=0.078\right)
\end{gathered}
$$

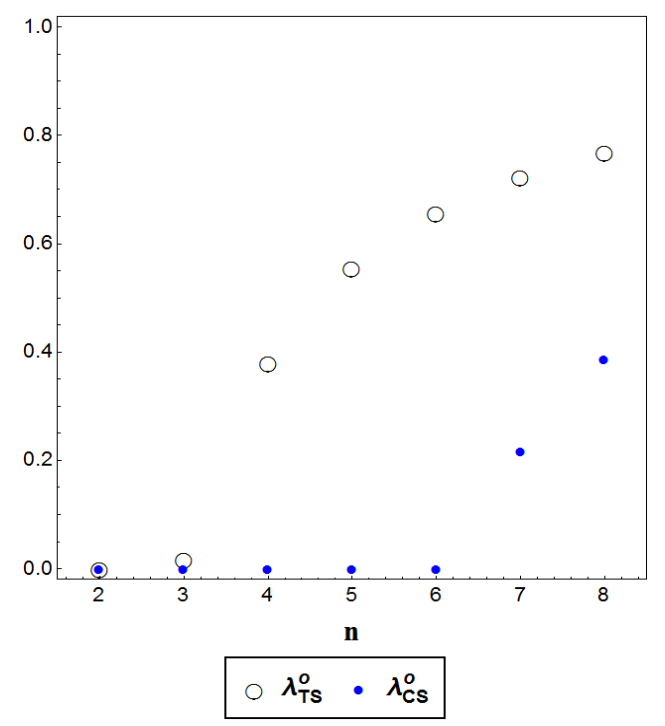

Fig. B4h. Linear Bertrand model.

$$
\begin{gathered}
\left(\gamma=80, \beta=0.8, u_{1}=937.5, u_{2}=0.7\right. \\
\text { and } \left.u_{3}=0.078\right)
\end{gathered}
$$

Finally, as Figures B4a-d indicate, it is not optimal to allow overlapping ownership for highly concentrated markets. As in the case of output competition, $\lambda_{\text {TS }}^{o}$ increases weakly with the number of firms, and as in $\mathrm{AJ}$ and $\mathrm{CE}, \lambda_{\mathrm{CS}}^{o}$ increases weakly with the number of firms and only if $n$ is sufficiently large given the size of the spillover. ${ }^{13}$ In Figures B4a-d we keep

\footnotetext{
${ }^{13}$ Recall that in KMZ the threshold $\beta^{\prime}$, and therefore $\operatorname{sign}\left\{C S^{\prime}(\lambda)\right\}$, are independent of the number of firms.
} 
parameters $a, b$ and $m$ fixed as $n$ changes, so parameters $u_{1}, u_{2}$ and $u_{3}$ must change with $n$. In Figures B4e-h, however, we allow parameters $a, b$ and $m$ to change with $n$ by setting $u_{1}, u_{2}$ and $u_{3}$ at values such that $a=750, b=1.5$ and $m=0.1$ for $n=8$. Results are qualitatively the same in the two cases.

Optimal degree of overlapping ownership (TS and CS standard) $)^{14}$

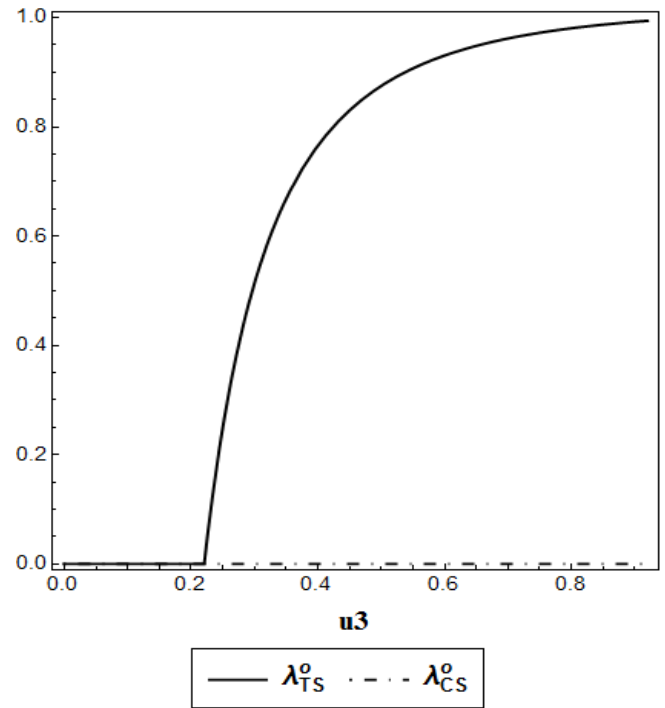

Fig. B5a. Linear Bertrand model.

$$
(\beta=0, \gamma=150, n=5)
$$

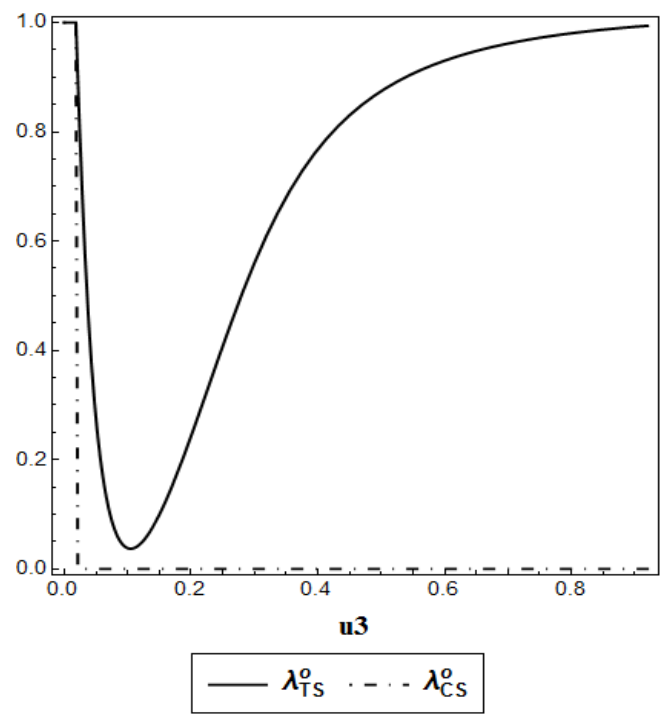

Fig. B5c. Linear Bertrand model.

$$
(\beta=0.75, \gamma=150, n=5)
$$

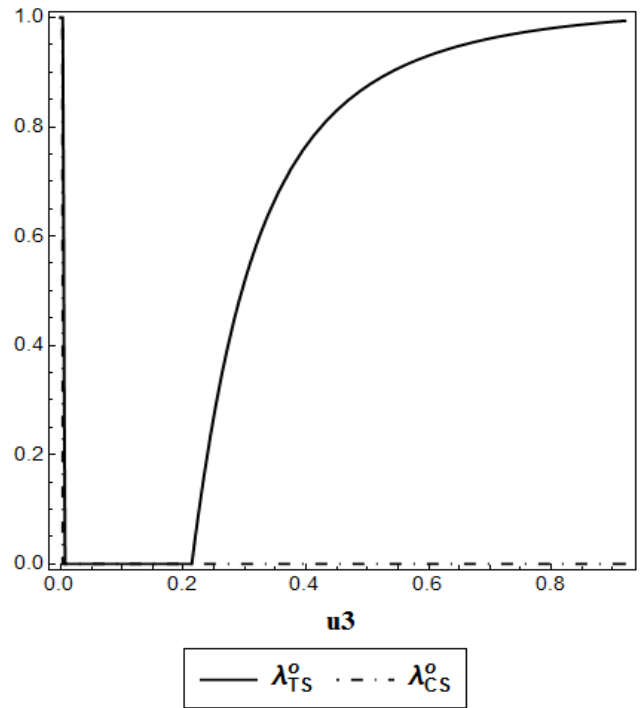

Fig. B5b. Linear Bertrand model.

$$
(\beta=0.25, \gamma=150, n=5)
$$

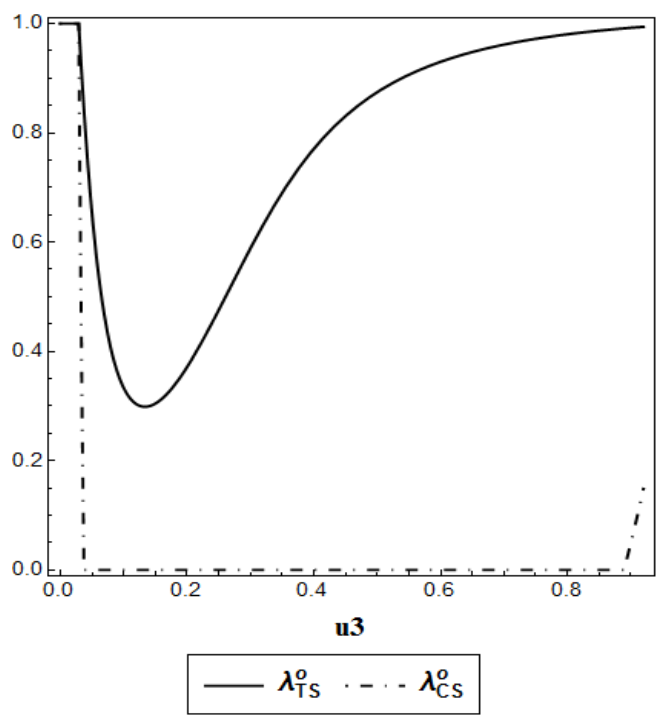

Fig. B5d. Linear Bertrand model.

$$
(\beta=1, \gamma=150, n=5)
$$

Comparative statics on the degree of product differentiation. Here, we fix $u_{2}=1$, and we then compute the optimal degrees of overlapping ownership ( $\lambda_{\mathrm{TS}}^{o}$ and $\lambda_{\mathrm{CS}}^{o}$ ) for values of $u_{3}$

\footnotetext{
${ }^{14}$ All simulations are conducted for $a=700$ and $\bar{c}=500$.
} 
ranging from 0 (which reflects the monopoly case) to 0.92 (which reflects the case of intense competition because of very low product differentiation). To guarantee that the regularity condition is satisfied for $u_{3} \in[0,0.92]$ we consider $n=5$ and $\gamma=150$. Simulations show that for $\beta>0, \lambda_{\mathrm{TS}}^{o}$ is $\mathrm{U}$-shaped, and so is $\lambda_{\mathrm{CS}}^{o}$ is for $\beta$ sufficiently high (see Figures B5a-d). For $\beta>0$, if $u_{3} \rightarrow 0$, then $\lambda_{\mathrm{TS}}^{o}, \lambda_{\mathrm{CS}}^{o} \rightarrow 1$. The U-shaped pattern is robust and also appears for higher/lower values of $n$ and $\gamma$. In particular, in Figures B6a-b we conduct similar simulations but assuming $n=8$ and $\gamma=60$.

Optimal degree of overlapping ownership (TS and CS standard) $)^{15}$

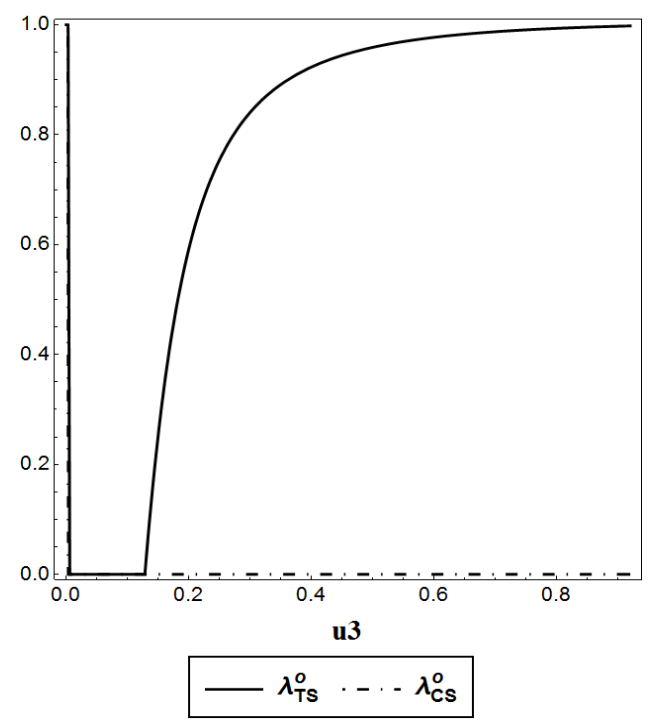

Fig. B6a. Linear Bertrand model. $(\beta=0.1, \gamma=60, n=8)$

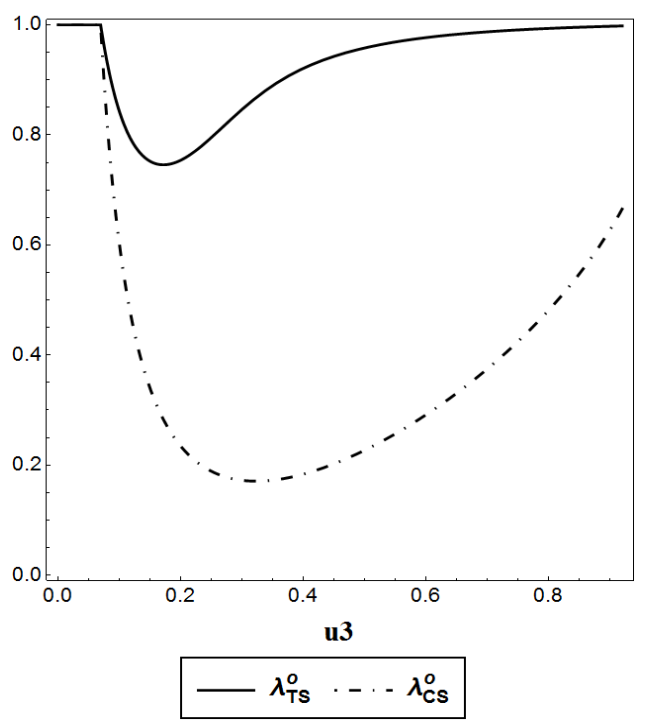

Fig. B6b. Linear Bertrand model. $(\beta=0.9, \gamma=60, n=8)$

Two-stage model. Interior equilibrium. By solving the FOCs (87) and (88) with $c=$ $\bar{c}-B x^{*}$ and $q^{*}=a+v p^{*}$, we obtain the symmetric interior equilibrium:

$$
p^{*}=\frac{\{\bar{c} \gamma-a[(n-1) s(\lambda)+\tau] B\} v_{\lambda}-a \gamma}{\{\gamma+v[(n-1) s(\lambda)+\tau] B\} v_{\lambda}+v \gamma}
$$

and

$$
x^{*}=\frac{v_{\lambda}(\bar{c} v+a)[(n-1) s(\lambda)+\tau]}{\{\gamma+v[(n-1) s(\lambda)+\tau)] B\} v_{\lambda}+v \gamma},
$$

where $s(\lambda) \equiv \omega(\lambda)(\tilde{\beta}(\lambda)-\beta)$, and $\omega(\lambda)$ and $\tilde{\beta}(\lambda)$ are obtained below.

Strategic effect. Here, we first obtain $\partial p_{j}^{*}(\mathbf{x}) / \partial x_{i}$, and we then derive the expressions for the strategic effect of investment $(\psi)$ and the threshold $\tilde{\beta}(\lambda)$. With linear demand we have $\partial_{x_{i} p_{i}} \phi_{i}(\mathbf{x})=-c^{\prime}(B x)[-b+\lambda(n-1) \beta m]$ and $\partial_{x_{j} p_{i}} \phi_{i}(\mathbf{x})=-c^{\prime}(B x)[-b \beta+\lambda m+\lambda(n-2) \beta m]$.

\footnotetext{
${ }^{15}$ All simulations are conducted for $a=700$ and $\bar{c}=500$.
} 
We also have that $\partial_{p_{i} p_{i}} \phi_{i}(\mathbf{x})=-2 b$ and $\partial_{p_{i} p_{j}} \phi_{i}(\mathbf{x})=m(1+\lambda)$. Therefore,

$$
\begin{gathered}
\partial_{p_{i} p_{i}} \phi_{i}(\mathbf{x})-\partial_{p_{i} p_{j}} \phi_{i}(\mathbf{x})=-2 b-m(1+\lambda), \\
\partial_{p_{i} p_{i}} \phi_{i}(\mathbf{x})+(n-1) \partial_{p_{i} p_{j}} \phi_{i}(\mathbf{x})=-2 b+(n-1) m(1+\lambda), \\
\partial_{p_{i} p_{j}} \phi_{i}(\mathbf{x})-\partial_{p_{i} p_{i}} \phi_{i}(\mathbf{x}) \beta=m(1+\lambda)+2 b \beta .
\end{gathered}
$$

Using (36) we can write

$$
\frac{\partial}{\partial x_{i}} p_{j}^{*}(\mathbf{x})=\frac{-c^{\prime}(B x)}{\Omega}[\varphi(\lambda) \beta-(1-\lambda) b m]
$$

where $\Omega=[-2 b-m(1+\lambda)][-2 b+(n-1) m(1+\lambda)]>0$, and $\varphi(\lambda)=\lambda(1+\lambda)(n-1) m^{2}+$ $2 \lambda(n-2) b m-2 b^{2}<0$, since $\varphi(0)=-2 b^{2}<0, \varphi(1)=2(b+m)[-b+m(n-1)]<0$ and $\varphi^{\prime}(\lambda)>0$. Therefore,

$$
\frac{\partial}{\partial x_{i}} p_{j}^{*}(\mathbf{x})<0
$$

From (91) we may write

$$
\begin{aligned}
\frac{\partial}{\partial p_{j}} \phi_{i}\left(\mathbf{p}^{*}(\mathbf{x}), \mathbf{x}, \lambda\right) & =\frac{-q^{*}}{v_{\lambda}}\{m[1+\lambda(n-2)]-b \lambda\}+\lambda q^{*} \\
& =-\frac{q^{*}}{v_{\lambda}} m(1-\lambda) \Lambda .
\end{aligned}
$$

Note that $(1-\lambda) \Lambda$ is strictly positive for all $\lambda<1$, thus, and as expected, for $\lambda<1$ :

$$
\frac{\partial}{\partial p_{j}} \phi_{i}\left(\mathbf{p}^{*}(\mathbf{x}), \mathbf{x}, \lambda\right)>0
$$

Therefore, the strategic effect of investment is

$$
\begin{aligned}
\psi & \equiv(n-1) \frac{\partial \phi_{i}}{\partial p_{j}}\left(\frac{\partial p_{j}^{*}}{\partial x_{i}}\right) \\
& =-(n-1) \frac{q^{*}}{v_{\lambda}} m(1-\lambda) \Lambda\left(\frac{-c^{\prime}(B x)}{\Omega}[\varphi(\lambda) \beta-(1-\lambda) b m]\right) \\
& =\frac{-c^{\prime}(B x)}{\Omega}\left(-\frac{q^{*}}{v_{\lambda}}\right) m(n-1)(1-\lambda) \Lambda[\varphi(\lambda) \beta-(1-\lambda) b m]<0 .
\end{aligned}
$$

We can rewrite the strategic effect of investment as

$$
\psi=-c^{\prime}(B x) q^{*} \omega(\lambda)(\tilde{\beta}(\lambda)-\beta)
$$


where

$$
\omega(\lambda)=\frac{m(n-1)(1-\lambda) \Lambda \varphi(\lambda)}{\Omega v_{\lambda}}>0 \text { and } \tilde{\beta}(\lambda)=\frac{1-\lambda}{\varphi(\lambda)} b m<0 .
$$

Welfare. The expression for $W^{\prime}(\lambda)$ is given by (94). Recall that in Cournot only when the strategic effect is negative, the sign of the impact of $\lambda$ on welfare in each region $\left(R_{\mathrm{I}}, R_{\mathrm{II}}\right.$ and $\left.R_{\mathrm{III}}\right)$ is the same in the simultaneous and the two-stage model. The reason is that the factor that multiplies $\partial x^{*} / \partial \lambda$ in the expression for $W^{\prime}(\lambda)$ is positive. When the strategic effect is positive and spillovers are low, the factor is negative and as a result, welfare decreases with $\lambda$ in $R_{\mathrm{II}}$, and can increase or decrease with $\lambda$ in $R_{\mathrm{I}}$ and in $R_{\mathrm{III}}$. In the Bertrand model with linear demand, the strategic effect is always negative, and as in Cournot, the factor that multiplies $\partial x^{*} / \partial \lambda$ is positive. (Note also that $-1 / v_{\lambda}>0$.) Therefore, the sign of the impact of $\lambda$ on welfare in each region $\left(R_{\mathrm{I}}, R_{\mathrm{II}}\right.$ and $\left.R_{\mathrm{III}}\right)$ is the same in the simultaneous and the two-stage model: $W^{\prime}(\lambda)<0$ when $x^{*}$ decreases and $p^{*}$ increases with $\lambda$ (as in $R_{\mathrm{I}}$ ), $W^{\prime}(\lambda)>0$ when $x^{*}$ increases and $p^{*}$ decreases with $\lambda$ (as in $R_{\mathrm{III}}$ ), and $W^{\prime}(\lambda) \gtrless 0$ when $x^{*}$ and $p^{*}$ increase with $\lambda$ (as in $R_{\mathrm{II}}$ ).

The next figures depict the threshold $\bar{\beta}_{L B}^{2 S}$ above which welfare increases with $\lambda$ at $\lambda=0$. 
Threshold value $\bar{\beta}^{16}$

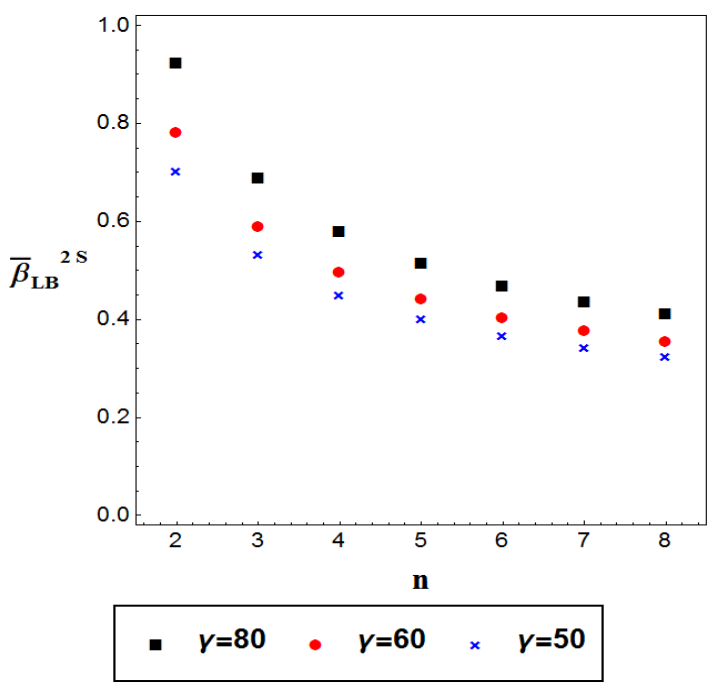

Fig. B7a. Linear Bertrand two-stage model. $(b=1.5, m=0.1)$

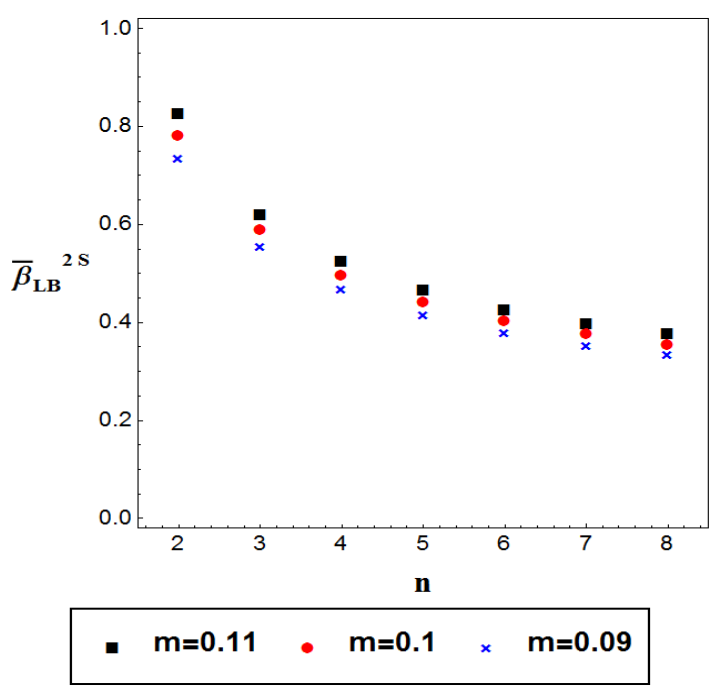

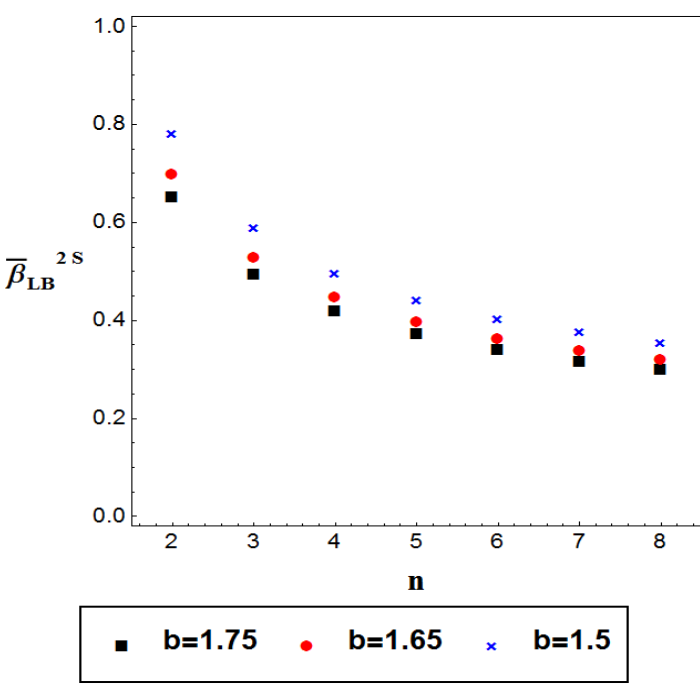

Fig. B7b. Linear Bertrand two-stage model. $(m=0.1, \gamma=60)$

Fig. B7c. Linear Bertrand two-stage model.

$$
(b=1.5, \gamma=60)
$$

Comparative statics on $\bar{\beta}_{L B}^{2 S}$. Results are consistent with those obtained in Cournot and in simultaneous Bertrand: the threshold $\bar{\beta}_{L B}^{2 S}$ increases with $m$ and $\gamma$, and decreases with $n$ and with $b$. In addition, and in line with the other models, $\bar{\beta}_{L B}^{2 S}$ may be greater than 1 (and thus $\lambda_{\mathrm{TS}}^{o}=0$ for all $\beta$ ) when there are few firms in the market and $\gamma(b)$ are sufficiently high (low).

\footnotetext{
${ }^{16}$ In the three simulations: $a=900$ and $\bar{c}=500$.
} 
Optimal degree of overlapping ownership (TS and CS standard) $)^{17}$
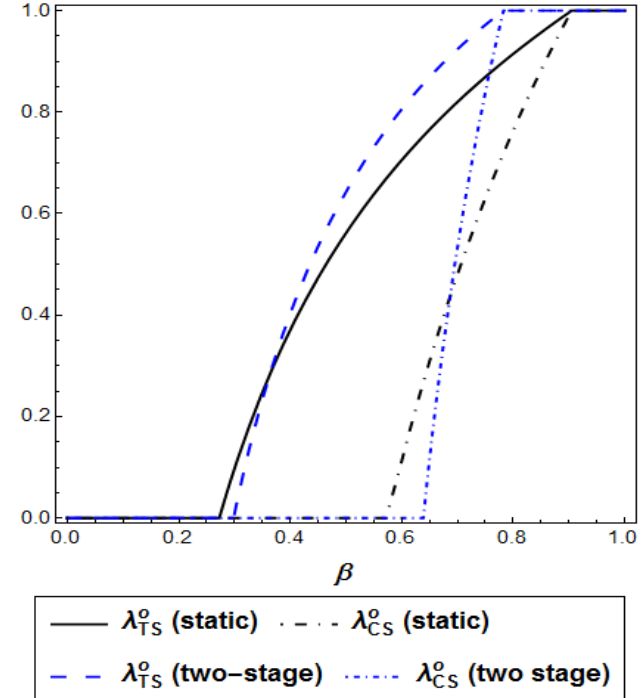

Fig. B8a. Linear Bertrand two-stage model. $(\gamma=50, n=6)$
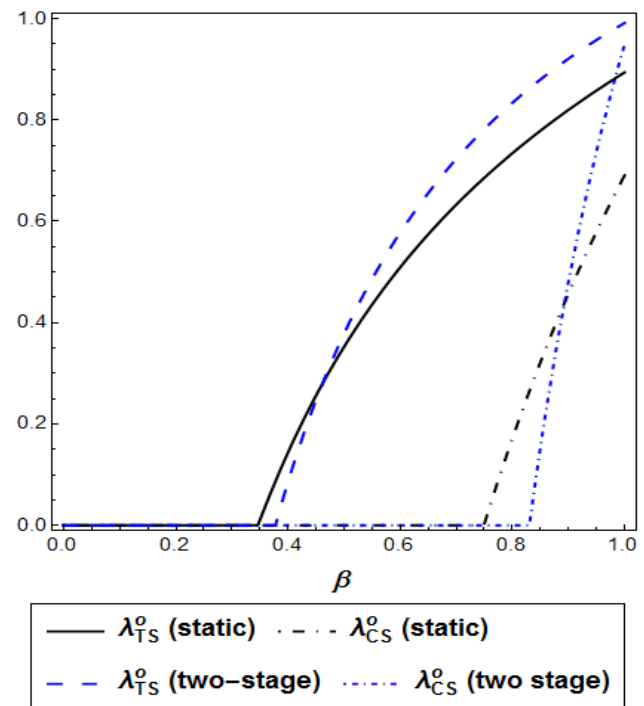

Fig. B8c. Linear Bertrand two-stage model. $(\gamma=80, n=6)$

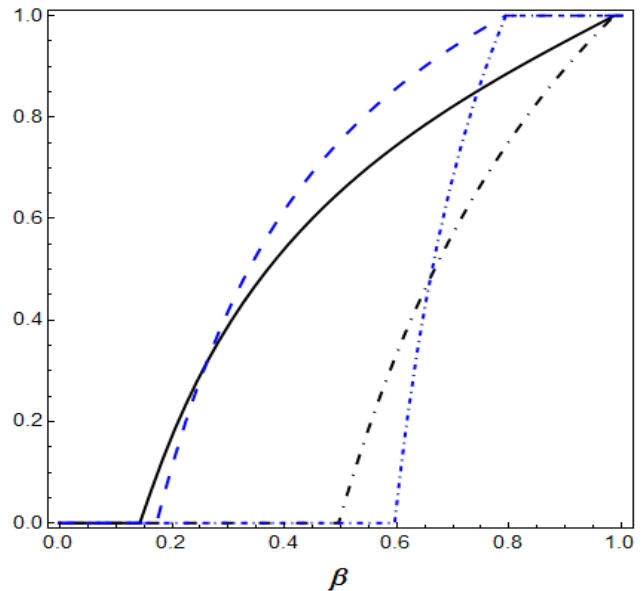

$-\lambda_{\mathrm{TS}}^{o}$ (static) $\ldots \lambda_{\mathrm{CS}}^{o}$ (static)

- $\lambda_{\mathrm{TS}}^{o}$ (two-stage) $\ldots . . . \lambda_{\mathrm{CS}}^{o}$ (two stage)

Fig. B8b. Linear Bertrand two-stage model. $(\gamma=50, n=8)$

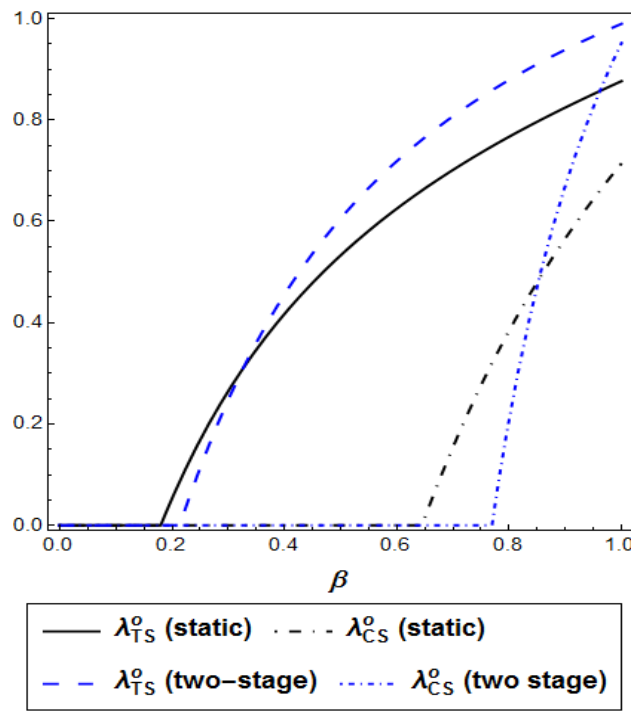

Fig. B8d. Linear Bertrand two-stage model. $(\gamma=80, n=8)$

Comparative statics on the socially optimal degree of overlapping ownership. Results are similar to those obtained in Cournot with two stages: $\lambda_{\text {TS }}^{o}$ increases with $\beta$ and $n$, and when $\mathrm{R} \& \mathrm{D}$ has commitment value $\lambda_{\mathrm{TS}}^{o}$ tends to be higher than in the simultaneous model when spillovers are high. However and unlike the Cournot model, we do not observe cases in which $\lambda_{\mathrm{CS}}^{o}>\lambda_{\mathrm{TS}}^{o}$. The reason is that those cases may arise in Cournot when the strategic effect is

\footnotetext{
${ }^{17}$ All simulations are conducted for $a=900, b=1.5, m=0.1$ and $\bar{c}=500$.
} 
positive; in Bertrand with linear demand the strategic effect is always negative. Finally, in line with the simultaneous case, $\lambda_{\mathrm{TS}}^{o}$ and $\lambda_{\mathrm{CS}}^{o}$ decrease with $\gamma$. Note also that we do not have a bang-bang solution for CS.

Optimal degree of overlapping ownership (TS and CS standard) ${ }^{18}$

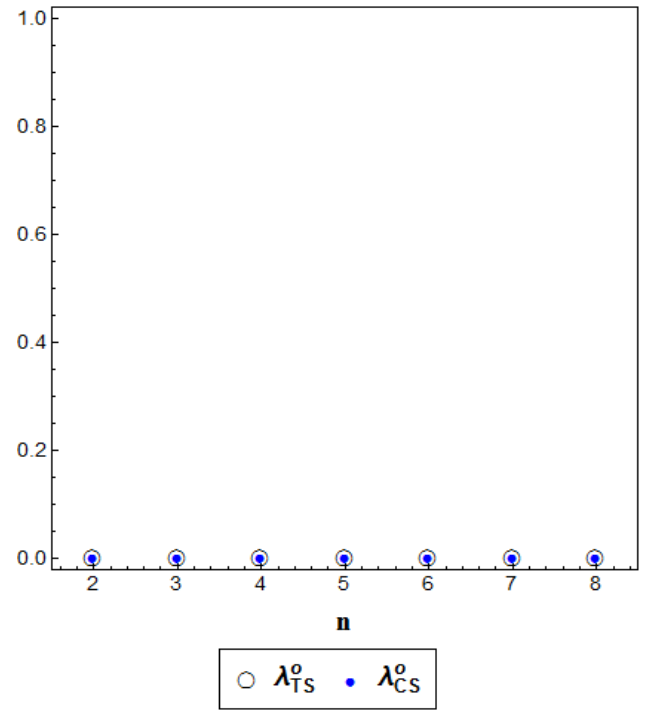

Fig. B9a. Linear Bertrand two-stage model. $(\gamma=80, \beta=0.2)$

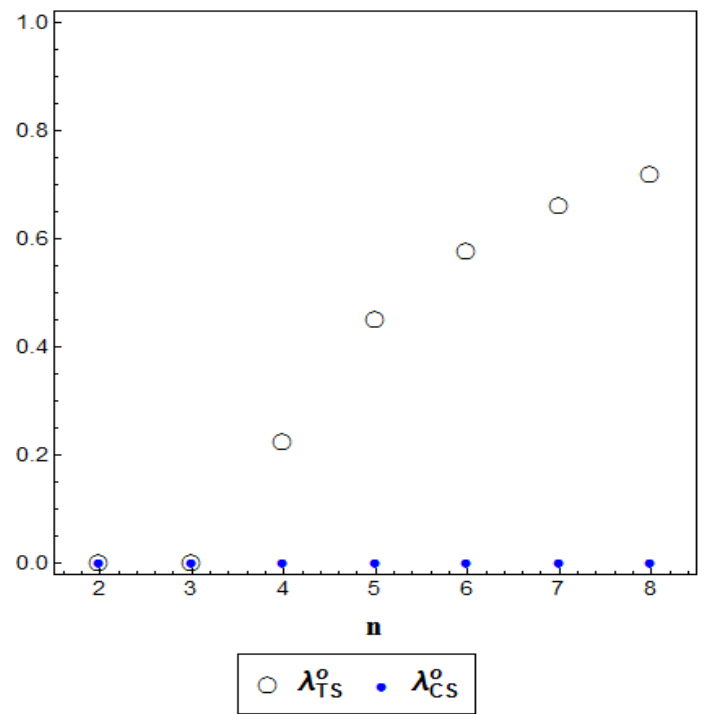

Fig. B9c. Linear Bertrand two-stage model. $(\gamma=80, \beta=0.6)$

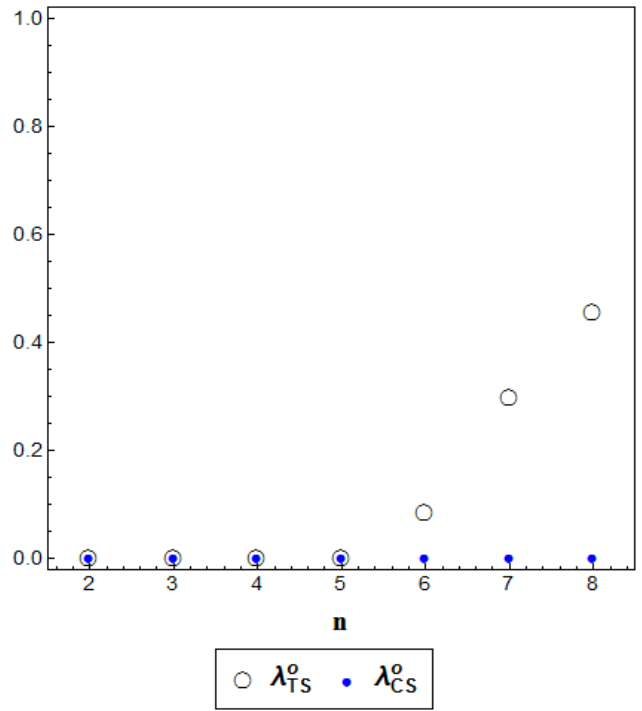

Fig. B9b. Linear Bertrand two-stage model. $(\gamma=80, \beta=0.4)$

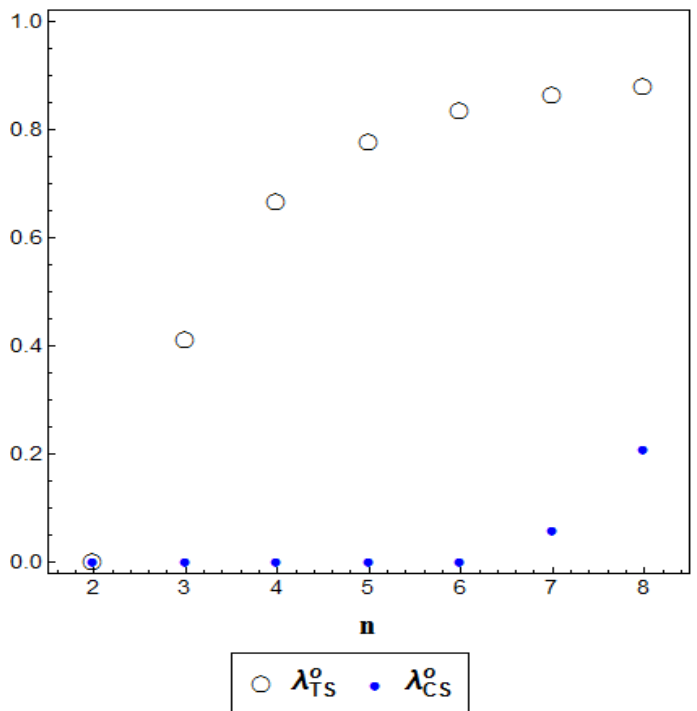

Fig. B9d. Linear Bertrand two-stage model. $(\gamma=80, \beta=0.8)$

Figures B9a-d confirm that it is not optimal to allow overlapping ownership for highly concentrated markets. In line with the other models, $\lambda_{\text {TS }}^{o}$ weakly increases with the number of firms, and $\lambda_{\mathrm{CS}}^{o}$ increases weakly with $n$ (only if $n$ is sufficiently large given the size of the

\footnotetext{
${ }^{18}$ All simulations are conducted for $a=900, b=1.5, m=0.1$ and $\bar{c}=500$.
} 
spillover).

Optimal degree of overlapping ownership (TS and CS standard) ${ }^{19}$

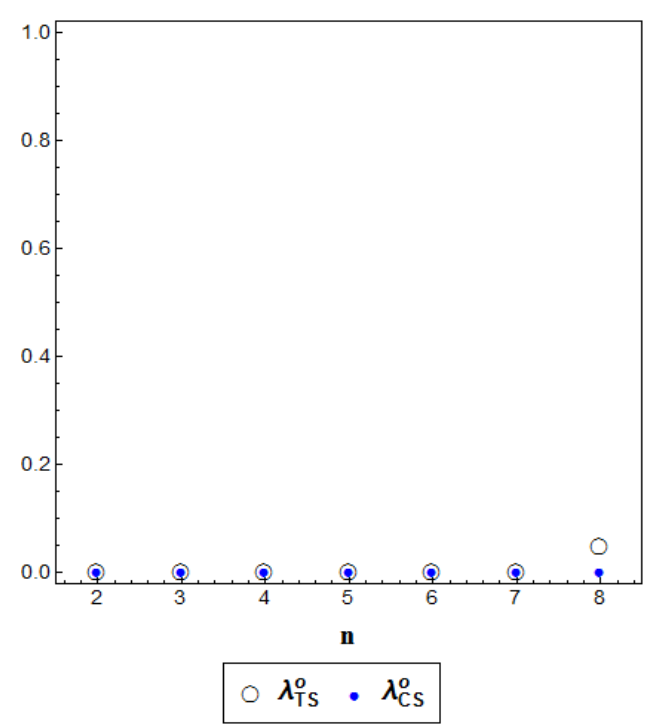

Fig. B10a. Linear Bertrand two-stage model. $(\gamma=60, \beta=0.2)$

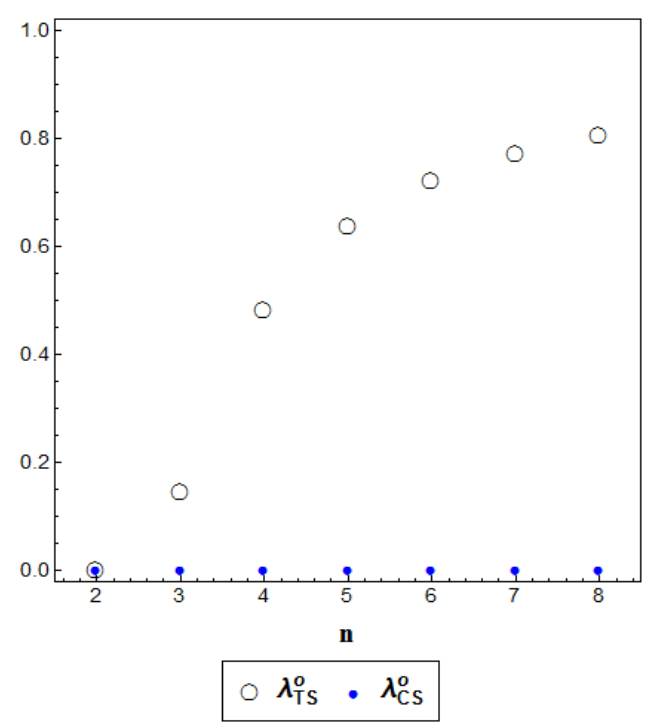

Fig. B10c. Linear Bertrand two-stage model. $(\gamma=60, \beta=0.6)$

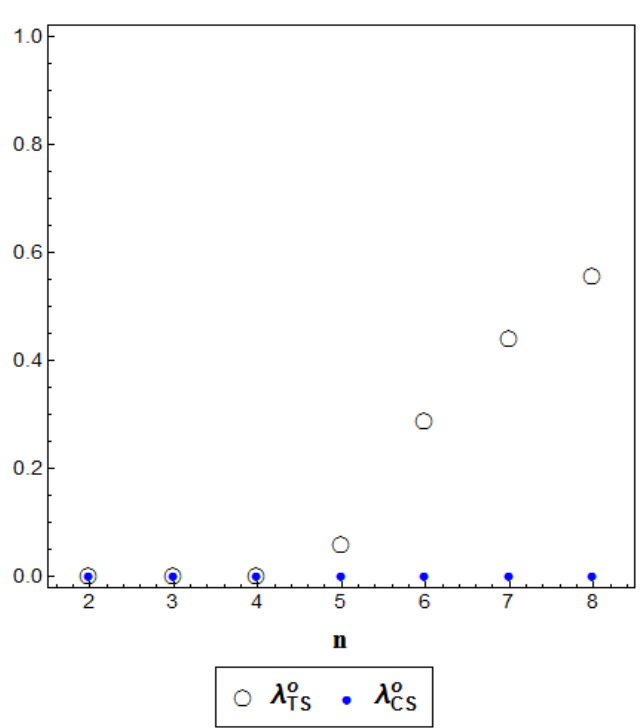

Fig. B10b. Linear Bertrand two-stage model. $(\gamma=60, \beta=0.4)$

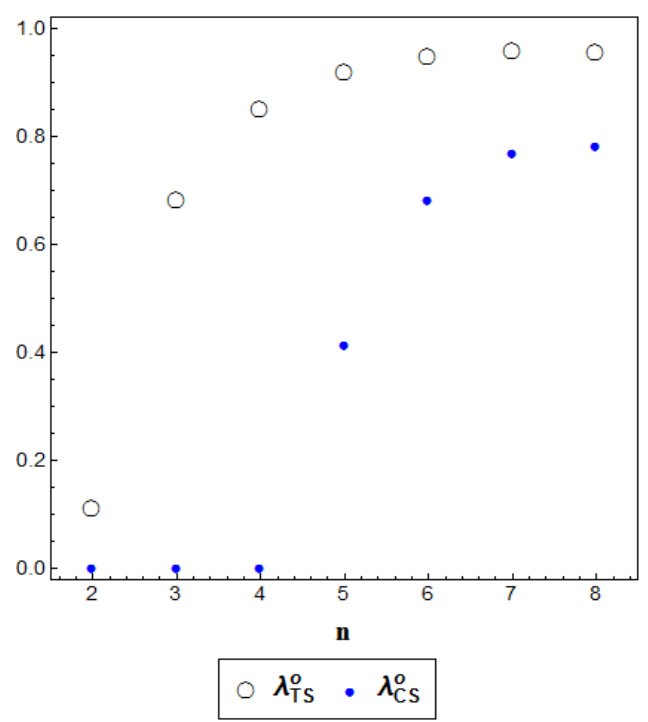

Fig. B10d. Linear Bertrand two-stage model. $(\gamma=60, \beta=0.8)$

\footnotetext{
${ }^{19}$ All simulations are conducted for $a=900, b=1.5, m=0.1$ and $\bar{c}=500$.
} 


\section{B.4.2 Constant elasticity model}

Model specification: main assumptions. Consider the following form for the representative consumer's utility function

$$
U=\left[\sum_{i=1}^{n} q_{i}^{\rho}\right]^{1 / \rho} q_{0}^{\theta}
$$

with $\rho \in(0,1)$ and $\theta>0$, and where $q_{0}$ is the numéraire and $q_{i}$ is quantity for the variety $i$ of the differentiated product. The consumer's problem consists of maximizing $U$ subject to the budget constraint $\sum_{i=0}^{n} p_{i} q_{i}=Y$, where $Y$ is aggregate income. The demand functions resulting from this problem are

$$
D_{i}(\mathbf{p})=\frac{p_{i}^{-1-1 / \mu}}{\sum_{j=1}^{n} p_{j}^{-1 / \mu}} S,
$$

where $\mu=(1-\rho) / \rho \in(0, \infty)$, and $S \equiv Y /(1+\theta)$ is the total spending on the differentiated product variants; the amount of numéraire is $q_{0}=\theta S$. Note that $\sigma=1 /(1-\rho)$ is the constant elasticity of substitution between any two products. As $\rho \rightarrow 1(\sigma \rightarrow \infty)$, products become perfect substitutes, while as $\rho \rightarrow 0(\sigma \rightarrow 1)$, products become independent.

The innovation function is $c_{i}=\kappa\left(x_{i}+\beta \sum_{j \neq i} x_{j}\right)^{-\alpha}$ with $\alpha, \kappa>0$, whereas the investment cost function is $\Gamma\left(x_{i}\right)=x_{i}$. Thus, the innovation and investment functions satisfy Assumptions A.2 and A.3. CE demand, as specified, is not quasilinear, but it is smooth and downward sloping, the demand system is symmetric and products are gross substitutes (Assumption 1B). From Table B3, we get at the symmetric equilibrium

$$
v^{*}=-\frac{S}{n p^{* 2}}<0, \text { and } v_{\lambda}^{*}=-\frac{(n-1)(1-\lambda)+\mu n}{n^{2} p^{* 2} \mu} S<0 .
$$

Table B3: CE Demand Bertrand Basic Derivatives

\begin{tabular}{l||c}
\multicolumn{1}{c}{ for $i \neq j, j \neq k, i \neq k$} \\
\hline$\partial D_{i}\left(p^{*}\right) / \partial p_{i}=$ & $-\frac{S}{n^{2} p^{* 2} \mu}(n-1+n \mu)$ \\
$\partial^{2} D_{i}\left(p^{*}\right) / \partial p_{i}{ }^{2}=$ & $\frac{2 S}{n^{3} p^{* 3} \mu^{2}}\left[n^{2} \mu^{2}+\frac{3}{2}(n-1) n \mu+\frac{1}{2}(n-2)(n-1)\right]$ \\
$\partial D_{i}\left(p^{*}\right) / \partial p_{j}=$ & $\frac{S}{n^{2} p^{* 2} \mu}$ \\
$\partial D_{i}\left(p^{*}\right) / \partial p_{j}{ }^{2}=$ & $-\frac{S}{n^{3} p^{* 3} \mu^{2}}[(n-2)+n \mu]$ \\
$\partial^{2} D_{i}\left(p^{*}\right) / \partial p_{j} \partial p_{i}=$ & $-\frac{S}{n^{3} p^{* 3} \mu^{2}}[(n-2)+n \mu]$ \\
$\partial^{2} D_{i}\left(p^{*}\right) / \partial p_{k} \partial p_{j}=$ & $\frac{2 S}{n^{3} p^{* 3} \mu^{2}}$ \\
\hline
\end{tabular}

Simultaneous model. Interior equilibrium. The FOCs in the symmetric solution are given by (61) and (62):

$$
\frac{p^{*}-c\left(B x^{*}\right)}{p^{*}}=\frac{1}{\eta_{i}-\lambda(n-1) \eta_{i k}}
$$




$$
-c^{\prime}\left(B x^{*}\right) q^{*} \tau=\Gamma^{\prime}\left(x^{*}\right),
$$

where $\eta_{i}=(n-1+n \mu) / n \mu$ and $\eta_{i k}=1 / n \mu$. In the symmetric solution: $D_{i}=D_{k}=q^{*}=$ $S /\left(n p^{*}\right), c_{i}=\kappa\left(B x^{*}\right)^{-\alpha}, c^{\prime}=\partial c_{i} /\left.\partial x_{i}\right|_{x_{i}=x^{*}}=-\alpha \kappa\left(B x^{*}\right)^{-\alpha-1}$, and $\Gamma^{\prime}\left(x^{*}\right)=1$; by solving the system of FOCs for $p^{*}$ and $x^{*}$ we get the symmetric interior equilibrium:

$$
\begin{gathered}
x^{*}=\frac{\alpha \tau S A}{B n} \\
p^{*}=\frac{\kappa}{A(\alpha \tau S A / n)^{\alpha}},
\end{gathered}
$$

where

$$
A=1+\frac{\mu n}{\Lambda-n(1+\mu)}=\frac{n-\Lambda}{n-\Lambda+n \mu}>0 \text { for } \lambda<1
$$

Table B4: CE Bertrand Model

\begin{tabular}{l||c}
\hline Demand & $D_{i}(p)=S p_{i}^{-1-1 / \mu} / \sum_{j=1}^{n} p_{j}^{-1 / \mu}$ \\
$c_{i}=$ & $\kappa\left(x_{i}+\beta \sum_{j \neq i} x_{j}\right)^{-\alpha}$ \\
$\Gamma(x)=$ & $x$ \\
$v=$ & $-S / n p^{* 2}$ \\
$v_{\lambda}=$ & $-S[(n-1)(1-\lambda)+n \mu] / n^{2} p^{* 2} \mu$ \\
S.O.C & $\tau n^{4} \mu(n-\Lambda) \tilde{\lambda}(1+\alpha)(1+\mu)-n^{2} \alpha A(\mu n+n-\Lambda)[(1+\mu) n-\tau]^{2}>0$ \\
Regularity Condition & $\lambda<1$ \\
\hline \hline
\end{tabular}

with $\tilde{\lambda}=1+\lambda(n-1) \beta^{2}$.

Second-order, stability and regularity conditions. We first check the stability and regularity conditions; using (65) and (70) and from Table B3 we obtain

$$
\begin{gathered}
\Delta_{x}=-(1+\alpha) \frac{B n}{\alpha \tau S A}<0, \\
\Delta_{p}=-\frac{(1-\lambda)(n-1)}{n^{2} p^{* 2} \mu} S<0
\end{gathered}
$$

and

$$
\begin{aligned}
\Delta & =\Delta_{p} \Delta_{x}-\tau B v v_{\lambda}\left(c^{\prime}\left(B x^{*}\right)\right)^{2} \\
& =\frac{A\left(\frac{\alpha \tau S A}{n}\right)^{2 \alpha} B}{n \kappa^{2} \mu \alpha \tau}(n-1)(1-\lambda)>0
\end{aligned}
$$

for $\lambda<1$.

Second order conditions are: (i) $\partial_{p_{i} p_{i}} \phi_{i}<0$; (ii) $\partial_{x_{i} x_{i}} \phi_{i}<0$; and (iii) $\partial_{p_{i} p_{i}} \phi_{i}\left(\partial_{x_{i} x_{i}} \phi_{i}\right)-$ 
$\left(\partial_{p_{i} x_{i}} \phi_{i}\right)^{2}>0$. Conditions (i) and (ii) are satisfied:

$$
\begin{aligned}
\partial_{p_{i} p_{i}} \phi_{i} & =2 \frac{\partial D_{i}\left(p^{*}\right)}{\partial p_{i}}-\left(\frac{q^{*}}{v_{L}}\right)\left[\frac{\partial^{2} D_{i}\left(p^{*}\right)}{\partial p_{i}^{2}}+\lambda(n-1) \frac{\partial^{2} D_{k}\left(p^{*}\right)}{\partial p_{i}^{2}}\right] \\
& =-\frac{S^{1+2 \alpha}\left(\frac{\alpha \tau}{n}\right)^{2 \alpha} A^{2 \alpha+2}(1-\lambda)(1+\mu)\left(\frac{n-1}{n}\right)}{[(1-\lambda)(n-1)+\mu n] \mu \kappa^{2}}<0
\end{aligned}
$$

and

$$
\begin{aligned}
\partial_{x_{i} x_{i}} \phi_{i} & =-c^{\prime \prime}\left(B x^{*}\right)\left[1+\lambda(n-1) \beta^{2}\right] q^{*}-\Gamma^{\prime \prime}\left(x^{*}\right) \\
& =-\left(\frac{1+\alpha}{\alpha}\right) \frac{n}{\tau S A}\left[1+\lambda(n-1) \beta^{2}\right]<0 .
\end{aligned}
$$

Using that

$$
\partial_{p_{i} x_{i}} \phi_{i}=-\frac{\left(\frac{\alpha \tau S A}{n}\right)^{\alpha} A}{\kappa n \mu \tau}[(n-1)(1-\beta \lambda)+n \mu],
$$

we have that condition (iii) is satisfied iff

$$
-\frac{\left(\frac{\alpha \tau S A}{n}\right)^{2 \alpha} A}{\mu^{2} n^{4}[(n-1)(1-\lambda)+\mu n] \kappa^{2} \tau \alpha}\{D-E\}>0
$$

where $D \equiv A^{3}[(-\beta \lambda+\mu+1) n+\lambda \beta-1]^{2}[(n-1)(1-\lambda)+\mu n] \tau S^{2} \alpha^{3} \frac{n^{2}}{(\alpha \tau S A)^{2}}$ and $E \equiv n^{4} \mu(1-$ $\lambda)(n-1)\left[1+\lambda(n-1) \beta^{2}\right](1+\alpha)(1+\mu)$. Therefore, the SOC reduces to

$$
\tau n^{4} \mu(n-\Lambda) \tilde{\lambda}(1+\alpha)(1+\mu)-n^{2} \alpha A(\mu n+n-\Lambda)[(1+\mu) n-\tau]^{2}>0,
$$

where $\tilde{\lambda} \equiv 1+\lambda(n-1) \beta^{2}$.

Comparative statics on $\lambda$ and spillover thresholds. Recall that only $R_{\mathrm{I}}$ exits (irrespective of the spillover level) if $-\Delta_{p}<\left(\partial D_{k}\left(p^{*}\right) / \partial p_{i}\right) \Lambda\left(v / v_{\lambda}\right)$; replacing terms and simplifying the condition reduces to

$$
\mu n(2 \Lambda-n)-(n-1)^{2}(1-\lambda)^{2}>0,
$$

which holds for $\lambda=1$. If (103) does not hold, then we may identify $R_{\mathrm{II}}$ and $R_{\mathrm{III}}$ by deriving the corresponding spillover threshold. From (99) we have that

$$
\frac{\partial x^{*}}{\partial \lambda}=\frac{2 \alpha S(n-1)}{B n[n-\Lambda+\mu n]^{2}}\left\{-\left[\left(-\frac{\lambda^{2}}{2}+(1+\mu) \lambda-\frac{1+\mu}{2}\right) n+\frac{(1-\lambda)^{2}}{2}\right] \beta(n-1)-\frac{\mu n}{2}\right\},
$$

which implies that

$$
\operatorname{sign}\left\{\frac{\partial x^{*}}{\partial \lambda}\right\}=\operatorname{sign}\left\{\beta\left[\left(\frac{\lambda^{2}}{2}-(1+\mu) \lambda+\frac{1+\mu}{2}\right) n-\frac{(1-\lambda)^{2}}{2}\right](n-1)-\frac{\mu n}{2}\right\} .
$$


Therefore,

$$
\text { if } \beta \leq \underline{\beta}(\lambda) \equiv \frac{\mu n}{(n-1)\left\{\left[\lambda^{2}+(1+\mu)(1-2 \lambda)\right] n-(1-\lambda)^{2}\right\}},
$$

then $\partial x^{*} / \partial \lambda \leq 0$ and $\partial p^{*} / \partial \lambda>0\left(R_{\mathrm{I}}\right)$. Simple calculations show that $\partial \underline{\beta}(\lambda) / \partial \mu>0$. Since $d \mu / d \rho=-1 / \rho^{2}<0$, we have that $\partial \underline{\beta}(\lambda) / \partial \rho<0$. From (100) we obtain

$$
\frac{\partial p^{*}}{\partial \lambda}=\frac{2 \kappa}{(n-1)(1-\lambda)^{2}\left\{\frac{(n-1)(1-\lambda) \alpha S \tau}{n[\mu n+(n-1)(1-\lambda)]}\right\}^{\alpha} \tau} \vartheta_{\mathrm{CE}}
$$

where

$$
\vartheta_{\mathrm{CE}} \equiv(n-1)\left\{\left[\left(-\frac{\lambda^{2}}{2}+(\mu+1) \lambda-\frac{1+\mu}{2}\right) n+\frac{(1-\lambda)^{2}}{2}\right] \alpha+\frac{\lambda \mu n}{2}\right\} \beta+\frac{\mu n(1+\alpha)}{2} .
$$

It follows that

$$
\operatorname{sign}\left\{\frac{\partial p^{*}}{\partial \lambda}\right\}=\operatorname{sign}\left\{\vartheta_{\mathrm{CE}}\right\}
$$

Consequently,

$$
\beta^{\prime}=\frac{\mu n(1+\alpha)}{(n-1)\left(\left\{\left[\lambda^{2}+(1+\mu)(1-2 \lambda)\right] n-(1-\lambda)^{2}\right\} \alpha-\lambda \mu n\right)} .
$$

Using that $\Gamma^{\prime \prime}=0$ and by replacing $p^{*}, x^{*}, \partial D_{k}\left(p^{*}\right) / \partial p_{i}, c^{\prime}\left(B x^{*}\right), c^{\prime \prime}\left(B x^{*}\right)$ and $\Gamma^{\prime}\left(x^{*}\right)$ into $(75)$ we obtain:

$$
H=\frac{n \mu(1+\alpha) \tau B}{(n-\Lambda) \alpha[n(1+\mu)-\Lambda]} .
$$

Note that $\operatorname{sign}\left\{\partial p^{*} / \partial \lambda\right\}=\operatorname{sign}\{H-\beta B\}$, so by solving $H-\beta B=0$ for $\beta$ we obtain again the expression for $\beta^{\prime}$ given by (105).

Recall that $C S^{\prime}(\lambda)>(<) 0$ iff $\beta>(<) \beta^{\prime}$. The threshold $\beta^{\prime}$ is strictly increasing in $\lambda$ :

$$
\frac{\partial \beta^{\prime}}{\partial \lambda}=\frac{\mu n(1+\alpha)}{(n-1)} \frac{[\mu n(1+2 \alpha)+2(1-\lambda)(n-1) \alpha]}{\left(\left\{\left[\lambda^{2}+(1+\mu)(1-2 \lambda)\right] n-(1-\lambda)^{2}\right\} \alpha-\lambda \mu n\right)^{2}}>0 .
$$

As a result, $\lambda_{\mathrm{CS}}^{o}>0$ if $\beta>\beta^{\prime}(0)$, where

$$
\beta^{\prime}(0)=\frac{\mu n(1+\alpha)}{(n-1)[(1+\mu) n-1] \alpha} .
$$


Spillover thresholds and regions $R_{\mathrm{I}}, R_{\mathrm{II}}$ and $R_{\mathrm{III}}{ }^{20}$

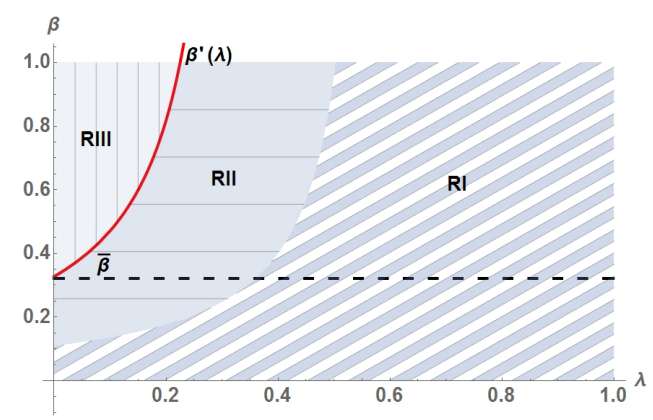

Fig. B11a. CE Bertrand model.

$$
(n=6, \alpha=0.5, \rho=0.5)
$$

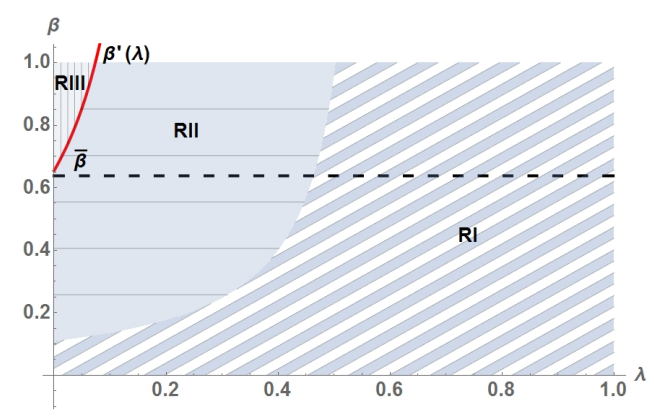

Fig. B11c. CE Bertrand model.

$$
(n=6, \alpha=0.2, \rho=0.5)
$$

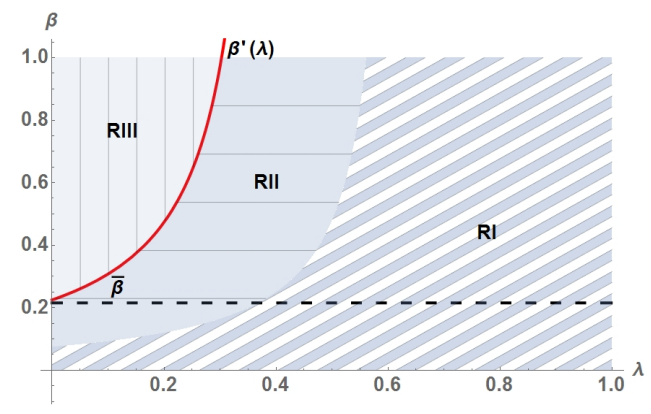

Fig. B11e. CE Bertrand model.

$$
(n=6, \alpha=0.5, \rho=2 / 3)
$$

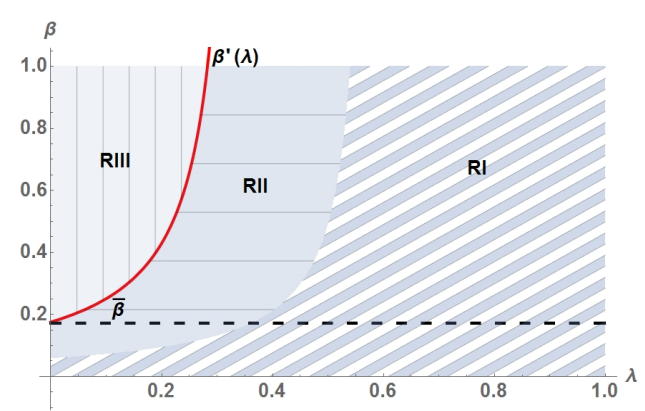

Fig. B11b. CE Bertrand model.

$$
(n=10, \alpha=0.5, \rho=0.5)
$$

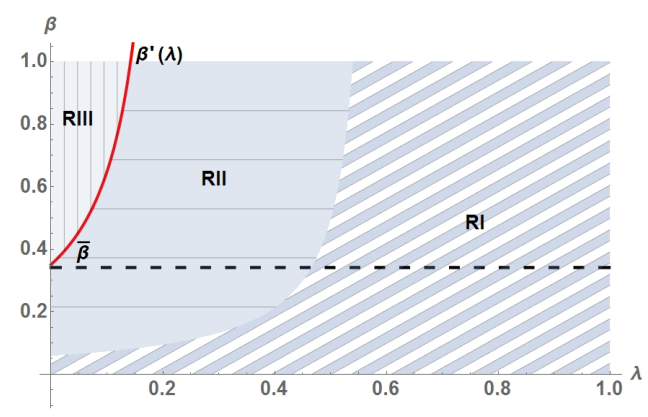

Fig. B11d. CE Bertrand model.

$$
(n=10, \alpha=0.2, \rho=0.5)
$$

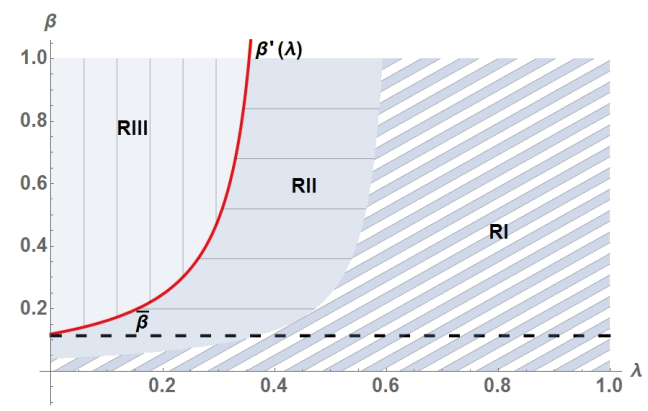

Fig. B11f. CE Bertrand model.

$$
(n=10, \alpha=0.5, \rho=2 / 3)
$$

We depict the spillover thresholds and the three regions in Figures B11a-f. For illustrative

\footnotetext{
${ }^{20}$ All simulations are conducted for $\kappa=1, Y=20$ and $\theta=0.05$. Note that $S=Y /(1+\theta)$.
} 
purposes, we consider six cases that differ in $n, \alpha$ and $\rho$. In contrast to the linear demand case, the condition under which only $R_{\mathrm{I}}$ exists for all $\beta$, which is given by (103), always holds for $\lambda$ close or equal to 1 . For lower values of $\lambda, R_{\mathrm{II}}$ and/or $R_{\mathrm{III}}$ may exist for $\beta$ sufficiently high. Fig. 11a-d show how area $R_{\mathrm{III}}$ (respectively, $R_{\mathrm{II}}$ ) increases (decreases) with $\alpha$, and illustrate that $R_{\mathrm{III}}$ increases with $n$. Finally, the comparison of Fig. B11a with B11e, and B11b with B11f, display the increase of $R_{\text {III }}$ with $\rho$.

Comparative statics on $\beta^{\prime}$. Straightforward calculations show that $\beta^{\prime}(\lambda)$ is strictly decreasing in $\alpha$ and strictly increasing in $\mu$. Thus, $\partial \beta^{\prime}(\lambda) / \partial \rho<0$. As in the linear demand case, $\beta^{\prime}(0)$ is strictly decreasing in $n$. Therefore, if $\beta^{\prime}(0)>1$ for $n=2$, which holds when $\mu>\alpha / 2$, then to have $\beta^{\prime}(0)<1$, so that $\lambda_{\mathrm{CS}}^{o}>0$ when $\beta>\beta^{\prime}(0)$, the number of firms must be sufficiently high such that

$$
n>\frac{2(1+\mu) \alpha+\mu+2 \sqrt{\left[\left(\alpha+\frac{1}{2}\right)^{2} \mu+\alpha^{2}+\alpha\right] \mu}}{2(1+\mu) \alpha} .
$$

PROPOSITION BCE1 Under the CE demand specification, if $\mu n(2 \Lambda-n)-(n-1)^{2}(1-\lambda)^{2}>0$ then only region $R_{\mathrm{I}}$ exists. Otherwise, assume $n>H(1)$, where $H$ is given by (106), and let $\underline{\beta}(\lambda)$ and $\beta^{\prime}$ be given, respectively, by (104) and (105). Then the following statements hold:

(i) if $\beta \leq \underline{\beta}(\lambda)$, then $\frac{\partial q^{*}}{\partial \lambda}<0$ and $\frac{\partial x^{*}}{\partial \lambda} \leq 0\left(R_{\mathrm{I}}\right)$;

(ii) if $\underline{\beta}(\lambda)<\beta \leq \beta^{\prime}$, then $\frac{\partial q^{*}}{\partial \lambda} \leq 0$ and $\frac{\partial x^{*}}{\partial \lambda}>0\left(R_{\mathrm{II}}\right)$;

(iii) if $\beta>\beta^{\prime}$, then $\frac{\partial q^{*}}{\partial \lambda}>0$ and $\frac{\partial x^{*}}{\partial \lambda}>0\left(R_{\mathrm{III}}\right)$.

We have that $\beta(\lambda)$ and $\beta^{\prime}(\lambda)$ are increasing in $\lambda$ and decreasing in $\rho$, and $\partial \beta^{\prime}(0) / \partial n<0$.

Profit. By inserting equilibrium values into the profit function and simplifying, we obtain:

$$
\pi(\lambda)=\frac{1}{n B}\left[\frac{n \mu B-\alpha \tau(n-\Lambda)}{(\mu+1) n-\Lambda}\right] S
$$

Simulations show that also in Bertrand with CE demand, profit in equilibrium is strictly increasing in the degree of overlapping ownership: $\pi^{* \prime}(\lambda)>0$.

Utility. Note that the indirect utility function in not linear in income. Thus, to solve the first-best problem we have to maximize the utility function subject to the resource constraint: $Y=\sum_{i=1}^{n} c_{i} q_{i}+\sum_{i=1}^{n} \Gamma\left(x_{i}\right)+q_{0}$. At the symmetric equilibrium the utility function with this constraint included is

$$
V(\lambda)=n^{1 / \rho} q^{*}\left(Y-n c\left(B x^{*}\right) q^{*}-n x^{*}\right)^{\theta},
$$


where $Y=S(1+\theta)$. Computing $V^{\prime}(\lambda)$ and using the FOC $1=-c^{\prime}\left(B x^{*}\right) \tau q^{*}$, after some manipulations we can write

$V^{\prime}(\lambda)=n^{1 / \rho} \varrho^{\theta-1}\left\{\left[S(1+\theta)-n c\left(B x^{*}\right) q^{*}(1+\theta)-n x^{*}\right] \frac{\partial q^{*}}{\partial \lambda}-c^{\prime}\left(B x^{*}\right) \beta(n-1)(1-\lambda) \theta n q^{*^{2}} \frac{\partial x^{*}}{\partial \lambda}\right\}$,

where $\varrho \equiv S(1+\theta)-n c\left(B x^{*}\right) q^{*}-n x^{*}$.

We now may obtain the threshold $\bar{\beta}$ from the condition $W^{\prime}(0)>0$. In particular, the equation $W^{\prime}(0)=0$ is quadratic in $\beta$, and writes as $\vartheta_{1} \beta^{2}+\vartheta_{2} \beta+\vartheta_{3}=0$, where

$$
\begin{aligned}
\vartheta_{1} & \equiv \alpha[(\mu+1) n-1](n-1)^{2}\left\{S \alpha \theta(n-1)^{2} Z^{-1}+[(\mu+1) n-1] n(1+\theta) n \mu\right\}, \\
\vartheta_{2} \equiv & -n(n-1)\left\{-[(\mu+1) n-1](1+\theta)[-(n-1) \alpha+n \mu](n-1)+S \mu \alpha^{2} \theta(n-1)^{2} Z^{-1}\right. \\
& \left.+[(\mu+1) n-1]^{2}\left[(n-1) \alpha^{2}-(1+\theta)(n-1) \alpha+n \mu(1+\theta)\right]\right\},
\end{aligned}
$$

and

$$
\vartheta_{3} \equiv-n^{2} \mu(\alpha+1)[(\mu+1) n-1]\{[(1+\theta) \mu-\alpha] n+\alpha\}
$$

with

$$
Z \equiv \frac{\alpha S(n-1)}{[(\mu+1) n-1] n}
$$

The threshold $\bar{\beta}$ is given by the positive root:

$$
\bar{\beta}=\frac{-\vartheta_{2}+\sqrt{\vartheta_{2}^{2}-4 \vartheta_{1} \vartheta_{3}}}{2 \vartheta_{1}} .
$$

Table B5: $H$ and Spillover Thresholds in CE Bertrand Model

\begin{tabular}{l||c}
\hline$H=$ & $n \mu(1+\alpha) \tau B /\{(n-\Lambda) \alpha[n(1+\mu)-\Lambda]\}$ \\
$\beta(\lambda)=$ & $\mu n /\left((n-1)\left\{\left[\lambda^{2}+(1+\mu)(1-2 \lambda)\right] n-(1-\lambda)^{2}\right\}\right)$ \\
$\bar{\beta}=$ & $\left(-\vartheta_{2}+\sqrt{\vartheta_{2}^{2}-4 \vartheta_{1} \vartheta_{3}}\right) /\left(2 \vartheta_{1}\right)$ \\
$\beta^{\prime}=$ & $\mu n(1+\alpha) /\left[(n-1)\left(\left\{\left[\lambda^{2}+(1+\mu)(1-2 \lambda)\right] n-(1-\lambda)^{2}\right\} \alpha-\lambda \mu n\right)\right]$ \\
\hline
\end{tabular}


Threshold value $\bar{\beta}$

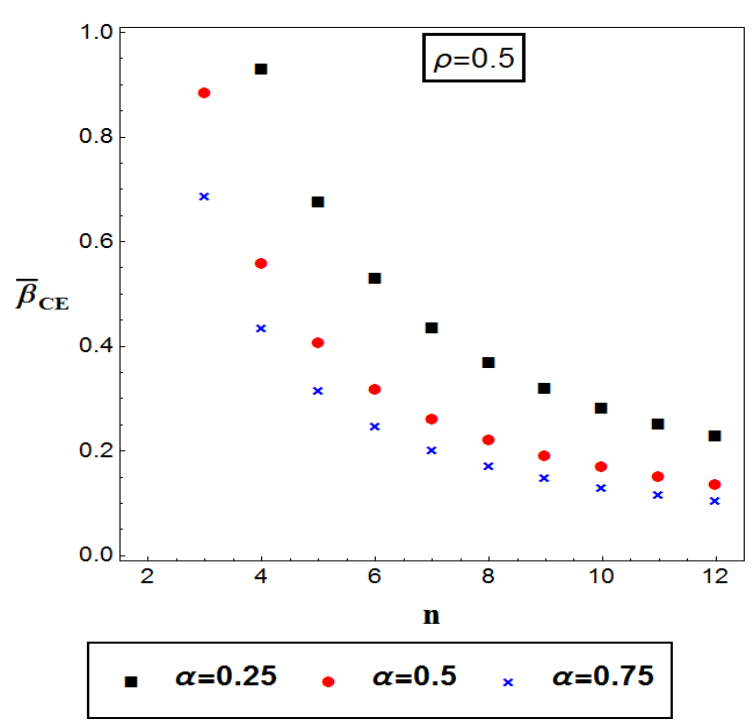

Fig. B12a.CE Bertrand model.

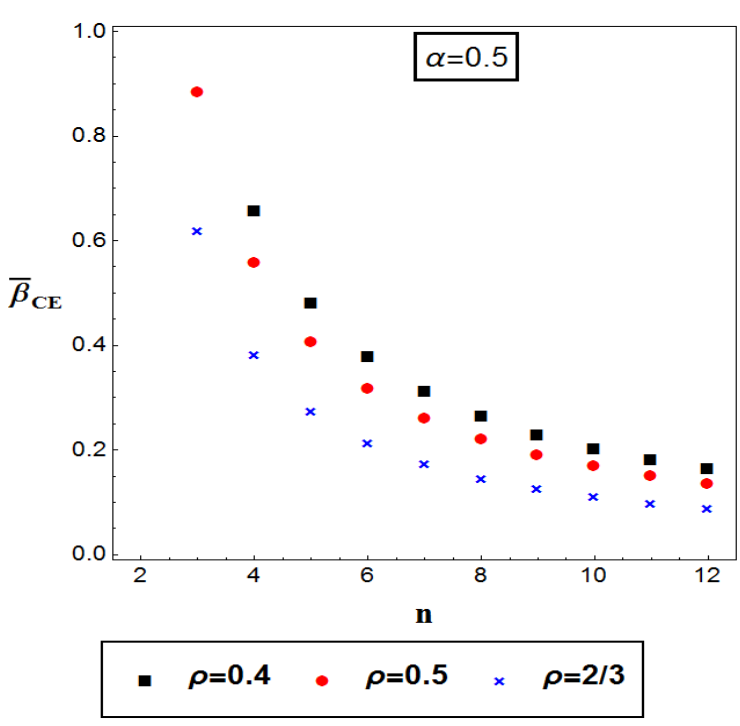

Fig. B12b. CE Bertrand model.

Comparative statics on $\bar{\beta}$. We observe in Fig. 12a,b that $\bar{\beta}$ decreases with $\alpha$ and $\rho$. The threshold as in the previous cases decreases with $n$ and may take values greater than 1 (so $\lambda_{\mathrm{TS}}^{o}=0$ irrespective of the value of $\beta$ ) when there are few firms in the market. Note that we use notation $\lambda_{\text {TS }}^{o}$ with subscript TS even though we refer to utility $V$.

Comparative statics on the socially optimal degree of overlapping ownership. Simulation results are in line with previous findings: the socially optimal level of overlapping ownership increases with the size of spillovers (see Figures B13a-d) and with the number of firms (see Figures B14a-d). 
Optimal degree of overlapping ownership (TS and CS standard) ${ }^{21}$

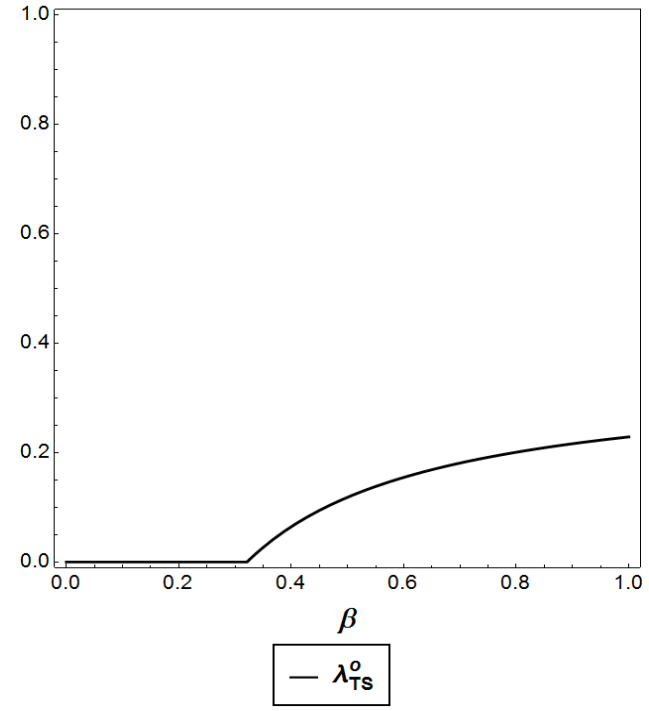

Fig. B13a. CE Bertrand model.

$$
(\alpha=0.5, \rho=0.5, n=6)
$$

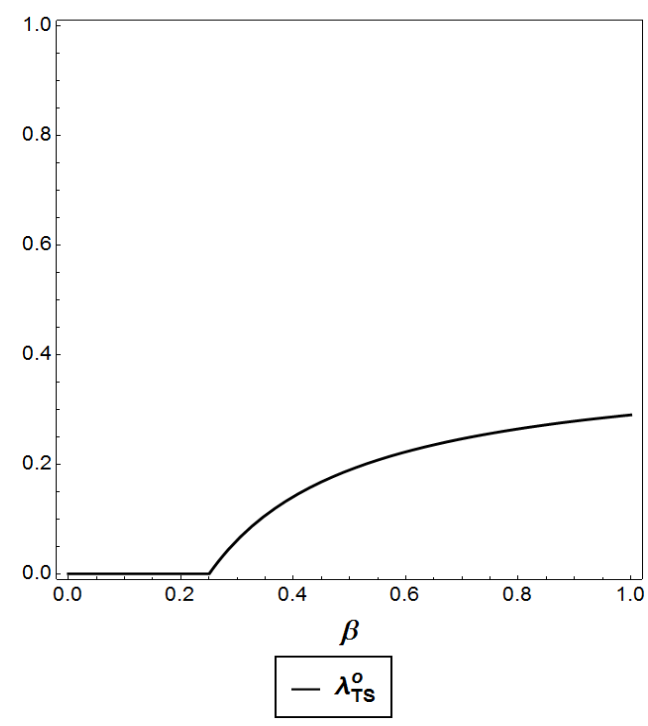

Fig. B13c. CE Bertrand model.

$$
(\alpha=0.75, \rho=0.5, n=6)
$$

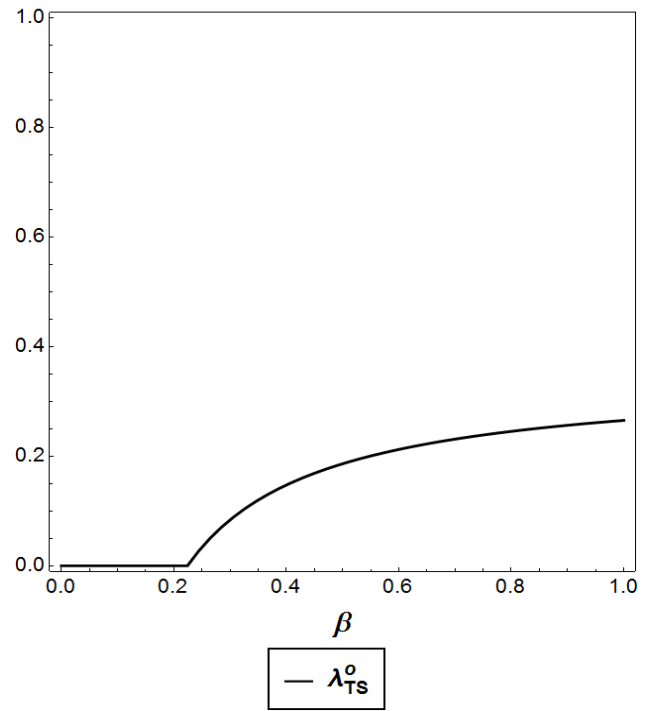

Fig. B13b. CE Bertrand model.

$$
(\alpha=0.5, \rho=0.5, n=8)
$$

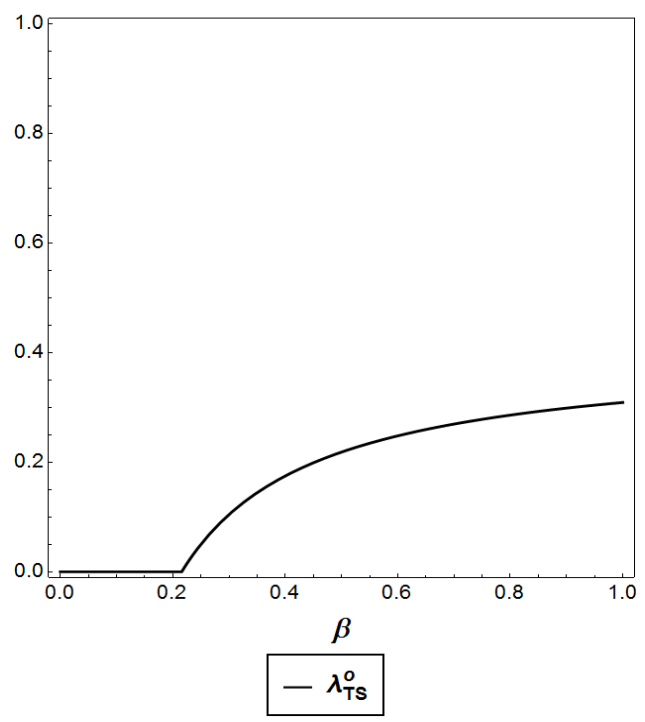

Fig. B13d. CE Bertrand model.

$$
(\alpha=0.5, \rho=2 / 3, n=6)
$$

\footnotetext{
${ }^{21}$ All simulations are conducted for $\kappa=1, Y=20$ and $\theta=0.05$.
} 
Optimal degree of overlapping ownership (TS and CS standard) ${ }^{22}$

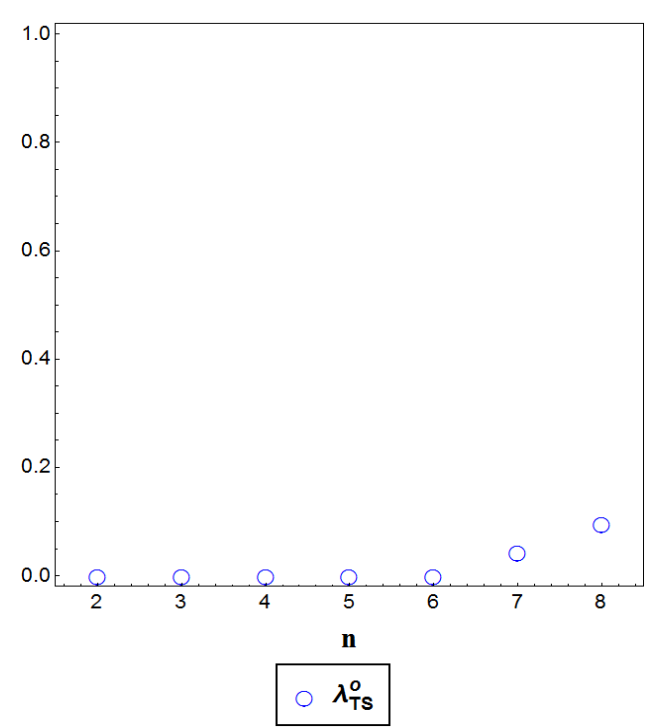

Fig. B14a. CE Bertrand model.

$$
(\alpha=0.5, \rho=2 / 3, \beta=0.2 \text {. })
$$

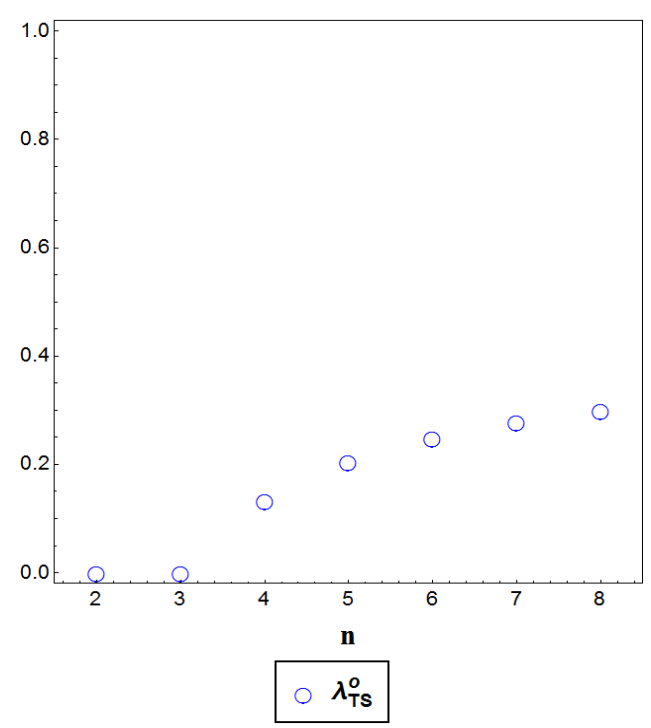

Fig. B14c. CE Bertrand model.

$$
(\alpha=0.5, \rho=2 / 3, \beta=0.6 \text {. })
$$

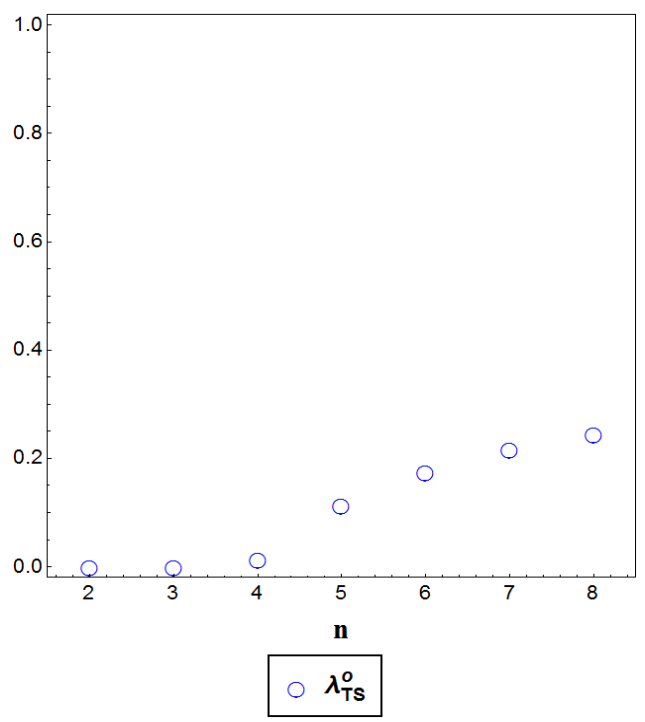

Fig. B14b.CE Bertrand model. $(\alpha=0.5$, $\rho=2 / 3, \beta=0.4$.)

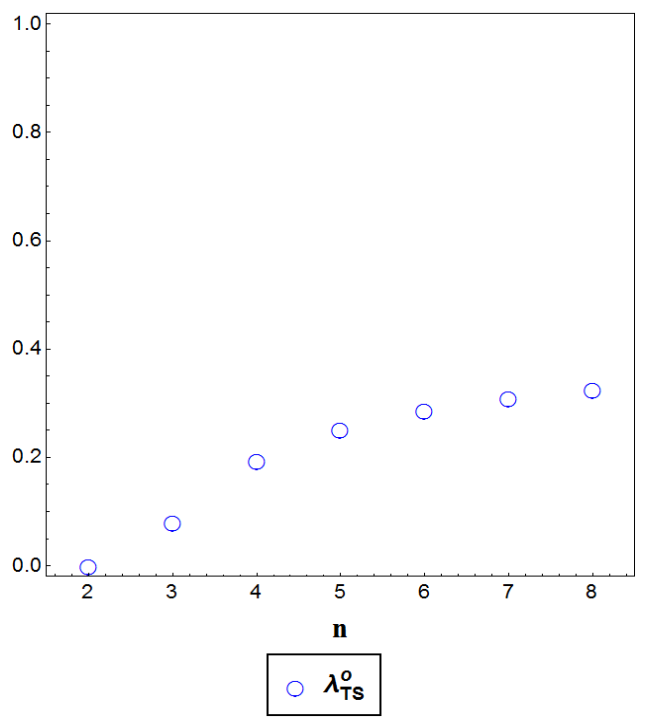

Fig. B14d. CE Bertrand model.

$$
(\alpha=0.5, \rho=2 / 3, \beta=0.8 \text {.) }
$$

Comparative statics on the degree of product differentiation. In Fig. B15a-d we depict the optimal degree of overlapping ownership $\lambda_{\mathrm{TS}}^{o}$ for $\rho \in(0,1)$; if $\rho \rightarrow 0^{+}$, then products tend to be independent, while if $\rho \rightarrow 1^{-}$, then products tend to be perfect substitutes. The grey area represents the values for $\rho$ and $\lambda$ where the interior (regular) equilibrium exists. ${ }^{23}$ Simulations

\footnotetext{
${ }^{22}$ All simulations are conducted for $\kappa=1, Y=20$ and $\theta=0.05$.

${ }^{23}$ That is, the second-order condition holds, and profit, cost, price, output and R\&D are positive. (The regularity condition holds for $\lambda<1$.)
} 
show that for $\beta>0, \lambda_{\mathrm{TS}}^{o}$ increases towards 1 when $\rho \rightarrow 1$. However, $\lambda_{\mathrm{TS}}^{o}$ is not U-shaped; the reason is that the monopoly case is not well defined with CE demand: when $\rho \rightarrow 0$, the price $p$ tends to infinity, and therefore the output $q$ tends to zero.

Optimal degree of overlapping ownership ${ }^{24}$

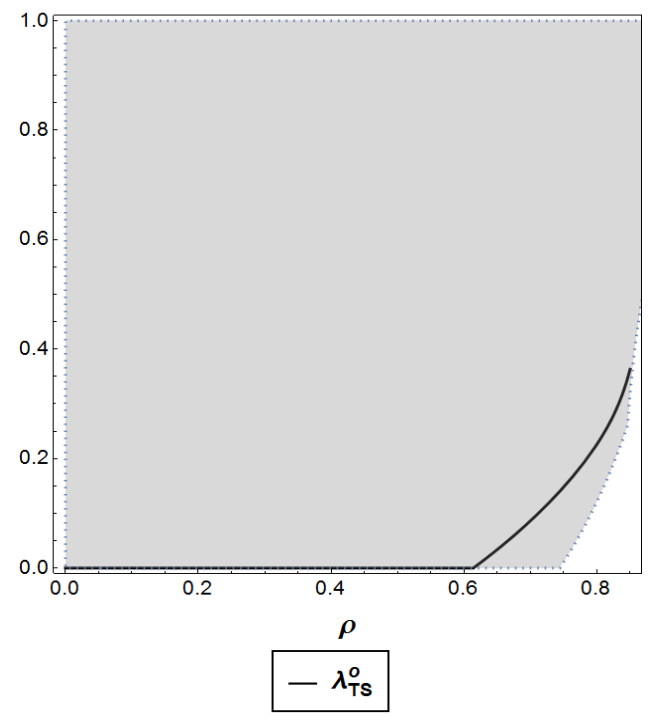

Fig. B15a. CE Bertrand model.

$$
(\alpha=0.5, \beta=0.25, n=6)
$$

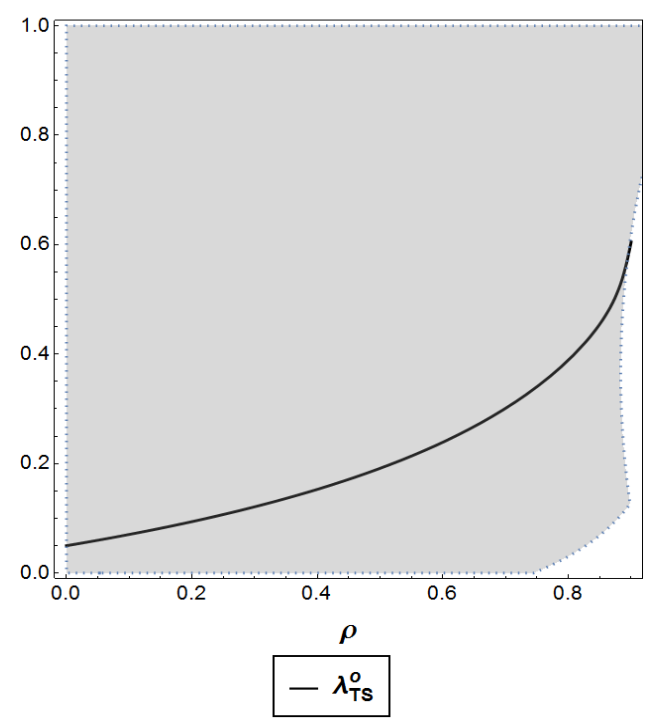

Fig. B15c. CE Bertrand model.

$$
(\alpha=0.5, \beta=0.75, n=6)
$$

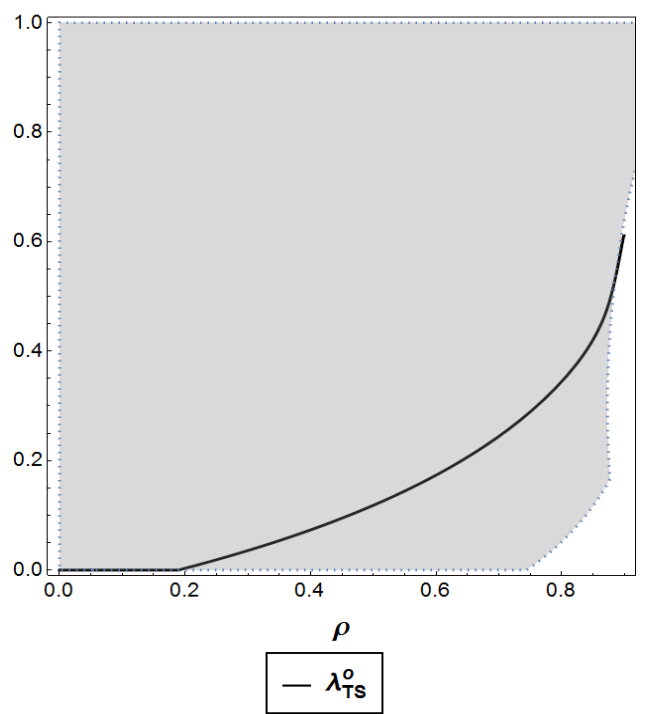

Fig. B15b. CE Bertrand model.

$$
(\alpha=0.5, \beta=0.5, n=6)
$$

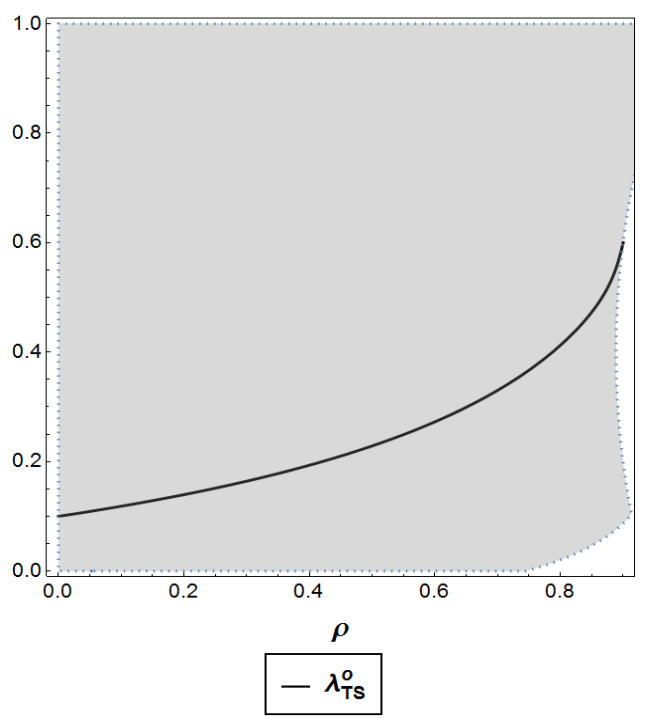

Fig. B15d. CE Bertrand model.

$$
(\alpha=0.5, \beta=1, n=6)
$$

${ }^{24}$ All simulations are conducted for $\kappa=1, Y=20$ and $\theta=0.05$. 
Two-stage model. Interior equilibrium. The interior equilibrium is characterized by the two FOCs (87) and (88), which at the symmetric equilibrium can be written as follows

$$
\begin{aligned}
& q^{*}+\left(p^{*}-c\left(B x^{*}\right)\right) v_{\lambda}=0 \\
& -c^{\prime}\left(B x^{*}\right) \tau q^{*}-1+\psi=0 .
\end{aligned}
$$

Next we derive the strategic effect, $\psi \equiv(n-1)\left(\partial \phi_{i} / \partial p_{j}\right)\left(\partial p_{j}^{*} / \partial x_{i}\right)$.

Strategic effect. The expression for $\partial \phi_{i} / \partial p_{j}$, which is strictly positive for $\lambda<1$, is given by (91). The expression for $\partial p_{j}^{*} / \partial x_{i}$ is computed in (82): $\partial p_{j}^{*} / \partial x_{i}=-\left(c^{\prime}(B x) / \Omega\right)(-\varphi)(\tilde{\beta}(\lambda)-\beta)$. By inserting equilibrium values into the definition of $\Omega$, given in equation (83), we get

$$
\Omega=\frac{\{(n-1)[(1+\mu) n+\lambda]+1\}(\alpha \tau S A / n)^{4 \alpha}(n-1) S^{2} A^{4}(1-\lambda)^{2}}{n^{4}[(n-1)(1-\lambda)+\mu n] \mu^{2} \kappa^{4}}>0 .
$$

The term $\varphi$ is defined in (85). By replacing $\partial_{p_{i} p_{i}} \phi_{i}$, given by (102), $\Delta_{p}$ given by (101), and $\partial D_{i}\left(\mathbf{p}^{*}\right) / \partial p_{i}$ and $\partial D_{k}\left(\mathbf{p}^{*}\right) / \partial p_{i}$ provided in Table B3, into $\varphi$ we obtain

$$
\varphi=-\frac{A^{4}\left(\frac{\alpha \tau S A}{n}\right)^{4 \alpha}(n-1)(1-\lambda)[n(1+\mu)+\lambda] S^{2}}{n^{4} \kappa^{4} \mu^{2}}<0 .
$$

To obtain $\tilde{\beta}(\lambda)$ we first have to calculate $\partial_{p_{i} p_{j}} \phi_{i}$, which using equation (92) and Tables B3 and B4 can be shown to be

$$
\partial_{p_{i} p_{j}} \phi_{i}=\frac{\Lambda(1-\lambda) S}{[(n-1)(1-\lambda)+n \mu] \mu p^{2} n^{2}} .
$$

As a result we have that

$$
\frac{\partial D_{i}\left(\mathbf{p}^{*}\right)}{\partial p_{i}} \partial_{p_{i} p_{j}} \phi_{i}-\lambda \frac{\partial D_{j}\left(\mathbf{p}^{*}\right)}{\partial p_{i}} \partial_{p_{i} p_{i}} \phi_{i}=-\frac{A^{4}\left(\frac{\alpha \tau S A}{n}\right)^{4 \alpha}(1-\lambda) S^{2}}{n^{4} \kappa^{4} \mu^{2}},
$$

which is strictly negative for $\lambda<1$. By inserting (107) and (108) into (84), and simplifying, we get

$$
\tilde{\beta}(\lambda)=-\frac{1}{(n-1)[(1+\mu) n+\lambda]}<0 .
$$

Consequently, the strategic effect is:

$$
\psi=-\frac{q^{*}}{v_{\lambda}}(n-1)(1-\lambda) \Lambda \frac{\partial D_{i}\left(\mathbf{p}^{*}\right)}{\partial p_{j}}\left[-\frac{c^{\prime}\left(B x^{*}\right)}{\Omega}(-\varphi)(\tilde{\beta}(\lambda)-\beta)\right]
$$

Let

$$
\omega(\lambda)=\frac{\frac{\partial D_{i}\left(\mathbf{p}^{*}\right)}{\partial p_{j}}(n-1)(1-\lambda) \Lambda \varphi}{\Omega v_{\lambda}}>0,
$$


then the strategic effect is shown to be negative:

$$
\psi=-c^{\prime}\left(B x^{*}\right) q^{*} \omega(\lambda)(\tilde{\beta}(\lambda)-\beta)<0 .
$$

